Project No. 10-921

\title{
Study of Interfacial Interactions Using Thin Film Surface Modification; Radiation and Oxidation Effects in Materials
}

\section{Fuel Cycle/Reactor Concepts RD\&D}

Dr. Kumar Sridharan University of Wisconsin-Madison

In collaboration with: Los Alamos National Laboratory

Sue Lesica, Federal POC Tedd Lister, Technical POC

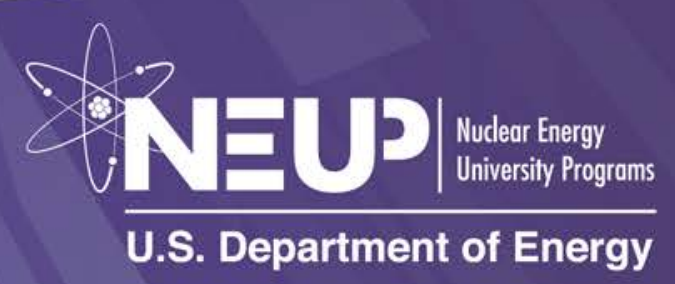




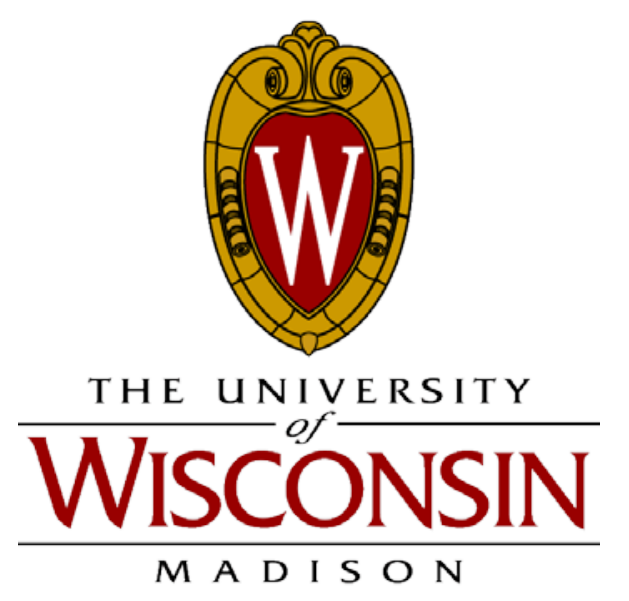

\section{Study of Interfacial Interactions using}

Thin Film Surface Modification: Radiation and Oxidation Effects in

\section{Materials}

Project Investigators: Dr. Kumar Sridharan, Dr. Todd Allen, Dr. Peng Xu, Dr. Clarissa Yablinsky (University of Wisconsin) and Dr. Jinsuo Zhang (Los Alamos National Laboratory)

Students: Mr. Alexander Mairov (graduate student) and Mr. Ben Hauch (research intern)

NEUP Final Report for Period: August 2010 to July 2013

Project Contact: Dr. Kumar Sridharan kumar@engr.wisc.edu

Date: January $8^{\text {th }}, 2014$ 


\section{Study of Interfacial Interactions using Thin Film Surface Modification: Radiation and Oxidation Effects in Materials}

Technical Work Scope Identification: MR-IIR (Mission Relevant - Investigator Initiated Research)

Proposal ID No.: 10-921

\section{Technical Abstract}

Interfaces play a key role in dictating the long-term stability of materials under the influence of radiation and high temperatures. For example, grain boundaries affect corrosion by way of providing kinetically favorable paths for elemental diffusion, but they can also act as sinks for both defects and helium generated during irradiation. Likewise, the retention of high temperature strength in nanostructured, oxide dispersion strengthened steels depends strongly on the stoichiometric and physical stability of the (Y, Ti)-oxide particle/matrix interface under radiation and high temperatures. An understanding of these interfacial effects at a fundamental level is important for the development of materials for use in the extreme environments of nuclear reactors.

The goal of this project is to evaluate the stability of interfaces by depositing thin films of materials on substrates, followed by ion irradiation of the film-substrate system at elevated temperatures and by post-irradiation oxidation treatments. Specifically, the research will be performed by depositing thin films of yttrium and titanium ( $500 \mathrm{~nm})$ on a Fe-12\% Cr binary alloy substrate. $Y$ and Ti have been selected as thin film materials because they form highly stable protective oxides layers. The $\mathrm{Fe}-12 \% \mathrm{Cr}$ binary alloy has been selected because it is representative of ferritic steels that are widely used in nuclear systems. The absence of other alloying elements in this binary alloy will allow for a clearer examination of structures and compositions that evolve during high temperature irradiations and oxidation treatments. The research is divided into four specific tasks: (1) sputter deposition of 500nm thick films of $Y$ and $\mathrm{Ti}$ on $\mathrm{Fe}-12 \% \mathrm{Cr}$ alloy substrates, (2) ion irradiation of the film-substrate system with $2 \mathrm{MeV}$ protons to a dose of $2 \mathrm{dpa}$ at temperatures of $300^{\circ} \mathrm{C}, 500^{\circ} \mathrm{C}$, and $700^{\circ} \mathrm{C}$, (3) oxidation of asdeposited and ion irradiated samples in controlled oxygen environment at $500^{\circ} \mathrm{C}$ and $700^{\circ} \mathrm{C}$, and (4) multi-scale computational modeling involving first-principle molecular dynamics (FPMD) and coarse grained dissipative particle dynamics (DPD) approaches to develop theories underlying the evolution and stability of structures and phases.

Samples from Tasks 1 to 3 (above) will be rigorously characterized and analyzed using scanning electron microscopy, Auger electron microscopy, x-ray diffraction, Rutherford back scatter spectroscopy, and transmission electron microscopy. Expected outcomes of the experimental work include a quantitative understanding of film-substrate interface mixing, evolution of defects and other phases at the interface, interaction of interfaces with defects, and the ability of the $\mathrm{Y}$ and Ti films to mitigate irradiation-assisted oxidation.

The aforementioned experimental work will be closely coupled with multi-scale molecular dynamics (MD) modeling to understand the reactions at the surface, the transport of oxidant through the thin film, and the stabilities of the deposited thin films under radiation and oxidation. Simulations of materials property changes under conditions of radiation and oxidation require multiple size domains and a different simulation scheme for each of these domains. This will be achieved by coupling first-principle molecular dynamics (FPMD) and coarse grained kinetic Monte Carlo (KMC). This will allow for a comparison of the results for each simulation approach with the experimental results. 


\section{TABLE OF CONTENTS}

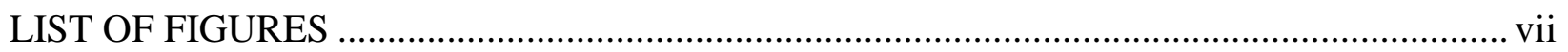

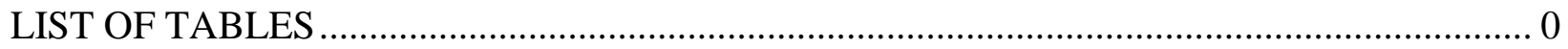

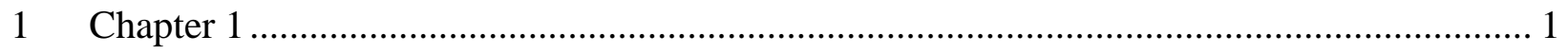

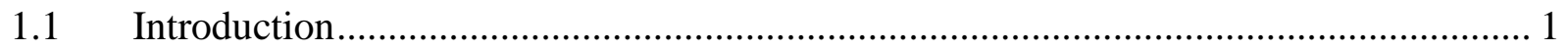

1.2 Interfaces in Nuclear systems …………………....................................................... 2

1.2.1 Interfaces in Nuclear Materials ............................................................................. 2

1.2.2 Radiation Damage............................................................................................. 2

1.2.3 Radiation Induced Phenomena ………………….................................................. 5

$1.3 \quad$ Ion Beam Processing …………………………................................................... 5

1.3.1 Ion Beam Mixing .................................................................................................. 6

1.3.1.1 Ballistic mixing....................................................................................... 7

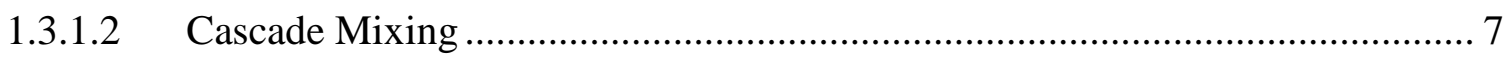

1.3.1.3 Thermodynamic effects in ions beam mixing ................................................. 10

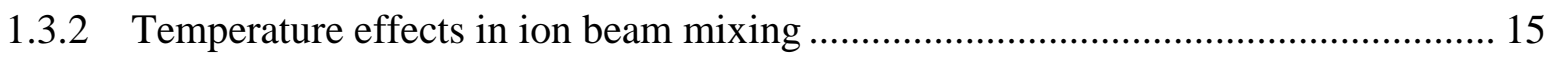

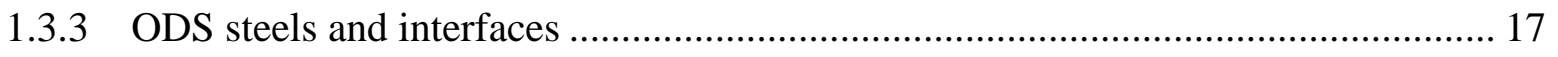

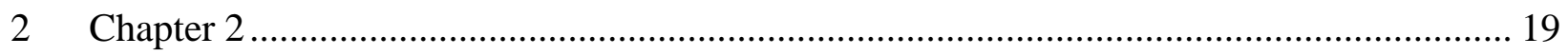

$2.1 \quad$ Multilayered systems ………………………….................................................. 19

2.1.1 Radiation damage effects in multilayered systems .................................................... 19

2.1.2 Radiation resistant multilayered systems ............................................................... 19

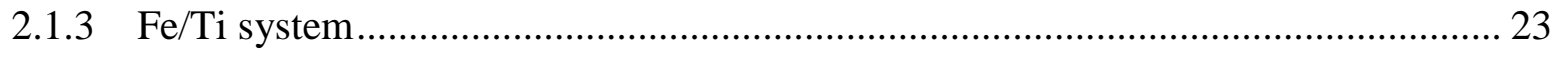

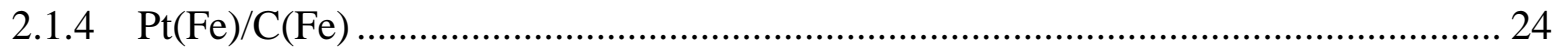




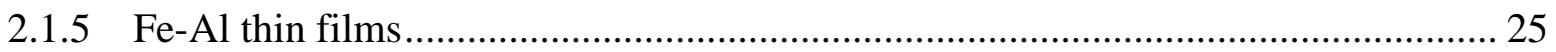

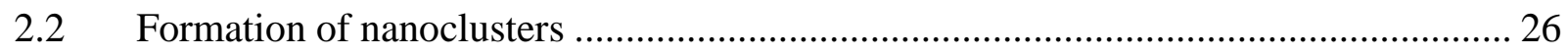

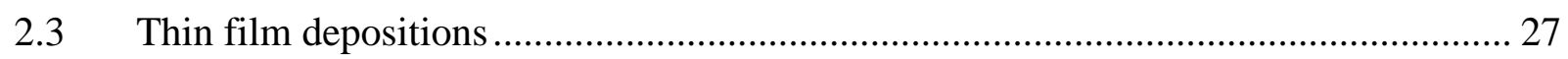

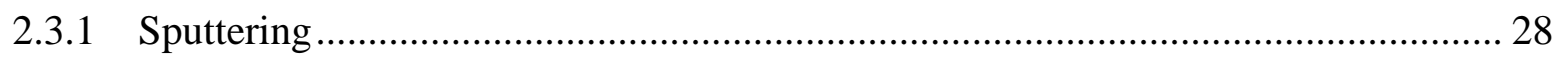

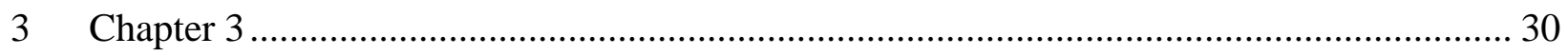

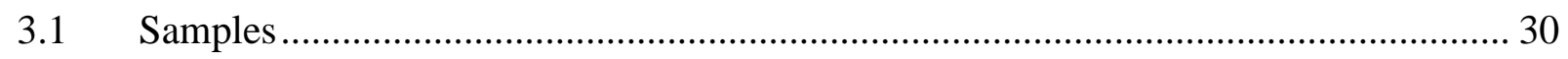

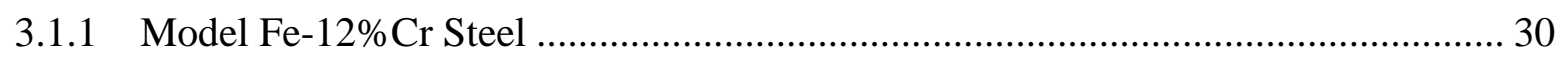

3.2 Thin film deposition of titanium and yttrium ............................................................. 32

3.3 Thin film deposition of nonconductive oxides ............................................................. 33

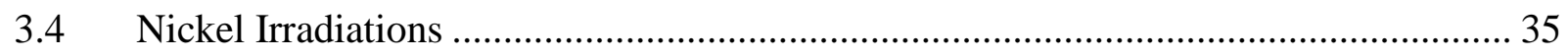

3.4.1 SRIM calculations and irradiation experiment design............................................... 35

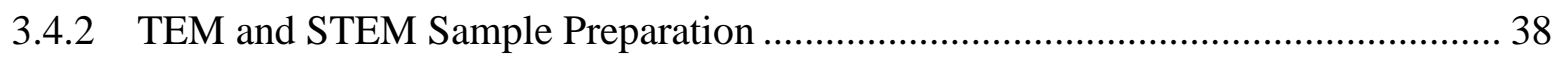

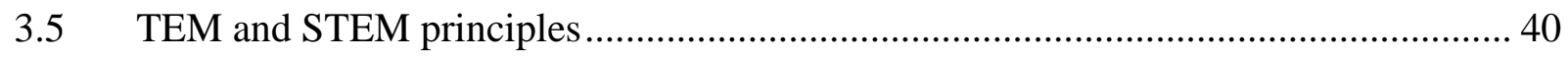

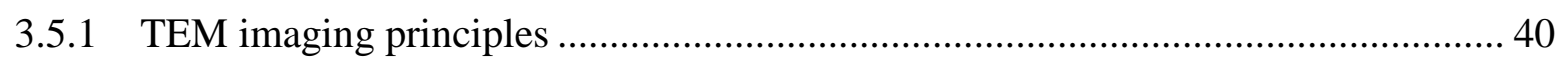

3.5.2 STEM imaging principles .................................................................................... 41

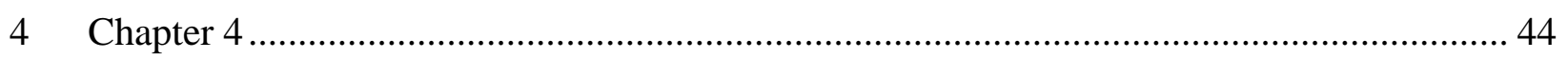

4.1 As-deposited metallic thin film examinations .................................................................... 44

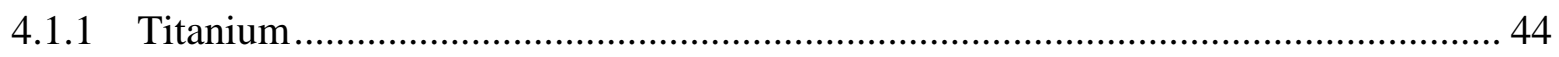

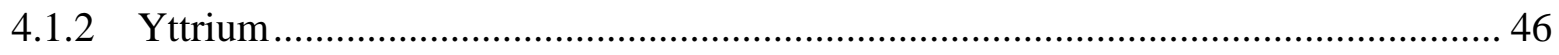

4.2 Irradiated \& Exposed metallic thin film examinations .................................................. 48

4.2.1 Titanium and yttrium-coated samples irradiated at $300^{\circ} \mathrm{C}$........................................ 48

4.2.1.1 Titanium-coated sample …………………….............................................. 48

4.2.1.2 Yttrium-coated sample ................................................................................ 49

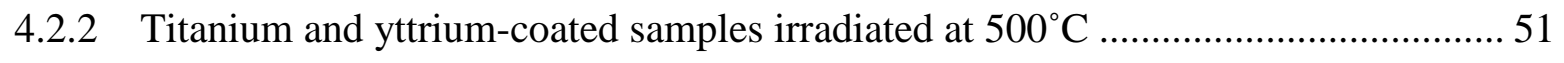

4.2.2.1 Titanium-coated samples ............................................................................... 51 


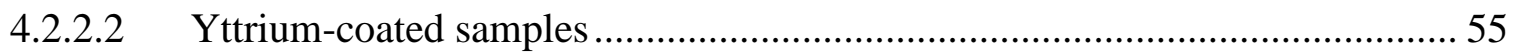

4.2.3 Titanium and yttrium-coated samples irradiated at $700^{\circ} \mathrm{C}$....................................... 57

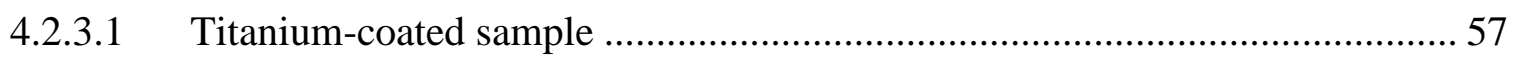

4.2.3.2 Yttrium-coated sample .................................................................................. 61

4.3 As-deposited oxide thin film examination................................................................. 62

4.4 Irradiated \& exposed oxide thin film examinations....................................................... 63

4.4.1 Titanium oxide and yttrium oxide-coated samples irradiated at $300^{\circ} \mathrm{C}$..................... 63

4.4.1.1 Titanium oxide-coated sample........................................................................ 63

4.4.1.2 Yttrium oxide-coated sample......................................................................... 64

4.4.2 Titanium oxide and yttrium oxide-coated samples irradiated at $500^{\circ} \mathrm{C}$....................... 67

4.4.2.1 Titanium oxide-coated sample...................................................................... 67

4.4.2.2 Yttrium oxide sample ……………………................................................... 69

4.4.3 Titanium oxide and yttrium oxide-coated samples irradiated at $700^{\circ} \mathrm{C}$..................... 70

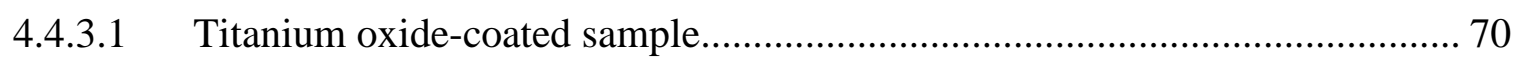

4.4.3.2 Yttrium oxide-coated sample......................................................................... 72

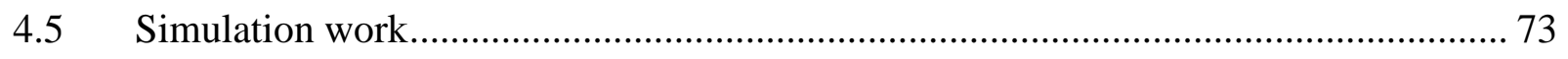

4.5.1 Irradiation damage studies on Fe-12\%Cr alloy ....................................................... 74

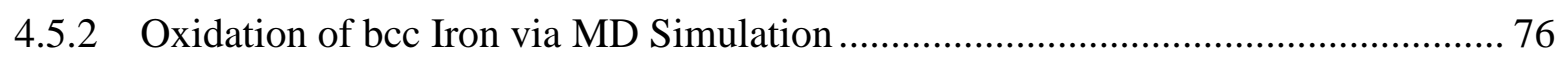

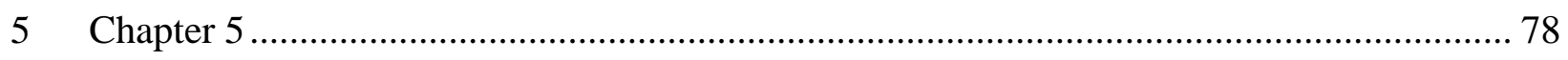

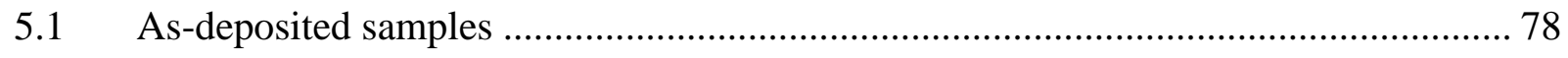

$5.2 \quad$ Radiation and temperature effects .......................................................................... 81

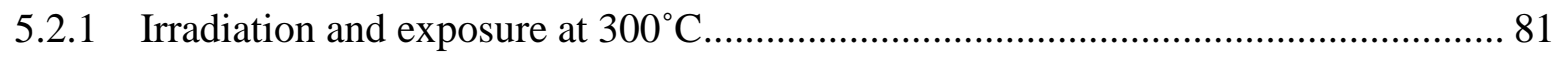

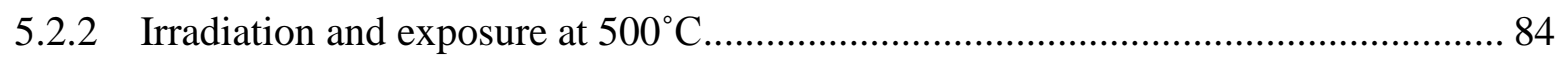

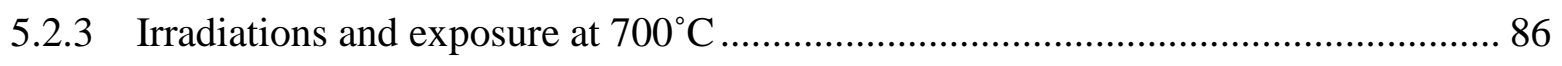

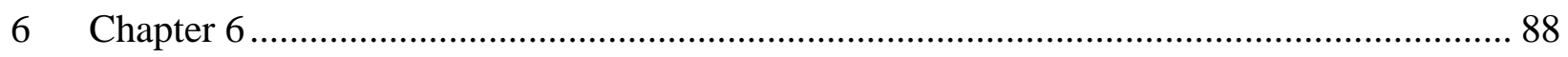




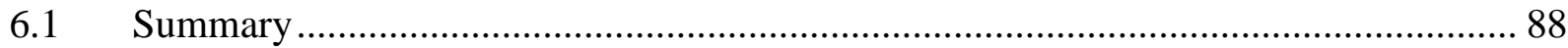

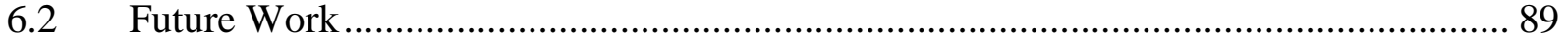

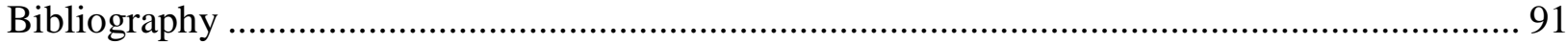




\section{LIST OF FIGURES}

Figure 1-1: Schematic process of a radiation damage event. A projectile particle hits atom of the target, which is displaced from its lattice position. As a result, a vacant and an interstitial site are formed (Frenkel pairs). In a real event, this process will go on several times before it will come to an end.

Figure 1-2: Radiation effects on material can cause a) void swelling, b) hardening and c) embrittlement (from (16) adapted from Ref. (17)).

Figure 1-3: Irradiation particle can cause atomic rearrangement of atoms across the interface in a bilayer sample causing atomic intermixing.

Figure 1-4: Backscattering signals obtained from the Sn marker embedded in amorphous Si (top) before irradiation (bottom) after $3 \times 10^{16}$ ions $/ \mathrm{cm}^{2} \mathrm{Ar}^{+}$irradiation (from Ref. (25))............. 8

Figure 1-5: The mixing parameter Dt $\phi$ plotted in logarithmic scale against $1 / 1000 \mathrm{~T}$. The filled circles are the result of (21) (after Ref. (25)).

Figure 1-6: $2 \mathrm{MeV}$ 24He + RBS-spectrum of a $\mathrm{Kr}^{+}$irradiated a) $\mathrm{W}$ deposited on the $\mathrm{Cu}<100>$ plane b) $\mathrm{Au}$ deposited on the $\mathrm{Cu}<110>$. Intense intermixing is observed in b), almost none is observed in a) (from Ref. (24)).

Figure 1-7: Enthalpy of mixing for an A-B compound. The broken line represents the enthalpy of bilayer system, while the continuous line represents the enthalpy of mixing of the compound A-B. If the enthalpy of mixing is negative, as in this case, forming an alloy is energetically more convenient than having a bilayer system (from Ref (20)).

Figure 1-8: Correlation between the mixing parameter and the heat of mixing for different bilayers irradiated with $600 \mathrm{KeV} \mathrm{Xe}{ }^{++}$at $77 \mathrm{~K}$ (from Ref (33))........................................... 13

Figure 1-9: Relationship ion mixing and cohesive energy for different bilayer systems.

Figure 1-10: Influence of temperature on ion beam mixing. Increasing the temperature above a certain value causes the amount of mixing to change rapidly with changes in temperature (from Ref. (37)).

Figure 1-11: Correlation between the average cohesive energy and the critical temperature (from Ref (38)).

Figure 1-12: Energy Filtered TEM images of a typical ODS steel. The black dots in the jump-ratio indicate regions depleted in iron, while the white spots in the Ti-L23 maps indicate regions enriched in titanium, i.e. the nanoclusters (from Ref. (49)) 
Figure 1-13: Schematic illustration of a) nanometer sized Y-Ti-O nanocluster embedded in a ferritic matrix and b) experimental analog involving high temperature ions irradiation of a $\mathrm{Y}_{2} \mathrm{O}_{3}$ thin film.

Figure 2-1: STEM images of the helium implanted $2.5 \mathrm{~nm}$ thickness $\mathrm{Cu} / \mathrm{V}$ multilayers. a) Surface region (no damage), b) At the damage peak region (200-325 nm) c) EDS chemical analysis. The individual layer morphology is maintained after helium implantation (from Ref. $(60))$.

Figure 2-2: Formation energies of point defects at the $\mathrm{Cu} / \mathrm{Nb}$ interface, compared to bulk $\mathrm{Cu}$ and $\mathrm{Nb}$. At the interface the formation energy is sensibly lower (from Ref (58)).....

Figure 2-3: XTEM micrographs of $\mathrm{Al} / \mathrm{Nb} 2.5$ multilayers after $100 \mathrm{KeV}$ helium implantation to a dose of $6 \times 10^{16}$ ions $/ \mathrm{cm}^{2}$ in the a) the surface region and b) the peak damage region: the individual layer structure is retained after irradiation (from Ref (64)).

Figure 2-4: Cross sectional high resolution TEM images of the Fe/Ti multilayers $19 \mathrm{~nm}$ thick submitted to thermal annealing at $450^{\circ} \mathrm{C}$ for $1 \mathrm{~h}$ (from Ref. (70)).

Figure 2-5: Cross sectional TEM images of the irradiated $\mathrm{Pt}(\mathrm{Fe}) / \mathrm{C}(\mathrm{Fe})$ samples a) $5 \times 10^{14}$ ions $/ \mathrm{cm}^{2}$ b) $1 \times 10^{15}$ ions $/ \mathrm{cm}^{2}$ c) HRTEM of a nanoparticle in b) (from Ref. (59; 75)).

Figure 2-6: CEMS analysis of the Fe-Al thin film bilayer furnace annealed in high vacuum (a and b), and $\mathrm{Ar}^{+}$and $\mathrm{Kr}^{+}$ion beam mixed (c and d).

Figure 2-7: Scheme for ion beam synthesis of nanostructures. When a material is implanted with high dose is supersaturated with impurity atoms is observed. The nanoclusters nucleate and grow during ion implantation if they have enough mobility, otherwise during subsequent

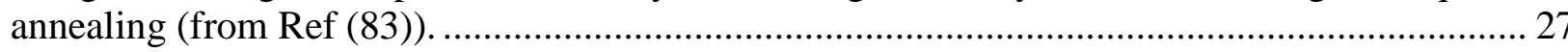

Figure 2-8: DC sputtering process: the target is negatively polarized with a DC voltage. The electrons in the system will be accelerated away from the target and will collide with the molecules of the gas filling the chamber. This interaction will ionize the gas molecules and the now positively charged particles will attract toward the cathode and the sputtering

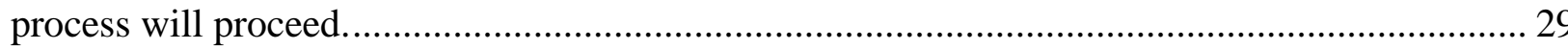

Figure 3-1: Fe-Cr binary phase diagram (from Ref (65))................................................ 31

Figure 3-2: View of the chamber of the Gentle Asher Chamber, Model A01, used to plasma clean the $\mathrm{Fe}-12 \% \mathrm{Cr}$ substrates before the deposition of the thin films.

Figure 3-3: Cross sectional SEM view of the titanium as-deposited sample. The titanium film was deposited on a Fe-12\% sample. After milling the sample to obtain a cross sectional view of the coating, the thickness was measured: by knowing the thickness displayed on the deposition system and comparing it with the actual thickness, the real deposition rate was determined. 
Figure 3-4: Denton evaporator (model DV-502) from the Material Science Center facility a) deposition chamber, power supply and deposition rate controller b) inside of the deposition chamber: quartz crystal thickness control, stage and shutter are visible.

Figure 3-5: $\mathrm{Y}_{2} \mathrm{O}_{3}$ sputter target a) front view b) side view with $\mathrm{Cu}$ backing plate. 34

Figure 3-6: WCAM Denton sputterer a) outer view of the deposition chamber b) inner view of the deposition chamber.

Figure 3-7: EMSL accelerator a) accelerator tank (3MV Tandem Van Der Graaff accelerator) b) set of quadrupole magnets in the high energy part of the beam.

Figure 3-8: Ti and Y samples after irradiation at $700^{\circ} \mathrm{C}$ to a dose of $8.74 \times 10^{6} \mathrm{ions} / \mathrm{cm}^{2}$. The arrows highlight black spots, corresponding to the region hit by the beam and the thermocouple, held on the surface of the sample by a clamp.

Figure 3-9: SRIM plots comparing the damage profile with the implanted Ni-atom profile for the $\mathrm{Y}_{2} \mathrm{O}_{3}$ coated samples. The energy of the ions was chosen to be $5 \mathrm{MeV}$ in order to minimize the concentration of the atoms implanted near the region of the interface.

Figure 3-10: Schematic of FIB lift out sample mounting and preparation technique a)flag mounting b)Chevron mounting....

Figure 3-11: SEM view of an electron-transparent yttrium as-deposited sample mounted in the Chevron position.

Figure 3-12: The two basic TEM operational principles a) diffraction mode, in which the diffraction pattern projects onto the viewing screen and b) image mode in which the actual image projects onto the screen.

Figure 3-13: Bright Field (BF) detector are axial detectors which collects the electrons that are transmitted right through the sample, Dark Field (DF) detectors collect those electron that are scattered at low angle and High Angle Annular Dark Field (HAADF) detectors collect those electrons that are scattered at high angle from the interaction with the sample.

Figure 4-1: BF TEM micrograph of a titanium sample in the as-deposited condition. A columnar structure is observed as is expected due to the use of the sputter deposition technique.

Figure 4-2: a) High Resolution TEM (HRTEM) micrograph along the [111] bcc zone axis of the titanium as-deposited sample, b) magnified substrate, and c) magnified interlayer. Regions in b) and c) have the same atomic structure.

Figure 4-3: Titanium as-deposited sample a) ADF micrograph, and b) the corresponding EDS line profile. A continually varying composition is seen in the interface.

Figure 4-4: BF TEM micrograph of the yttrium as-deposited sample.... 
Figure 4-5: Yttrium as-deposited sample a) ADF image, and b) corresponding EDS line profile. A much sharper interface is noted.

Figure 4-6: ADF images and EDS line scans of the titanium-coated sample ( $a$ and $b$ ) exposed at $300^{\circ} \mathrm{C}$, and (c and d) irradiated at $300^{\circ} \mathrm{C}$. As expected, the exposure for a short period of time $\left(210^{\prime}\right)$ at $300^{\circ} \mathrm{C}$ does not cause significant changes in either the morphology or the chemistry of the sample compared to the as-deposited case (Figure 4-3). Irradiation with nickel ions does broaden the interface.

Figure 4-7: ADF images and EDS line scans of the yttrium-coated sample (a and $b$ ) exposed at $300^{\circ} \mathrm{C}$, and (c and d) irradiated at $300^{\circ} \mathrm{C}$. Exposure for a short period of time at $300^{\circ} \mathrm{C}$ does not cause significant changes in the sample, while irradiation broadens the interface and helps form small precipitates.

Figure 4-8: ADF images and EDS line scans of the titanium-coated sample ( $\mathrm{a}$ and $\mathrm{b}$ ) exposed at $500^{\circ} \mathrm{C}$, and (c and d) irradiated at $500^{\circ} \mathrm{C}$. After exposure at $500^{\circ} \mathrm{C}$ for $210^{\prime}$ a wide intermixed region with intermediate composition forms between the substrate and the coating. Irradiation with nickel ions broadens this region. Titanium-enriched, iron-depleted nanoparticles are observed in both conditions.

Figure 4-9: a) ADF image of a titanium-enriched nanoprecipitate found along a grain boundary far from the original interface, b) enlargement of the same nanoprecipitate, and c) Composition profile along the nanoprecipitates. The chemical composition was obtained by running 5 different line scans separated by $1 \mathrm{~nm}$ and averaging the results.

Figure 4-10: ADF images and EDS line scans of three different nanoprecipitates found in the intermixed region of the titanium-coated sample exposed at $500^{\circ} \mathrm{C}$. The precipitates are spatially close to each other and their chemical composition is similar one to the other.

Figure 4-11: ADF images and EDS line scans of three different nanoprecipitates found in the intermixed region of the titanium-coated sample irradiated at $500^{\circ} \mathrm{C}$. The precipitates are spatially close to each other and their chemical composition is similar one to the other.

Figure 4-12: ADF images and EDS line scans of the yttrium-coated sample ( $a$ and $b$ ) exposed at $500^{\circ} \mathrm{C}$, and (c and d) irradiated at $500^{\circ} \mathrm{C}$. Exposure at $500^{\circ} \mathrm{C}$ for $210^{\prime}$ caused the formation of a wide intermixed region with almost constant composition. Irradiation causes the formation of regions more enriched in yttrium alternating to regions more enriched in iron. ..... 56

Figure 4-13: ADF images and EDS line scans of the yttrium rich inclusions found at the substrate-intermixed zone interface ( $a$ and b) exposed, (c and d) irradiated. In the circled insets of a) and c), the line scans alignment is shown.

Figure 4-14: Low-magnification ADF images of the titanium-coated sample do not show significant differences between exposed (a, b) and irradiated (c, d) conditions. 58

Figure 4-15: ADF images and EDS line scans of the titanium-coated sample. (a and b) exposed at $700^{\circ} \mathrm{C}$, and (c and d) irradiated at $700^{\circ} \mathrm{C}$. Near-coating nanoprecipitates are observed without the presence of an intermixed region. 
Figure 4-16: ADF images and EDS line scans of two different nanoprecipitates found in the titanium-coated sample irradiated at $700^{\circ} \mathrm{C}$. ( $\mathrm{a}$ and b) the precipitate close to the coating is enriched in titanium and depleted in iron, (c and d) the precipitate found along the grain boundary seems to be a metallic inclusion of titanium.

Figure 4-17: ADF images and EDS line scans of the yttrium-coated sample, (a and b) exposed at $700^{\circ} \mathrm{C}$, and $\left(\mathrm{c}\right.$ and d) irradiated at $700^{\circ} \mathrm{C}$. Irradiation effects appear to be masked by temperature-related effects.

Figure 4-18: ADF images and EDS line scans of three different nanoprecipitates found in the intermixing region of the yttrium-coated and $700^{\circ} \mathrm{C}$ irradiated sample. The three precipitates are found in the region enclosed in the red circle in a). Precipitates with a brighter contrast are of differing composition from the surrounding matrix. Similar results were obtained for the yttrium-coated, $700^{\circ} \mathrm{C}$ exposed sample without irradiation.

Figure 4-19: As-deposited ceramic thin film-coated samples (a and b) titanium oxide, (c and d) yttrium oxide. Yttrium is detected further into the substrate than titanium, while in each coated sample the oxygen has penetrated a comparable amount.

Figure 4-20: ADF images and EDS line scans of the titanium oxide-coated sample, (a and b) exposed at $300^{\circ} \mathrm{C}$, and (c and d) irradiated at $300^{\circ} \mathrm{C}$. Behavior is similar to the metal-coated samples: $300^{\circ} \mathrm{C}$ exposure for 3 hours does not significantly alter the sample from the asdeposited condition (Figure 4-19) while irradiation broadens the interface...

Figure 4-21: ADF images and EDS line scans of the yttrium oxide-coated sample, (a and b) exposed at $300^{\circ} \mathrm{C}$ and (c and d) irradiated at $300^{\circ} \mathrm{C}$. As opposed to the other thin film coated samples, the exposure for a few hours at $300^{\circ} \mathrm{C}$ does influence the sample morphology: chromium enrichment at the boundary is evident.

Figure 4-22: ADF images and EDS line scans of two nanoprecipitates buried in the Fe$12 \% \mathrm{Cr}$ substrate after irradiation at $300^{\circ} \mathrm{C}$ of the yttrium oxide-coated sample. The two precipitates are circled in red in a) and the corresponding EDS line scans are shown in c) and d). The two nanoparticles are enriched in both chromium and oxygen.

Figure 4-23: ADF images and EDS line scans of two different nanoprecipitates found in the intermixed region of the yttrium oxide-coated $\mathrm{Fe}-12 \% \mathrm{Cr}$ substrate after irradiation at $300^{\circ} \mathrm{C}$. The two precipitates circled in red in a) are magnified in b) and d), and the respective EDS line scans are shown in c) and e). The two particles exhibit similar iron enrichments.

Figure 4-24: ADF images and EDS line scans of the titanium oxide-coated sample (a and b) exposed at $500^{\circ} \mathrm{C}$ and (c and d) irradiated at $500^{\circ} \mathrm{C}$. Irradiation with nickel ions sputtered away part of the coating, and determined the formation of small $\mathrm{Cr}$ rich particles.

Figure 4-25: ADF images and EDS line of a nanoprecipitate found the titanium oxide-coated sample after irradiation at $500^{\circ} \mathrm{C}$. The precipitate circled in red in a) is shown magnified in inset b), along with an EDS line scan in c). The precipitate is enriched in chromium and depleted in titanium. 
Figure 4-26: ADF images and EDS line scans of the yttrium oxide-coated sample exposed at $500^{\circ} \mathrm{C}$ ( $\mathrm{a}$ and $\mathrm{b}$ ), and irradiated at $500^{\circ} \mathrm{C}\left(\mathrm{c}\right.$ and $\mathrm{d}$ ). The exposure at $500^{\circ} \mathrm{C}$ forms a nearinterface chromium-enriched region, followed by an iron-enriched region. The radiation field suppresses the formation of these layers, promoting the formation of a thicker layer with an altered chemical composition.

Figure 4-27: ADF images and EDS line scans of the titanium oxide-coated sample exposed at $700^{\circ} \mathrm{C}$ ( $\mathrm{a}$ and $\mathrm{b}$ ), and irradiated at $700^{\circ} \mathrm{C}$ (c and d). The exposure at $700^{\circ} \mathrm{C}$ does not cause broaden the substrate-coating interface despite the observed diffusion of $\mathrm{Cr}$ into the coating. Irradiation again sputters part of the coating, preventing the homogenous ingress of chromium.

Figure 4-28: ADF images and EDS line scans of the yttrium oxide-coated sample exposed at $700^{\circ} \mathrm{C}$ (a and b), and irradiated at $700^{\circ} \mathrm{C}\left(\mathrm{c}\right.$ and d). Exposure at $700^{\circ} \mathrm{C}$ forms large, Cr-rich nanoprecipitates close to the interface. Irradiation at the same temperature results in smaller near-interface nanoprecipitates that are now Y-rich.

Figure 4-29: ADF images and EDS line of a nanoprecipitate found the yttrium oxide-coated sample after irradiation at $700^{\circ} \mathrm{C}$. The precipitate circled in red in a) is shown magnified in b) along with an EDS line scan in c). The precipitate is enriched in chromium and depleted in titanium.

Figure 4-30. Final defect configuration after two PKAs were injected into the Fe-Cr binary alloy. Matrix atoms are not visualized.

Figure 4-31. (l) Graphical representation of the relationship between the number of Frenkel pairs and the PKA energy, as expressed by Equation 4-1. (r) The vacancy and interstitial configurations likely in an irradiated Fe-12\%Cr binary alloy. ....

Figure 4-32: $300^{\circ} \mathrm{C}$ iron surface oxidation simulation after (l) 67 and (r) 323 picoseconds of simulation using Aryanpour's Fe-O-C-H ReaxFF potential. Region A is thermally controlled, and the areas denoted by $\mathrm{B}$ are the oxygen introduction regions. Iron atoms are at the intersections of the black bonds, and oxygen atoms are shown as red spheres of arbitrary size. The simulation box extends vertically (not shown).

Figure 5-1: The relationship between the error function, erfx4Dt, and various relative magnitudes of the parameter $4 \mathrm{Dt}$.

Figure 5-2: Erf and Erfc fitting functions for the iron and titanium concentration profile of the titanium and yttrium as-deposited samples. The raw composition profiles are shown as dots.

Figure 5-3: Diffusion profile of iron and titanium in the titanium-coated sample exposed and irradiated at $300 \mathrm{C}^{\circ}$ : (a and c) experimental profiles (b and d) fitted profiles.

Figure 5-4: Damage profile for the titanium-coated sample irradiated with $5 \mathrm{MeV}$ nickel ions.. 82

Figure 5-5: Binary phase diagram of the Fe-Y system (from Ref (96)). 
Figure 5-6: Spherical precipitate with radius $r$ embedded in a matrix of thickness t. The acquisition of the nanoprecipitate's EDS spectrum will be influenced by the matrix................. 83

Figure 5-7: Binary phase diagram of the Fe-Ti system (from Ref. (65)). .................................. 84

Figure 5-8: $\mathrm{Fe}_{2} \mathrm{Ti}$ phase in the $\mathrm{Ti}$ coated sample a) irradiated at $500^{\circ} \mathrm{C} \mathrm{c}$ ) exposed at $500^{\circ} \mathrm{C}$. b) and d) are the SADP acquired from the dark grain in the center of the image and the red circles superimposed are the simulated diffraction pattern for the 1010 hcp zone axis.

Figure 5-9: Length-scale of interface mixing in function of temperature for the different samples exposed and irradiated at high temperature a) titanium b) yttrium............................. 87

Figure 6-1: Damage profiles of $5 \mathrm{MeV}$ nickel ions and $2.7 \mathrm{MeV}$ gold ions. ............................ 89 


\section{LIST OF TABLES}

Table 1: Composition of the Fe-Cr model alloy employed in this study (bal. Fe). The composition was obtained via ICP-OES

Table 2: Irradiation times (in minutes) for the samples irradiated in this work. The Ti- and the Y-coated samples were irradiated together at $700^{\circ} \mathrm{C}$; the oxidized samples were all irradiated two-by-two.

Table 3: The 4Dt parameter used to evaluate the sharpness of the interface in the asdeposited case.

Table 4: Interdiffusion coefficients of iron and titanium $\left(\mathrm{D}_{\mathrm{Fe}-\mathrm{Ti}}\right)$ and iron and yttrium $\left(\mathrm{D}_{\mathrm{Fe}-\mathrm{Y}}\right)$ as calculated from the fitting parameter of the concentration profile for the samples exposed and irradiated at $300^{\circ} \mathrm{C}$

Table 5: Enthalpy of mixing for different bilayers of interests for this work as calculated using the Miedema's model...... 


\section{CHAPTER 1}

\section{Introduction and Background}

\subsection{Introduction}

The United Nations predicts that the world population will grow from 6.6 billion in 2007 to 8.2 billion by 2030. This means a parallel increase in the energy demand, especially in developing countries, where, other than the population growth, many people are expected to see their living standards also increase (1). In fact, the UN Population Division predicts an ongoing and continuous trend of urbanization, reaching $70 \%$ worldwide by 2050 ; in that year, world population is predicted to be stabilized at about 9 billion with better food supply, sanitation, clean water, health, communication facilities and education (1). Instabilities in fossil fuel commodities and the production of greenhouse gases has led to a renewed push for energy technologies which have a stable market and a produce significantly lower carbon emissions. Nuclear energy is one of these technologies. In 2010, nuclear power generation provided some $12.8 \%$ of world electricity (compared to coal at $40.4 \%$, oil at $4.6 \%$, natural gas at $22.2 \%$, hydroelectricity at $16.3 \%$ and other sources at 3.6\%). Nuclear power generation is well-suited to the increasing demands because it is a reliable, large-scale, continuous source of electricity.

To meet demand for more energy, a road map for nuclear energy has been developed (2) and The Generation IV International Forum (GIF) was established to address key technical issues associated with designing, building and operating next-generation nuclear energy systems. Among the most promising goals of the Generation-IV designs are more efficient use of fuel, lower spent fuel waste production, economic competitiveness, and adherence to stringent standards of proliferation resistance and safety (3). Therefore, the advanced nuclear energy systems proposed under the Generation IV initiative are aimed at making revolutionary improvements in economics, safety and reliability, and sustainability.

The operating temperature is one of the most decisive factors in the economy of a nuclear reactor, because increasing the operating temperature allows for higher efficiency. Pressurized water-cooled reactors currently operate at about $315^{\circ} \mathrm{C}$, with pressure being the limiting factor towards operating at still higher temperatures (4). Generation IV systems anticipate operating at much higher temperatures, in higher radiation fields for and, some particular designs, also with more corrosive coolants than current light water reactors (5). A successful accomplishment of these goals requires, among other challenges, the development of a new class of materials which are able to maintain their chemical, physical, and mechanical, and dimensional stability under these extreme conditions. Of the candidate alloy systems being considered, ferritic-martensitic steels are expected to play an important role as cladding or structural components in Generation IV systems operating in the temperature range 350 to $700^{\circ} \mathrm{C}$ and to doses up to $200 \mathrm{dpa}$ (6). Ferritic Martensitic F/M steels, on account of their BCC structure show better swelling resistance than austenitic steels; however their use is limited $550-600{ }^{\circ} \mathrm{C}$ based on creep strength (7). To overcome this limitation, ODS steels have been developed. These steels are characterized by a fine dispersion of Y-Ti-O nanoparticles in the matrix of the steel. The presence of these 
nanoparticles can potentially increase the operation temperature up to about $650^{\circ} \mathrm{C}$ and thus increase the energy efficiency of Gen IV reactors.

In ODS steels and radiation resistant materials in general, the knowing the stability of the interfaces is increasingly important. Interfaces, in fact, play a key role in dictating the long-term stability of materials under the influence of radiation and high temperatures. For example, grain boundaries affect corrosion by way of providing kinetically favorable paths for elemental diffusion, but they can also act as sink for defects and helium generated during irradiation. Likewise, the stability of nanometer scale (Y, Ti)-oxide particles in the nano-structured oxide dispersion strengthened steels depends strongly on the stoichiometric and physical stability of the oxide particles/matrix interface under radiation and high temperatures (6). A fundamental understanding of these interfacial effects is crucially important to the development of materials for the extreme environments of Generation IV nuclear reactors. However, the interface between the nanoparticles (on the order of $\sim \mathrm{nm}$ ) (8) and the matrix is difficult to identify and characterize.

\subsection{Interfaces in Nuclear systems}

\subsubsection{Interfaces in Nuclear Materials}

An interface is defined as a surface forming a common boundary between adjacent regions, bodies, substances, or phases. In metals and alloys, typical internal interfaces are those between crystals of different orientation (grain boundaries, GB) and those of crystals of different structures (interphase interfaces). We have also interfaces when two solids are in intimate contact (thin film on a substrate) or simply when a surface is exposed to vacuum (solid-vacuum interface) (9). Interfaces are fundamentally important in determining materials' behavior and they have a technological importance in heterophase and homophase crystalline solids, beneficial in some cases and detrimental in others (10).

\subsubsection{Radiation Damage}

During service in a nuclear reactor system radiation-induced point defects are created in materials and can aggregate to form interstitial clusters, stacking faults, and voids (11). A radiation damage event can be defined as an interaction that causes the transfer of the kinetic energy of an incident ion and its redistribution to the atoms of a target. The radiation damage event is actually composed of many processes, as described in (12):

1. The interaction of the incident particle with a lattice atom.

2. The transfer of kinetic energy to the lattice atom resulting in the Primary Knock on Atom (PKA)

3. The displacement of the atom from the lattice site (if the energy is greater than the displacement energy ${ }^{1}$ )

4. The transfer of the PKA energy to other atoms of the lattice

5. The production of a displacement cascade

\footnotetext{
${ }^{1}$ The displacement energy is defined as the average energy necessary to displace an atom from its lattice position.
} 
As the PKA travels into the material it will interact usually with several other atoms, displacing them from their lattice position. A schematic and simplified example of this process is shown in Figure 1-1.

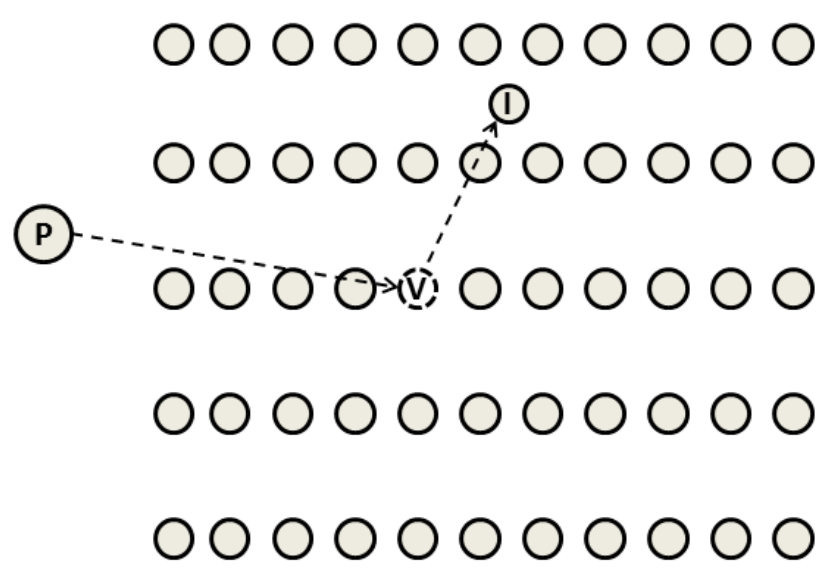

Figure 1-1: Schematic process of a radiation damage event. A projectile particle hits atom of the target, which is displaced from its lattice position. As a result, a vacant and an interstitial site are formed (Frenkel pairs). In a real event, this process will go on several times before it will come to an end.

These displaced atoms, in turn, will displace other atoms: if this sequence continues, we will have a collision cascade which is a "spatial cluster of lattice vacancies and atoms residing as interstitials in a localized region of the lattice" (12). The final results of the process described above, terminated usually when the PKA atoms comes at rest in the lattice, is the creation of a collection of point defects (interstitials and vacancies) which survives in the lattice.

To evaluate the response of a material to radiation damage, experiments in which specimen are irradiated in an experimental nuclear reactor can be undertaken; these experiments, though valuable, are time consuming, expensive, and they induce residual radioactivity in the irradiated samples. Most of these limitations can be overcome by the employment of charged particles. A characteristic advantage of charged-particle irradiation experiments is precise and individual control over most of the important irradiation conditions such as dose, dose rate, temperature, and quantity of gases present. Additional benefits are the absence of induced radioactivity of the specimens and, a greatly reduced irradiation time, which reduces from years to hours, to achieve comparable damage. Also, another fundamental application of this kind of experiment is the investigation of radiation effects in environments which are yet nonexistent, such as fusion reactors (13).

However, displacements caused from charged particles are different from those caused by neutrons: charged particles, while travelling into the material, lose energy not only through elastic collisions but also via electronic excitation. Radiation damage can be quantified solving the damage rate equation: 


$$
R_{D}=N \int_{\breve{E}}^{\hat{E}} \phi\left(E_{i}\right) \sigma_{D}\left(E_{i}\right) d E_{i}
$$

where $N$ is the lattice atom density, $\phi\left(E_{i}\right)$ is the energy dependent particle flux and $\sigma_{D}\left(E_{i}\right)$ is the energy dependent displacement cross section. The displacement cross section is the probability of the displacement of lattice atoms by incident particles and is defined as:

$$
\sigma_{D}\left(E_{i}\right)=N \int_{\breve{T}}^{\widehat{T}} \phi\left(E_{i}, T\right) v(T) d T
$$

Equation 1-2

where $\phi\left(E_{i}, T\right)$ is the probability that a particle of energy $E_{i}$ will impart a recoil energy $\mathrm{T}$ to a struck lattice atom, and $v(T)$ is the number of displaced atoms resulting from this type of collision (14). The result of radiation damage events can cause a significant rearrangement of atoms in the material. The energy transfer cross section, and hence the damage rate, is dependent on the type of particle and the incident particle's energy.

In the simplest approximation, (the hard-sphere approximation) the collisions are treated as purely ballistic; this approach, however, is suitable for neutron collisions. For ion-atom or atom-atom collisions, in fact, this model is invalid due to electron and nuclear interactions between ion-atom or atom-atom collision. To overcome this limitation, depending on the minimum separation distance between particles during a collision, a Coulomb, a screened Coulomb, or a Born-Mayer potential can be utilized. The result is that different ion types and energies lead to different cascade physics. Neutrons and heavy ions tend to produce large-scale and dense damage cascades that result in significant recombination during the cascade quench. Protons, on the other hand, tend to produce more localized and widely spaced cascades resulting in a higher number of freely migrating point defects.

A quantification of the damage caused by particle irradiation is needed in order evaluate the ability of a material to resist radiation. For charged particles species, the numerical values used to quantify the damage imparted to a sample can be derived from a freeware software based on Kinetic Monte Carlo simulations, known as Stopping Range of Ions in Matter (SRIM). SRIM calculates the stopping and range of ions (up to $2 \mathrm{GeV} / \mathrm{amu}$ ) into matter using a quantum mechanical treatment of ion-atom collisions. This calculation is made by the use of statistical algorithms which allow the ions to make jumps between calculated collisions and then averaging the collision results over the intervening gap; during the collisions, the ion and atom have a screened Coulomb collision, including exchange and correlation interactions between the overlapping electron shells.

The inputs that must be given to the program are the sample composition, the displacement energy of each of the elements of the system, the density of each layer, the incident ion type and its energy. The limitations of the program are absence of: time dependence, temperature dependence, and crystal structure. Moreover, only homogenous materials can be studied (other than layered compounds). Once a simulation is over, the damage rate output allows for a quantification of the damage caused by the irradiating process. The quantification is done in terms of the Displacement per Atom per second, dpa/s, which represents the number of times that each atom has been displaced per second, on average, from its lattice position and is defined as:

$$
\frac{d p a}{S}=\frac{\phi \cdot D}{N}
$$

Equation 1-3 
where $\phi$ is the particle flux, $D$ is the damage rate as given by SRIM and $\mathrm{N}$ is the atomic number density of the sample.

\subsubsection{Radiation Induced Phenomena}

The exposure of a material to a radiation field causes the formation of point defects at a concentration which is higher than the equilibrium one. Over time these defects migrate and aggregate to form extended defects, such as dislocation line or loops, vacancy loops, stacking fault, voids and bubble (15). The accumulation of these extended defects may change the properties of the original material through a series of different irradiation induced phenomena such as void swelling, irradiation growth, irradiation hardening, irradiation creep, and helium embrittlement. A generalized schematic of changes in material's properties due to extended defect formation from irradiation is shown in Figure 1-2.
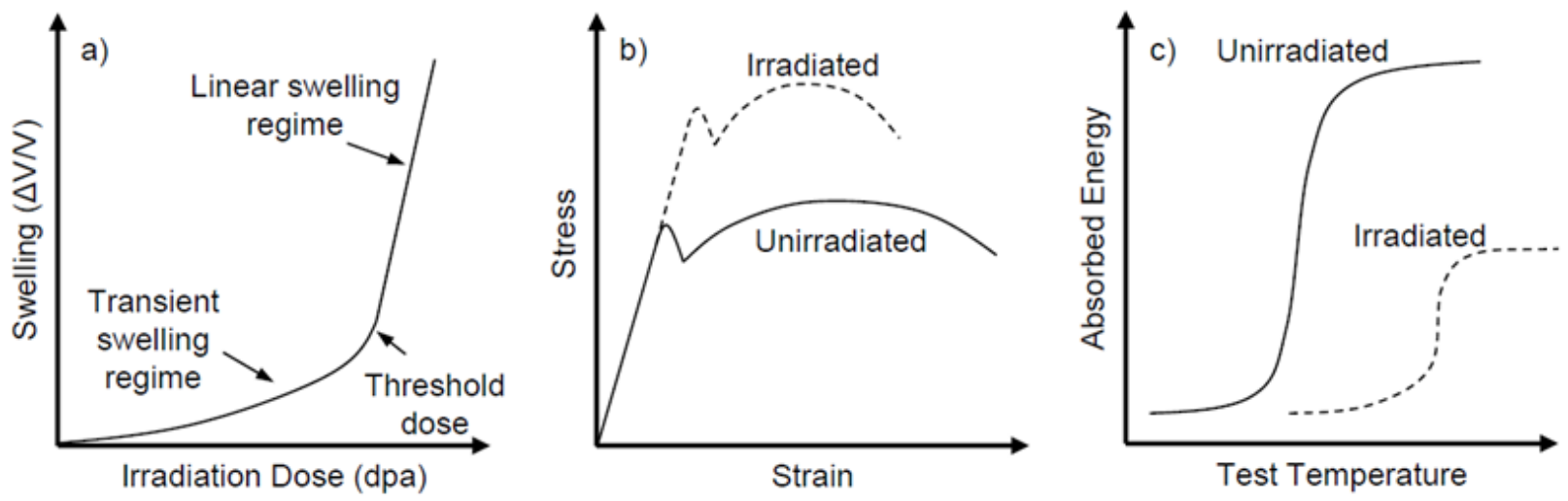

\section{Figure 1-2: Radiation effects on material can cause a) void swelling, b) hardening and c) embrittlement (from (16) adapted from Ref. (17)).}

\subsection{Ion Beam Processing}

Beams of energetic charged particles can also be used to modify and tailor the properties of materials and this has led to the field of ion beam processing in the last few decades. The first of these applications were based on ion implantation, in which an external species is implanted into a substrates by means of energetic ions: however, the very high doses required for typical application, like for example for increasing the hardness of steel or to form buried oxide layers in silicon, set a limit (represented by the sputtering yield ${ }^{2}$ ) to this technique (18).

To overcome those limitations, Ion Beam Mixing (IBM) was developed (19). IBM is defined as the characteristic effect of "atomic intermixing and alloying that can occur at the interface separating two different materials during ion irradiation” (20). In the following subsection, a brief overview about the IBM process will be given.

\footnotetext{
${ }^{2}$ The sputtering yield is defined as the average number of the atoms of the targets which are ejected for each incoming energetic ion.
} 


\subsubsection{Ion Beam Mixing}

When an energetic ion penetrates the solid, it slows down by the deposition of energy via interaction with the atoms and electrons of the target and ultimately comes to rest, if the target is thick enough (21). When these interactions occur with atoms of target which are found close to the interface between different layers, the processes of a radiation cascade can cause a significant redistribution across the sample, leading to the intermixing of the different layers composing the sample (Figure 1-3).

Schematically, different processes concur and must be taken into account to describe this physical phenomenon (20):

1. Ballistic mixing, in turn made up of

- Recoil Implantation

- Cascade Mixing

2. Thermodynamic effects

3. Temperature

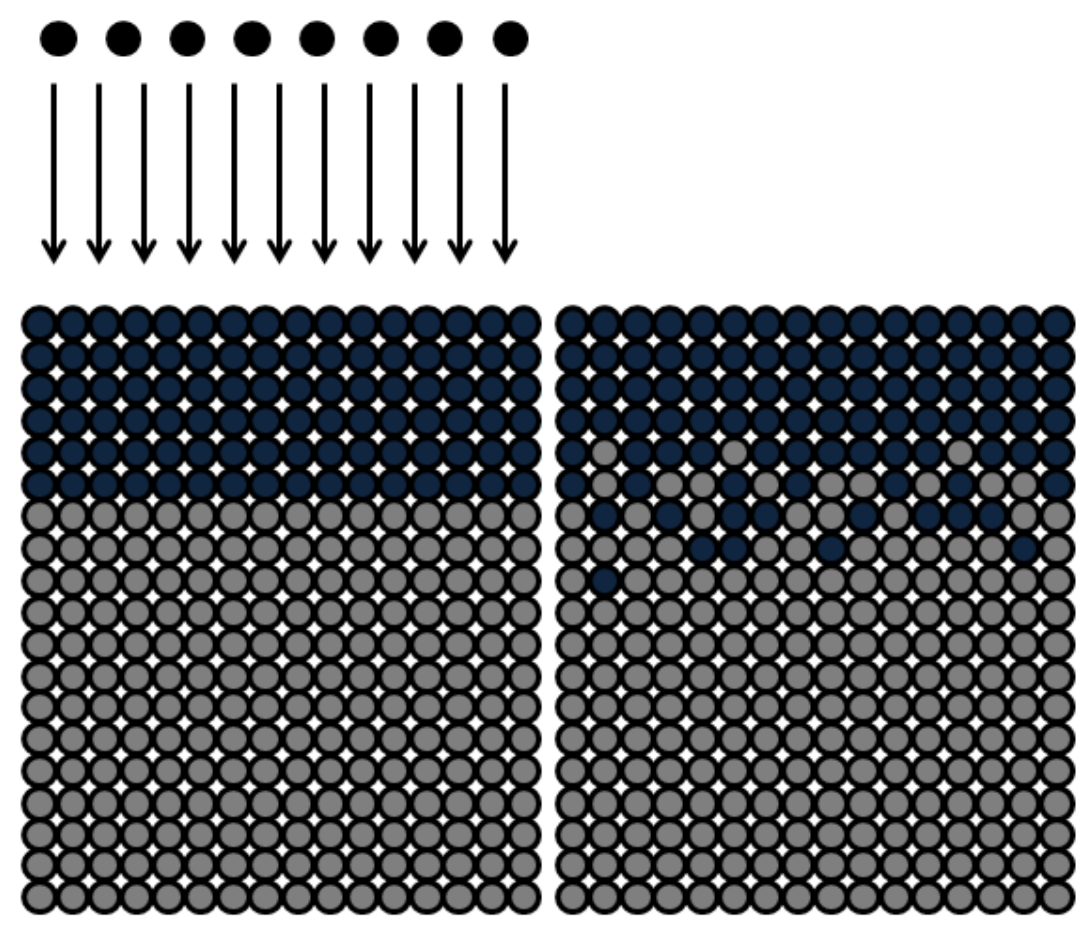

Figure 1-3: Irradiation particle can cause atomic rearrangement of atoms across the interface in a bilayer sample causing atomic intermixing. 


\subsubsection{Ballistic mixing}

The fundamental principle governing ballistic mixing is the displacement of atoms from their lattice sites and their relocation during IBM (20). In the classical scheme of a two body collision, an ion of mass $\mathrm{M}_{1}$ and energy $\mathrm{E}_{0}$ interacts with an atom from the substrate, with mass $\mathrm{M}_{2}$, supposed to be at the interface between the different layers. During subsequent collisions, the incoming ion loses part of its kinetic energy to the atoms of the target. The maximum amount of kinetic energy that can be loss in a single of these events is expressed by the following (22):

$$
T=\frac{4 M_{1} M_{2}}{\left(M_{1}+M_{2}\right)^{2}} E \cos ^{2}(\theta)
$$

Equation 1-4

Analysis of Equation 1-4 shows that the energy of the recoiling atom is dependent upon its mass, the energy of the incoming particle and the direction of the displacement. The maximum energy transfer is when the angle of incidence $\theta$ is zero (i.e., a head-on-collision). Analysis of the angle dependent scattering cross section shows however that low angle collision are far more probable and, therefore, in most of the collisions just a small fraction of the incident ion energy is transferred to the target (23). For example, analyzing the case of $100 \mathrm{KeV}$ argon ions incident on platinum, the number of platinum atoms recoiling at an angle $89^{\circ}$ is almost five orders of magnitude greater than the number of platinum atoms recoiling at $0^{\circ}$, the case of the head-on collision (23). The process just described, is the simplest form of ballistic mixing and is known as recoil implantation or recoil mixing (20). Describing IBM phenomena as made up of single events, in which atoms of the target are displaced from their lattice positions, is not the most exhaustive way to treat the problem. In fact, an energetic ion will be responsible for the displacement of several atoms during its path into the solid and those displaced atoms will in turn displace several other atoms on their own, before they will lose all the energy they gained in their first collision, coming to rest in the matrix.

\subsubsection{Cascade Mixing}

Cascade mixing is a far more efficient mixing mechanism than recoil implantation, because the several displacements occurs as a result of impacts with recoiling matrix atoms, which are not only much more numerous than direct impacts with the incident ions, but they also take place at lower energies and consequently have a larger cross-section (24). To have a better understanding of these collisional effects, marker studies, consisting of the ion bombardment of a thin layer of markers inserted between two layers of the matrix have provided new insights into the process (25).

In the work of Paine at al. (25), three different systems were studied: Ge and Sn markers embedded in amorphous $\mathrm{Si}$, and a Si layer buried in a Ni sample. The systems were bombarded with argon ions to fluences between $3 \times 10^{15}$ and of $3 \times 10^{16}$ ions $/ \mathrm{cm}^{2}$ at different temperatures $(40 \div 300 \mathrm{~K})$. Figure 1-4, taken the work of Paine et al, shows the isotropic spreading of the backscattering signals from the $\mathrm{Sn}$ after irradiation. 


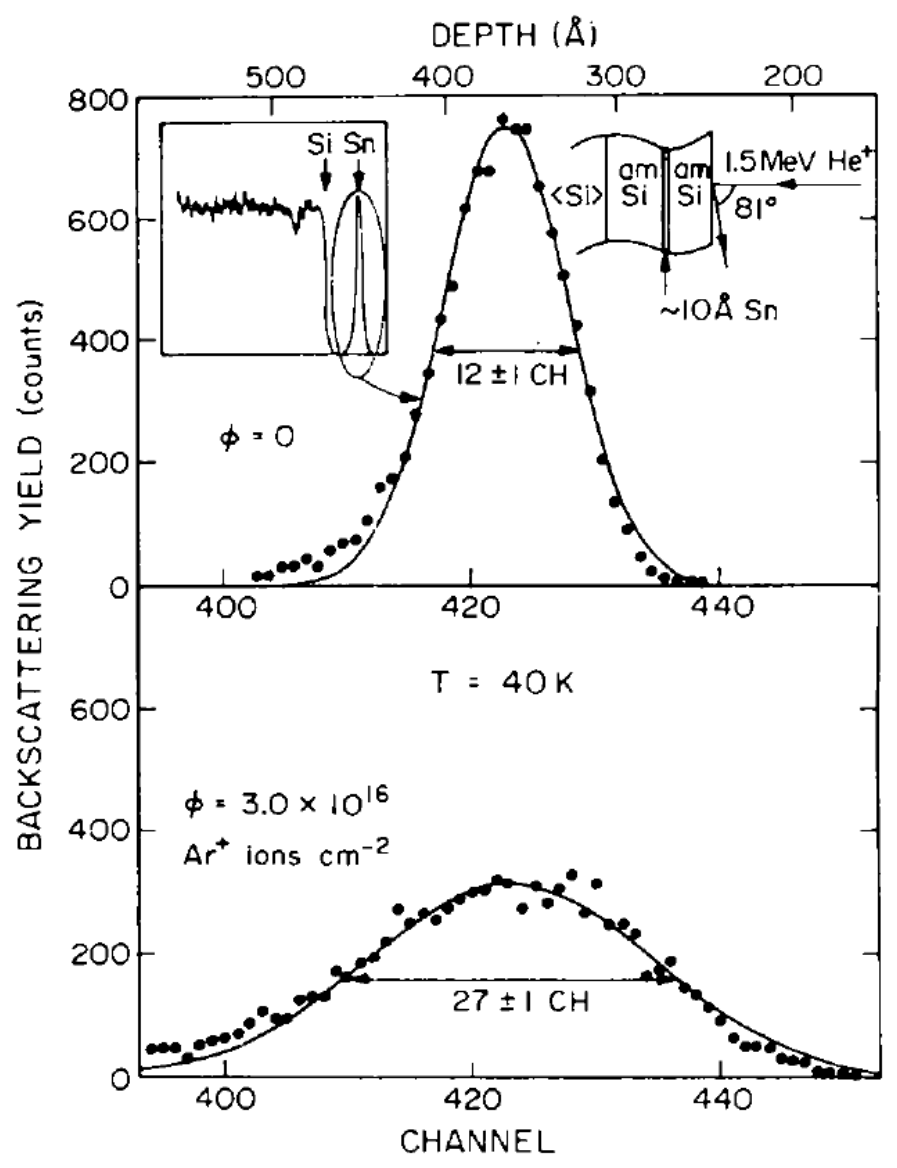

Figure 1-4: Backscattering signals obtained from the Sn marker embedded in amorphous Si (top) before irradiation (bottom) after $3 \times 10^{16}$ ions $/ \mathrm{cm}^{2} \mathrm{Ar}^{+}$irradiation (from Ref. (25)).

The spreading of the Gaussian distribution is a clear indication that the process of displacement occurs in a symmetrical fashion, and can be quantified as follows (25):

$$
\Omega^{2}=\Omega_{p o s t}^{2}-\Omega_{p r e}^{2}
$$

Equation 1-5

where $\Omega_{\text {post }}^{2}$ and $\Omega_{\text {pre }}^{2}$ are the variances of the curves fitted to the marker profile before and after the irradiation. The characteristic profile of Figure 1-4 suggests contribution from diffusion which can be expressed as follows (26):

$$
V_{i r r}^{2}=2 D t
$$

Equation 1-6

where $\mathrm{V}$ is the variance in units of distance and it's related to the experimentally measured variance via the following relation:

$$
V=\frac{\Omega}{S_{a}^{B}}
$$


where $S_{a}{ }^{B}[S]$ is the RBS energy loss conversion factor for a marker of atoms $A$ in a matrix of atoms B (20). These results have been analyzed also in terms of a quantity called mixing parameter $D t / \phi(21)$, where $\mathrm{D}$ an effective diffusion coefficient, $\mathrm{t}$ is the duration of the cascade and $\phi$ is the dose of irradiation (the value of $D t$ can be deduced from $\Omega^{2}$ ). The work of Paine et al. (25) indicates two important things about the mixing parameter:

1. Within the uncertainty of the experiments $D t / \phi$ is constant for $\mathrm{Ar}^{+}$irradiation in the temperature range (40K-300K)

2. Ion beam mixing experiments up to $\sim 300 \mathrm{~K}$ occurs by collision cascades in these systems

The latter point was proven by fitting an Arrhenius relation, $\exp \left(-E_{a c t} / K T\right)$, to their data, which gives a maximum possible value of the thermal activation energy of $4 \times 10^{-3} \mathrm{eV}$. This value has to be compared with activation energies of around $1 \mathrm{eV}(27)$ that might are to be expected for migration of vacancies in the solids they studied. This confirms that ion beam mixing experiments up to $\sim 300 \mathrm{~K}$ occurs by collision cascades.

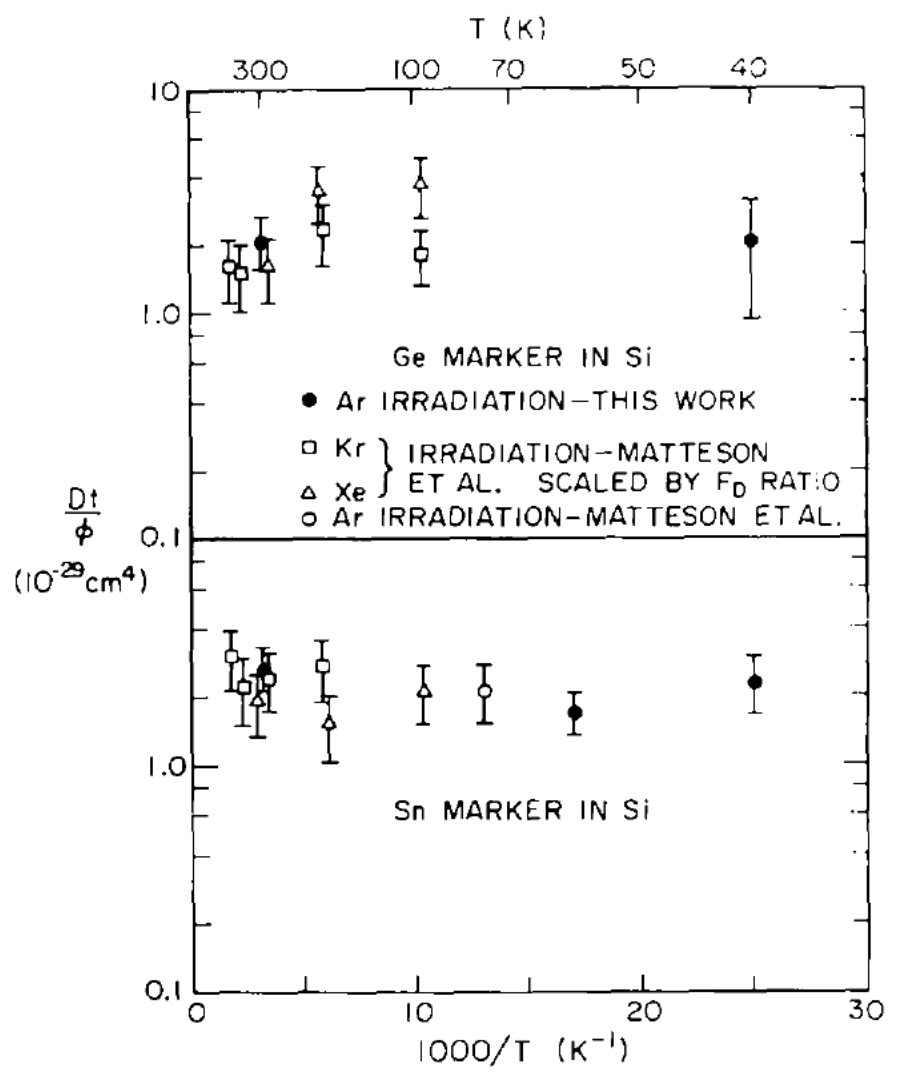

Figure 1-5: The mixing parameter $D t / \phi$ plotted in logarithmic scale against 1/1000 T. The filled circles are the result of (21) (after Ref. (25)). 
Other works show similar results. Figure 1-5 shows the results for the mixing parameter $D t / \phi$ as a function of temperature $\mathrm{Ar}^{+}, \mathrm{Kr}^{+}$and $\mathrm{Xe}^{+}$irradiations of amorphous different elements (Ni, Ge, Sn, Sb, Pt and Au) buried into Si samples, deduced from the results of Matteson et al. (21) and there is quantitative agreement between the results of Paine et al. (25) and those of Matteson et al. (21) at the higher temperatures where the two investigations overlap.

To develop a mathematical formulation of the mixing parameter, the findings that most recoils are produced near the minimum energy necessary to displace atoms (23), with the initial momentum of the incident particle which is soon lost and the overall momentum of the cascade becomes isotropic, are used. Borrowing from the random walk diffusion theory, the effective diffusivity can be written as (28):

$$
D_{\text {cas }} t=\frac{n_{d}\left\langle r^{2}\right\rangle}{6}
$$

where $n_{d}$ is the number of cascade induced displacement per atom (dpa), $\left\langle r^{2}\right\rangle$ is the mean squared range of the displaced atom and $t$ is the cascade lifetime. The dpa can be expressed as:

$$
n_{d}=\frac{0.4 F_{d} \phi}{E_{d} N_{0}}
$$

where $F_{d}$ is the damage energy per unit of length, $\phi$ is the ion dose and $N_{0}$ is the atomic density. Combining equations

and

$$
D_{\text {cas }} t=0.067 \frac{F_{d}\left\langle r^{2}\right\rangle}{N_{0} E_{d}} \phi
$$

More detailed calculation can be found in literature; they take into account the mass difference between the ion and the target atoms, and they are based on transport theories (29). Error! Reference source not found. shows that the effective diffusion coefficient is linear in both the dose and the damage energy and this has been confirmed by experiments (21).

\subsubsection{Thermodynamic effects in ions beam mixing}

To introduce the importance of thermodynamic effects in ion beam mixing, let's start with an example taken from literature, by comparing two different bilayer samples which has a similar ballistic behavior, but that show a different damage evolution when irradiated: $\mathrm{Au}$ on $\mathrm{Cu}$ and $\mathrm{W}$ on $\mathrm{Cu}$. Considering the parameters of importance for ion-solid interactions (atomic density, atomic number and atomic masses) these two systems should behave similarly when irradiated at similar conditions. RBS data, show that this is not the case (24), see Figure 1-6. 
a)

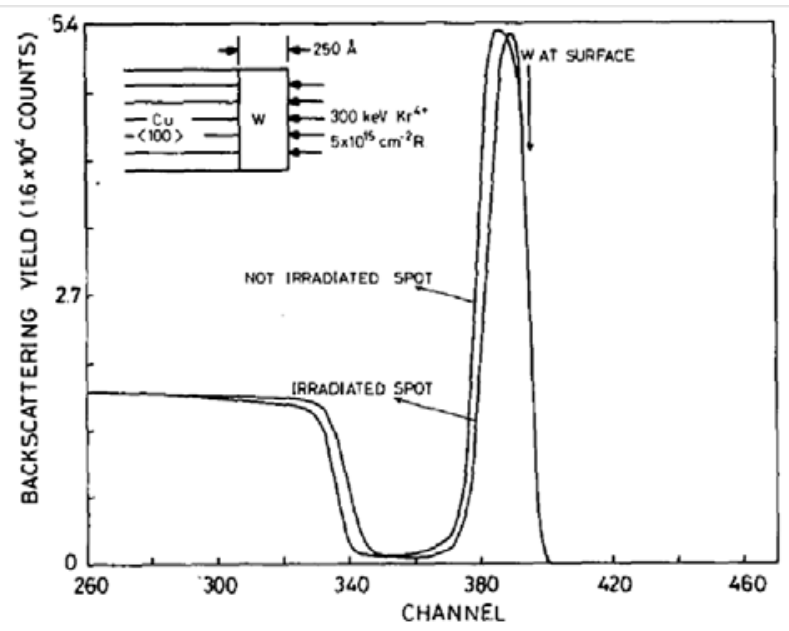

b)

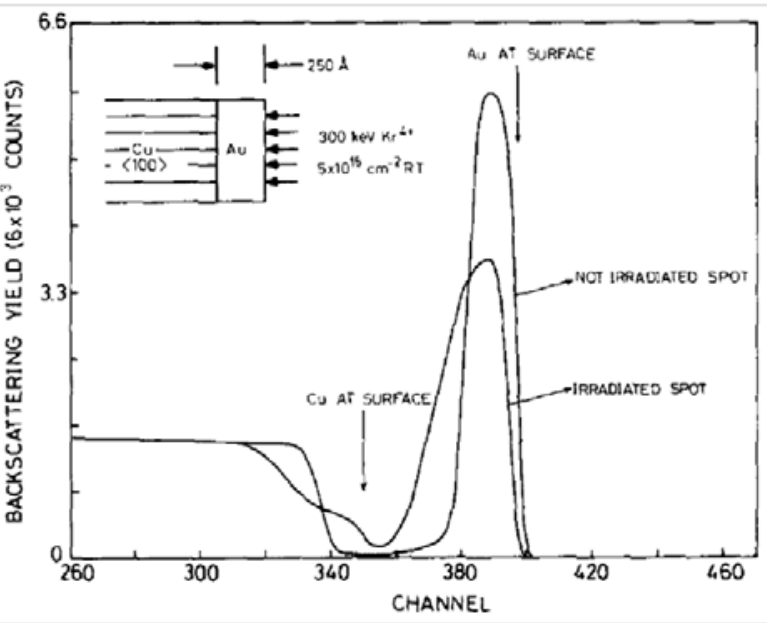

Figure 1-6: $2 \mathrm{MeV}_{2}^{4} \mathrm{He}^{+} \mathrm{RBS}$-spectrum of a $\mathrm{Kr}^{+}$irradiated a) $\mathrm{W}$ deposited on the $\mathrm{Cu}<100>$ plane b) $\mathrm{Au}$ deposited on the $\mathrm{Cu}<110>$. Intense intermixing is observed in b), almost none is observed in a) (from Ref. (24)).

The fact that systems which have similar ballistic parameters can behave differently under irradiations suggests that there are also other parameters which should be taken into account. Other systems studied in literature like Hf-Ni and Hf-Ti (30), suggest that this parameter is the enthalpy of mixing. The enthalpy of mixing is an indicator of the attraction between the atoms of the different elements relative to the attraction between atoms of the same element. When the enthalpy of mixing is positive, mixing is endothermic and when the enthalpy of mixing is negative, mixing is exothermic. According to regular solutions theory the enthalpy of mixing can be written as (31):

$$
\Delta H_{\text {mix }}=X_{A} X_{B} \Omega_{H}
$$

Equation 1-11

where $X_{A}$ and $X_{B}$ are the molar fraction of the elements A and B and $\Omega_{H}$ can be approximated with:

$$
\Omega_{H} \sim Z_{c} N_{a}\left[H_{A B}-1 / 2\left(H_{A A}+H_{B B}\right)\right]
$$

Equation 1-12

where $Z_{c}$ is the coordination number of the lattice cell, $N_{a}$ is the Avogadro's number and $H_{i j}$ is the mean potential between atom pairs. In layered system with negative enthalpy of mixing, intermixing is favored by this thermodynamic driving force and, on the other hand for systems with positive heat of mixing, the de-mixing becomes the driving force and it can overwhelm ballistic effects occurring during irradiation of the system, especially at high temperatures (32). The change in enthalpy that occurs when intermixing two layers, A and B during ion mixing is schematically shown in Figure 1-7. 


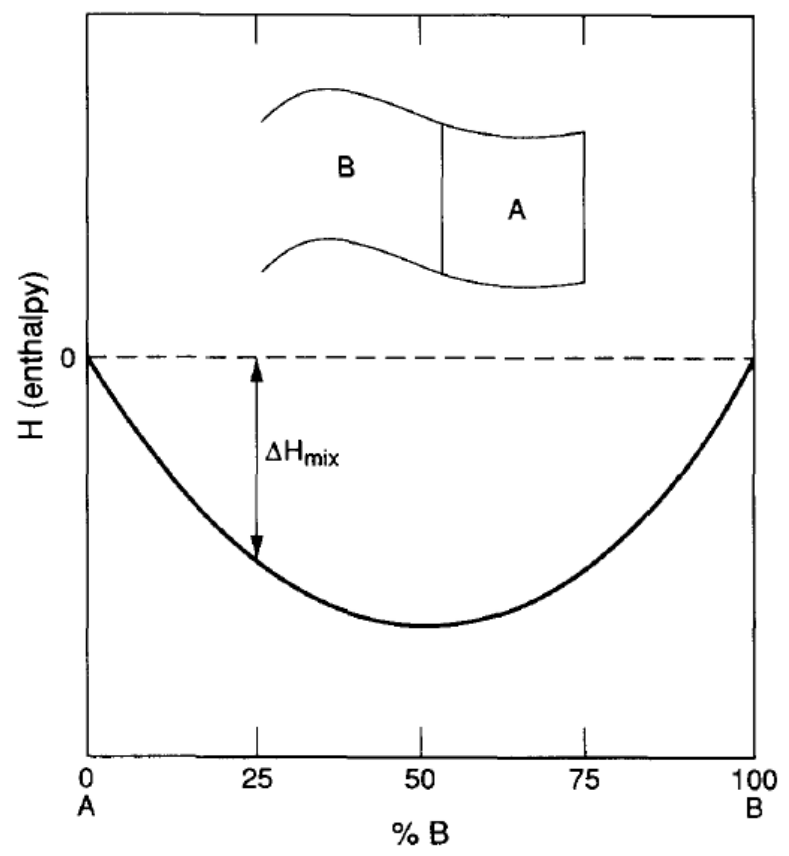

Figure 1-7: Enthalpy of mixing for an A-B compound. The broken line represents the enthalpy of bilayer system, while the continuous line represents the enthalpy of mixing of the compound $A-B$. If the enthalpy of mixing is negative, as in this case, forming an alloy is energetically more convenient than having a bilayer system (from Ref (20)).

Thermodynamic effects are not pointed out by marker experiments because, as can be seen from Figure 1-7, in those studies the concentration of one alloying element is too low to have appreciative thermodynamic effects, as can be also seen in bilayers with similar ballistic properties but different enthalpies of mixing (Pt or Au with a $3 d$ metal as $\mathrm{Ti}, \mathrm{V}, \mathrm{Cr}, \mathrm{Mn}, \mathrm{Co}, \mathrm{Ni}$ ). To avoid effects related to temperature such as radiation enhanced diffusion, their experiments were performed at $\mathrm{LN}_{2}$ temperature. The irradiations were performed with $\mathrm{Xe}^{++}$ions at $600 \mathrm{KeV}$ and doses between $10^{15}$ and $5 \times 10^{15}$ ions $/ \mathrm{cm}^{2}$. The results are shown in Figure 1-8. 


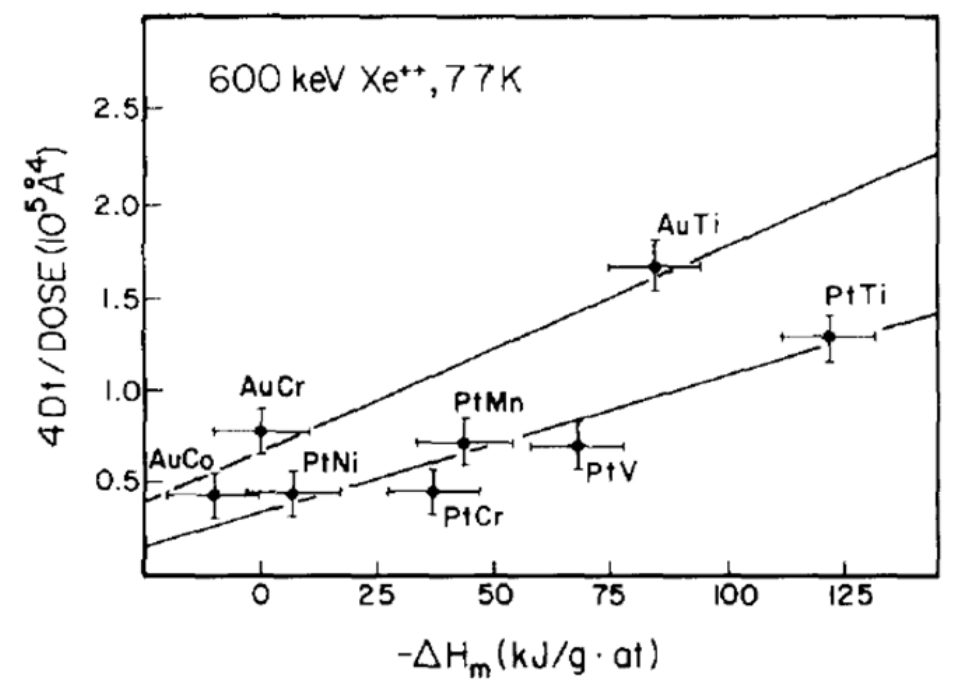

\section{Figure 1-8: Correlation between the mixing parameter and the heat of mixing for different bilayers irradiated with $600 \mathrm{KeV} \mathrm{Xe}{ }^{++}$at $77 \mathrm{~K}$ (from Ref (33)).}

As can be seen from Figure 1-8 there is a linear relationship between the mixing parameter and the heat of mixing for the different bilayer samples analyzed. The most effective way to take into account these observations is to use the approach developed by Darken, who developed an expression for diffusion in presence of a chemical driving force (26). Following his approach the interdiffusion coefficient can be written as:

$$
\widetilde{D}=\left(D_{1}^{*} X_{2} D_{2}^{*} X_{1}\right)\left(1+\frac{d \ln \gamma_{1}}{d \ln X_{1}}\right)
$$

where $D_{i}^{*}, X_{2}$ and $\gamma_{i}$ are the diffusion coefficient, the mole fraction and the activity coefficient of the element $i$. For the determination of the diffusion coefficient, is possible to express the mixing rate equation as:

$$
\widetilde{D}=\widetilde{D}_{0}\left(1-\frac{2 \Delta H_{m i x}}{K_{B} T}\right)
$$

where $\widetilde{D}_{0}$ can be taken from Error! Reference source not found..

The heat of mixing however is not able alone to take into account the rate of mixing observed experimentally when intermixing some metallic bilayers (34). Systems which have similar ballistic properties behave differently when intermixed even for $\Delta H_{\text {mix }}=0$. There is another thermodynamic factor that must be taken into account to evaluate intermixing effects in bilayers and that is the cohesive energy. The cohesive energy is defined as the difference of energy of a solid as compared to the energy of the same material when all the atoms have been brought to infinite distance. Generally, materials with high cohesive energy have high melting points and vice versa (20). Therefore, is reasonable that materials with high cohesive energy, the cascade induced by the ion bombardment will be quenched sooner than materials with a low 
cohesive energy, leading to the conclusion that a low cohesive energy will favor intermixing. Experimentally, is has been found that:

$$
E_{V}^{F} \sim-0.29 \Delta H_{c o h}
$$

Defining the activation energy for self-diffusion $\left(\mathrm{E}_{S D}\right)$ as:

$$
\mathrm{E}_{S D}=E_{V}^{F}+E_{V}^{M}
$$

where $E_{V}^{F}$ and $E_{M}^{F}$ are the activation energy for the vacancy formation and migration and using the relationship developed in (35):

$$
E_{V}^{F} \sim 0.55 \mathrm{E}_{S D}
$$

It is then possible to write:

$$
\mathrm{E}_{S D} \sim 0.53 \Delta H_{c o h}
$$

Figure 1-9 shows the correlation between the mixing parameter and the cohesive energy.

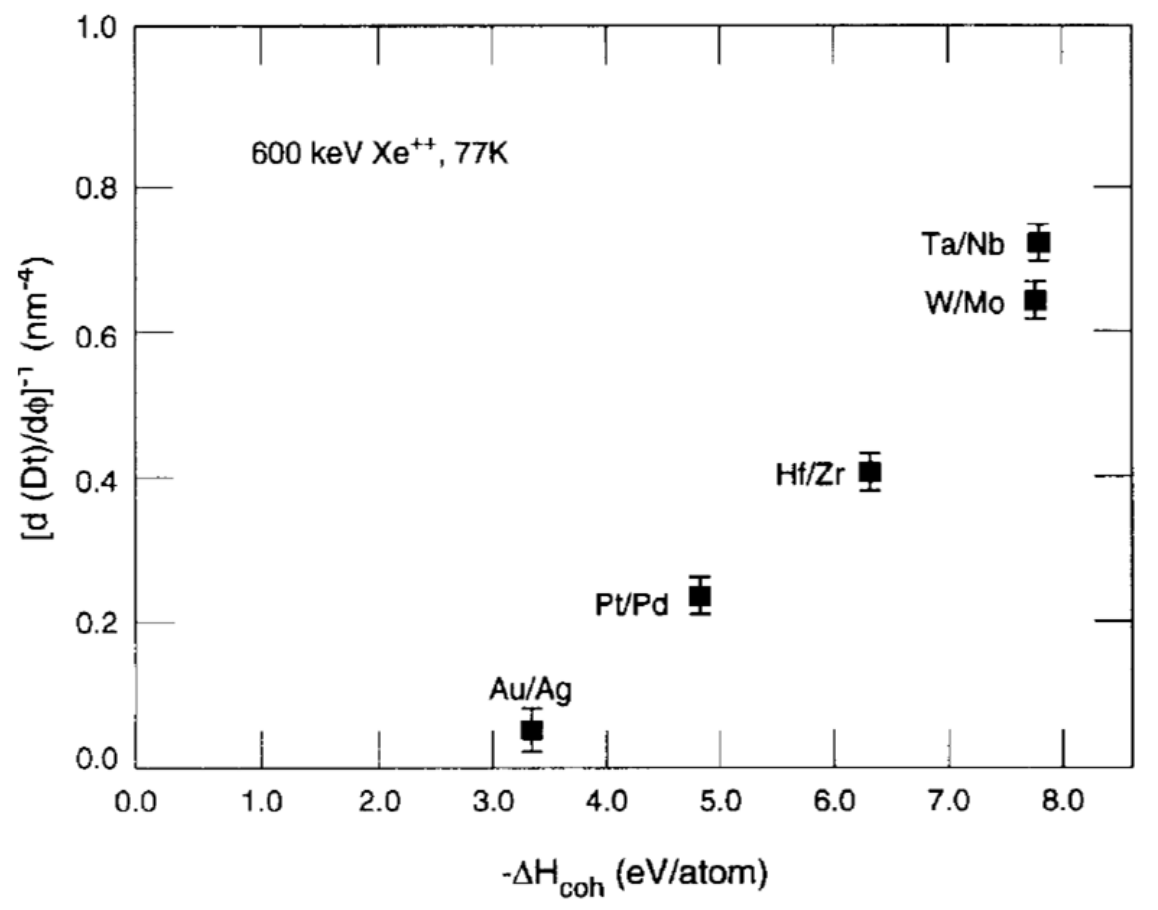

Figure 1-9: Relationship ion mixing and cohesive energy for different bilayer systems.

Finally, some models for mixing are reviewed. Most of the models presented in literature rely on the concept of thermal spike. A thermal spike is characterized by a small volume along the path of either the ion (global thermal-spike) or of one of the recoiled atoms (local thermalspike) where the majority of atoms are in motion and have reached approximately equipartition 
of kinetic energy. Within these volumes, high temperatures can be reached, and the material can experience transient diffusion processes which can happen in the liquid state. Contrasting this view the paper of Miotello and Kelly (36) opposes the idea that the chemically guided movement happens in the spikes, or between spikes if they are dense enough in the material to overlap. In their analysis, the mixing process ignores the ballistic motion due to inward directed recoils and the motion within a thermal spike. Mixing therefore can occur by three different mechanisms:

1. Random ballistic motion (much more important than the inward directed recoil motion)

2. Non guided defect motion

3. Chemically guided defect motion

Their analysis criticizes the thermal spike model for several reasons, among them the fact that chemically guided motion is observed even long (minutes) after the irradiation has ceased, while the thermal spikes only survive for short times ( 10-100ps). Chemically guided defect motion is implied even for system in which thermal spikes are probably not possible (36). Irradiations that lead to thermal spikes, in which the temperature is $\sim 10^{4} \mathrm{~K}$, should behave similarly when the temperature is changed (the relative difference would be very small on the cascade temperature).

\subsubsection{Temperature effects in ion beam mixing}

One of the factors that most influences ion beam mixing is temperature. To make clear the effect of temperature on the ion mixing, it is useful to refer to Figure 1-10.

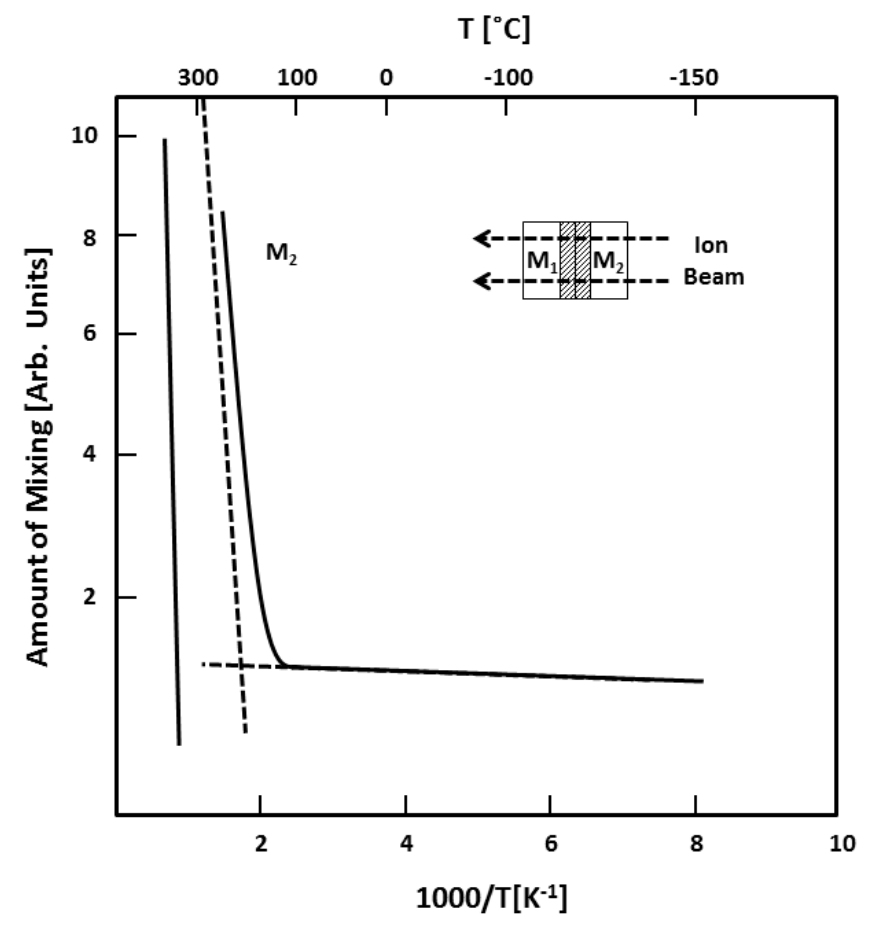

Figure 1-10: Influence of temperature on ion beam mixing. Increasing the temperature above a certain value causes the amount of mixing to change rapidly with changes in temperature (from Ref. (37)). 
On the left side of the graph, the mixing induced just by thermal effect is shown. Its behavior is described by an Arrhenius type curve, whose slope is determined by the activation energy of the process. The elbow-shaped curve represents ion mixing of a metallic bilayer in the presence of an irradiating species. Two different regimes can be identified: a temperature independent regime, at the low temperatures, in which the mixing of the system is not sensitive to a change in temperature, and a temperature dependent mixing regime, where temperature has a major effect on mixing. Again, the temperature dependent regime of the curve can be described with an Arrhenius type plot: therefore the difference between the two Arrhenius type plots is due to radiation enhanced diffusion. It must be noted that the low temperature mixing is due to ballistic mixing rather than a diffusion enhancement due to radiation (12). The critical temperature, $\mathrm{T}_{\mathrm{c}}$, sets the difference between the temperature dependent and the temperature independent mixing, and can be determined by the following approach (38). By defining an effective diffusion coefficient as:

$$
D=D_{\text {ballistic }}+D_{\text {rad }} e^{-Q / K T}
$$

Equation 1-19

where $D_{\text {ballistic }}$ is the temperature independent term, due to the ballistic mixing, and $D_{r a d} e^{-Q / K T}$ is the temperature dependent term, due to enhancement of diffusion by radiation. The $Q$ term is the activation energy. The temperature at which the two contributions are equal can be evaluated as:

$$
T_{c}=\frac{Q}{K\left[\ln \left(D_{\text {rad }} / D_{\text {ballistic }}\right)\right]}
$$

Equation 1-20

If the activation energy is assumed to be proportional to the cohesive energy through a constant C:

$$
T_{c}=\frac{C \Delta H_{\text {coh }}}{K\left[\ln \left(D_{\text {rad }} / D_{\text {ballistic }}\right)\right]}
$$

A linear relationship is indeed found between the critical temperature and the cohesive energy, as shown in Figure 1-11. The data are taken from different sources in which different ion species, energies, doses, and dose rates are used. Given the linearity of the results with respect to cohesive energy, these parameters can be safely assumed to be less important than the cohesive energy on the value of the critical temperature of the system irradiated. 


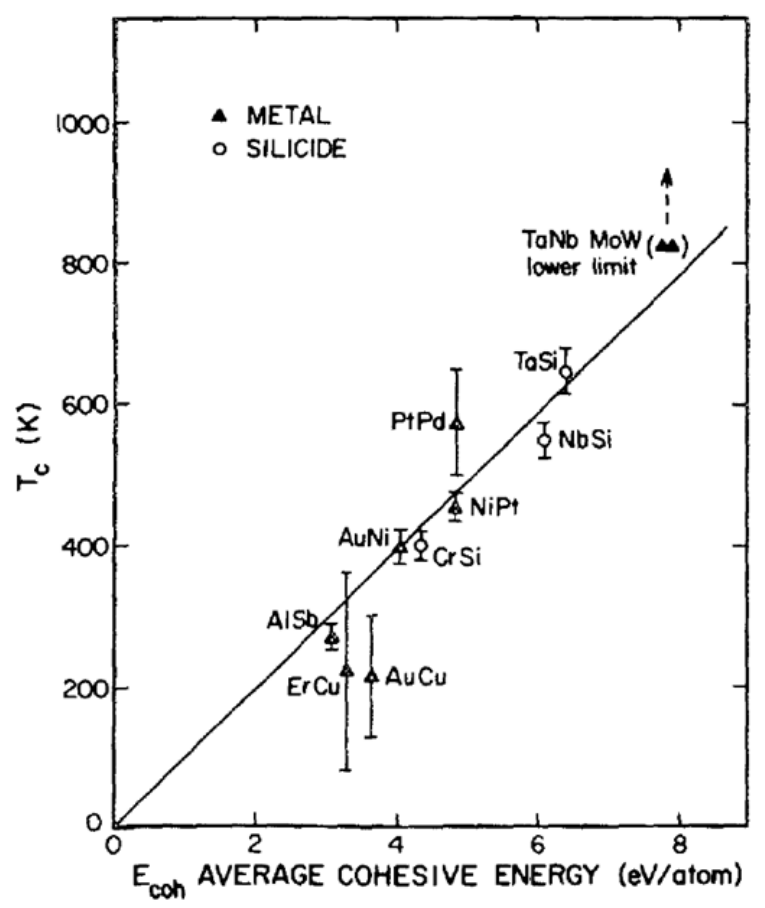

Figure 1-11: Correlation between the average cohesive energy and the critical temperature (from Ref (38)).

\subsubsection{ODS steels and interfaces}

Ferritic/martensitic (F/M) steels provide better void swelling resistance under irradiation than austenitic steels, but have poor creep strength at high temperature (over $600^{\circ} \mathrm{C}$ (39)). To overcome this limitation, oxide dispersion strengthened F/M steels have been developed. Nanofeatured, oxide-dispersion-strengthened (NF-ODS) steels are complex alloy systems which are typically fully ferritic or martensitic with a dispersion of nano-sized complex oxides within the matrix. These complex oxides are typically referred to as nanoclusters. The fine dispersion of nanoclusters within the ferritic-matrix serve as point defect annihilation sites and pinning sites for dislocation motion and serve to improve the high temperature tensile and thermal creep properties over more traditional F/M steels (40; 41; 42; 43). Additionally, the nanoclusters are expected to promote recombination of irradiation-produced point defects and to trap transmutation-produced He in small, high-pressure bubbles (44). Figure 1-12 shows electron micrograph series, in which shows the small nanoclusters are visible.

Stability of compositionally different ODS steels have been studied after irradiation with different particles (heavy ions (6) (45), neutron (46), electrons (47), dual beams (48)). It was observed that the oxide particles are stable at high temperature irradiation $\left(600^{\circ} \mathrm{C}\right)$ and their dissolution is observed only at very low temperatures. In fact, when a $14 \mathrm{YWT}$ (Fe-14 wt\%Cr-3 wt\%W-0.4 wt\%Ti-0.25 wt\% $\mathrm{Y}_{2} \mathrm{O}_{3}$ ) ODS steel was irradiated with $5 \mathrm{MeV} \mathrm{Ni}^{++}$ions to $100 \mathrm{dpa}$ at low temperature $\left(-75{ }^{\circ} \mathrm{C}\right)$ the clusters were completely dissolved into the matrix. However, after annealing under vacuum at $850^{\circ} \mathrm{C}$ for 5 hours, the nanoclusters reassembled with a size distribution similar to the unirradiated specimen. Therefore the stability at high temperature is 
deemed to be caused by a mechanism in which the solute atoms are likely to diffuse and recombine (49).
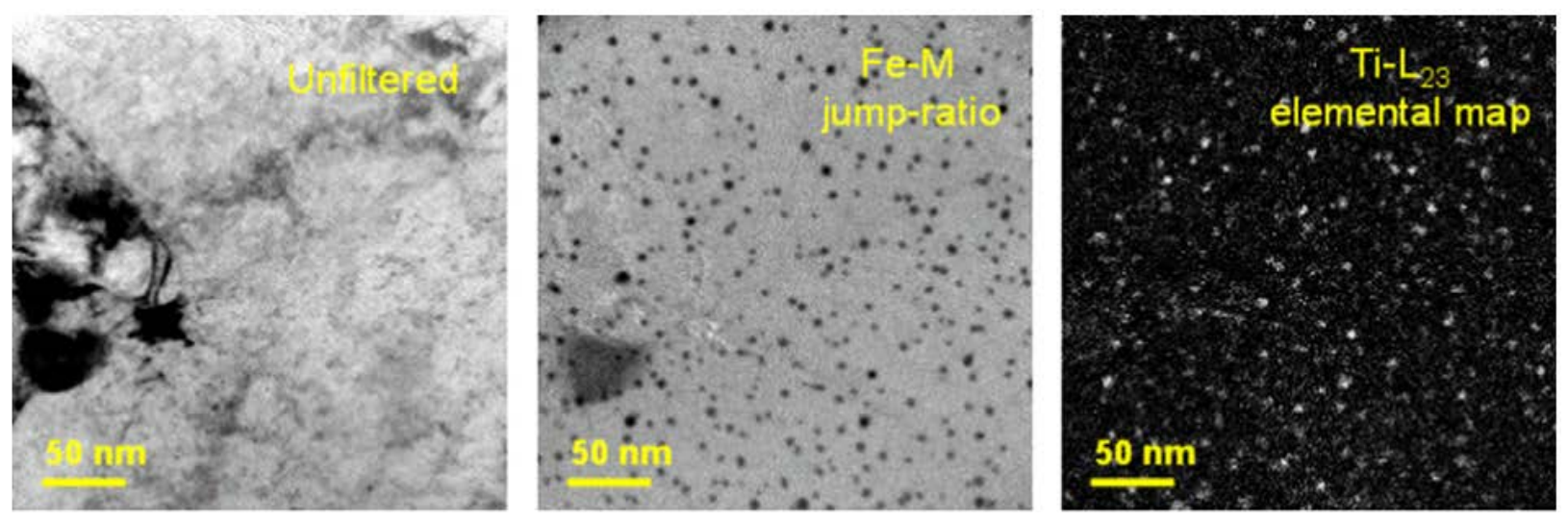

Figure 1-12: Energy Filtered TEM images of a typical ODS steel. The black dots in the jump-ratio indicate regions depleted in iron, while the white spots in the Ti-L23 maps indicate regions enriched in titanium, i.e. the nanoclusters (from Ref. (49))

Since the nanoclusters play a fundamental role in the behavior of these steels at high temperature and under radiation, their own stability, which rely on the stability of the particlematrix interface is critically important and must be understood. The small size of the nanoclusters makes it difficult to experimentally study the stability of the interface but has been done in a few studies (8). By depositing thin films of yttrium, titanium, yttrium oxide and titanium oxide an ideal system, which has an artificially enlarged interface between the nanoclusters and the matrix, can be created as shown in Figure 1-13. Once the system is formed, it can be irradiated with heavy ions and the stability of the interface against radiation can be evaluated.
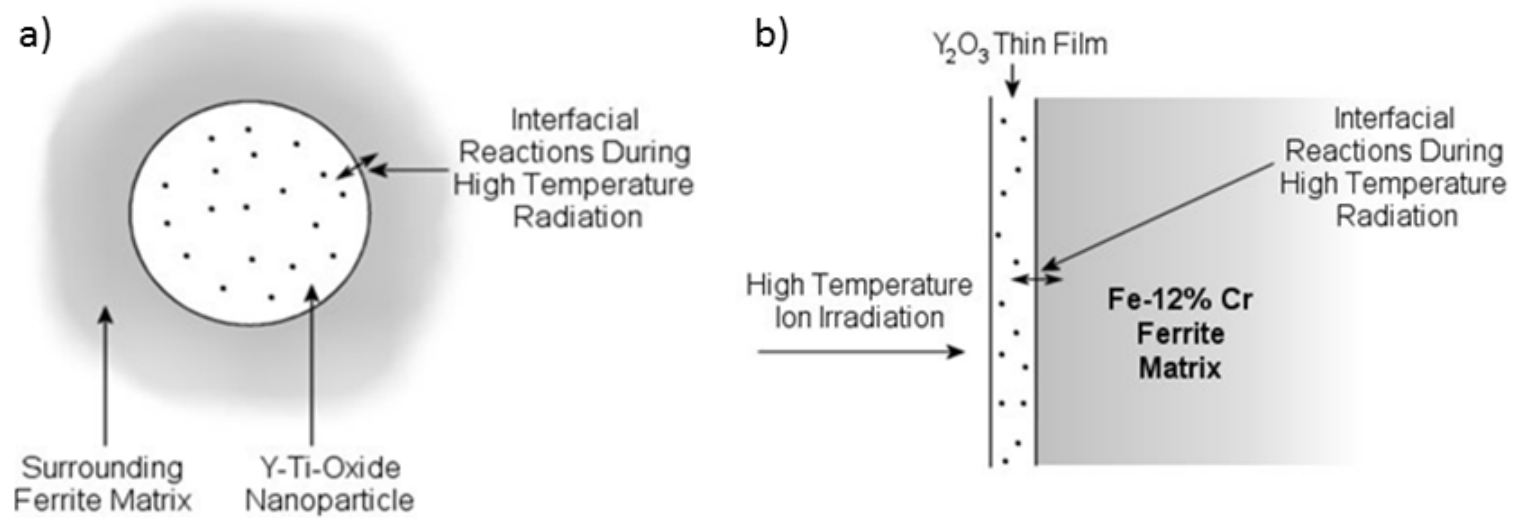

Figure 1-13: Schematic illustration of a) nanometer sized $Y$-Ti-O nanocluster embedded in a ferritic matrix and b) experimental analog involving high temperature ions irradiation of a $\mathrm{Y}_{2} \mathrm{O}_{3}$ thin film. 


\section{CHAPTER 2 \\ Microstructural Evolution in Multilayered Systems}

\subsection{Multilayered systems}

\subsubsection{Radiation damage effects in multilayered systems}

Irradiation can alter the stability of phases and the irradiation altered phase stability is considered as "any microstructural change that would not have occurred without an irradiation field, all other conditions being the same” (50). Following the classification of Hudson (51) the different irradiation enhanced processes are, diffusion, dissolution of precipitates, disordering reordering, nucleation, and formation of new or non-stable phases, segregation and morphological changes. The amount of energy introduced during irradiation is so large and that despite some dissipation during the recombination of interstitials and vacancies, there is still enough to produce non-equilibrium phases (50).

In the last decades, several studies have shown that an effective way of improving the radiation resistance of a material to radiation can be achieved by increasing the fraction of interfaces. Experimental results have shown that nanocrystalline materials containing large fraction of GBs (nickel and copper (52), gold (53), ZrO2 (54), nanograined steels (55)) exhibit an improved resistance to radiation as compared to their crystalline counterparts. This is corroborated by simulations performed on copper (56). Moreover, some systems made up of layered metallic compounds show an increased radiation tolerance (57) (58). Finally, oxide dispersion strengthened (ODS) steel exhibit higher tolerance to radiation damage than conventional steel, an effect attributed to the interface between the nanoparticles and the matrix. Therefore is extremely important to gain a better understanding of the behavior of interfaces, especially in the case of those systems exposed to high radiation field, typical of the environment of a nuclear reactor, in order to be able to develop a new class of radiation resistant materials.

Artificial multilayers are metastable due to the high content of interfaces and the specific methods of deposition. Therefore, the stability of multilayers under thermal and ion irradiation treatments has important consequences for their applications (59). Several studies have been published on metallic layered systems subjected to high temperature and irradiations. Although the systems studied in literature are composed of alternating thin layers of two different materials and, therefore, differ from the system under investigation in this work (Fe-12\%Cr alloy coated with thin films), some of the ideas and the results found in these papers are useful to interpret the results that will be presented in the later chapters.

\subsubsection{Radiation resistant multilayered systems}

Different aspects must be taken into consideration to evaluate the response of multilayered systems to radiation and, therefore, different systems irradiated under the same conditions can show completely different behaviors. One of the parameters which determine the stability of the multilayers to radiation is the individual layer thickness. For example, when 
sputter-deposited $\mathrm{Cu} / \mathrm{V}$ nanolayered films with different individual layer thickness, $\mathrm{h}$, were subjected to $50 \mathrm{KeV}$ helium ion irradiation at room temperature to a dose peak of 6 dpa, it was observed that decreasing the individual layer thickness, the average helium bubble density, the lattice expansion, and the magnitude of radiation hardening were all found to decrease (60);. In particular, the radiation hardening becomes negligible for $\mathrm{h}$ equal to or less than $2.5 \mathrm{~nm}$. Moreover, the morphology of the thin layers does not seem to be affected by the radiation, as can be seen from Figure 2-1.
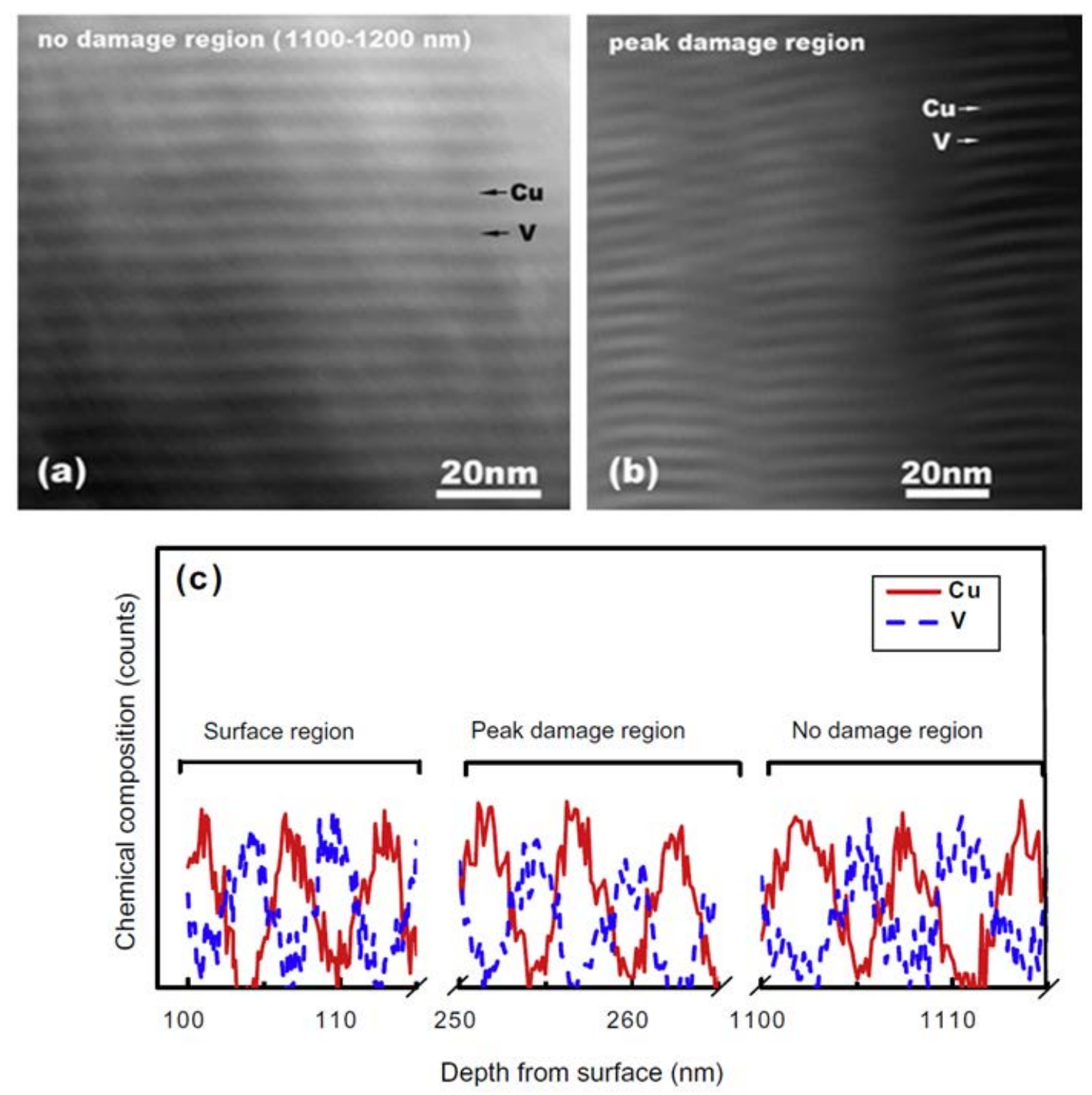

Figure 2-1: STEM images of the helium implanted $2.5 \mathrm{~nm}$ thickness $C \mathrm{Cu} / \mathrm{V}$ multilayers. a) Surface region (no damage), b) At the damage peak region (200-325 nm) c) EDS chemical analysis. The individual layer morphology is maintained after helium implantation (from Ref. (60)).

For average helium concentrations less than 0.28 at. \%, helium bubbles were not detectable in $\mathrm{Cu} / \mathrm{V} 50 \mathrm{~nm}$ multilayer. This is several orders of magnitude greater than the helium solubility in 
lattices of metals, typically at ppm level. An even greater threshold of 1 at. \% of helium was identified in $\mathrm{Cu} / \mathrm{V} 2.5 \mathrm{~nm}$ multilayers. Consequently, $\mathrm{Cu} / \mathrm{V}$ nanolayers possess enhanced radiation tolerance, i.e., reduction of swelling and suppression of radiation hardening, compared to bulk $\mathrm{Cu}$ or $\mathrm{V}$. Their radiation resistance is deemed to be determined by their positive heat of mixing ( $\sim 5 \mathrm{~kJ} / \mathrm{mol})$, which, as discussed in 1.3.1.3 impede/counterbalance intermixing effects between the different layers during radiation.

A similar behavior was also observed in $5 \mathrm{~nm}$ immiscible $\mathrm{Cu} / \mathrm{Mo}$ multilayer systems irradiated at room temperature with $100 \mathrm{KeV}$ helium ions (61) and also in $\mathrm{Cu} / \mathrm{Nb}$ multilayers. The $\mathrm{Cu} / \mathrm{Mo}$ interfaces act as effective sink the point defects generated during radiation and helium atoms, which have high solubility in this system. The $\mathrm{Cu} / \mathrm{Nb}$ system is characterized by a positive enthalpy of mixing and has a high lattice misfit between the two constituent of the sample. Here there is a great ability to reduce the point defect concentration (58). Atomistic modeling shows that at these interfaces pairs of extended jogs formed by misfit dislocation along the interfaces become sinks for point defect formation (62) as shown in Figure 2-2.

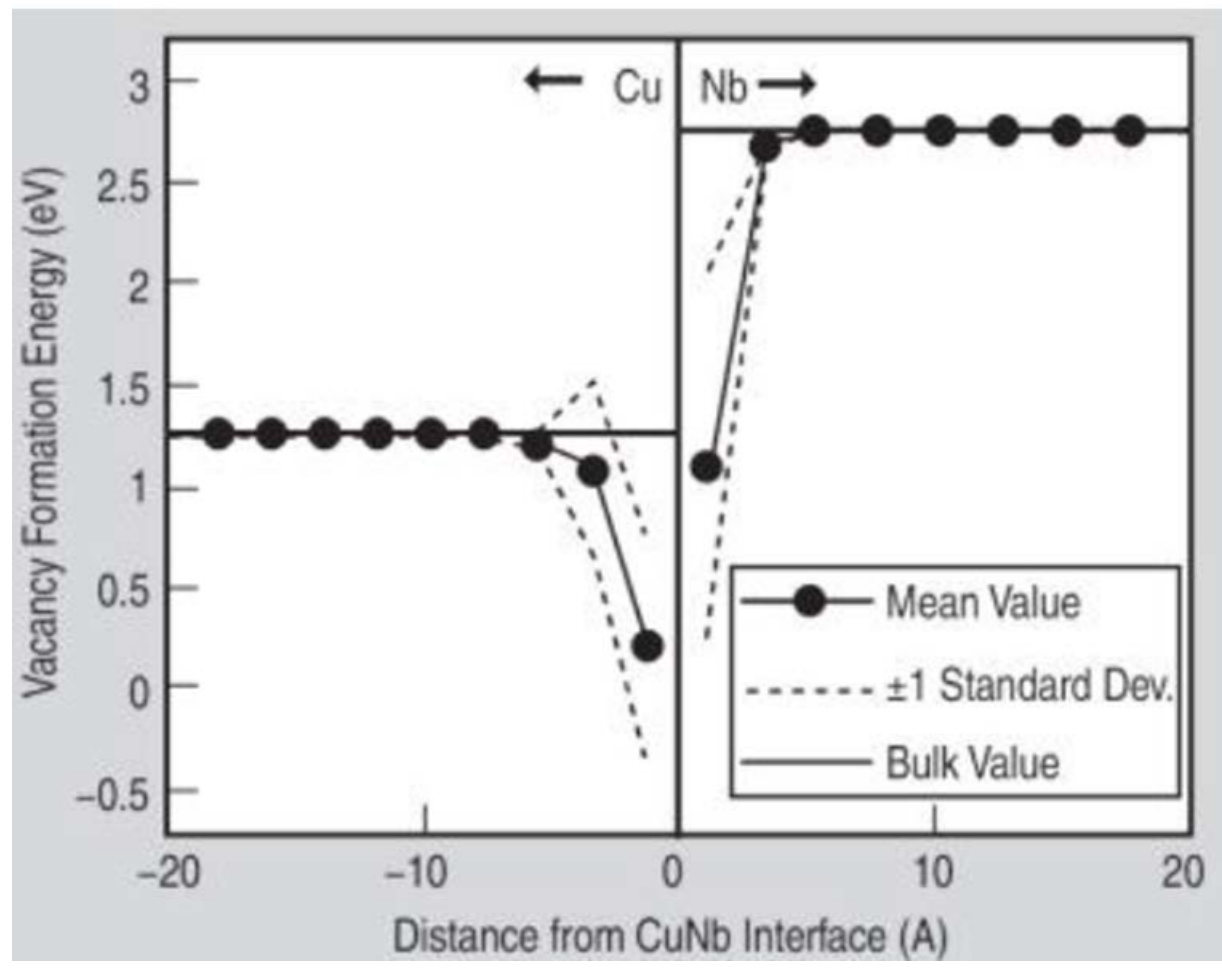

Figure 2-2: Formation energies of point defects at the $\mathrm{Cu} / \mathrm{Nb}$ interface, compared to bulk $\mathrm{Cu}$ and $\mathrm{Nb}$. At the interface the formation energy is sensibly lower (from Ref (58)).

On the other hand, when Fe and W are sputter deposited to form a multilayer composite and irradiated with $100 \mathrm{KeV} \mathrm{He}$ ions to a fluence of $6 \times 10^{16} / \mathrm{cm}^{2}$, at the peak damage region, a large number of helium bubbles were observed without any clear resolution of the layer structures. The average helium bubble size was $\sim 1-2 \mathrm{~nm}$ in diameter. The removal of layer interface is due to the fact that $\mathrm{Fe}$ and $\mathrm{W}$ are chemically miscible and can form several intermetallic phases according to their phase diagram. Therefore the enthalpy of mixing plays an important role in the evolution of layered systems after radiation. However, even though the 
system is chemically disrupted after irradiation, the magnitude of hardness variation depends on the individual layer thickness, and hardening is observed just in specimens with individual layer thickness of $\geq 5 \mathrm{~nm}$ (63).

However, the miscibility of the system is not the only parameter which controls the stability of the multilayer after irradiation, even though usually the ability to retain the layered morphology in immiscible systems is explained based on the enthalpy of mixing which favors de-mixing. For example, $\mathrm{Al}$ and $\mathrm{Nb}$ have a negative heat of mixing and when they have been irradiated $100 \mathrm{KeV}$ helium ions to a dose of $6 \times 10^{16}$ ions $/ \mathrm{cm}^{2}$, XTEM and STEM studies showed that nanolayers structures are retained in all films even in the peak radiation damage region (Figure 2-3).
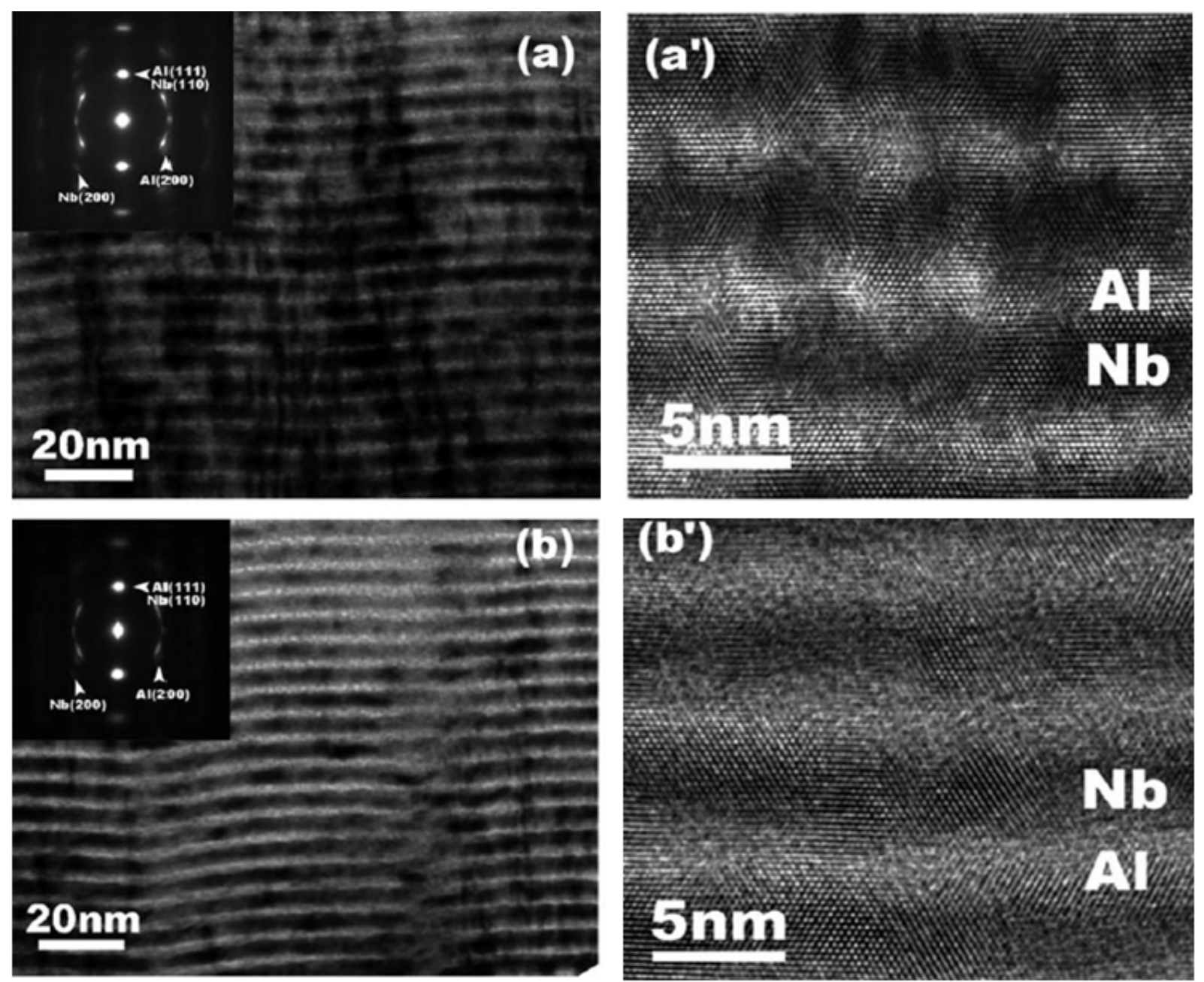

Figure 2-3: XTEM micrographs of AI/Nb 2.5 multilayers after $100 \mathrm{KeV}$ helium implantation to a dose of $6 \times 10^{16}$ ions $/ \mathrm{cm}^{2}$ in the a) the surface region and b) the peak damage region: the individual layer structure is retained after irradiation (from Ref

(64)).

Examination of the $\mathrm{Al} / \mathrm{Nb}$ phase diagram indicates three different intermetallic compounds (65). Thermal stability studies of this system have revealed that different phases are 
formed in a sequential manner, depending by the annealing time, and the annealing temperature (66; 67; 68). The phase that forms first during irradiation is $\mathrm{Nb}_{3} \mathrm{Al}$. This observation differs from the observations in the thermal annealing studies of $\mathrm{Al} / \mathrm{Nb}$ multilayers, where the first phase to form is $\mathrm{NbAl}_{3}$. This shows that phase transformation induced ion mixing can differ from equilibrium thermal annealing processes. STEM studies suggest that the $\mathrm{Nb}_{3} \mathrm{Al}$ phase forms along layer interface due to radiation induced ballistic mixing. The phase formed under irradiation is characterized by more densely packed crystallographic structure as compared to $\mathrm{Al}$, with much stronger interatomic bonds and therefore is suggested that this phase layer prevents further intermixing of $\mathrm{Nb}$ and $\mathrm{Al}$ during helium implantation. $\mathrm{Nb}_{3} \mathrm{Al}$ may then act as a barrier to the interdiffusion, facilitating the retention of layered structure.

\subsubsection{Fe/Ti system}

There is literature about the thermal stability of layered metallic system, however little is still known about the interdiffusion mechanism and the stability of the interface in these systems (69). Also the microstructure of the intermixed layer has still to be investigated into detail. Multilayered crystalline $\mathrm{Fe} / \mathrm{Ti}$ systems prepared by sputter deposition, with individual layer thicknesses ranging from 5 to $40 \mathrm{~nm}$ were studied in terms of thermal $(70 ; 71 ; 69)$ and irradiation stability (72; 73; 74). In terms of their thermal stability, although not all the studies found in literature are in agreement, a general trend can be outlined. Annealing in vacuum between 523 and $823 \mathrm{~K}$, causes the dissolution of titanium into the $\alpha$-Fe sublayer to form a Fe-Ti bcc solid solution. Increasing the time of exposure, or the temperature, causes the formation of the intermetallic FeTi compound in the Fe-Ti solid solution (69; 71; 70), as seen in Figure 2-4.

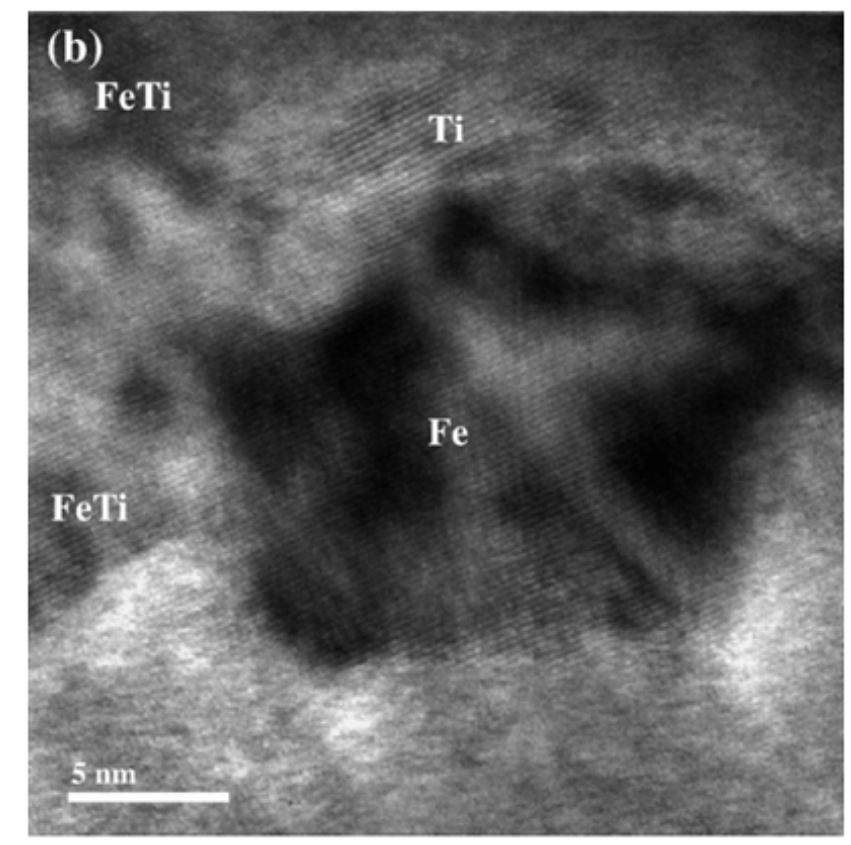

Figure 2-4: Cross sectional high resolution TEM images of the Fe/Ti multilayers $19 \mathrm{~nm}$ thick submitted to thermal annealing at $450^{\circ} \mathrm{C}$ for $1 \mathrm{~h}$ (from Ref. (70)). 
The parameters which govern the response to radiation are the thickness of the individual layers and the dose (72), the temperature and the presence of contamination ( $\mathrm{C}$ and $\mathrm{O})$ in the asdeposited layers (73). Irradiation performed with low energy $\mathrm{Ar}^{2+}(200 \mathrm{KeV})$ or $\mathrm{Kr}^{2+}(350 \mathrm{KeV})$ below $50^{\circ} \mathrm{C}$ (72) result in an increase of the relative fraction of the bcc-FeTi phase in the lower dose regime with a linear to square root dependence on dose. This is due to the mass transport during mixing, which is probably occurring due to a random walk mechanism, with chemical forces playing just a minor role. A further increase of the dose causes first, the formation of an amorphous phase and later its decrease with a partial restoration of the bcc-FeTi fraction. The presence of carbon greatly reduces the mixing of the layers and reduces the ability to form an amorphous phase (73).

\subsubsection{Pt(Fe)/C(Fe)}

Studies involving the irradiation stability of Pt/C multilayers, in which Fe ( $15 \%$ at.) is introduced, have been studied in literature (59; 75; 76). In these studies, alternating layers of Pt and $C$ with individual thickness of $1.6 \mathrm{~nm}$ and $2.6 \mathrm{~nm}$ were deposited usually on float glass substrates and irradiated with $2 \mathrm{MeV} \mathrm{Au}{ }^{+}$ions at different fluences $\left(10^{14}\right.$ to $\left.10^{15} \mathrm{ions} / \mathrm{cm}^{2}\right)$. In (75), in the as-deposited condition, the diffraction patterns and the HRTEM images show that the Pt layers are polycrystalline, while the $C$ is amorphous (59). For low fluence exposures (up to $3 \times 10^{14}$ ions $/ \mathrm{cm}^{2}$ ) ballistic mixing across the Pt/C interfaces increases interface roughness; Pt atoms are pushed into $C$ layers and vice versa and an expansion of the multilayer period is also observed. For higher fluences, surface and interface roughness decrease due to de-mixing across the $\mathrm{Pt} / \mathrm{C}$ interfaces. As expected, this is explained in terms of ballistic and chemically guided atomic movements. Ballistic effects, which are always present, act as a mixing mechanism during the initial stages of the process, however, as the irradiation dose is increased, this process is counterbalanced by a chemically driven process, which results in de-mixing of the two materials.

When the collision cascades spatially overlap, the atomic relocations can take place over extended regions and the role of chemical driving force is enhanced. This favors atomic migration over extended distances and can eventually cause phase segregation in the case in which the components are immiscible. The heat of mixing between $\mathrm{Pt}$ and $\mathrm{C}$ is strongly positive $\left(\Delta \mathrm{H}_{\mathrm{m}}^{1: 1}=52.7 \mathrm{Kj} / \mathrm{mol}\right)$ so there is tendency for de-mixing, because the atomic relocations are not anymore completely random, but they are indeed chemically biased. The thermally activated and the diffusional processes, enhanced by irradiation, tend to restore the system to its equilibrium state.

Migration of iron atoms upon irradiation of the system is preferentially directed outward and from $\mathrm{C}$ to Pt layers. It possible to rationalize this migration on the basis of the heat of mixing being immiscible for Fe-C, yet miscible for Fe-Pt (59). 

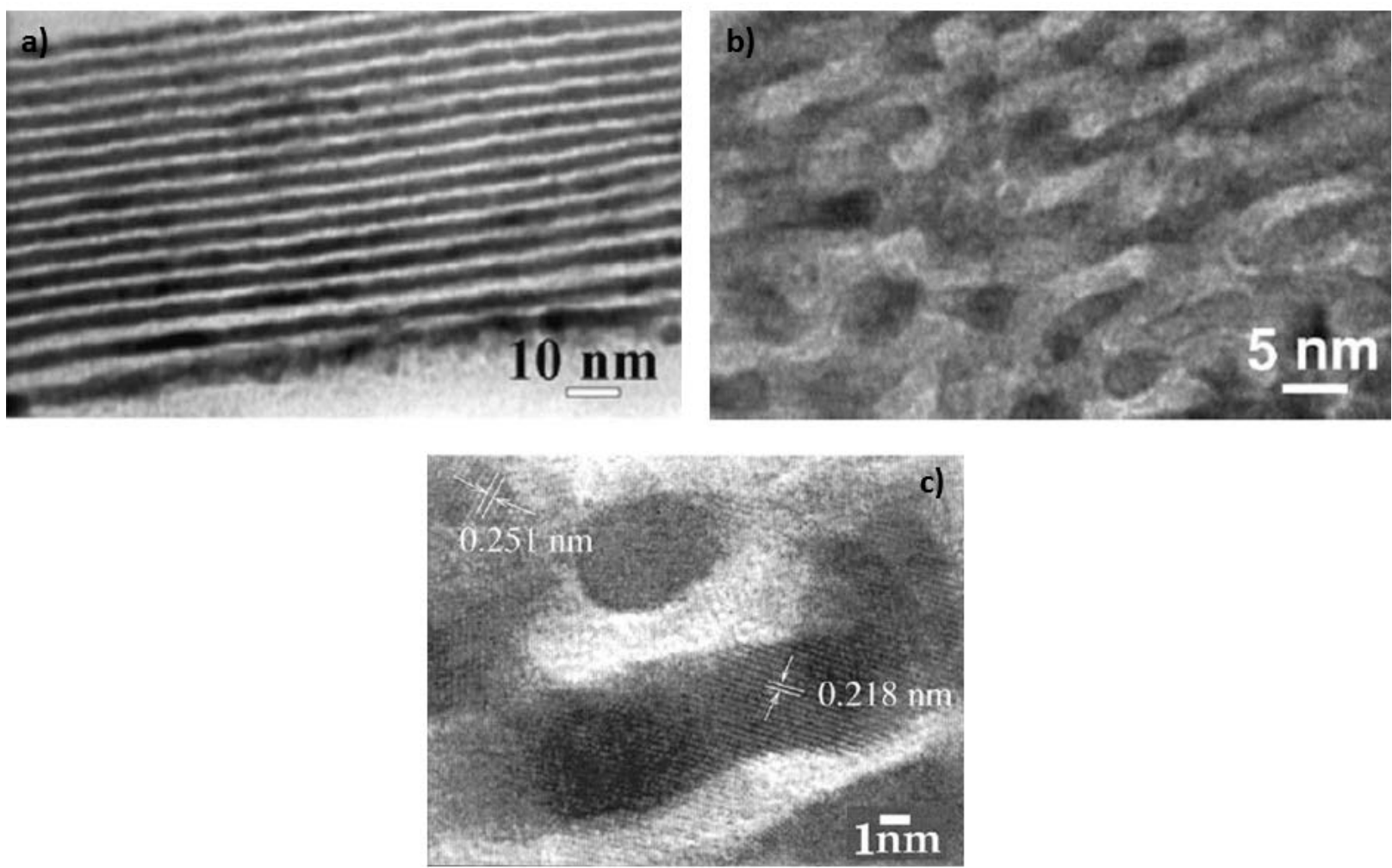

\section{Figure 2-5: Cross sectional TEM images of the irradiated Pt(Fe)/C(Fe) samples a) $5 \times 10^{14}$ ions/cm b) $1 \times 10^{15}$ ions/cm c) HRTEM of a nanoparticle in b) (from Ref. (59; 75)).}

It is also possible to predict the directionality of the migration of iron atoms which was found to be directed outwards. For binary alloys, surface segregation can be predicted based on the value of the heat of segregation. For large heat of segregation values, segregation is predicted to occur. But the heat of segregation is not the only value that must be taken into account and oxygen in the irradiation chamber can play a decisive role. By comparing the segregation of iron in the Pt-Fe solvent-solute system after annealing at $1200^{\circ} \mathrm{C}$ in ultra-high vacuum conditions and after annealing at $700^{\circ} \mathrm{C}$ in $10^{-7}$ Torr of $\mathrm{O}_{2}$ (77), no segregation of iron was observed in the ultrahigh vacuum experiment, while iron was observed to segregate to the Pt surface in the poorer vacuum experiment. It is therefore speculated that oxygen is deemed to responsible of the outward segregation in the system.

\subsubsection{Fe-Al thin films}

Fe-Al thin films were studied after being subjected to annealing, ion beam mixing (78). In the work of Vasconcellos et al. (78) a thin film of iron (100nm), followed by a thin film of aluminum $(500 \mathrm{~nm})$ were deposited on a silicon substrate. Thermal annealing was performed in a high vacuum furnace between 290 and $870 \mathrm{~K}$, while $\mathrm{Ar}^{+}$ions of $240 \mathrm{KeV}, 480 \mathrm{KeV}$ and $900 \mathrm{KeV}$ were used for irradiations performed at 77, 300 and 500K. Even though the mechanisms responsible for atomic movement in the two processes are different, striking similarities were observed. The behavior of interdiffusion and the phases identified were also similar (Figure 
2-6). The general observation is that irradiation has the effect of lowering the temperature necessary to reach certain interdiffusion or reaction.

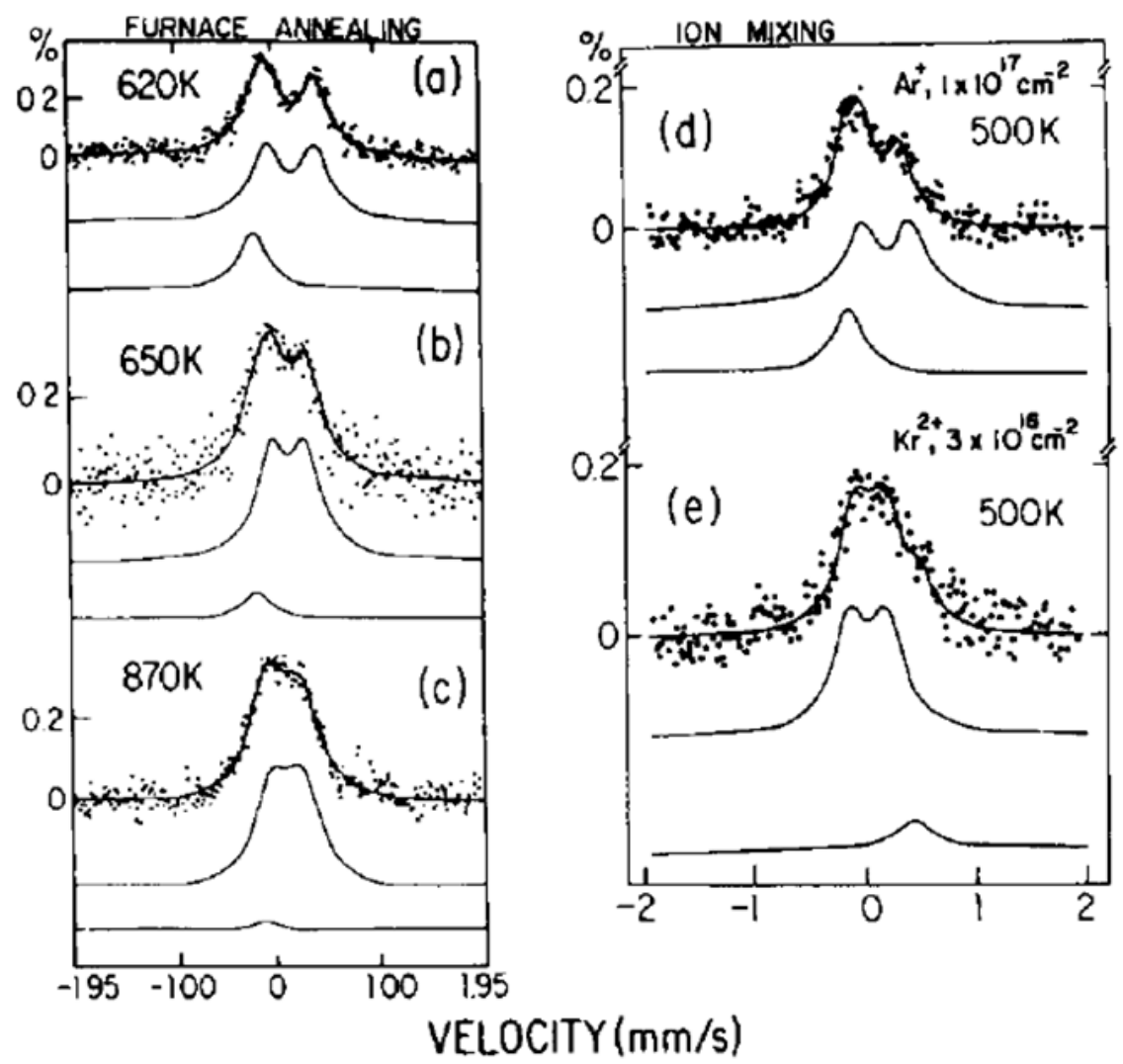

Figure 2-6: CEMS analysis of the Fe-Al thin film bilayer furnace annealed in high vacuum (a and $b$ ), and $\mathrm{Ar}^{+}$and $\mathrm{Kr}^{+}$ion beam mixed ( $c$ and $d$ ).

The Continuous Emissions Monitoring Systems (CEMS) data show that annealing at $600 \mathrm{~K}$ to $620 \mathrm{~K}$ causes the formation of the crystalline intermetallic phase $\mathrm{Fe}_{2} \mathrm{Al}_{5}$, the same one that is observed after ion beam mixing at $500 \mathrm{~K}$. An annealing temperature of $650 \mathrm{~K}$ leads to the formation of $\mathrm{FeAl}_{6}$, the same as observed after irradiation with $\mathrm{Kr}^{+}$at $500 \mathrm{~K}$ (which has $\mathrm{F}_{\mathrm{d}}$ greater ta $\mathrm{Ar}^{+}$). Also the diffusion profiles as revealed by RBS show similarities.

\subsection{Formation of nanoclusters}

In recent years, nanoclusters have attracted much interest due to their unique properties, which differ from the bulk materials. Nanoclusters can be unique in term of their structural, optical, electronic, thermal, mechanical and magnetic structure (79). Nanoclusters have been produced by accelerated ion beams (80) and can be formed with a beam of ions by direct implantation of impurity atoms into the system (81) (82) or by irradiation of a multilayered 
system (75). Synthesis of nanostructures and their modifications using ion beams has been investigated by Heinig et al. (80), where a scheme was developed for ion-beam synthesis of nanostructures.

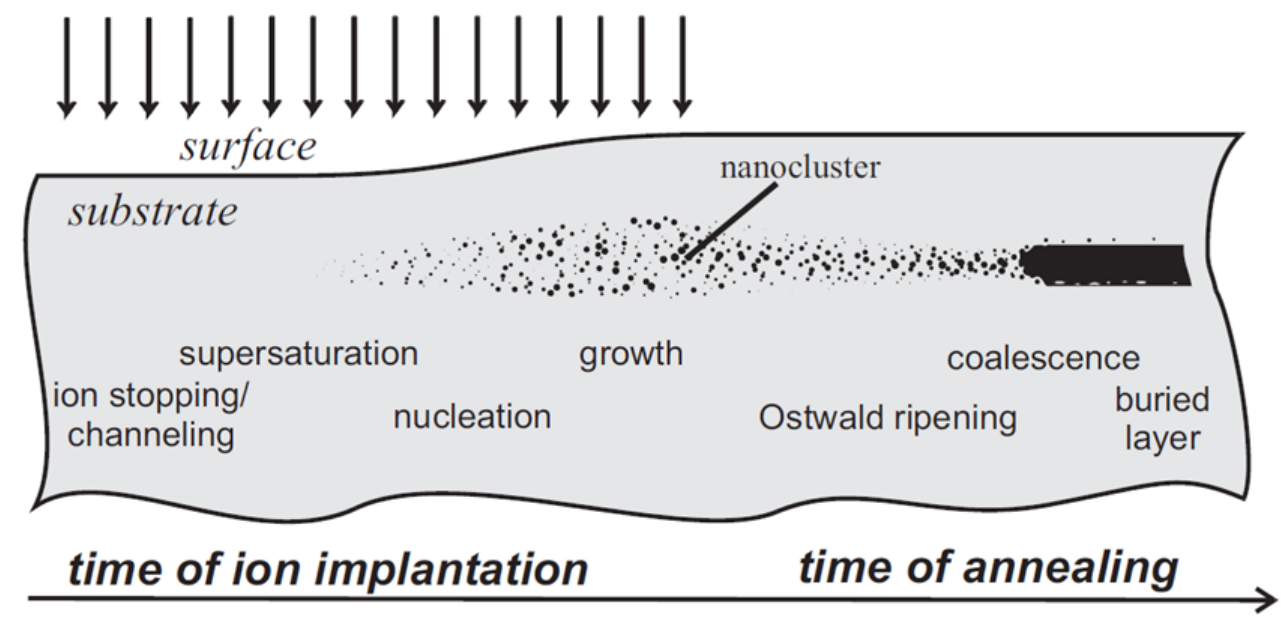

\begin{abstract}
Figure 2-7: Scheme for ion beam synthesis of nanostructures. When a material is implanted with high dose is supersaturated with impurity atoms is observed. The nanoclusters nucleate and grow during ion implantation if they have enough mobility, otherwise during subsequent annealing (from Ref (83)).
\end{abstract}

On the other hand, ion irradiation can also affect embedded nanoclusters. Recoils produced during the collision cascade can knock out atoms from the nanoclusters and deposit them in the matrix. Depending on their mobility in the environment, their concentration under steady state conditions is determined in by the balance of detachment and attachment processes at the interface. Thermally activated diffusion and thermally activated jumps over a reaction barrier are necessary for the attachment, which, therefore, is a thermally activated process if one neglects ion-irradiation-enhanced diffusion. Relocation of atoms from the nanoclusters to the surrounding matrix can be either a thermally activated process or can be caused by collision with the recoiling atoms producing by the cascade. While at high temperature the thermally activated detachments will dominate, at low temperature they will become temperature-independent, i.e. detachments will be dominated by ion beam mixing (80).

\title{
2.3 Thin film depositions
}

Thin films are deposited onto bulk materials to obtain properties that are unattainable or not easily attainable in the substrate alone (84). A major classification discriminates between thin film deposition, in which individual molecules are deposited on the substrate, and thick film deposition, which involves the deposition of particles. Examples of thick film deposition are painting, spin on glass coating, plasma spraying and silk screening. Those techniques are important and relatively inexpensive, but they do not allow the control of the material quality of the thin film techniques (84). In the following section, only the process involving thin film deposition from vapor phase (the one in which molecules from the target are vaporized) will be described. These techniques are particularly important to present research.

All thin film deposition is made up of different steps: 
- A source of the material to be deposited is provided

- The material has to be transported onto the substrate

- The deposition takes place

The source can be a solid, liquid or a gas. In the case of the solid, the particles of the source must be brought to vapor phase (e.g. physical vapor deposition, PVD), and this can be done either by heat, bombardment with electrons, photons (ablation) or positive ions (sputtering). (84). Once the particles are evaporated the second step of the process, the transport, takes place. The major issue during this step is the uniformity of arrival rate on the substrate which in turn depends on the medium used for the transport process which can be vacuum, fluid or plasma. Many thin film processes operate in plasma, which contain a great deal of energy and can be set at different pressures, so it can behave either as fluid or high vacuum medium. The $3^{\text {rd }}$ step is the actual deposition on the substrate. This step is influenced by the previous step factors (source and transport) and the condition of the surface coated. The most important factors related to the surface to be coated are: the roughness, the level of contamination and the degree of the chemical bonding with the arriving material and the reactivity, which refers to the probability that the arriving species will interact with the system and will be incorporated into the film (sticking coefficient). The last factor is the energy of the arriving particles, which has profound effects in the reactivity of the arriving material and on the composition and structure of the film. The basic source of energy is the substrate temperature, but other particles can be used to increase the energy of the incoming particles (photons for photo assisted deposition or positive ions, which carries a large amount of energy and which is present in most plasma processes). The three deposition factors described above are of importance with the arriving flux to determine the structure and the composition of the thin film.

\subsubsection{Sputtering}

Sputtering is the erosion of a material by the interaction with energetic positive ions. Usually, sputter deposition devices consist of a deposition chamber, a supply gas, pumps, a DC/RF voltage power supply, a quartz crystal thickness film, a cathode and a deposition rate controller. Deposition is performed at pressures typically lower than $2 \times 10^{-6}$ Torr. A very common sputtering gas is argon, but sometimes a combination of gases may be used for reactive sputtering. The process of sputtering happens when the cathode is powered with a negative DC power supply. By the application of the DC voltage, the electrons in the system are accelerated away from the target and collide with the molecules of the gas in the chamber, initiating and sustaining the plasma. The now positively charged particles are attracted toward the cathode. From the collisions of the energetic positive charges of the plasma with the target, neutral species (atoms, clusters or molecules) are emitted from the surface and deposit on the surface of the substrate. A schematic of the process is shown in Figure 2-8. 


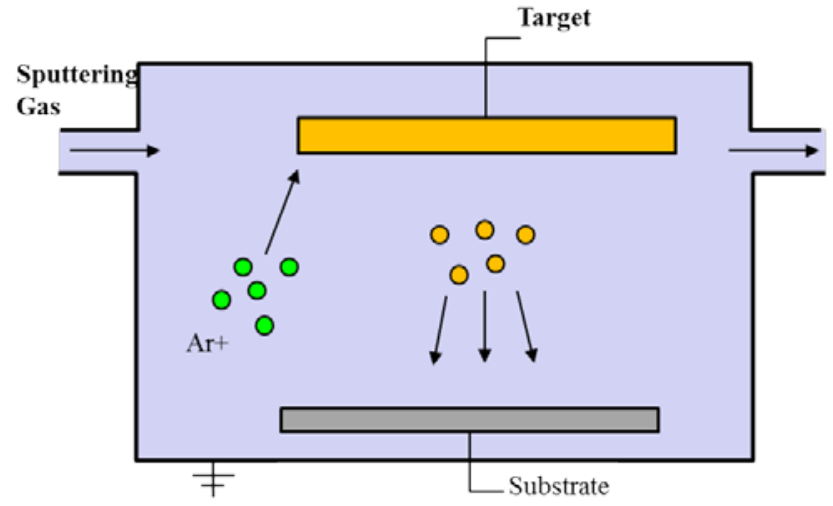

Figure 2-8: DC sputtering process: the target is negatively polarized with a DC voltage. The electrons in the system will be accelerated away from the target and will collide with the molecules of the gas filling the chamber. This interaction will ionize the gas molecules and the now positively charged particles will attract toward the cathode and the sputtering process will proceed.

To determine the thickness of the deposited film a quartz crystal microbalance, or QCM is used with a film thickness monitor which works on the following principles (85) (86). When a RF voltage is applied to a quartz crystal, this will oscillate at its natural frequency. At a given temperature, this frequency will be function of the mass. While the film thickness builds up, the oscillating frequency will change (increasing the mass it will lower) and this signal can be converted by the system to a thickness measurement.

The film thickness monitor works on the underlying assumption that any change in the resonance frequency is solely a result of film build-up, but unfortunately, this may not be true and thermal gradients or mechanical stresses can change the vibrating frequency. The parameters that need to be known for accurate thickness measurement are the density of the cathode and the Z-ratio. For some compounds the value of the Z-ratio is not known and, moreover different deposition condition can lead to different values. Therefore, before starting the deposition, a calibration of the system needs to be performed. 


\section{CHAPTER 3 \\ Experimental Design and Sample Preparation}

\subsection{Samples}

\subsubsection{Model Fe-12\%Cr Steel}

A 12 wt. \% Cr model alloy, with a nominal representative of a class of widely used ferritic steels, was purchased from the Sophisticated Alloys, Inc (Butler, PA) and used as a substrate for the thin film deposition. This model alloy was used due to the simplified chemistry and negligible second phases such as carbides. This facilitated both experimental and modeling investigations. The composition of the alloy as determined by Inductively Coupled Plasma Optical Emission Spectrometry (ICP-OES), performed at Anderson Laboratories, Inc. is shown in Table 1.

Table 1: Composition of the Fe-Cr model alloy employed in this study (bal. Fe). The composition was obtained via ICP-OES.

\begin{tabular}{|l|l|l|l|l|l|l|l|}
\hline Element & $\begin{array}{l}\text { Weight } \\
\text { \% }\end{array}$ & Element & $\begin{array}{l}\text { Weight } \\
\text { \% }\end{array}$ & Element & $\begin{array}{l}\text { Weight } \\
\text { \% }\end{array}$ & Element & $\begin{array}{l}\text { Weight } \\
\text { \% }\end{array}$ \\
\hline Si & 0.02 & $\mathbf{C}$ & 0.009 & $\mathbf{C u}$ & $<0.01$ & $\mathbf{A l}$ & 0.014 \\
\hline $\mathbf{S}$ & 0.002 & $\mathbf{C r}$ & 12.02 & Va & $<0.01$ & $\mathbf{T i}$ & $<0.01$ \\
\hline $\mathbf{P}$ & 0.015 & $\mathbf{N i}$ & $<0.01$ & $\mathbf{C o}$ & $<0.01$ & $\mathbf{N}$ & $<0.001$ \\
\hline $\mathbf{M n}$ & $<0.01$ & $\mathbf{M o}$ & $<0.01$ & $\mathbf{N b}$ & $<0.01$ & $\mathbf{O}$ & 0.27 \\
\hline
\end{tabular}

This alloy was chosen because the Fe-Cr binary alloy phase diagram (Figure 3-1) indicates that the $12 \%$ weight chromium alloy would fall just outside the $\gamma$-loop at high temperatures; therefore it will consists of purely ferritic phase without any structural effects from prior high temperature processing.

The model steel was hot rolled at $1150^{\circ} \mathrm{C}$ to a thickness of $0.063 " / 0.070 "$. Samples were cut with Electrical Discharging Machining (EDM) to $20 \times 3 \mathrm{~mm}^{2}$ and $1.5 \mathrm{~mm}$ thick. Before thin film deposition, the samples were mirror polished using standard metallographic techniques, rinsed in water, acetone, ethanol, and then plasma cleaned in a Gentle Asher Chamber, Model A01, for 18 minutes at a power of $50 \mathrm{~W}$. Image of the plasma cleaning system is shown in Figure 3-2 


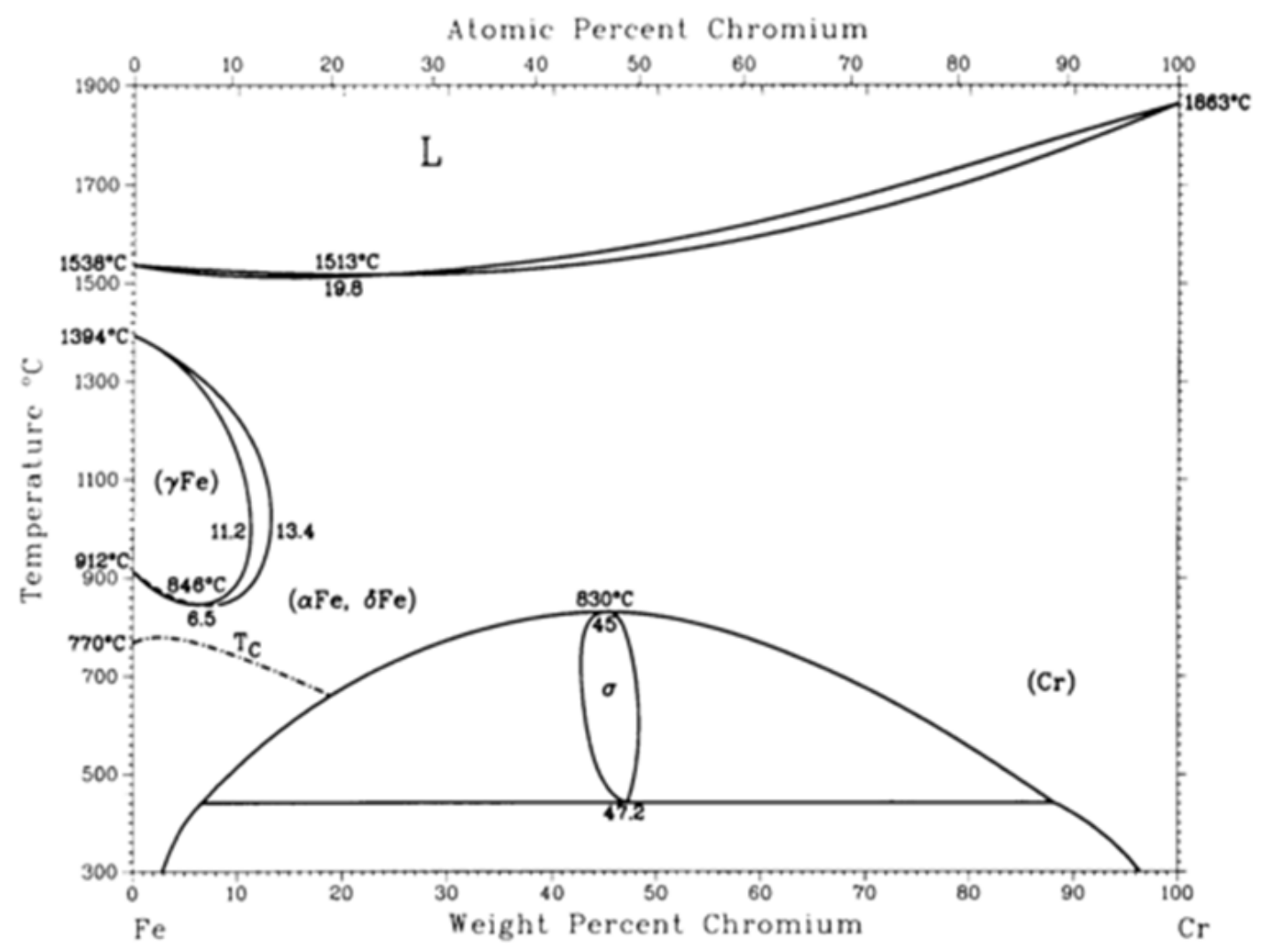

Figure 3-1: Fe-Cr binary phase diagram (from Ref (65)).

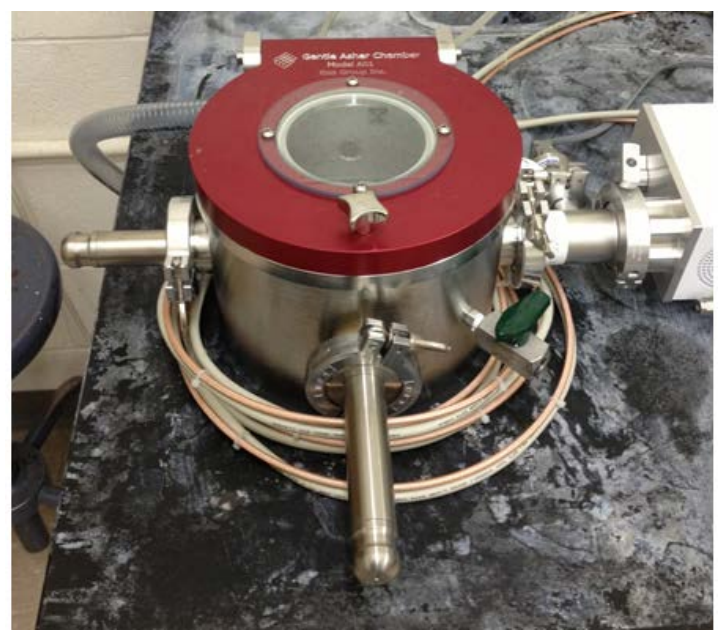

Figure 3-2: View of the chamber of the Gentle Asher Chamber, Model A01, used to plasma clean the Fe-12\% Cr substrates before the deposition of the thin films. 


\subsection{Thin film deposition of titanium and yttrium}

To calibrate the thickness of the deposited films, dummy samples were polished and coated with the metallic films of Ti and Y. The thickness of the films were measured via a cross sectional SEM-image using the Focused Ion Beam (FIB) technique. This process was repeated for each deposition run. A cross sectional view of a titanium thin film coated sample, used to calibrate the deposition system is shown in Figure 3-3. By determining the thickness deposited on the substrate and comparing to the film thickness displayed on the deposition system controller, a calibration factor can be determined.

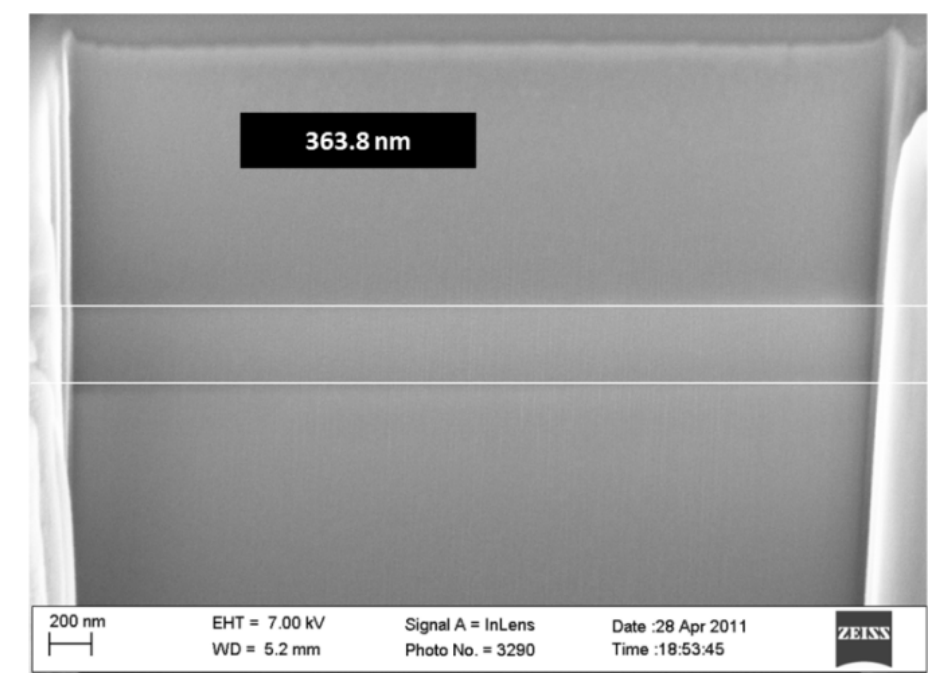

Figure 3-3: Cross sectional SEM view of the titanium as-deposited sample. The titanium film was deposited on a Fe-12\% sample. After milling the sample to obtain a cross sectional view of the coating, the thickness was measured: by knowing the thickness displayed on the deposition system and comparing it with the actual thickness, the real deposition rate was determined.

The sputtering system used to deposit these metallic films was a Denton evaporator (model DV-502) from the Material Science Center facility, at the University of MadisonWisconsin. This system allows for a deposition from a single metallic cathode. Titanium (99.99\% purity) and yttrium (99.95\% purity) 4" diameter, 0.125” thick sputter cathodes were purchased from the company ACI Alloys, Inc. California. The depositions were performed at a base pressure lower than $1 \times 10^{-6}$ Torr. After this base pressure was achieved, the inlet gas (argon) was fed into the system to a pressure of 1.8 mTorr and the deposition was initiated. The targets were pre-sputtered for 2 minutes before the deposition was started, in order to clean the surface of the source from external contamination. During the pre-sputter, a shutter was used to shadow the deposition stage and to protect the surface of the samples. Images of the deposition system are shown in Figure 3-4. 

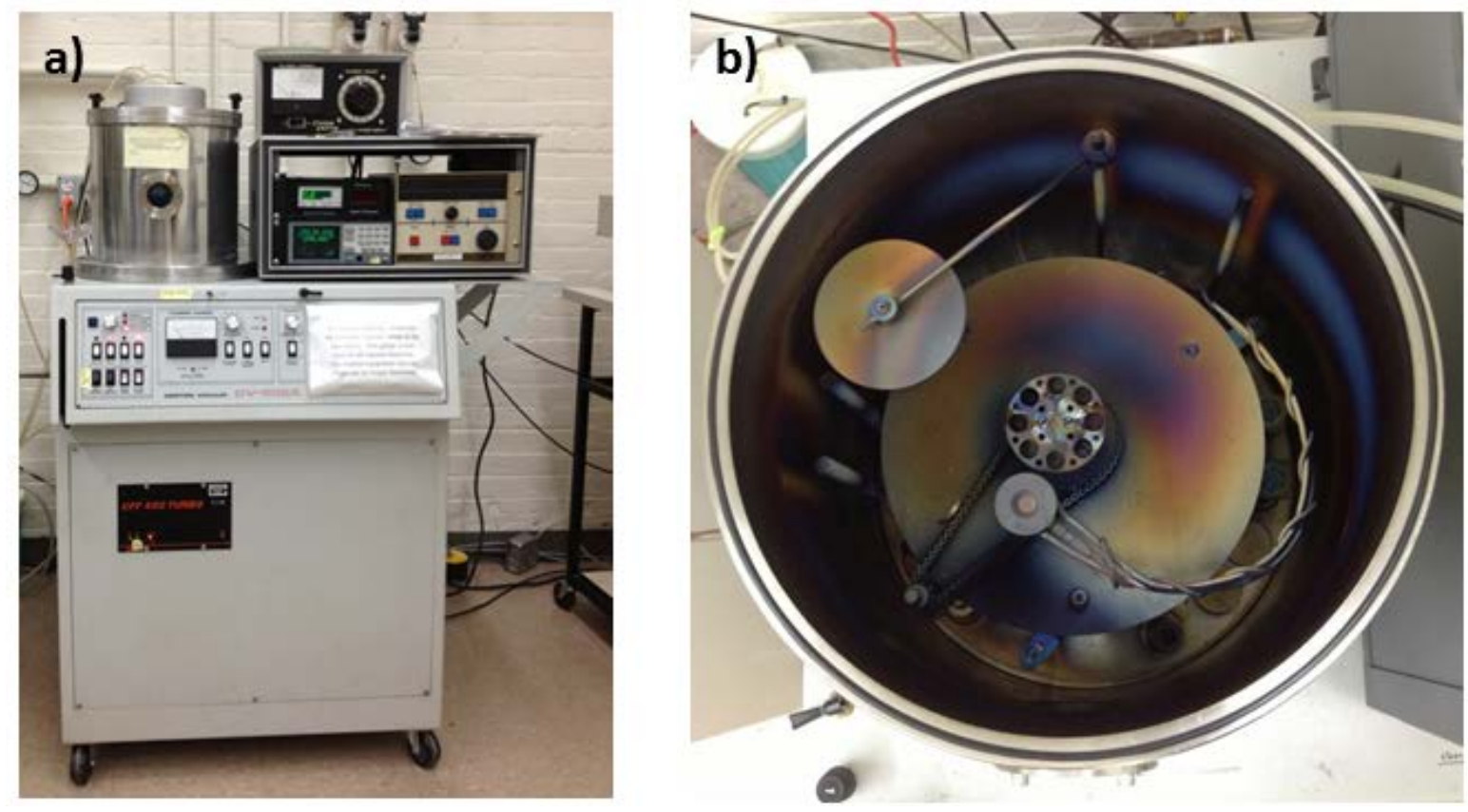

Figure 3-4: Denton evaporator (model DV-502) from the Material Science Center facility a) deposition chamber, power supply and deposition rate controller b) inside of the deposition chamber: quartz crystal thickness control, stage and shutter are visible.

\subsection{Thin film deposition of nonconductive oxides}

Nonconductive insulator targets cannot be sputtered with a DC sputter system, which was employed to deposit metallic Ti and Y thin films. Employing a DC sputtering process with a non-conductive target would lead to charge build on its surface. This in turn would cause the plasma to arc, suppressing the deposition process. This is usually avoided by the employment of an RF sputtering system, where the polarity of the anode-cathode bias is varied at a high rate, which avoids charge build up on the surface of the target.

Oxide film depositions were performed at the Wisconsin Center for Applied Microelectronics (WCAM), at the University of Madison-Wisconsin. This facility has an RF sputtering system which allows the deposition of ceramic insulator films. This system operates at a base pressure better than $2 \times 10^{6}$ Torr and is equipped with four cathodes to allow deposition of multilayers without the need to break the vacuum between successive depositions. Three of the cathode can operate in DC mode, while one can operate in the RF mode. High purity (99\%) $\mathrm{Y}_{2} \mathrm{O}_{3}$ cathode was purchased from the ACI alloys company (the same company that provided the metallic cathodes of yttrium and titanium), while the $\mathrm{TiO}_{2}$ was already available at the WCAM facility. Because of the low thermal conductivity of such oxides, these targets are usually bonded to $\mathrm{Cu}$ backing plates (Figure 3-5). The bonding process usually sets a limit to the power which can be applied to the target and the maximum power limits the maximum deposition rate in the process. 

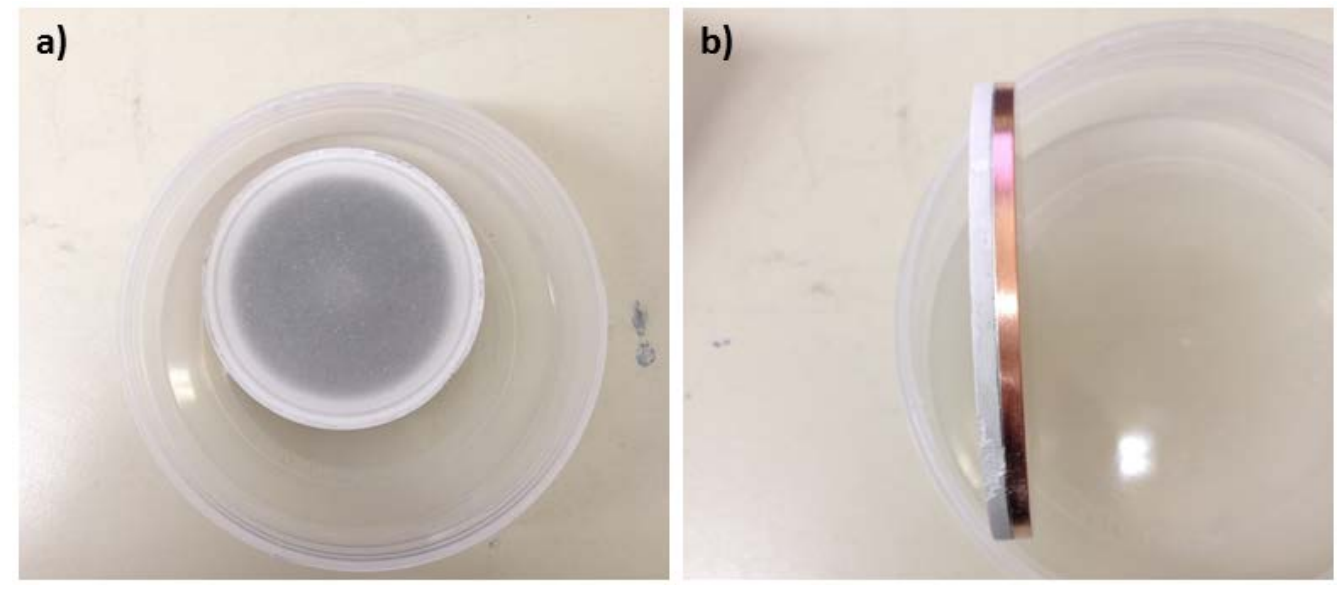

Figure 3-5: $\mathrm{Y}_{2} \mathrm{O}_{3}$ sputter target a) front view b) side view with $\mathrm{Cu}$ backing plate.

To calibrate the system, preliminary depositions were performed on atomically smooth silicon test flats and the film thicknesses were measured with an F-20 optical reflectometer from Filmetrics. Oxide film thicknesses of $\sim 250 \mathrm{~nm}$ were deposited using this system.

Images of the Denton RF sputter located in the WCAM facility are shown in Figure 3-6.
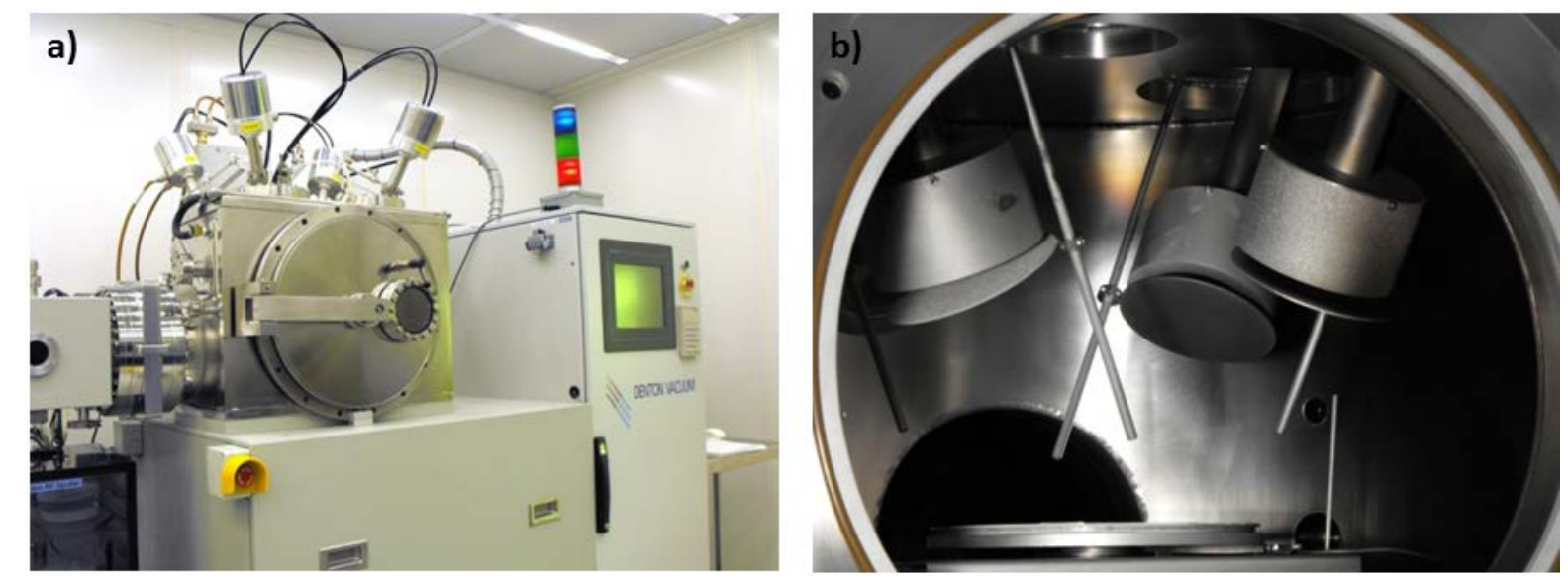

Figure 3-6: WCAM Denton sputterer a) outer view of the deposition chamber b) inner view of the deposition chamber

As can be seen from Figure 3-6, each cathode is covered by a shutter; this allows presputtering the target for a sufficient time in order to clean the surface from of the source from external contaminants and moisture. In the case of the $\mathrm{TiO}_{2}$ deposition, this was done for 40 minutes and in the case of the $\mathrm{Y}_{2} \mathrm{O}_{3}$ this was done for 1 hour. These times were determined during the preliminary depositions, in which it was observed that the deposition rate of these two materials, as determined by the quartz thickness monitor, was stabilizing at constant values after these sputtering times. 


\subsection{Nickel Irradiations}

\subsubsection{SRIM calculations and irradiation experiment design}

After the samples were coated, they were brought to the Pacific Northwest National Laboratory, where the film-substrate systems were irradiated. The ion irradiations were conducted at the Environmental and Molecular Science Laboratory (EMSL) at Pacific Northwest National Laboratory (PNNL), located in Pasco, WA, using $5 \mathrm{MeV} \mathrm{Ni}^{++}$ions. The temperatures for irradiation were $300^{\circ} \mathrm{C}, 500^{\circ} \mathrm{C}$, and $700^{\circ} \mathrm{C}$ in order to cover a broad span of temperatures within 0.2-0.6 $\mathrm{T}_{\mathrm{m}}$ (melting point). The EMSL facility is equipped with a $3 \mathrm{MeV}$ Tandem Pelletron accelerator, a Source of Negative Ions by cesium Sputtering (SNICS), injector and switching magnets. Along the beam line there are several other components: electric steerers to steer the beam, quadrupole magnets to shape and focus it, Faraday cups to read the intensity of the beam and scanners, which allows an effective monitoring of the shape of the beam. Images of the accelerator tank and a quadrupole are shown in Figure 3-7.
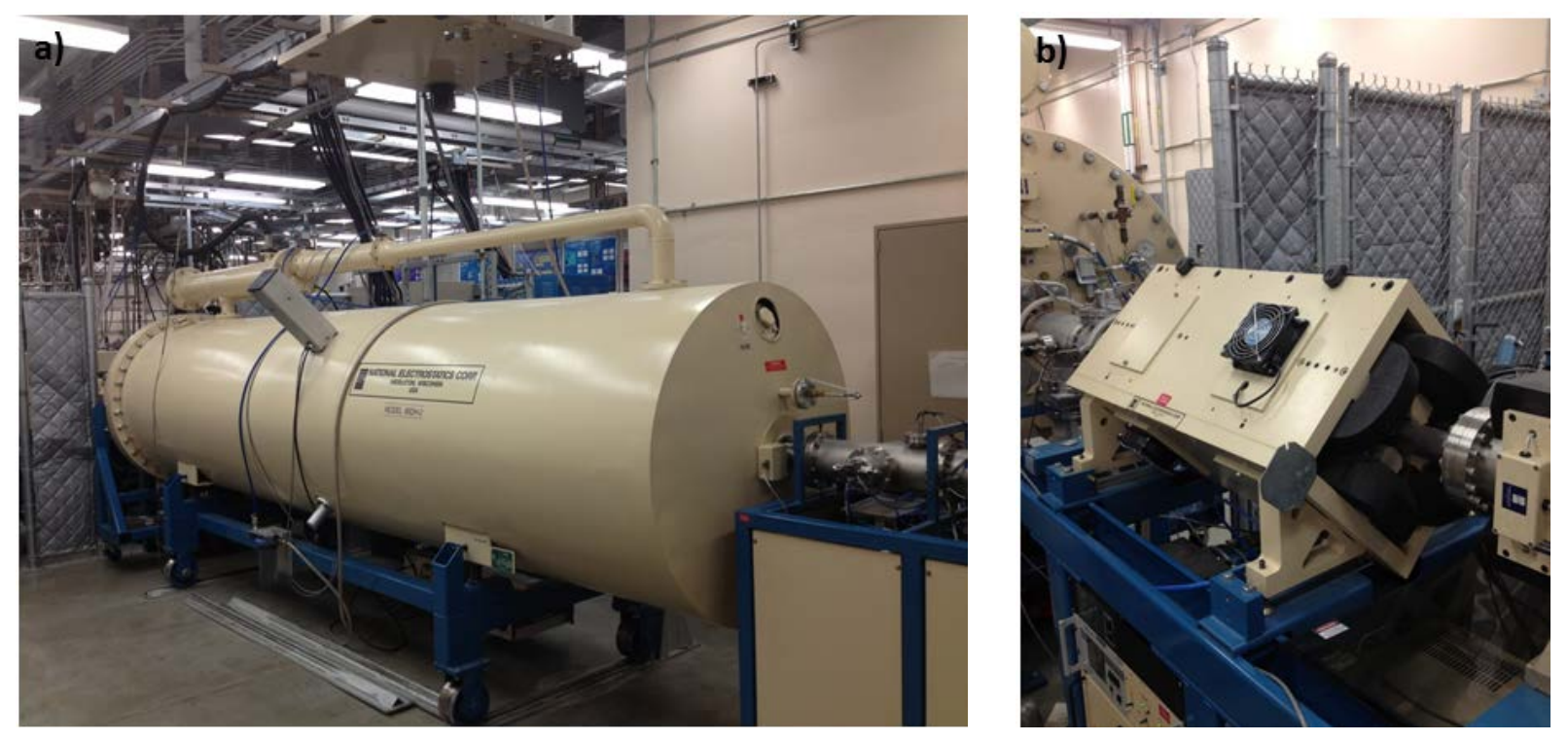

Figure 3-7: EMSL accelerator a) accelerator tank (3MV Tandem Van Der Graaff
accelerator) b) set of quadrupole magnets in the high energy part of the beam.

Negative ions of the irradiating species are produced from a metallic cathode: cesium vapor from a cesium oven enters an enclosed area between a cooled cathode and a heated ionizing surface. Some of the cesium condenses on the front of the cathode and some of it is ionized by the hot surface. The ionized cesium accelerates towards the cathode, sputtering particles from the cathode through the condensed cesium layer. Materials which preferentially sputter neutral or positive particles will pick up electrons as they pass through the condensed cesium layer, producing negative ions (87). Once the negative ions are formed, they are injected into the acceleration tank and accelerated by the positively charged terminal voltage in the middle of the accelerator tank. In the tank, the negative ions are forced to pass through a stripping chamber in which they collide with a gas or a thin foil, losing electrons to them. The 
now positively charged beam experiences a second boost of acceleration from the positive terminal voltage. The energy that can be reached by the ions is governed by the expression:

$$
\mathrm{E}=\mathrm{TV}+\mathrm{Q} \cdot \mathrm{TV}
$$

Equation 3-1

where $E$ is the energy of the ions in $\mathrm{eV}, Q$ is their charge state and $T V$ is the terminal voltage in Volts. With a charge state of +2 , the terminal voltage has to be set up at $1.685 \mathrm{MV}$ to have energy of $5 \mathrm{MeV}$, considering that the species are accelerated out of the cathode with approximately $50 \mathrm{kV}$ energy.

The samples were mounted on a radiation stage that allows temperature control through heating with electron beam and cooling with liquid nitrogen flow in the cooling channel. The first set of the irradiations were conducted by irradiating one sample at the time, for which the ion beam was rastered over an irradiated area of $3 \times 3 \mathrm{~mm}^{2}$ at a current of $200 \mathrm{nA}$. Later on, the irradiations were performed by irradiating two samples at once, for which the ion beam was rastered over an irradiated area of $6 \times 3 \mathrm{~mm}^{2}$ and consequently the ion current was doubled to 400nA: this was done to ensure that whether the samples were irradiated alone or in couples, the irradiation time was going to be kept constant throughout the different irradiations (3 hours and 30 minutes). During the first irradiations, an increase in the amount of current during the implantation was observed and the resulting final times were found to be smaller than the expected. Table 2 shows the irradiation times in minutes for the irradiated samples.

Table 2: Irradiation times (in minutes) for the samples irradiated in this work. The Tiand the $Y$-coated samples were irradiated together at $700^{\circ} \mathrm{C}$; the oxidized samples were all irradiated two-by-two.

\begin{tabular}{|l|c|c|c|}
\hline Coating & $300^{\circ} \mathrm{C}$ & $500^{\circ} \mathrm{C}$ & $700^{\circ} \mathrm{C}$ \\
\hline $\mathrm{Ti}$ & $\sim 180^{\prime}$ & & \\
\hline $\mathrm{Y}$ & $\sim 180^{\prime}$ & $\sim 210^{\prime}$ & $\sim 210^{\prime}$ \\
\hline $\mathrm{TiO}_{2}$ & $\sim 210^{\prime}$ & $\sim 210^{\prime}$ & $\sim 210^{\prime}$ \\
\hline $\mathrm{Y}_{2} \mathrm{O}_{3}$ & $\sim 210^{\prime}$ & $\sim 210^{\prime}$ & $\sim 210^{\prime}$ \\
\hline
\end{tabular}

A rastered beam, rather than defocused beam, was used because the rastered beam can possesses uniform beam intensity. The small size of the beam allowed for having just a fraction of the samples' surface to be hit by the charged particles and the remaining part of it was exposed to the same thermal history, without being irradiated. This part of the samples will be called from now on thermally exposed region. The beam was scanned at a horizontal frequency of $64 \mathrm{~Hz}$ and vertical frequency of $517 \mathrm{~Hz}$. The samples were mounted on the stage as shown in Figure 3-8.

The quoted dpa rate $\left(10^{-3} \mathrm{dpa} / \mathrm{s}\right)$ is based on the average $\mathrm{Ni}$ ion particle flux and the SRIM displacement calculation with full detailed, full cascade calculation and a $40 \mathrm{eV}$ displacement energy for Fe and Cr, 30 for Ti, 25 for Y and 28 for O. 


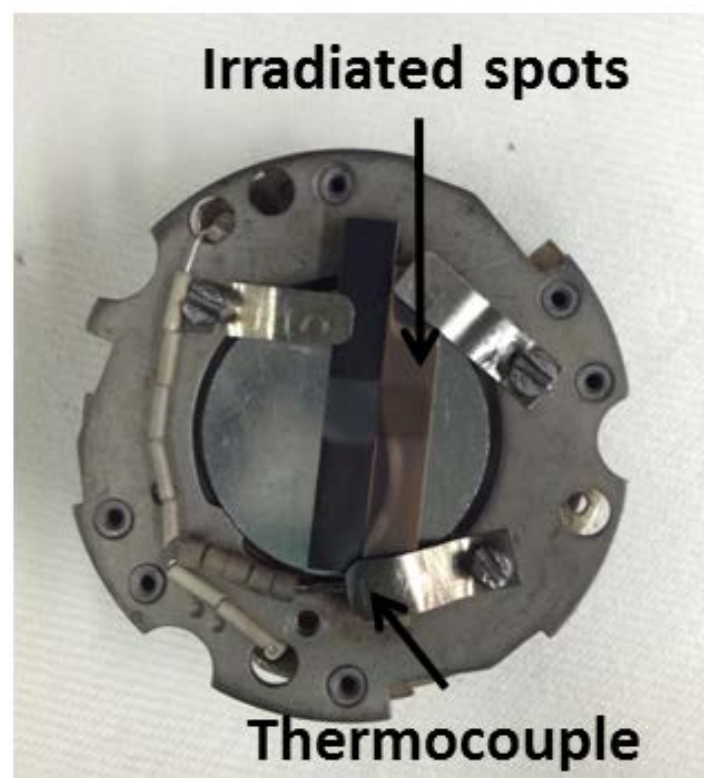

Figure 3-8: Ti and $Y$ samples after irradiation at $700^{\circ} \mathrm{C}$ to a dose of $8.74 \times 10^{6}$ ions $/ \mathrm{cm}^{2}$. The arrows highlight black spots, corresponding to the region hit by the beam and the thermocouple, held on the surface of the sample by a clamp.

To determine the value of the current on the end of the beam line (i.e. on the stage), the accelerator used is equipped with a Faraday cup vertically aligned with the stage. Therefore, every hour, the irradiation was suspended to record the current on the Faraday cup. In cases where the value of the current was different than the one desired, the irradiation times were recalculated accordingly. Currents were always adjusted in order to obtain irradiation time as close as possible to 210 minutes, to ensure that all the samples would be exposed to high temperature for a comparable amount of time and would be damaged at a comparable rate.

The temperature of the samples was monitored using a K-type thermocouple, kept in contact with the surface of the sample, away from the region impinged by the beam (see Figure $3-8$ ). The materials were irradiated to a dose of $8.74 \times 10^{6} \mathrm{ions} / \mathrm{cm}^{2}$ and this corresponds to a damage of approximately $40 \mathrm{dpa}$ in the region of the interface, on the side of the substrate. Figure 3-9 plots the damage profile and implanted ion profile for the $\mathrm{Y}_{2} \mathrm{O}_{3}$ coated samples. Once the irradiation was completed the samples were allowed to cool down on the stage, cooled with $\mathrm{LN}_{2}$ until room temperature. 
a)

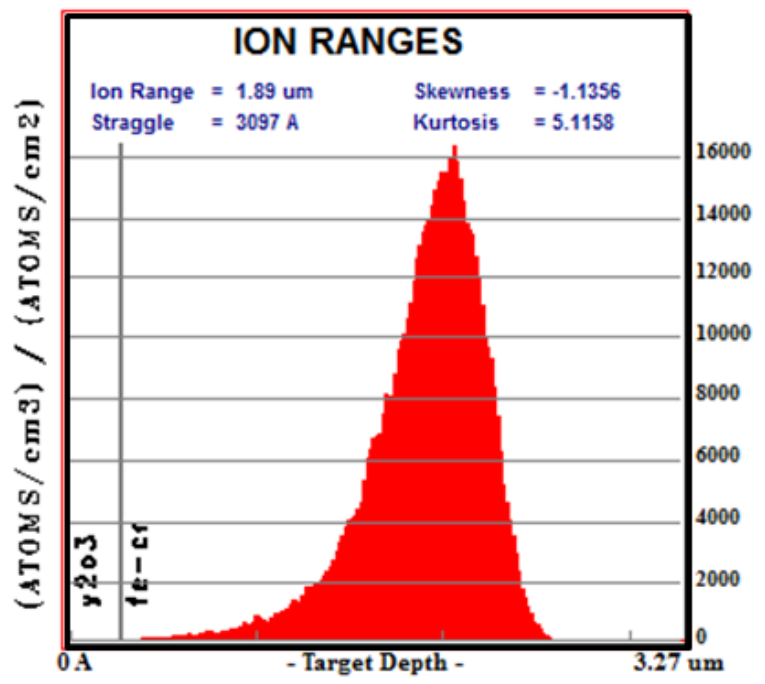

b)

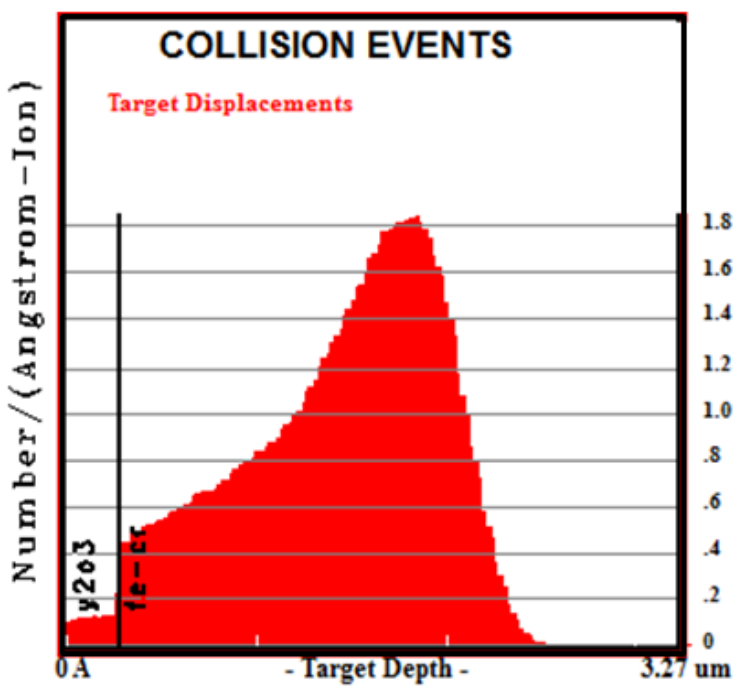

Figure 3-9: SRIM plots comparing the damage profile with the implanted Ni-atom profile for the $\mathrm{Y}_{2} \mathrm{O}_{3}$ coated samples. The energy of the ions was chosen to be $5 \mathrm{MeV}$ in order to minimize the concentration of the atoms implanted near the region of the interface.

TEM samples from the irradiated spots and from regions of the sample which were not exposed to the beam were prepared with FIB lift out techniques.

\subsubsection{TEM and STEM Sample Preparation}

Samples analyzed via TEM and STEM-EDS were prepared using FIB lift out techniques. There are several reasons for which FIB lift out techniques were preferred over other commonly used TEM sample preparation methods. First, the magnetic nature of the material to be analyzed in this case causes significant electron aberrations during TEM and STEM investigation, and the use of FIB lift-out samples eliminates all noticeable aberrations caused by the magnetism of the samples. Moreover the different chemical nature of the substrate and the coatings would cause a different dissolution rate between the two phases during sample preparation with a procedure such as electropolishing. Finally, mechanical techniques were excluded to safeguard the integrity and the adhesion of the coating in the substrate. It must also be noted that FIB lift-outs allow for proper selection of the exact area of the sample to be analyzed. This way samples from the region of the interface between the substrate and the thin films could be lifted out, and samples from different regions of the samples could be chosen accordingly.

Two different FIB instruments were used to prepare the samples: a Zeiss 1540XB Crossbeam FIB/SEM at the University of Madison-Wisconsin and a FEI Quanta 3D FEG DualBeam FIB/SEM housed at the Center for Advanced Energy Studies (CAES) at the Idaho National Laboratory. During the lift-out procedure, a thin cap of platinum was deposited on the surface of the sample, to avoid charging effects and to minimize gallium contamination in the region of interest. Material is removed from both the sides of the platinum cap, shaping a thin lamella ( $2 \mu \mathrm{m}$ thick). Once the lamella is shaped, it can be cut free after one of its sides is 
welded with platinum to a needle. The lamella is then attached to a copper Omniprobe ${ }^{\circledR}$ Lift-Out Grid, which are grids specifically designed to accept the TEM lamellas milled out by FIB systems. These grids have a typical thickness of $25-30 \mu \mathrm{m}$ with a diameter of $3 \mathrm{~mm}$. Different posts are available to weld the lamellas on the grid and they fit standard TEM holders and provide a non-obscured view of the thin sections attached to the posts.

Samples were mounted with a chevron instead or the normal 'flag' mount. The chevron configuration, locks the lamella on both sides of the sample and this prevents the sample from warping or bending during thinning. With some caution, bending can be avoided as well in the flag mounting mode, if the sides and the bottom parts of the lamella are kept thick in order to support the eventual residual stresses of the sample. Figure 3-10 shows a schematic of the two different mounting configurations.
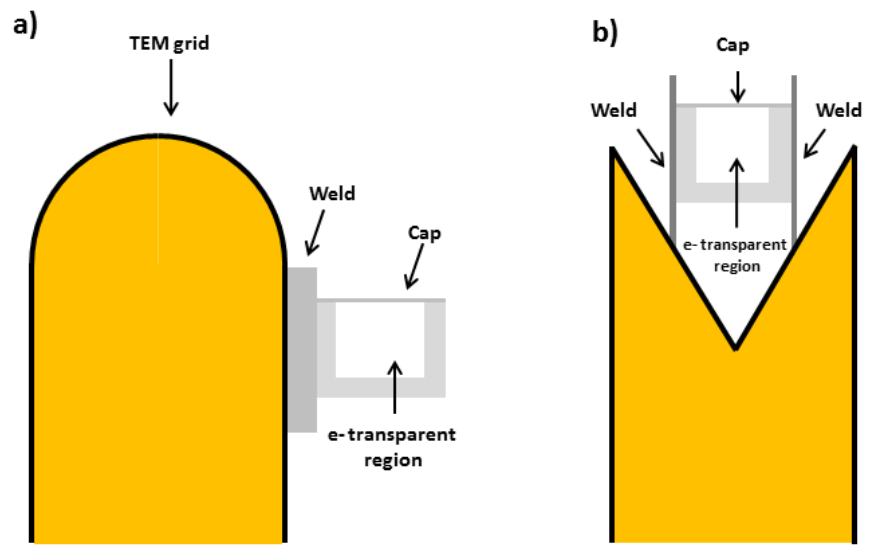

\section{Figure 3-10: Schematic of FIB lift out sample mounting and preparation technique a)flag mounting b)Chevron mounting.}

Once the lamella is attached to the grid, it is thinned out through successive high energy $(30 \mathrm{kV})$ milling steps. At every step the current is reduced in order to minimize the damage caused to the samples by the ion beam. Usually one or two final steps are performed at a lower voltage ( $5 \mathrm{kV}$ and $2 \mathrm{kV}$ ) in order to obtain a flat and clean surface of the TEM sample. In case the samples were not at the desired thickness, they were further thinned with $900 \mathrm{eV}$ argon ions using a 1040 model Nanomill from Fischione. Figure 3-11 shows an electron transparent TEM sample mounted in Chevron position. 


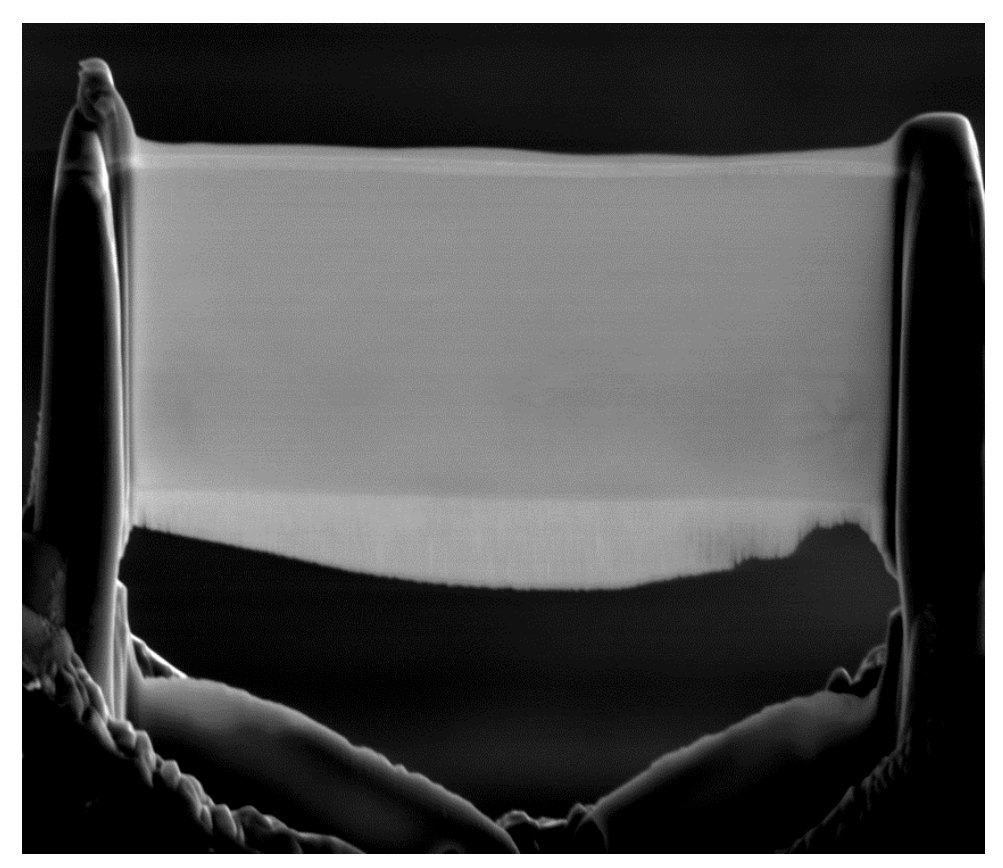

Figure 3-11: SEM view of an electron-transparent yttrium as-deposited sample mounted in the Chevron position.

\subsection{TEM and STEM principles}

\subsubsection{TEM imaging principles}

The Transmission Electron Microscope consists of an electron gun, a set of lenses, detectors/screens, high vacuum column and a specimen holder. Usually a TEM is divided into three components: the illumination system, the objective lens/stage, and the imaging system. In the illumination system, electrons are emitted from the gun and transferred to the specimen though the condenser lenses. The beam can be directed on the sample in parallel, for TEM imaging and selected area diffraction (SAD), or as a convergent beam, used for scanning (STEM) imaging, analysis via x-ray and electron spectrometry, and convergent beam electron diffraction (CBED) (88). At the center of the TEM, the objective lens and the specimen holder/stage system are a critical region where all of the beam-specimen interactions take place. Moreover, the various images and diffraction patterns (DP) are created and subsequently magnified for viewing and recording. The quality of the objective lens determines the quality of all the information of the specimen, thus the objective lens in the most important lens in the TEM. Finally, the imaging system uses several lenses to magnify the image or the DP produced by the objective lens and to focus these on the viewing screen or computer display via a detector, CCD, or TV camera. Figure 3-12 shows the general ray diagram for a conventional TEM 


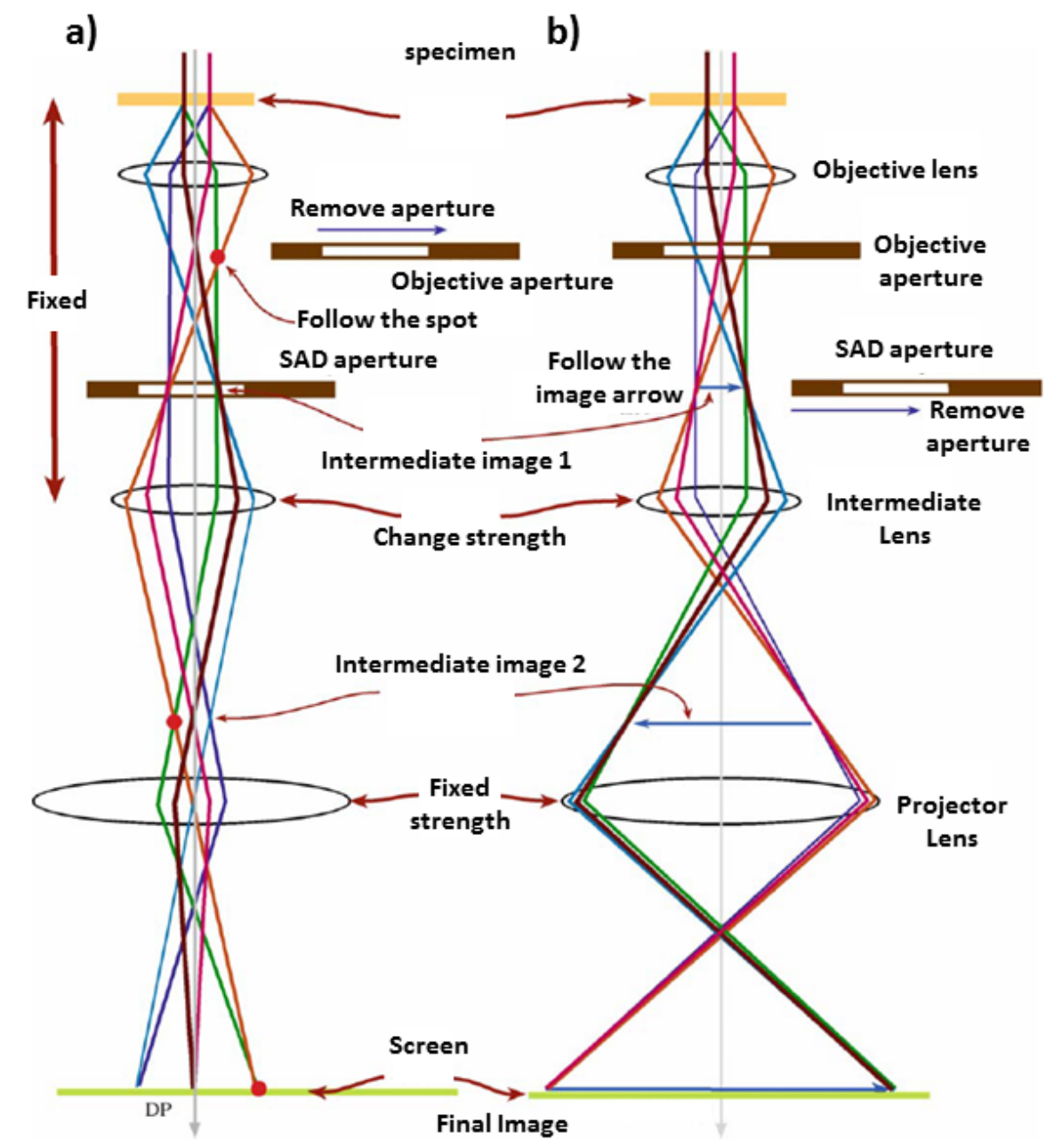

Figure 3-12: The two basic TEM operational principles a) diffraction mode, in which the diffraction pattern projects onto the viewing screen and $b$ ) image mode in which the actual image projects onto the screen.

The image formation mechanism in a TEM instrument is very complicated. Different mechanisms can be used to obtain an image from the specimen and each of these modes can provide different information about the specimen. In this work, the main TEM imaging technique used was High Resolution TEM (HR-TEM). HRTEM is one of the most important aspects of TEM (88). The purpose of this imaging technique is to maximize the useful detail in the image. With current state-of-the-art TEM, sub-nanometer (e.g. atomic) resolution can be routinely achieved.

\subsubsection{STEM imaging principles}

STEM stands for Scanning Transmission Electron Microscopy and implies a transmission electron microscopy performed with a scanned and focused beam (89). In STEM a focused beam is scanned over a thin sample and the intensity of the transmitted electron signals are measured 
with different electron detectors. An image is built up point by point like in a conventional SEM. The essential difference though, is the extremely small size of the beam that is used. If detectors like x-ray or electron energy loss detectors are attached a chemical analysis can be done point by point. Usually STEM microscope uses a field emission electron gun, because only with this can a very narrow, high-current probe (less than $1 \mathrm{~nm}$ in diameter) be delivered to the sample.

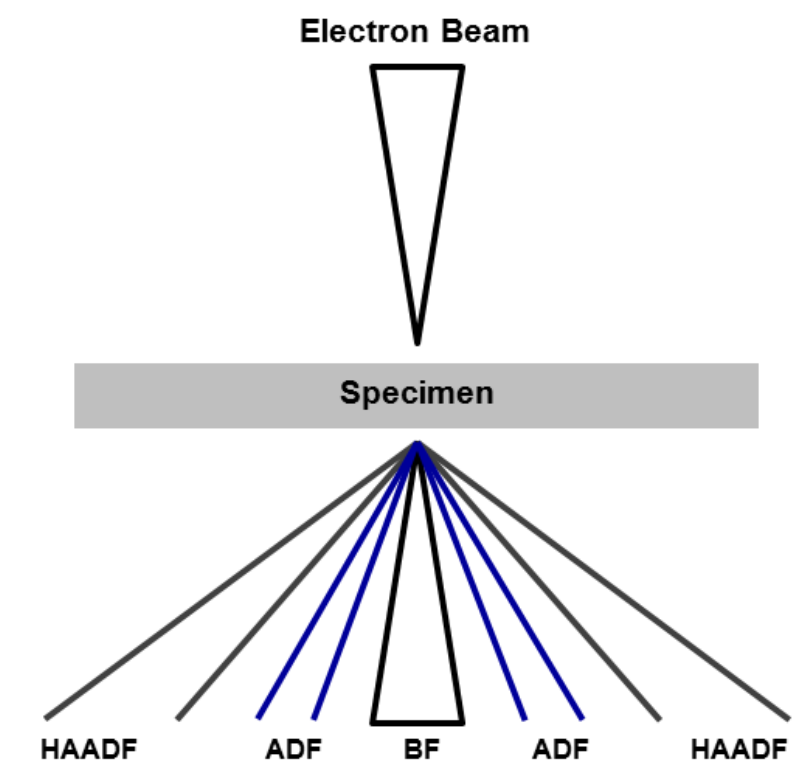

\begin{abstract}
Figure 3-13: Bright Field (BF) detector are axial detectors which collects the electrons that are transmitted right through the sample, Dark Field (DF) detectors collect those electron that are scattered at low angle and High Angle Annular Dark Field (HAADF) detectors collect those electrons that are scattered at high angle from the interaction with the sample.
\end{abstract}

The most important detectors are the Bright Field (BF) detectors, which are axial detectors which collects the electrons that are transmitted right through the sample; Annular Dark Field (ADF) detectors, instead, collect those electron that are scattered at low angle and High Angle Annular Dark Field (HAADF) detectors collect those electrons that are scattered at high angle from the interaction with the sample. Secondary Electron detectors can be added to form an SEM like image. Bright Field images are the images that most resemble conventional TEM images. ADF images show diffraction contrast because the strongly excited diffracted beams are detected. HAADF images show little to no diffraction contrast, since diffracted beams do not contribute to the image formation, leaving only the thermal diffuse scattering to be recorded. HAADF images have atomic number contrast, since heavier elements will scatter electrons at higher angles. These images also do not show contrast reversal with changing focus and specimen thickness. All the STEM investigations were performed using a FEI Titan S-Twin D2080 CEOS Cs corrected (S)TEM operated in STEM mode with an accelerating voltage of 200 $\mathrm{kV}$ housed at the Materials Science Center (MSC) at the University of Madison-Wisconsin. This microscope is equipped with EDAX energy dispersive $\mathrm{x}$-ray spectroscopy (EDS) detector and spectrum image acquisition is handled using FEI's TEM Imaging and Analysis software (TIA). 1-D spectrum profiles were acquired from the region of interest with a ratio pixel to nm equal to 
1, meaning that one point spectra is collected every $1 \mathrm{~nm}$ while the beam is running across the line scan. Dwell time per pixel was chosen in order to obtain approximately a height of 150 counts for the highest peak of the samples, which was the k-edge of iron. Simultaneously ADF signal acquisition was turned on. Drift was minimized to reduce position error over the duration of the scan. Spectrum images were saved in TIA's tagged *.emi format and raw *.ser format. The ADF signal was saved in TIA's tagged *.emi format and in the uncompressed *.tiff format. Quantification of the acquired spectra was performed utilizing the functionality of the TIA software. Quantification was also performed for oxygen. It must be noted that EDS analysis is not entirely accurate for light elements, but, for in the case of ceramic coating, a quantification of this elements was included. 


\section{CHAPTER 4 \\ Experimental Results}

This chapter details the experimental work performed in support of the project objectives. The project workflow generated two samples conditions - irradiated, and unirradiated - for each investigated coating. The results from TEM examination of each coating and condition are presented in the pages that follow.

\subsection{As-deposited metallic thin film examinations}

\subsubsection{Titanium}

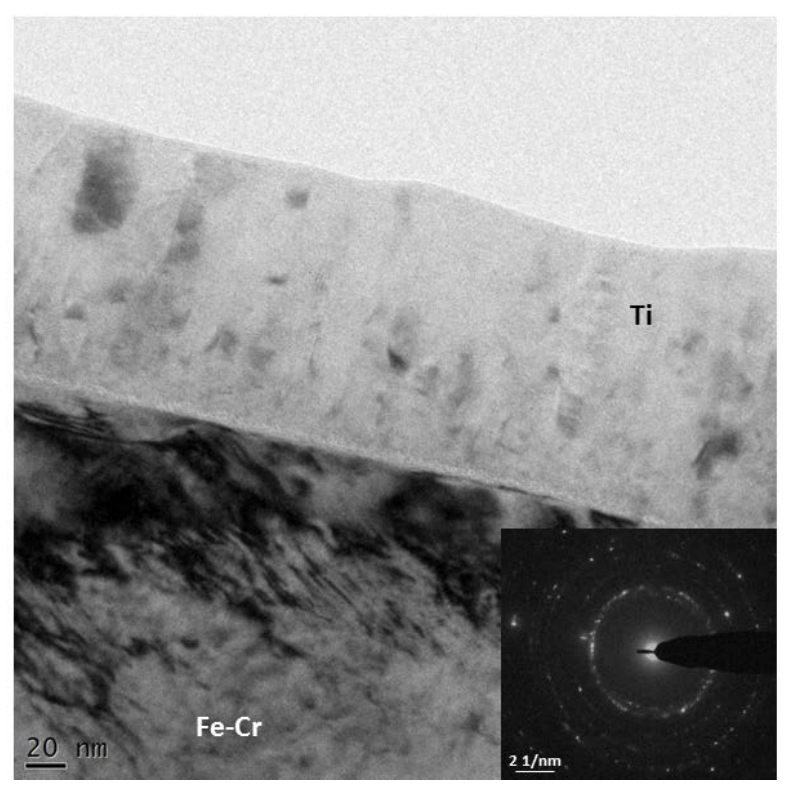

Figure 4-1: BF TEM micrograph of a titanium sample in the as-deposited condition. A columnar structure is observed as is expected due to the use of the sputter deposition technique.

As observed in Figure 4-1, the titanium coating deposits and grows in a columnar structure on the Fe-12\%Cr substrate. Such columnar growth is characteristic of roomtemperature thin film sputter deposition for high melting point materials. Further TEM examination reveals a small $(\sim 7 \mathrm{~nm})$ intermixing region between the substrate and the Ti coating. Figure 4-2 details this region as observed along the [111] body-centered-cubic zone axis. This thin intermixed region likely formed during the first stages of deposition, when the substrate surface is directly exposed to the mildly energetic, incident titanium atoms. 


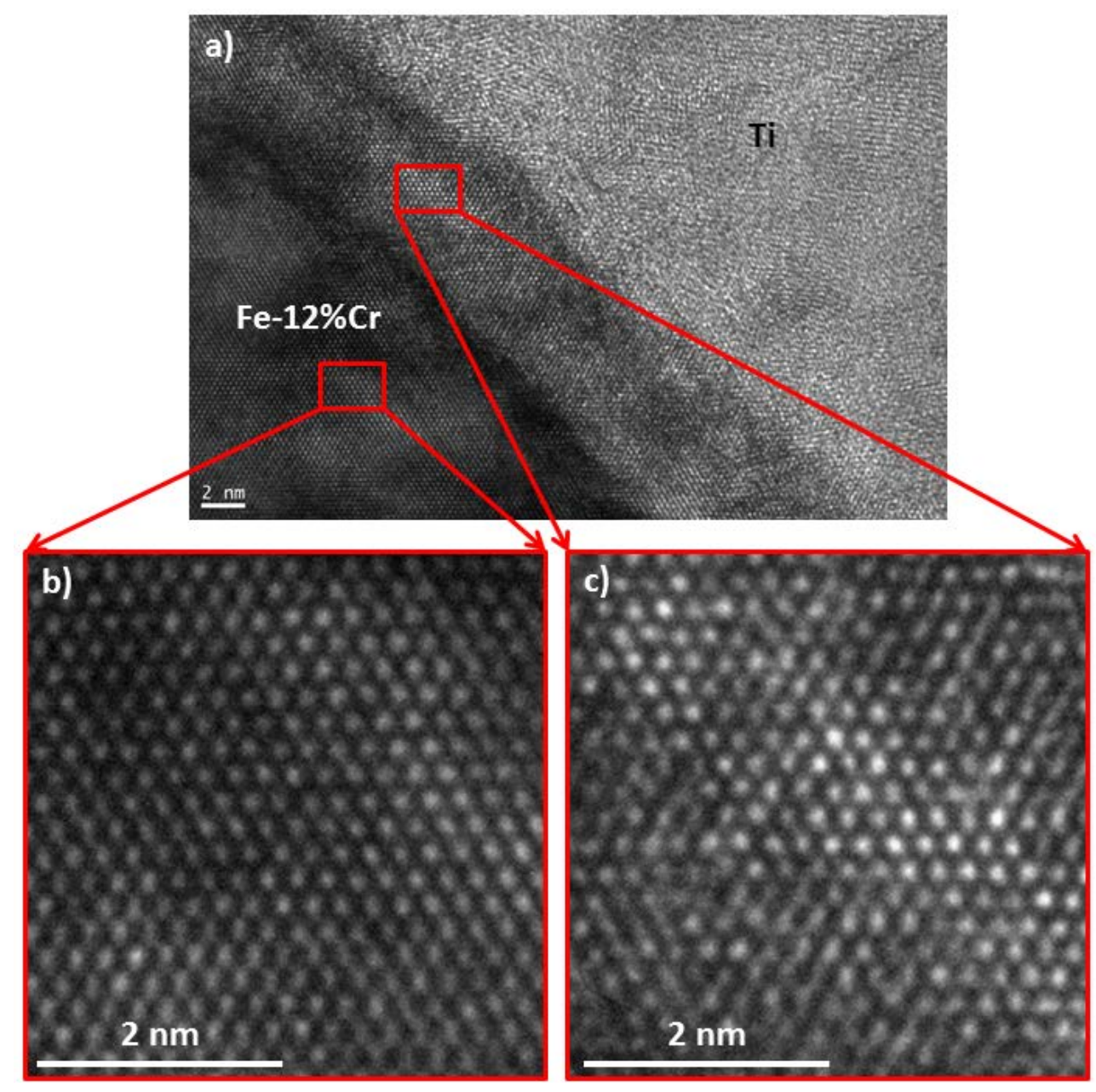

Figure 4-2: a) High Resolution TEM (HRTEM) micrograph along the [111] bcc zone axis of the titanium as-deposited sample, b) magnified substrate, and c) magnified interlayer. Regions in b) and c) have the same atomic structure.

Further characterization of this unirradiated sample included imaging with the ADF detector and performing EDS line scans that traverse the coating, interface, intermixed region, and substrate. In Figure 4-3 (b), EDS composition data clearly indicates that the interface between the substrate and the coating is not perfectly sharp, but rather features a continuously varying composition. 

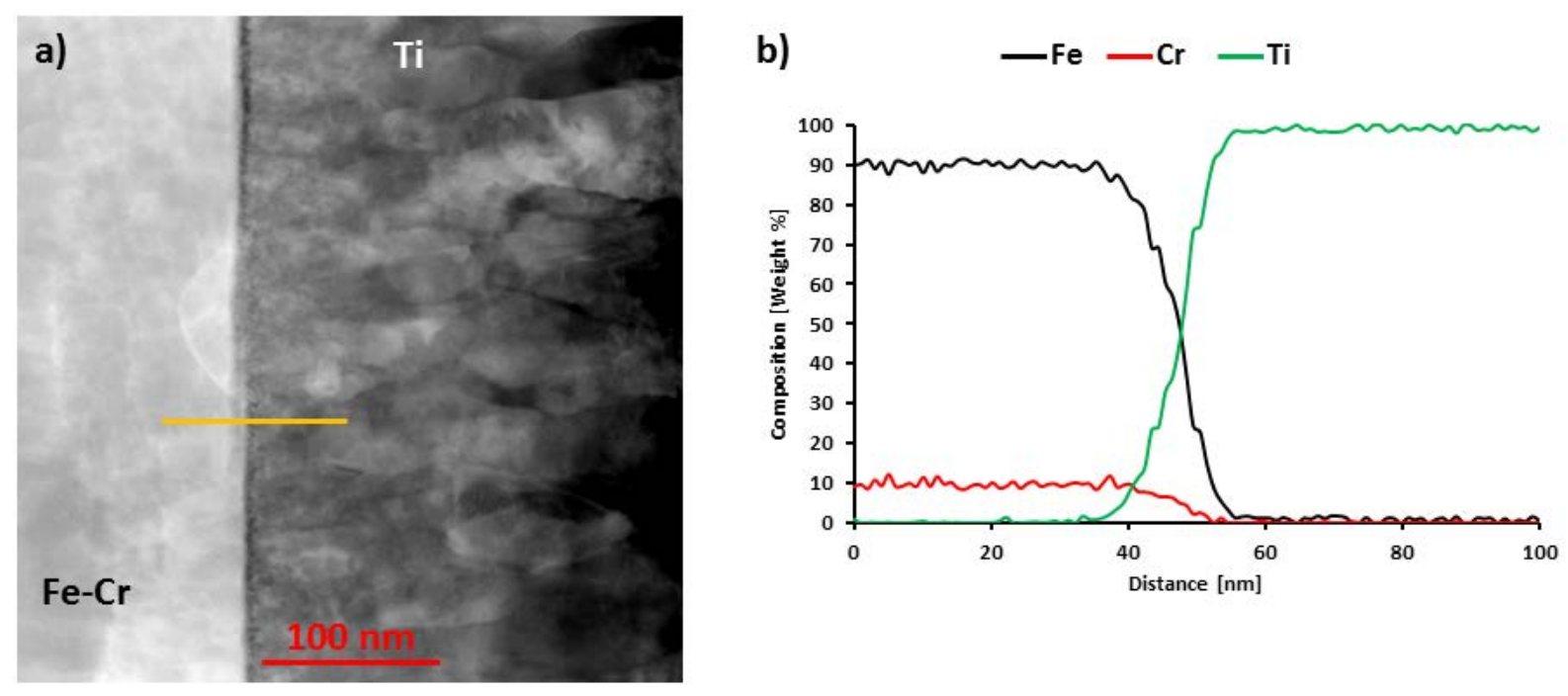

Figure 4-3: Titanium as-deposited sample a) ADF micrograph, and b) the corresponding EDS line profile. A continually varying composition is seen in the interface.

\subsubsection{Yttrium}

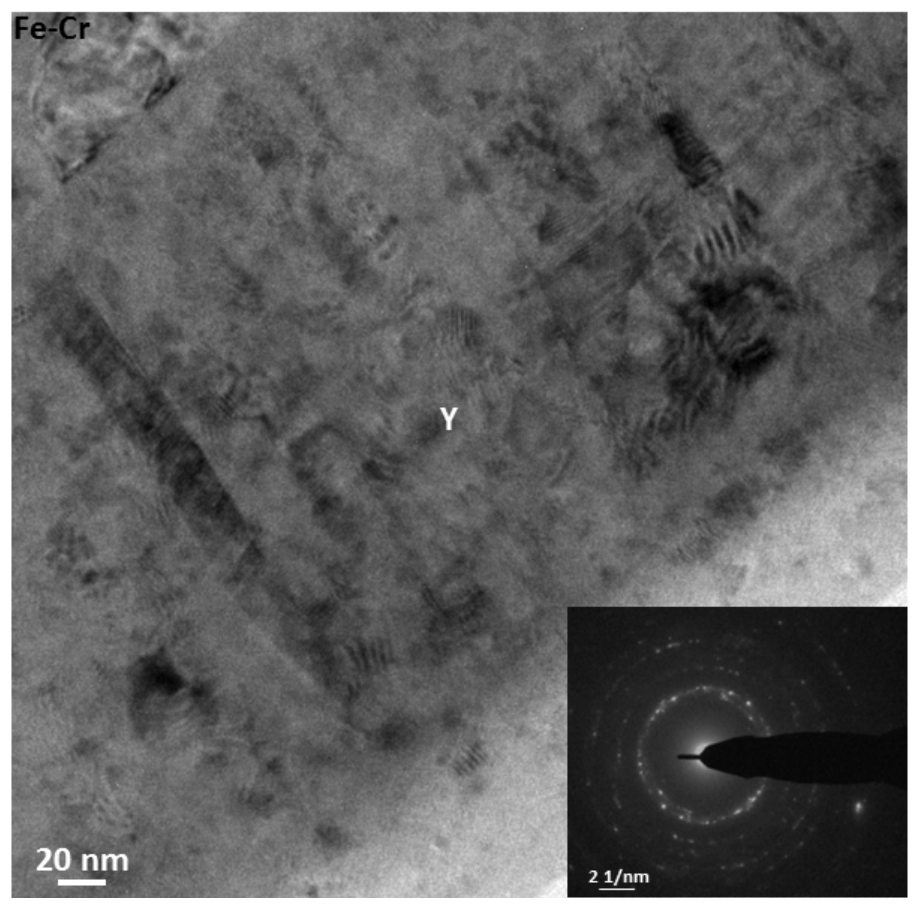

Figure 4-4: BF TEM micrograph of the yttrium as-deposited sample. 
At first glance, comparing the bright field images of Ti (Figure 4-1) and Y (Figure 4-4) thin films following sputter deposition does not yield any cause for interest. As with titanium, sputter deposition of yttrium metal results in columnar growth. However, a closer review of the transition between substrate and coating (top left in Figure 4-4) hints that the interface is more abrupt in for yttrium metal than it is titanium metal. Further imaging in accordance with the established analysis procedure (Figure 4-5) confirms that the interface is notably sharper for sputtered yttrium. This difference and possible mechanisms for its development are discussed in 
Chapter 5.
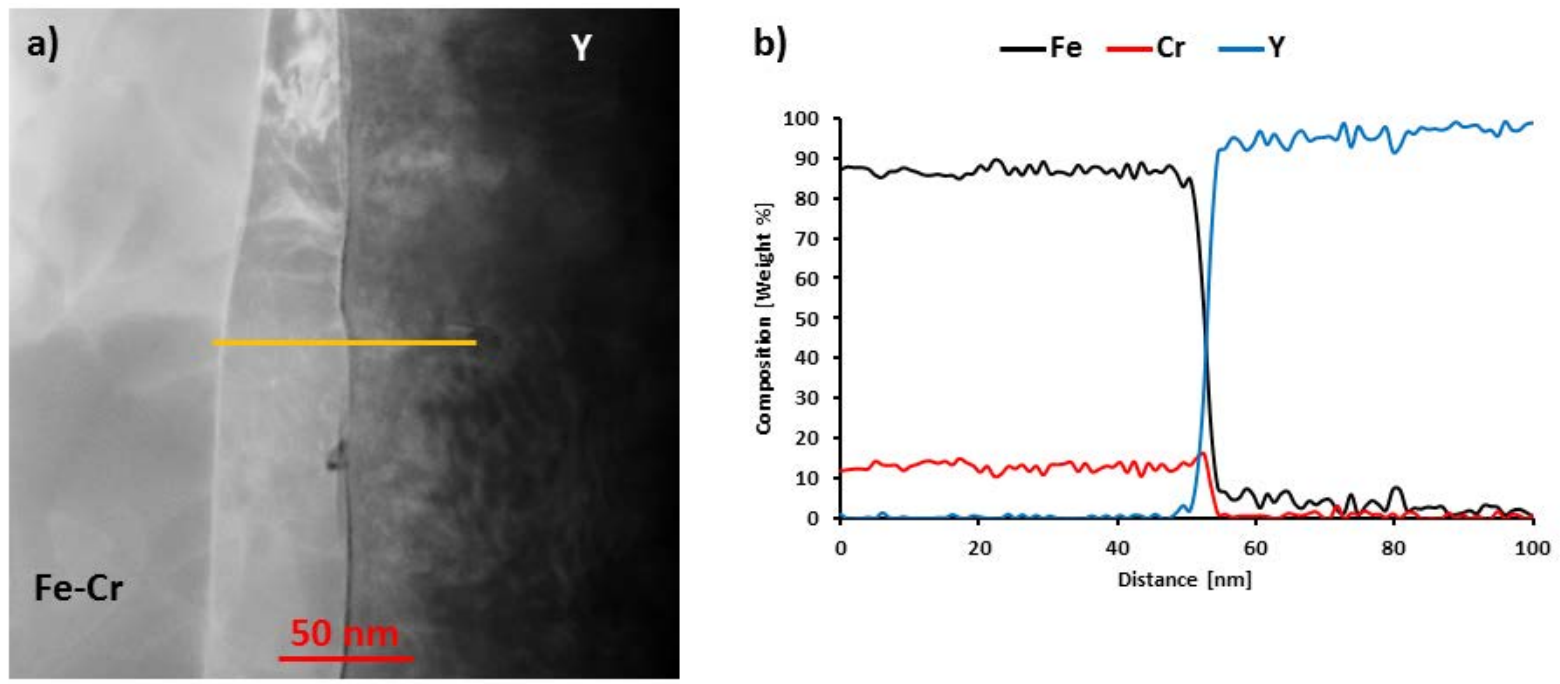

Figure 4-5: Yttrium as-deposited sample a) ADF image, and b) corresponding EDS line profile. A much sharper interface is noted.

\subsection{Irradiated \& Exposed metallic thin film examinations}

As mentioned in Section 3.4.1, the irradiation stage configuration exposes just a small fraction $\left(3 \times 3 \mathrm{~mm}^{2}\right)$ of the samples' surfaces to the beam. Remaining parts of any irradiated sample are not subjected to radiation, but are exposed to the same thermal environment, as the irradiation temperature is largely achieved through stage heating, not beam heating. By preparing TEM samples from both the irradiated and the exposed regions, the effects of temperature and radiation on the microstructural evolution of the system can be separated.

\subsubsection{Titanium and yttrium-coated samples irradiated at $300^{\circ} \mathrm{C}$}

\subsubsection{Titanium-coated sample}

Figure 4-6 shows ADF images and the corresponding EDS elemental analysis line scans of the sample prepared from the titanium-coated specimen exposed and irradiated at $300^{\circ} \mathrm{C}$, respectively. 

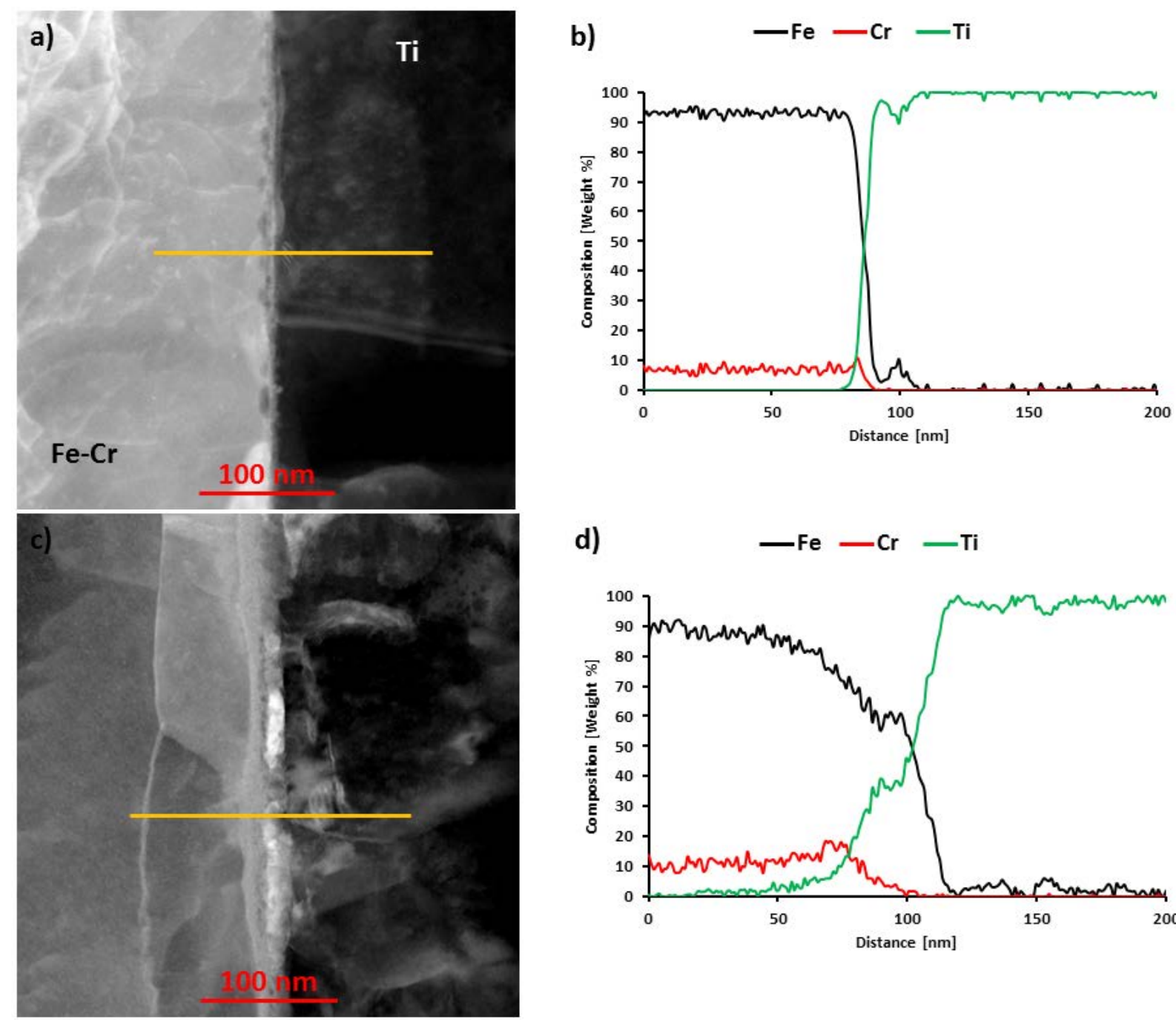

d)

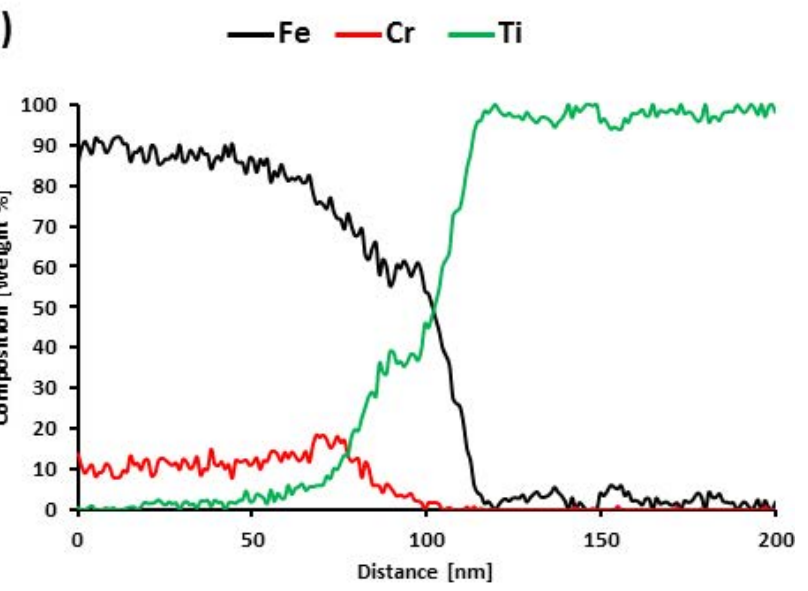

Figure 4-6: ADF images and EDS line scans of the titanium-coated sample (a and b) exposed at $300^{\circ} \mathrm{C}$, and ( $\mathrm{C}$ and d) irradiated at $300^{\circ} \mathrm{C}$. As expected, the exposure for a short period of time (210') at $300^{\circ} \mathrm{C}$ does not cause significant changes in either the morphology or the chemistry of the sample compared to the as-deposited case (Figure 4-3). Irradiation with nickel ions does broaden the interface.

While the morphology of the two samples looks similar, the line scans show the effect of the radiation on the system. The creation of point defect increased the diffusivity of the elements across the interface which in turn increased in extent (passing from $\sim 11 \mathrm{~nm}$ to $\sim 60 \mathrm{~nm}$ ).

\subsubsection{Yttrium-coated sample}

Figure 4-7 shows ADF images and the corresponding EDS elemental analysis line scans of the sample prepared from the yttrium-coated specimen exposed and irradiated at $300^{\circ} \mathrm{C}$. 

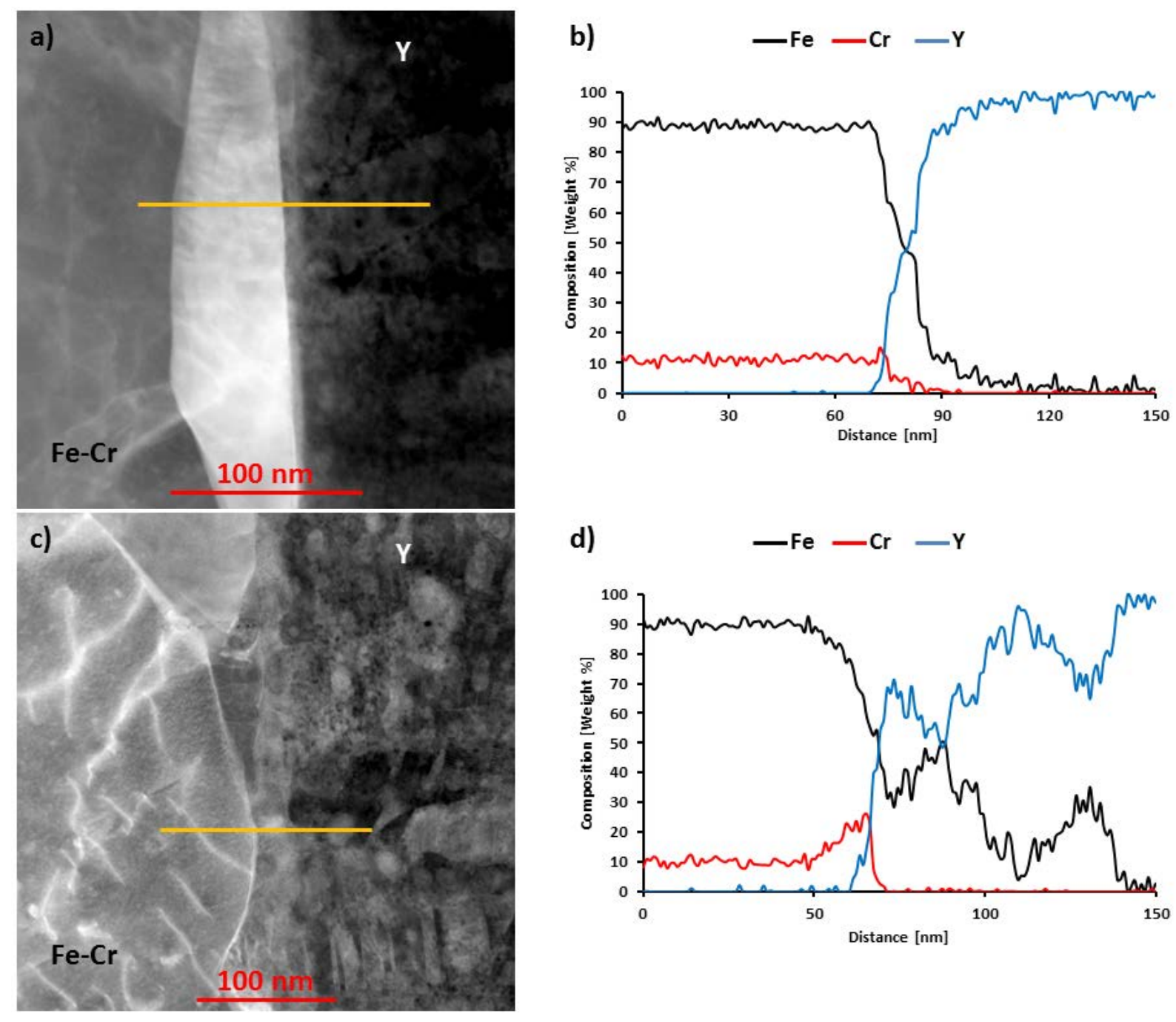

Figure 4-7: ADF images and EDS line scans of the yttrium-coated sample (a and b) exposed at $300^{\circ} \mathrm{C}$, and (c and d) irradiated at $300^{\circ} \mathrm{C}$. Exposure for a short period of time at $300^{\circ} \mathrm{C}$ does not cause significant changes in the sample, while irradiation broadens the interface and helps form small precipitates.

The exposure at $300^{\circ} \mathrm{C}$ did not modify the sample composition or morphology. As expected, the temperature is not high enough to favor appreciable diffusion in the sample. Comparing the two coated samples irradiated under the same condition, a striking difference is observed. In the yttrium-coated sample, small metallic nanoprecipitates enriched in iron and depleted in yttrium are found in the coating region. The determination of the actual nature of these nanoprecipitates is non-trivial and will be discussed in 
Chapter 5.

\subsubsection{Titanium and yttrium-coated samples irradiated at $500^{\circ} \mathrm{C}$}

\subsubsection{Titanium-coated samples}

Figure 4-8 shows ADF images and the corresponding EDS elemental analysis line scans of the sample prepared from the titanium-coated specimen exposed and irradiated at $500^{\circ} \mathrm{C}$, respectively.
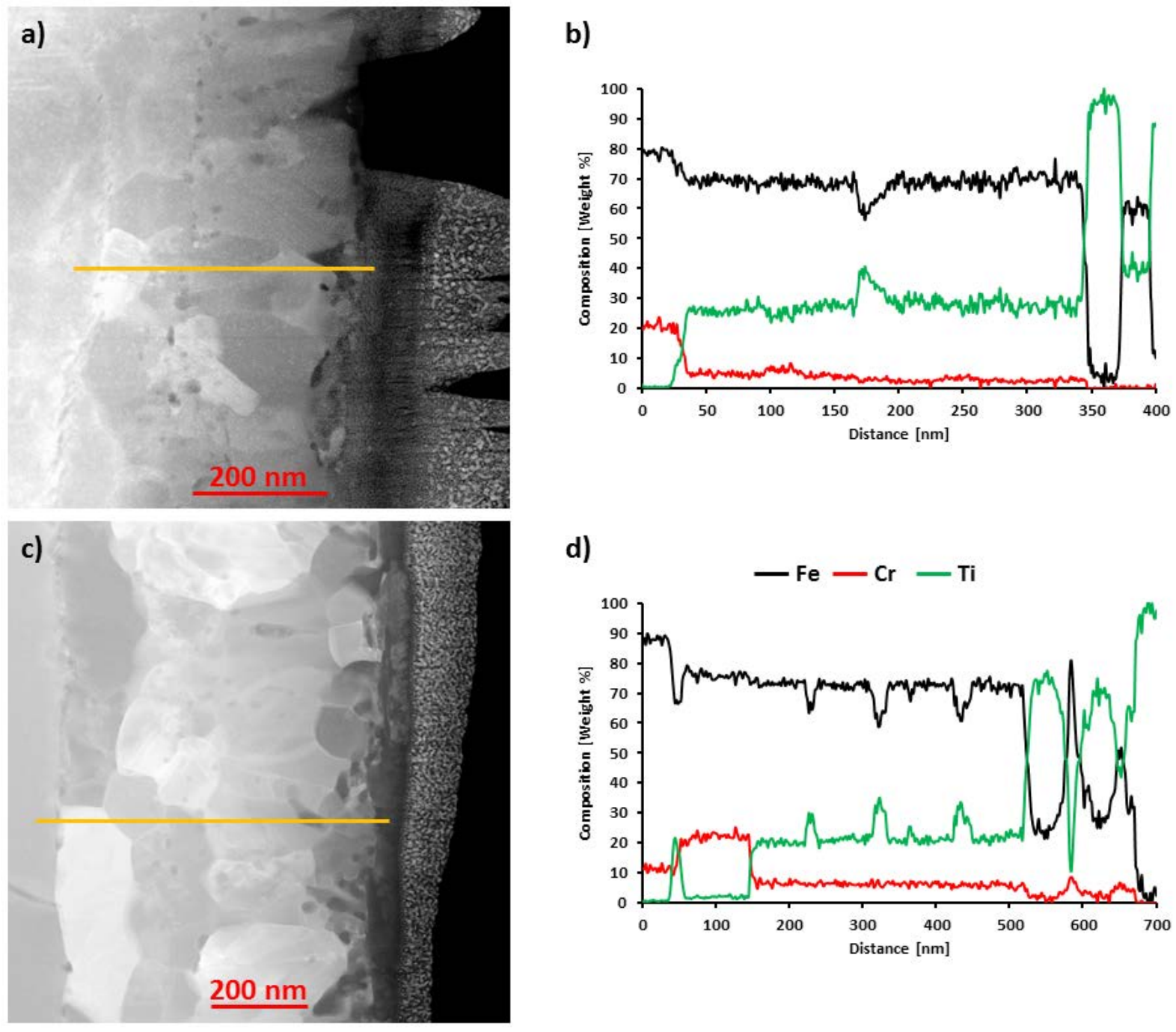

Figure 4-8: ADF images and EDS line scans of the titanium-coated sample (a and b) exposed at $500^{\circ} \mathrm{C}$, and (c and d) irradiated at $500^{\circ} \mathrm{C}$. After exposure at $500^{\circ} \mathrm{C}$ for $210^{\prime}$ a wide intermixed region with intermediate composition forms between the substrate and the coating. Irradiation with nickel ions broadens this region. Titanium-enriched, irondepleted nanoparticles are observed in both conditions. 
As can be seen in Figure 4-8, the temperature effects are now sufficient to cause a significant change in the morphology of the sample, with the formation of a wide intermixed region in which the composition of the sample is constant and intermediate between the substrate and the coating. Irradiation with nickel ions increased the extent of this region from $\sim 340 \mathrm{~nm}$ to $\sim 490 \mathrm{~nm}$. Comparing the two samples it can be noted that the intermixing region is more titanium-enriched (29 weight\%) in the exposed sample than in the irradiated one (21 weight\%, see Figure 4-8) and that a greater concentration of chromium is observed in the latter, despite the similar overall evolution of the system. In this region, nanoparticles enriched in titanium and depleted in iron were found; they correspond to the regions of the EDS line scans in which there is a spike in the iron and in the titanium compositional profiles. In the irradiated sample, the number density of these precipitates is greater as compared to the just thermally exposed sample. The increase in the number of the nanoprecipitates is more pronounced closer to the substrateintermixed region interface and close to the intermixed region-film interface. No obvious correlation was found between their location inside the intermixed region and their composition, except for the ones found very close to the thin film, the substrate-intermixing region interface and the grain boundaries in the $\mathrm{Fe}-12 \% \mathrm{Cr}$ substrate, which are more heavily enriched in titanium, as can be seen in Figure 4-9.
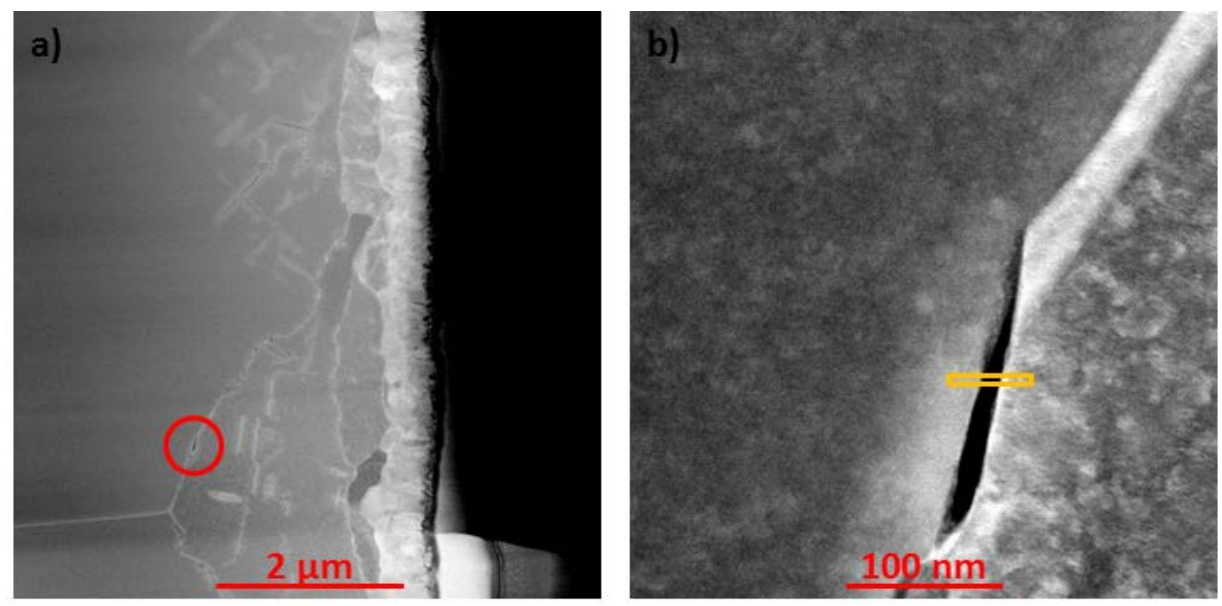

c)

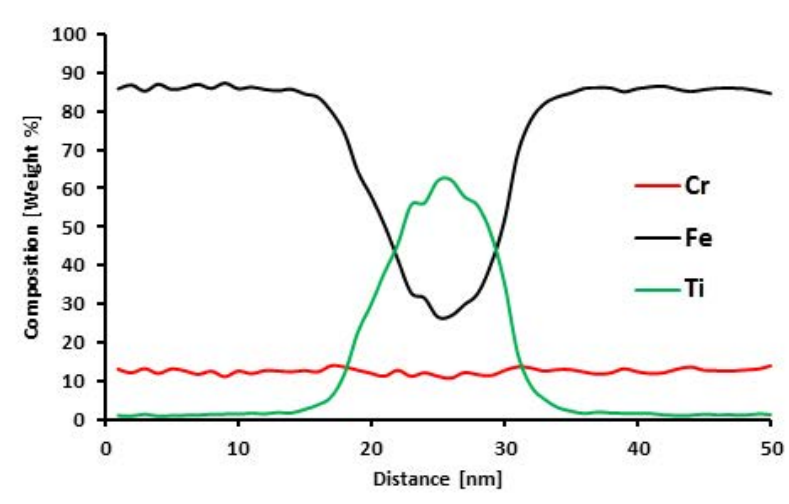

Figure 4-9: a) ADF image of a titanium-enriched nanoprecipitate found along a grain boundary far from the original interface, b) enlargement of the same nanoprecipitate, and c) Composition profile along the nanoprecipitates. The chemical composition was obtained by running 5 different line scans separated by $1 \mathrm{~nm}$ and averaging the results. 
Figure 4-10 shows ADF images and EDS line scans performed on different precipitates of the exposed sample, while Figure 4-11 shows the same analysis performed on the nanoprecipitates of the irradiated samples. As can be seen from Figure 4-10 and Figure 4-11, the three different particles are spatially close and show a very similar composition, and the chromium concentration is unaffected by their presence. Also, the relative enrichment of titanium in the particles of the two different samples is similar. In the thermally exposed samples, the content of titanium increases from 29 weight\% to 49 weight\%, compared to 21 weight\% to 42 weight\% for the irradiated region. It must be noted however that the composition of the particle is dependent upon the thickness of the sample at that point. When the particle is present in the matrix, the EDS analysis will be influenced by the signal coming from the matrix surrounding the particle (see 
Chapter 5 for a detailed discussion).

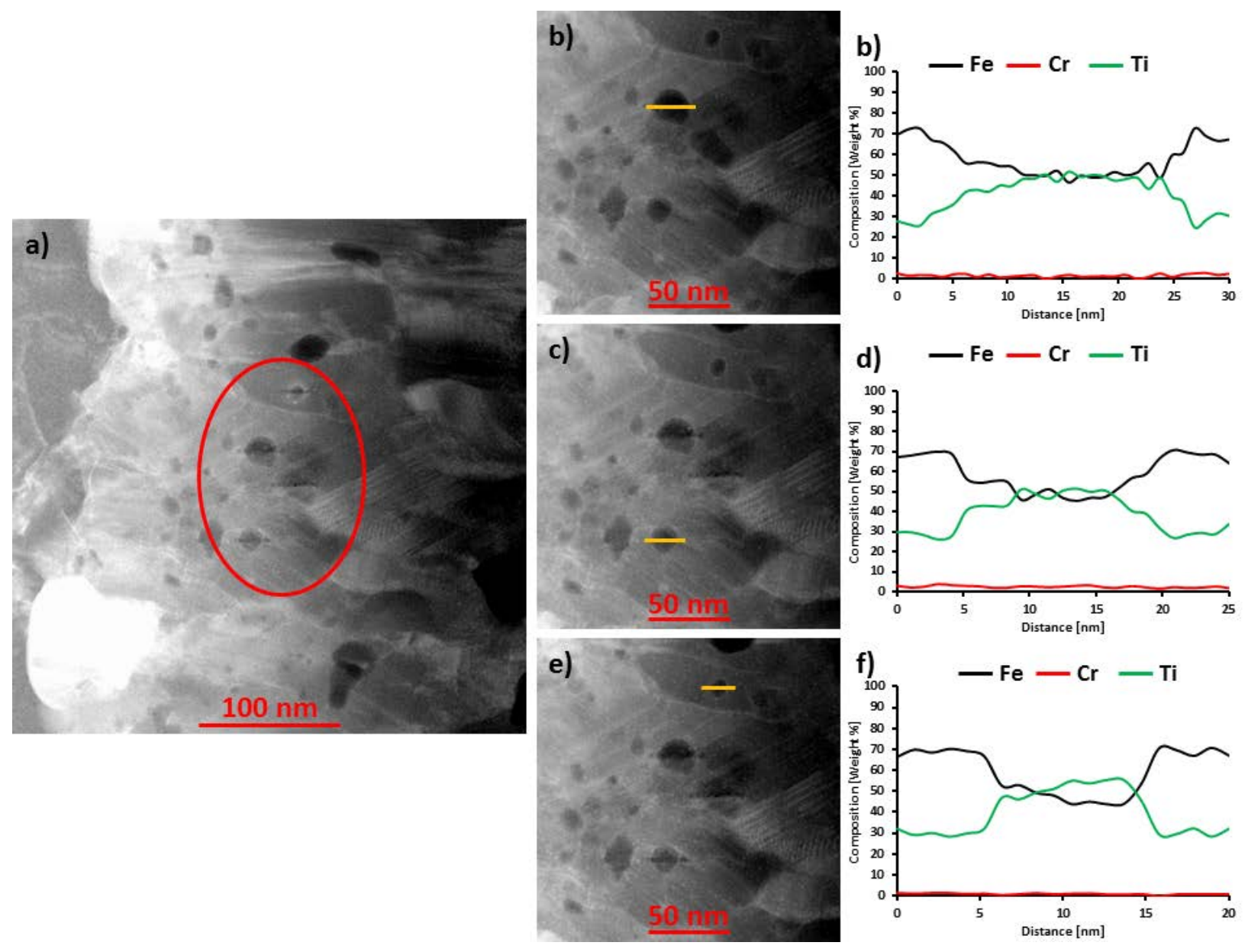

Figure 4-10: ADF images and EDS line scans of three different nanoprecipitates found in the intermixed region of the titanium-coated sample exposed at $500^{\circ} \mathrm{C}$. The precipitates are spatially close to each other and their chemical composition is similar one to the other. 

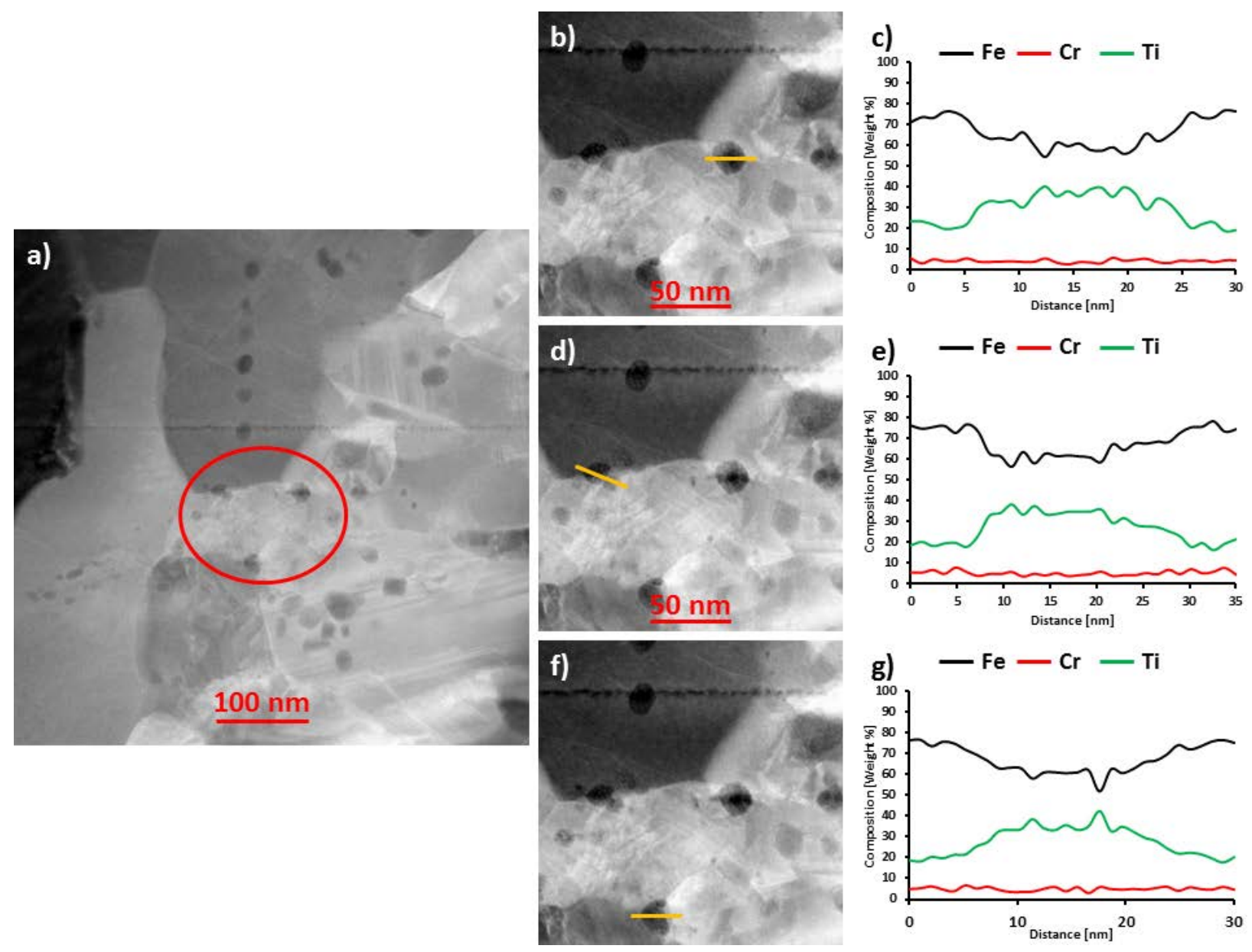

Figure 4-11: ADF images and EDS line scans of three different nanoprecipitates found in the intermixed region of the titanium-coated sample irradiated at $500^{\circ} \mathrm{C}$. The precipitates are spatially close to each other and their chemical composition is similar one to the other.

\subsubsection{Yttrium-coated samples}

Figure 4-12 shows ADF images and the corresponding EDS elemental analysis line scans of the samples prepared from the yttrium-coated specimen exposed and irradiated at $500^{\circ} \mathrm{C}$. Exposure at $500^{\circ} \mathrm{C}$ caused the formation of a wide intermixed region. Irradiation with nickel ions increased the extent of this region from $\sim 360 \mathrm{~nm}$ to $\sim 430 \mathrm{~nm}$. While in the exposed samples the intermixed region has an almost constant composition, in the irradiated samples it has regions which are predominantly enriched in yttrium alternated to regions predominantly enriched in iron. 

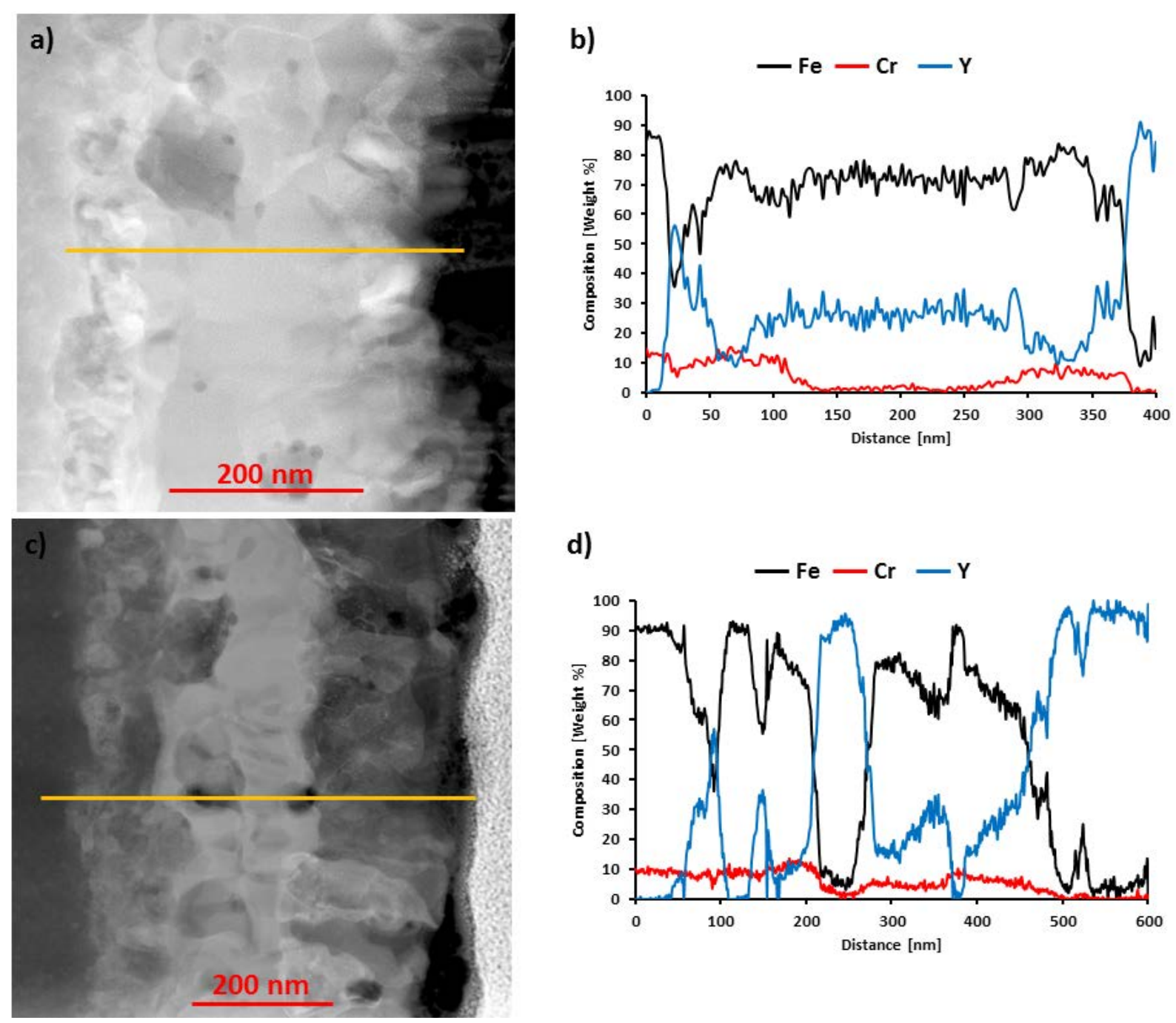

Figure 4-12: ADF images and EDS line scans of the yttrium-coated sample (a and b) exposed at $500^{\circ} \mathrm{C}$, and (C and d) irradiated at $500^{\circ} \mathrm{C}$. Exposure at $500^{\circ} \mathrm{C}$ for $210^{\prime}$ caused the formation of a wide intermixed region with almost constant composition. Irradiation causes the formation of regions more enriched in yttrium alternating to regions more enriched in iron.

In the intermixed region, for both the samples, small precipitates are detected and were analyzed by EDS, but no consistent composition was observed. Large-sized yttrium precipitates were also observed at the substrate-intermixed zone interface, on the Fe-12\%Cr alloy side (Figure 4-13). As can be seen from the EDS elemental line profile, in both the samples these precipitates are heavily enriched in yttrium and depleted in iron. 

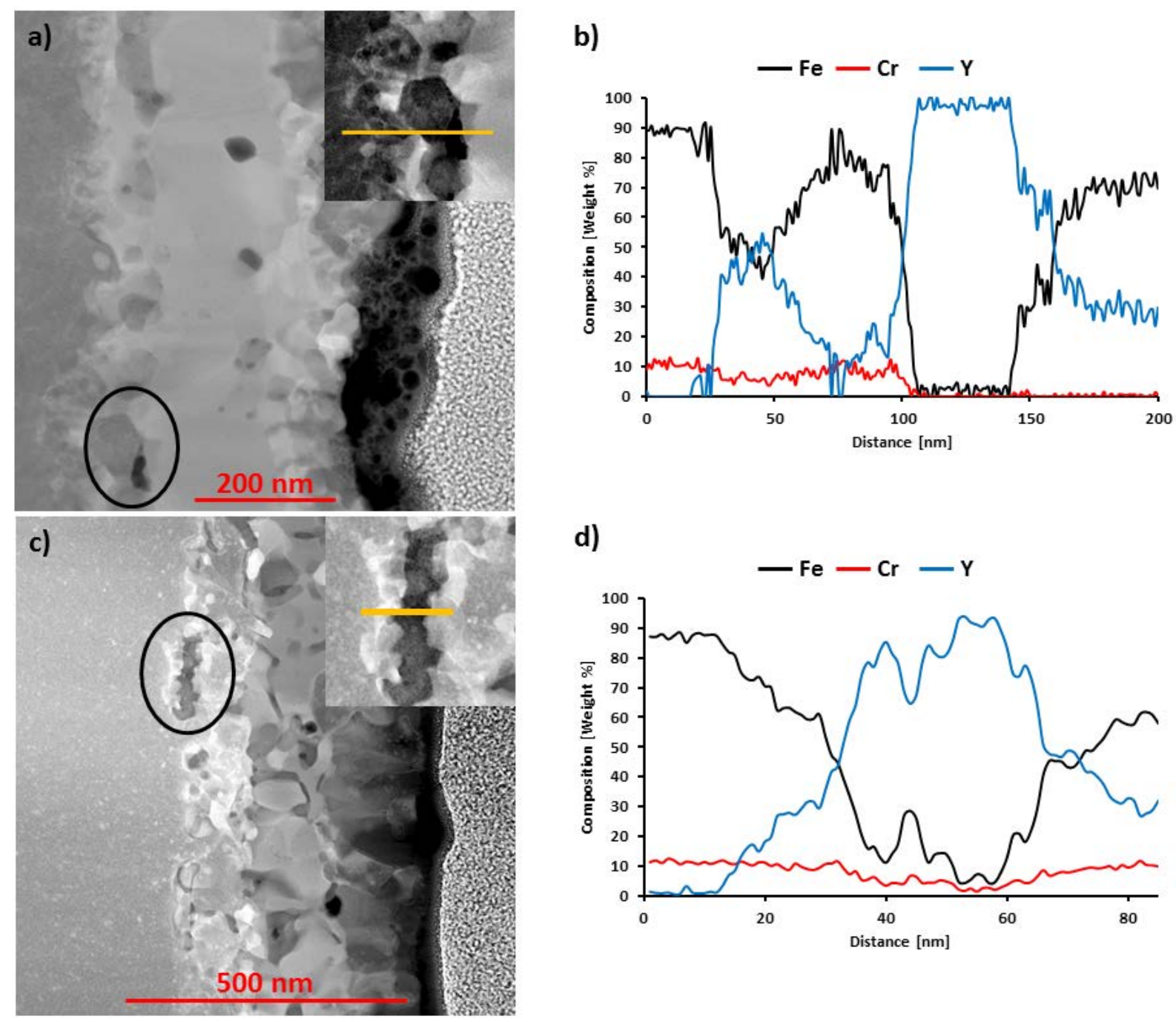

d)

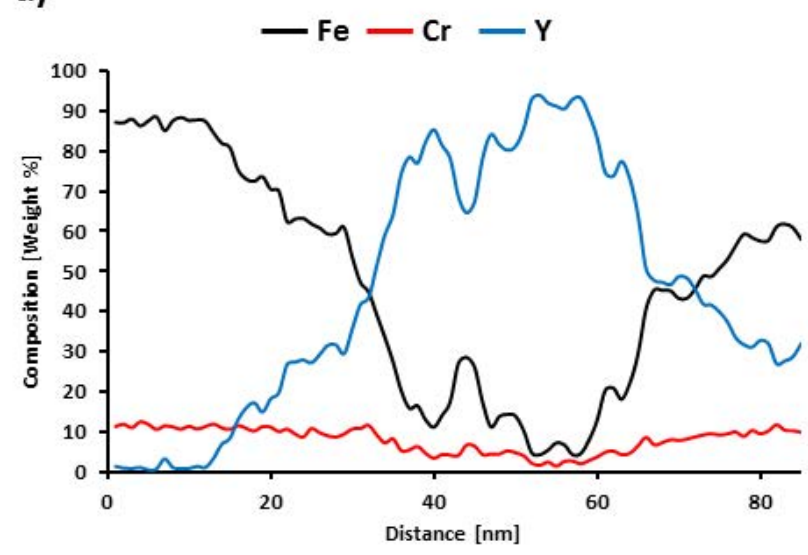

Figure 4-13: ADF images and EDS line scans of the yttrium rich inclusions found at the substrate-intermixed zone interface (a and b) exposed, (c and d) irradiated. In the circled insets of a) and c), the line scans alignment is shown.

\subsubsection{Titanium and yttrium-coated samples irradiated at $700^{\circ} \mathrm{C}$}

\subsubsection{Titanium-coated sample}

Figure 4-14 shows ADF images and the corresponding EDS line scans of the $700^{\circ} \mathrm{C}$ exposed and irradiated titanium-coated specimen samples, respectively. The images indicate the two samples share a similar morphology, which is not expected when considering the thermal and irradiation responses observed during the $500^{\circ} \mathrm{C}$ experiment. In Figure 4-15, the samples' post-treatment morphology is further evaluated with trans-interface EDS line scans to determine the composition. 

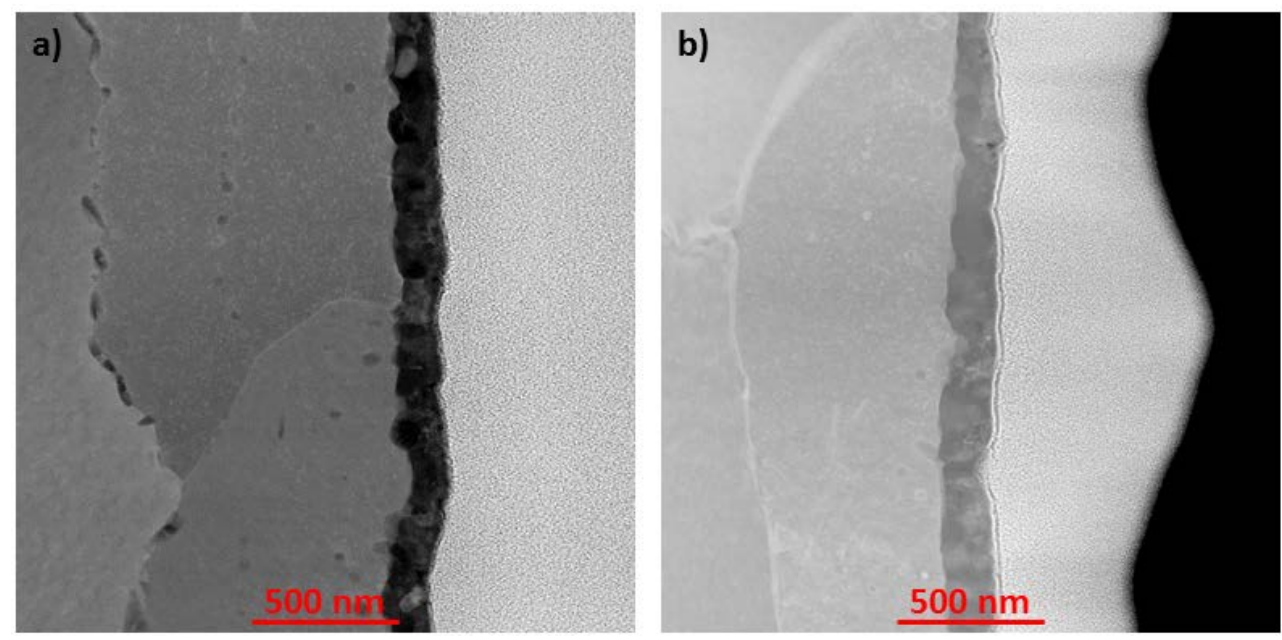

Figure 4-14: Low-magnification ADF images of the titanium-coated sample do not show significant differences between exposed $(a, b)$ and irradiated $(c, d)$ conditions.

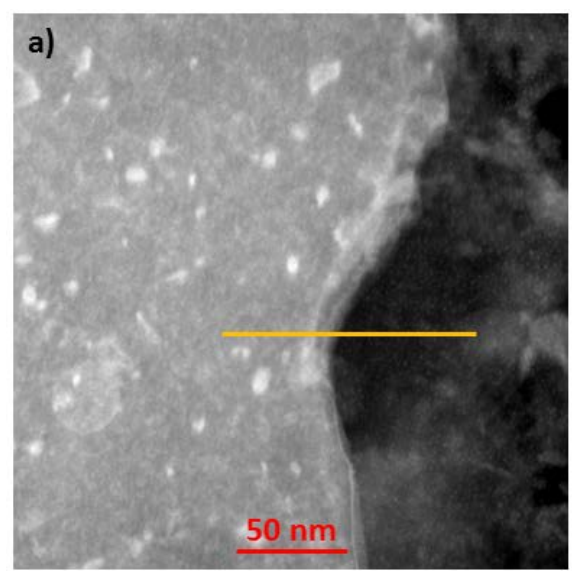

b)

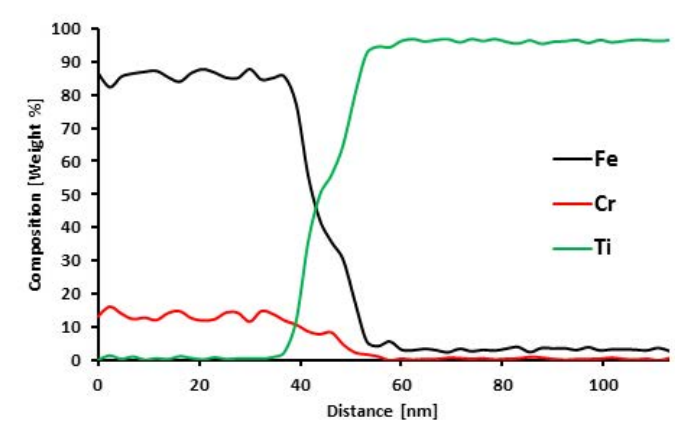

c)

d)

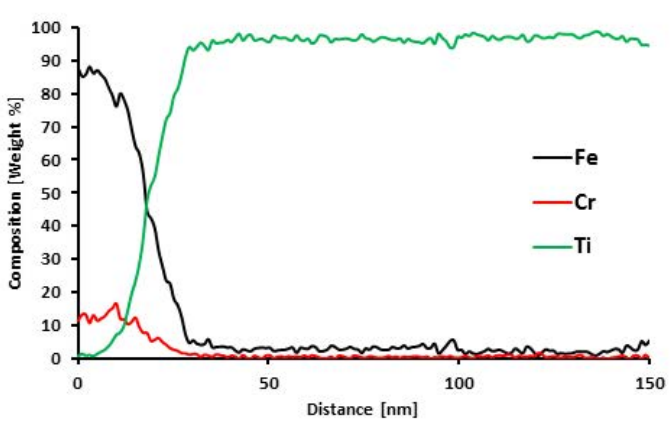

Figure 4-15: ADF images and EDS line scans of the titanium-coated sample. (a and b) exposed at $700^{\circ} \mathrm{C}$, and (c and d) irradiated at $700^{\circ} \mathrm{C}$. Near-coating nanoprecipitates are observed without the presence of an intermixed region. 
As can be seen from Figure 4-15, the evolution of the system after exposure at $700^{\circ} \mathrm{C}$ and after irradiation does not follow the trends observed in $300^{\circ} \mathrm{C}$ and $500^{\circ} \mathrm{C}$ experiments. The original coating appears intact, though thinner than the as-deposited thickness. No intermixed region is observed in these samples, and EDS line scans (Figure 4-15 (b), (d)) indicate the substrate-thin film interface is sharp. The possible sources of these observations are discussed in 
Chapter 5. Few nanoprecipitates were found in the system and the analysis carried on them is shown in Figure 4-16.
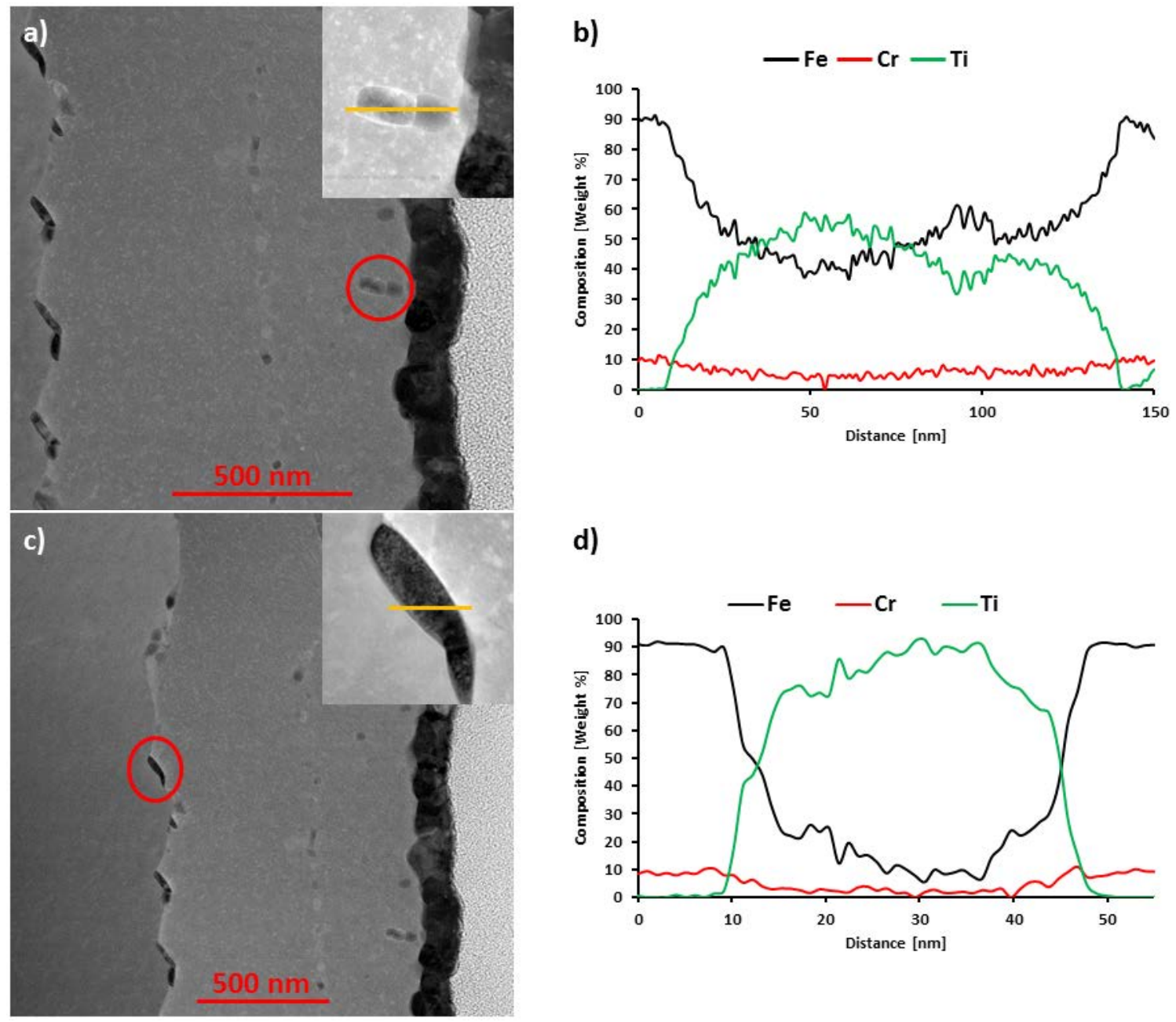

d)

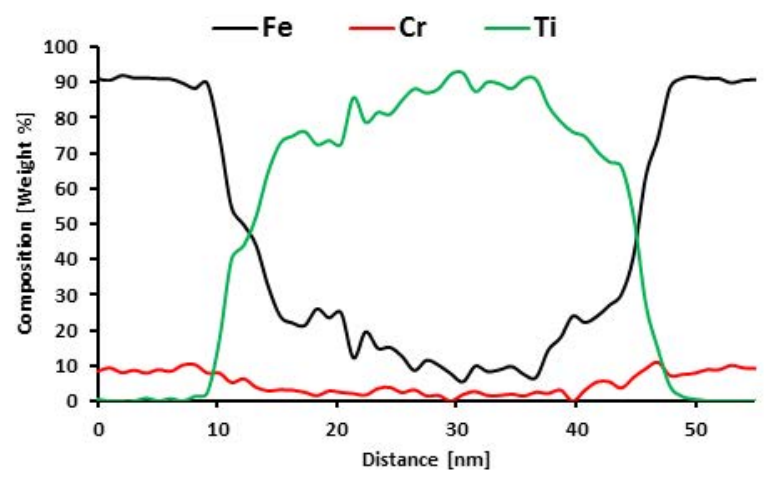

Figure 4-16: ADF images and EDS line scans of two different nanoprecipitates found in the titanium-coated sample irradiated at $700^{\circ} \mathrm{C}$. (a and b) the precipitate close to the coating is enriched in titanium and depleted in iron, (c and d) the precipitate found along the grain boundary seems to be a metallic inclusion of titanium.

As can be seen in Figure 4-16, the near-coating nanoprecipitates are compositionally similar to precipitates found in the titanium-coated samples irradiated at $500^{\circ} \mathrm{C}$ (Figure 4-10). Grain boundary nanoprecipitates could have resulted from a metallic inclusion of titanium, and their composition also resembles the nanoprecipitates found in the grain boundaries of the titanium-coated sample irradiated at $500^{\circ} \mathrm{C}$ (Figure 4-9). 


\subsubsection{Yttrium-coated sample}

Figure 4-17 shows ADF images and the corresponding EDS elemental analysis line scans of the sample prepared from the yttrium-coated specimen exposed and irradiated at $700^{\circ} \mathrm{C}$, respectively.
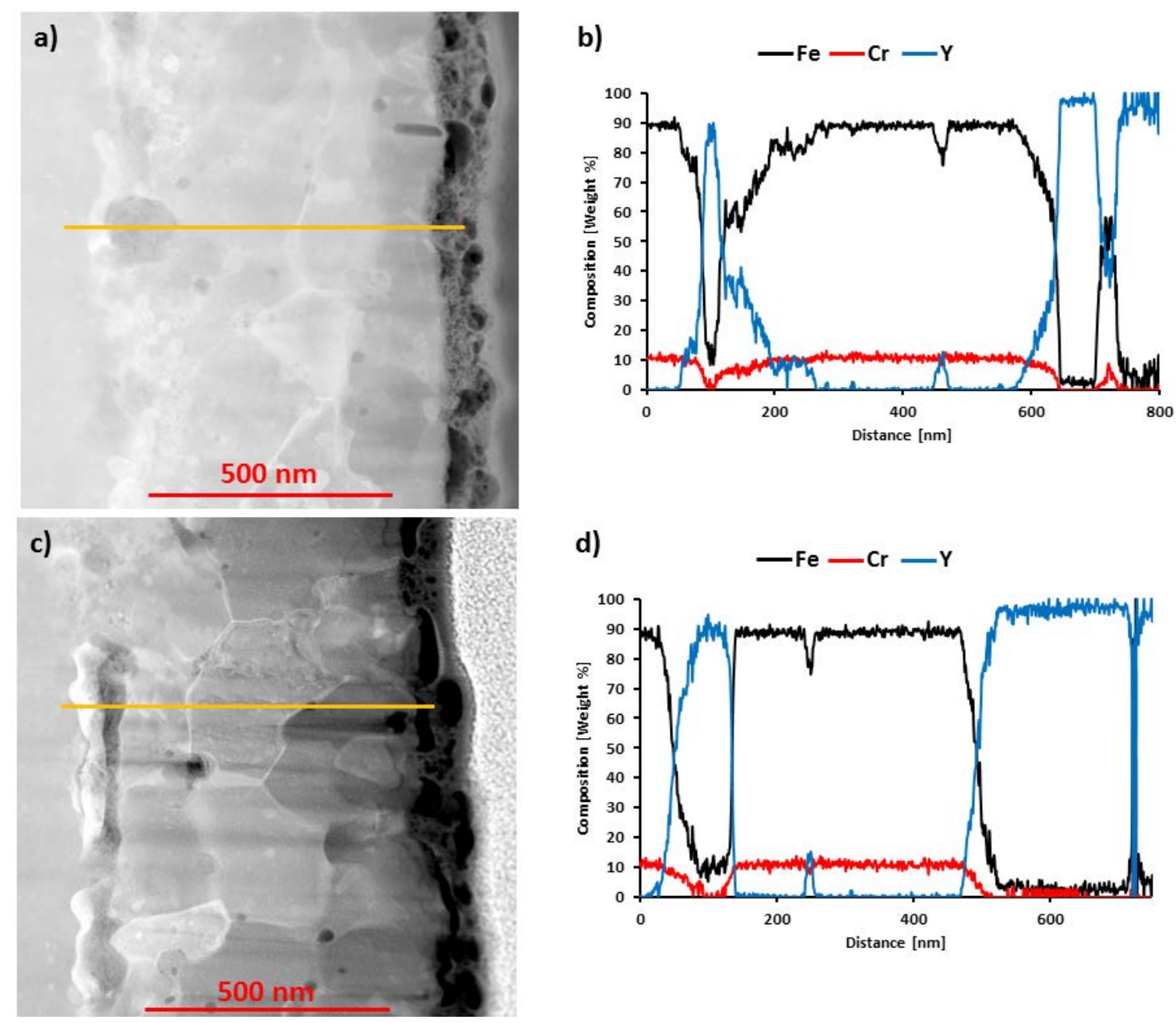

d)

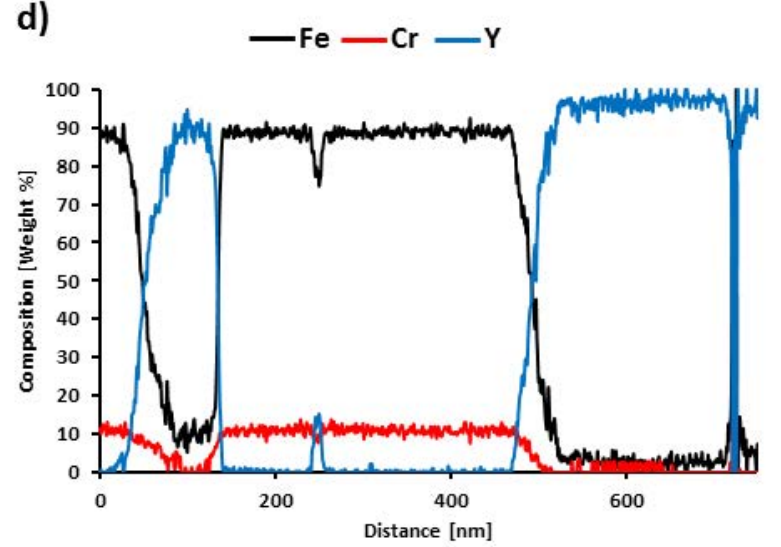

Figure 4-17: ADF images and EDS line scans of the yttrium-coated sample, (a and b) exposed at $700^{\circ} \mathrm{C}$, and (c and d) irradiated at $700^{\circ} \mathrm{C}$. Irradiation effects appear to be masked by temperature-related effects.

As with the titanium-coated sample, it is difficult to find relevant features that distinguish the exposed sample from the irradiated sample (Figure 4-17). On average, by performing different EDS line scans across the interface and up to the position of the yttrium coating, it was observed that the length of intermixed region is comparable in both the samples. By observing the EDS line profiles, it is seen that the intermixed region has a composition which is very similar to the substrate. In both the samples some nanoprecipitates were also detected and analyzed, and Figure 4-18 shows ADF images and EDS analysis performed on some of these nanoprecipitates. 

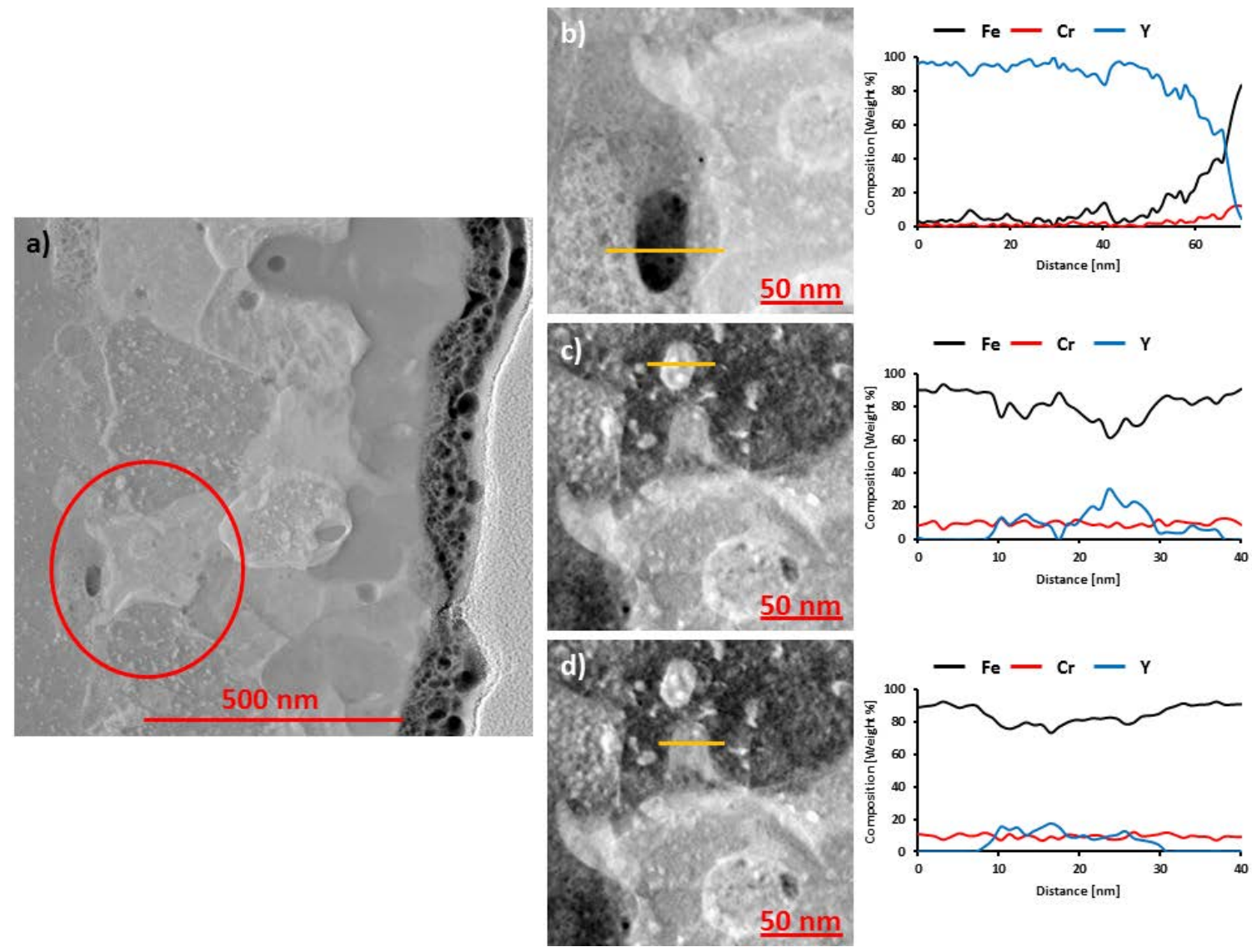

Figure 4-18: ADF images and EDS line scans of three different nanoprecipitates found in the intermixing region of the yttrium-coated and $700^{\circ} \mathrm{C}$ irradiated sample. The three precipitates are found in the region enclosed in the red circle in a). Precipitates with a brighter contrast are of differing composition from the surrounding matrix. Similar results were obtained for the yttrium-coated, $700^{\circ} \mathrm{C}$ exposed sample without irradiation.

As can be seen in Figure 4-18, high-brightness nanoprecipitates in the ADF images are enriched in yttrium and depleted in iron. Low-brightness nanoparticles appear to have the same composition as the surrounding matrix.

\subsection{As-deposited oxide thin film examination}

Figure 4-19 shows ADF images and the corresponding EDS line scans of the titanium and yttrium oxide as-deposited samples. As can be seen from Figure 4-19, the interface between the $\mathrm{Fe}-12 \% \mathrm{Cr}$ substrate and the oxide coatings is not perfectly sharp; some intermixing is occurring during the first stages of the deposition process. 

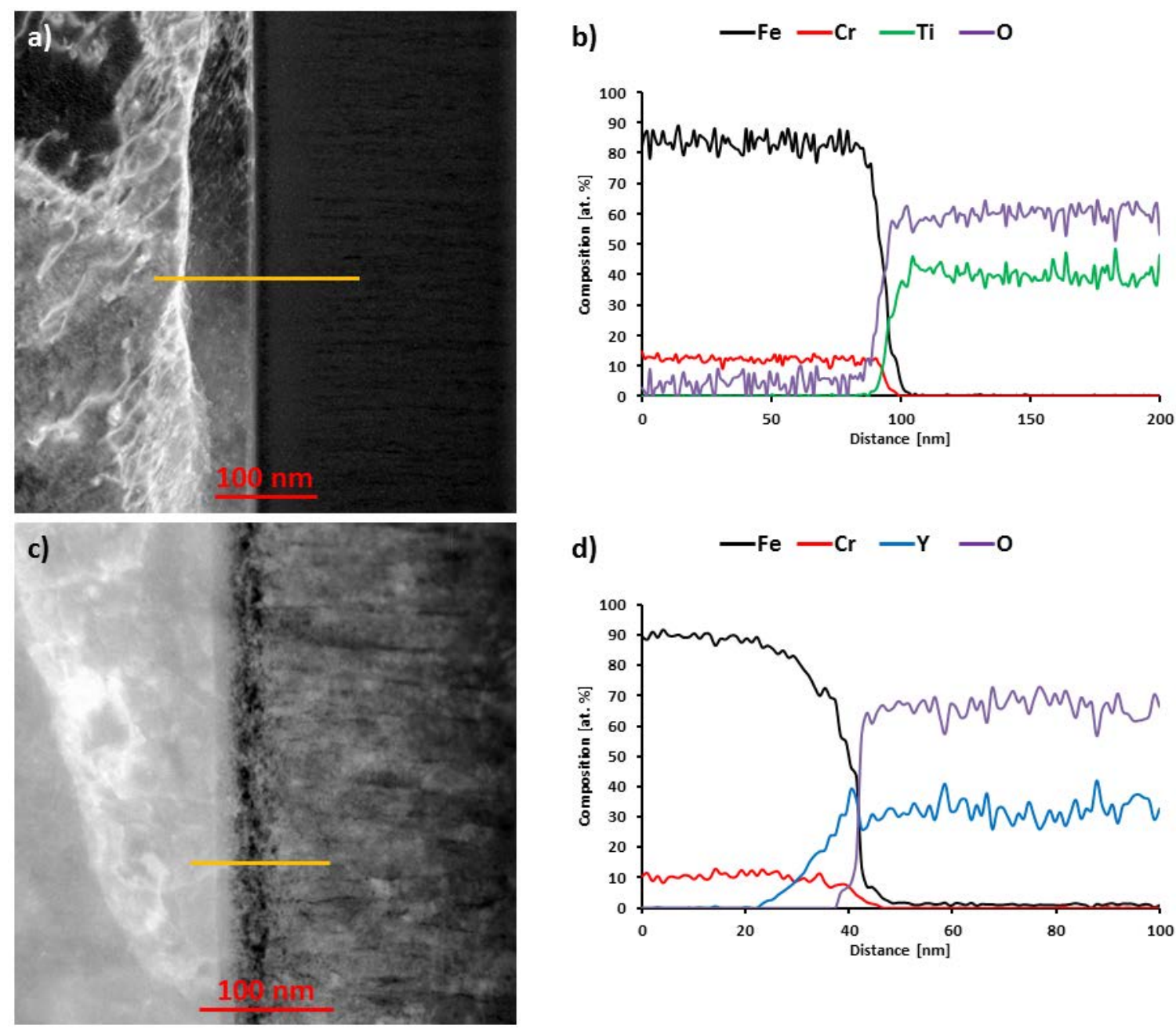

Figure 4-19: As-deposited ceramic thin film-coated samples (a and b) titanium oxide, (c and d) yttrium oxide. Yttrium is detected further into the substrate than titanium, while in each coated sample the oxygen has penetrated a comparable amount.

\subsection{Irradiated \& exposed oxide thin film examinations}

\subsubsection{Titanium oxide and yttrium oxide-coated samples irradiated at $300^{\circ} \mathrm{C}$}

\subsubsection{Titanium oxide-coated sample}

Figure 4-20 shows ADF images and the corresponding EDS elemental analysis line scans of the sample prepared from the titanium oxide-coated specimen exposed and irradiated at $300^{\circ} \mathrm{C}$, respectively. Due to the short exposure to relatively low temperatures, the chemical compositions of the irradiated and the exposed thin film-substrate interfaces are very similar to 
the as-deposited titanium oxide sample. That said, irradiation noticeably altered the structure of the interface, generating a wider intermixing region with a composition plateau of $10 \mathrm{~nm}$ width between coating and substrate.
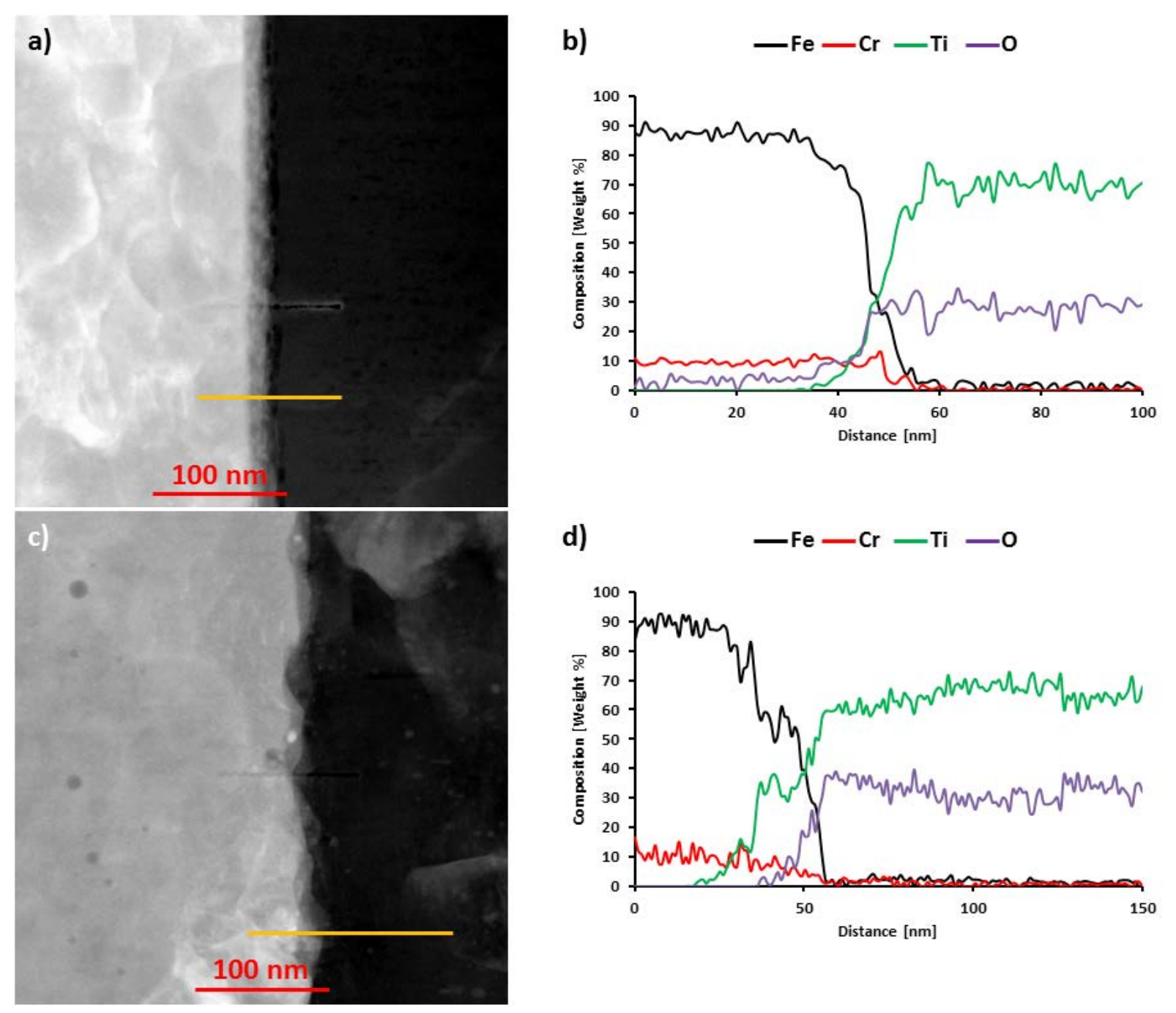

Figure 4-20: ADF images and EDS line scans of the titanium oxide-coated sample, (a and b) exposed at $300^{\circ} \mathrm{C}$, and (c and d) irradiated at $300^{\circ} \mathrm{C}$. Behavior is similar to the metalcoated samples: $300^{\circ} \mathrm{C}$ exposure for 3 hours does not significantly alter the sample from the as-deposited condition (Figure 4-19) while irradiation broadens the interface.

\subsubsection{Yttrium oxide-coated sample}

Figure 4-21 shows ADF images and the corresponding EDS elemental analysis line scans of the TEM sample prepared from the $300^{\circ} \mathrm{C}$ exposed \& irradiated yttrium-coated specimen. There is a major difference in the behavior of the yttrium oxide-coated sample compared to the titanium oxide-coated sample, namely a layer of chromium enrichment is observed in both the exposed and the irradiated regions. This layer forms within the interfacial region, directly 
adjacent the $\mathrm{Fe}-12 \% \mathrm{Cr}$ substrate. While EDS analysis can only offer semi-quantitative information about the substrate, results from a scan suggest that the exposed region's Crenriched layer manifests as a distinct layer of $\mathrm{Cr}_{2} \mathrm{O}_{3}$. Similar analysis on the irradiated region indicates the magnitude of Cr-enrichment has been suppressed such that it does not form a visibly distinct layer. As with the $300^{\circ} \mathrm{C}$ metallic film samples, and with the comparably treated titanium oxide sample, irradiation has caused the interface width to increase and support a composition plateau. Finally, the irradiation changed the stoichiometry of the coating, as compared to the as-deposited and the $300^{\circ} \mathrm{C}$ exposed conditions.
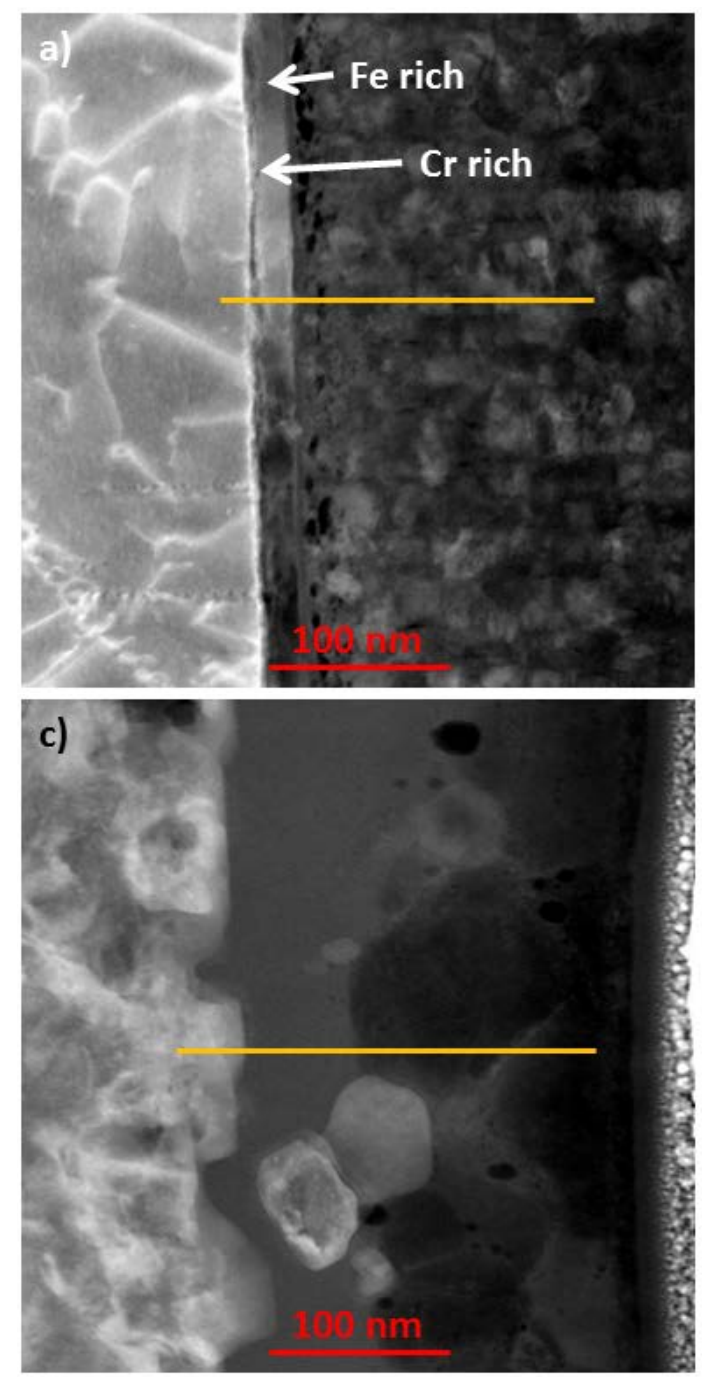

b)

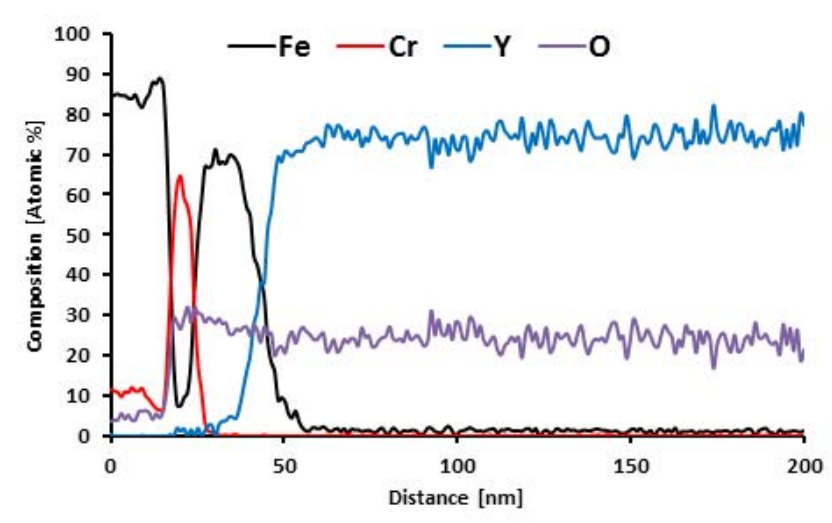

d)

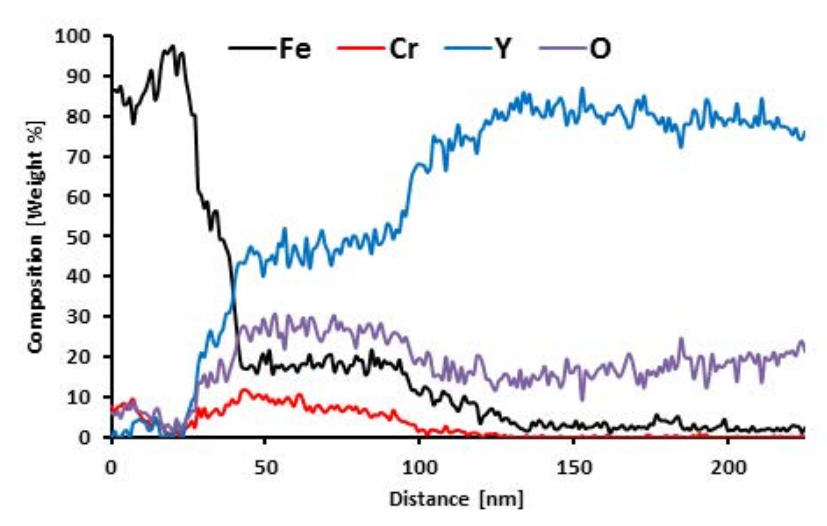

Figure 4-21: ADF images and EDS line scans of the yttrium oxide-coated sample, (a and b) exposed at $300^{\circ} \mathrm{C}$ and (c and d) irradiated at $300^{\circ} \mathrm{C}$. As opposed to the other thin film coated samples, the exposure for a few hours at $300^{\circ} \mathrm{C}$ does influence the sample morphology: chromium enrichment at the boundary is evident. 

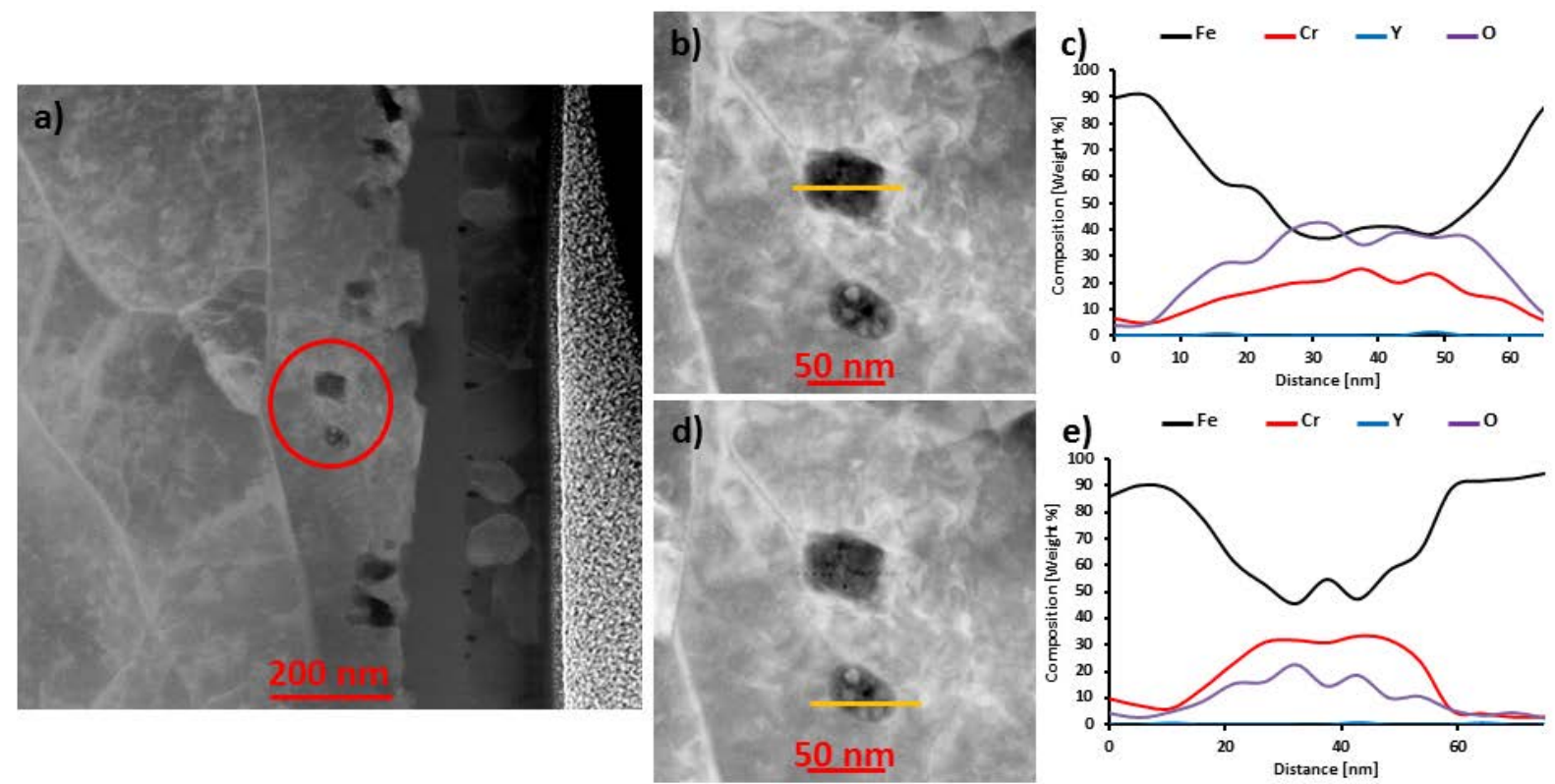

Figure 4-22: ADF images and EDS line scans of two nanoprecipitates buried in the Fe$12 \%$ Cr substrate after irradiation at $300^{\circ} \mathrm{C}$ of the yttrium oxide-coated sample. The two precipitates are circled in red in a) and the corresponding EDS line scans are shown in c) and d). The two nanoparticles are enriched in both chromium and oxygen.

A close look at the irradiated sample reveals the presence of small precipitates, shown in Figure 4-22 .The composition of the subsurface precipitates indicates they are enriched in oxygen and chromium with a reduced iron quantity, which suggests chromium oxide particles or perhaps an iron-chromium spinel. Small particles which show a brighter contrast as compared to the surrounding matrix were also found in the intermixing region. Figure 4-23 shows two of these particles and the corresponding EDS line scans. 

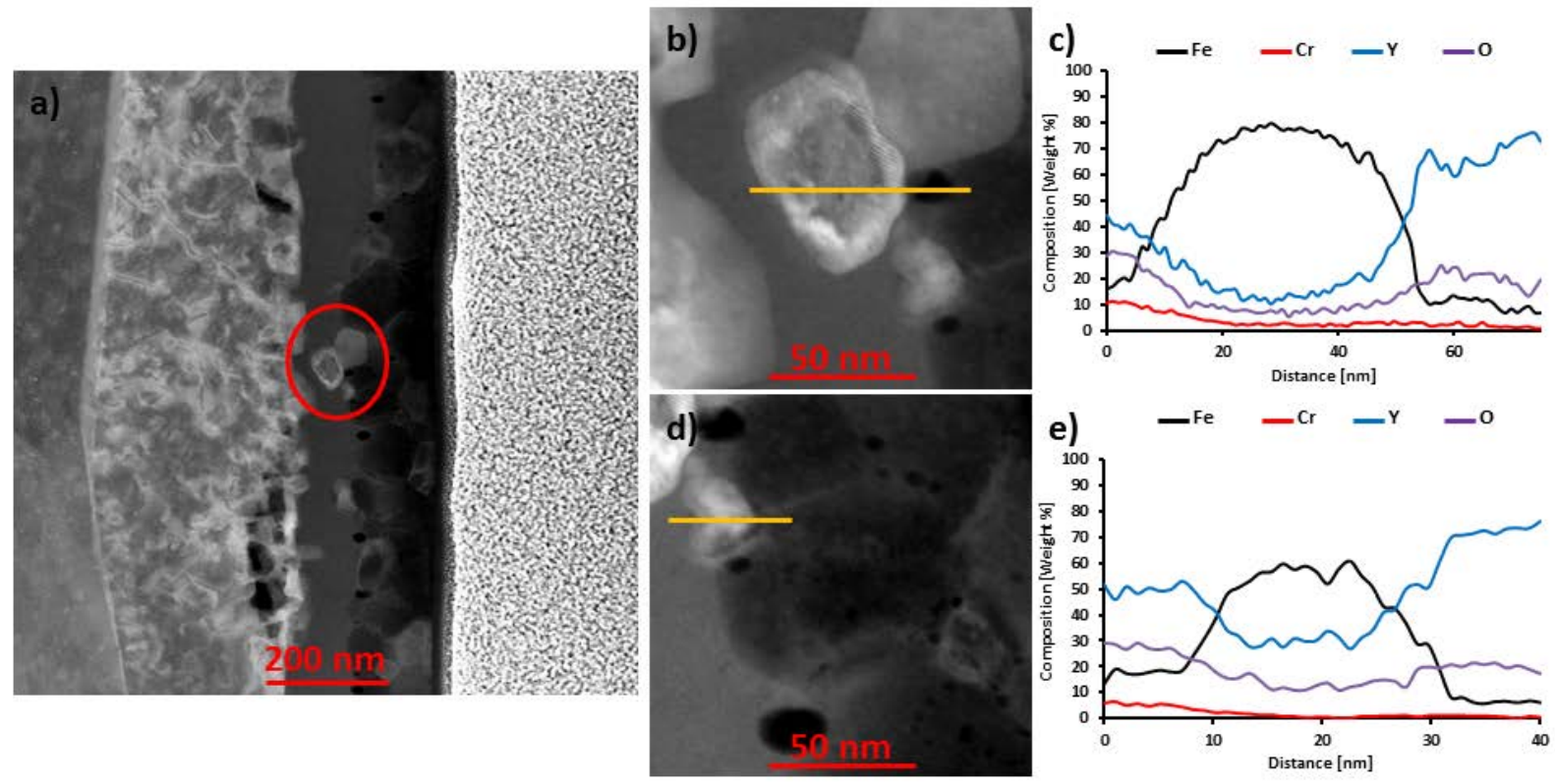

Figure 4-23: ADF images and EDS line scans of two different nanoprecipitates found in the intermixed region of the yttrium oxide-coated $\mathrm{Fe}-12 \% \mathrm{Cr}$ substrate after irradiation at $300^{\circ} \mathrm{C}$. The two precipitates circled in red in a) are magnified in b) and d), and the respective EDS line scans are shown in c) and e). The two particles exhibit similar iron enrichments.

\subsubsection{Titanium oxide and yttrium oxide-coated samples irradiated at $500^{\circ} \mathrm{C}$}

\subsubsection{Titanium oxide-coated sample}

Figure 4-24 shows ADF images and the corresponding EDS elemental analysis line scans of the sample prepared from the titanium oxide-coated specimen exposed and irradiated at $500^{\circ} \mathrm{C}$, respectively. 

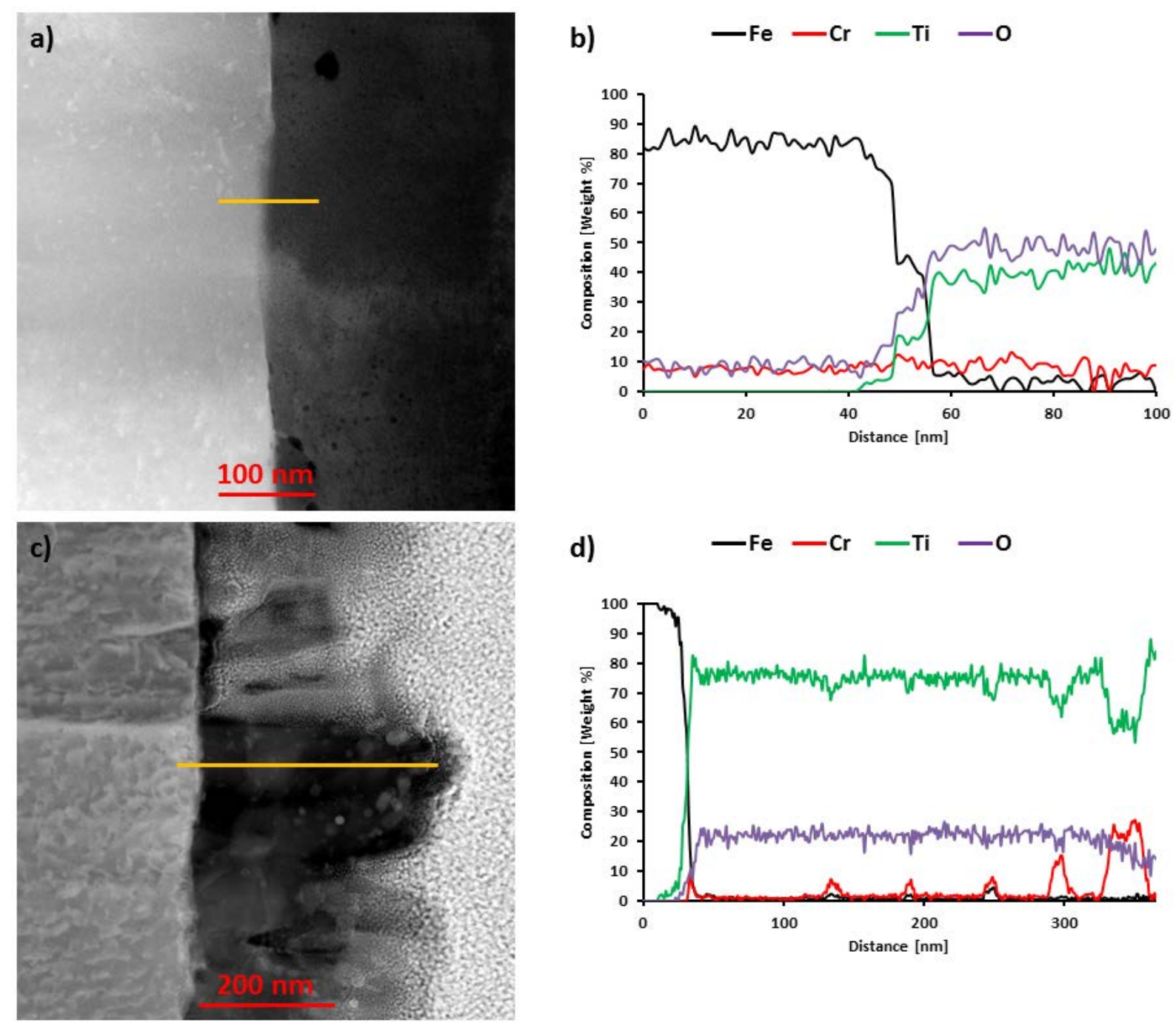

Figure 4-24: ADF images and EDS line scans of the titanium oxide-coated sample (a and b) exposed at $500^{\circ} \mathrm{C}$ and (c and d) irradiated at $500^{\circ} \mathrm{C}$. Irradiation with nickel ions sputtered away part of the coating, and determined the formation of small Cr rich particles.

As can be seen from Figure 4-24, the exposure for a few hours at $500^{\circ} \mathrm{C}$ oxidizes the coating in comparison to the as-deposited case. Combining nickel ion irradiation with the thermal exposure results in sputtering of the thin film, thinning the coating and altering the $\mathrm{Cr}$ diffusion profile. The new $\mathrm{Cr}$ diffusion profile is no longer homogenous within the coating, but rather tends to form precipitates in the intermixed region. EDS analysis of a nanoprecipitate (Figure 4-25) indicates the nanoprecipitate is enriched in chromium at the expense of titanium. Overall, the oxide coating from the irradiated region is depleted in oxygen relative to the thermally exposed region. 

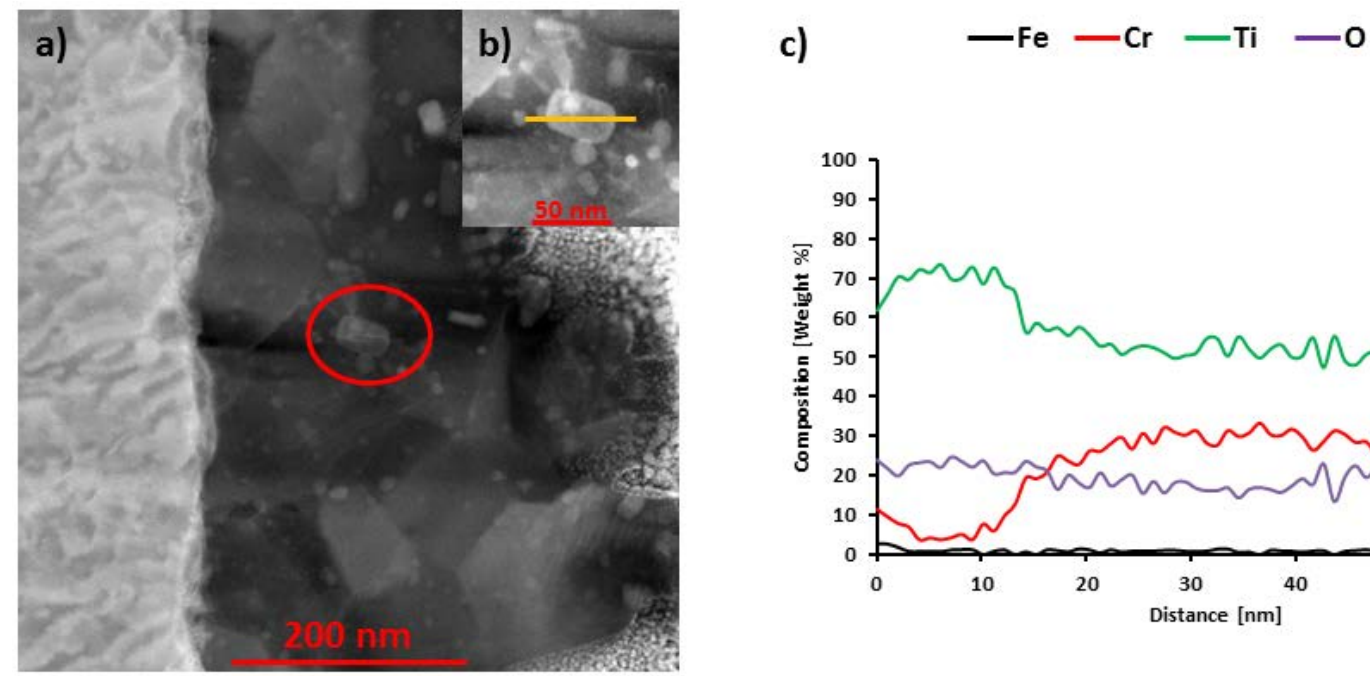

Figure 4-25: ADF images and EDS line of a nanoprecipitate found the titanium oxidecoated sample after irradiation at $500^{\circ} \mathrm{C}$. The precipitate circled in red in a) is shown magnified in inset b), along with an EDS line scan in c). The precipitate is enriched in chromium and depleted in titanium.

\subsubsection{Yttrium oxide sample}

Figure 4-26 shows ADF images and the corresponding EDS line scans of the sample prepared from the yttrium oxide-coated specimen following the $500^{\circ} \mathrm{C}$ experiment. As can be seen from Figure $4-26$, similar to what is observed after the $300^{\circ} \mathrm{C}$ exposure, a chromiumenriched region is detected close to the interface followed by an iron-enriched region. Exposure to the radiation field again results in compositional differences between the samples' thin chromium layers. As in the $300^{\circ} \mathrm{C}$ samples, the irradiated layer is considerably wider than the layer in the exposed sample. 

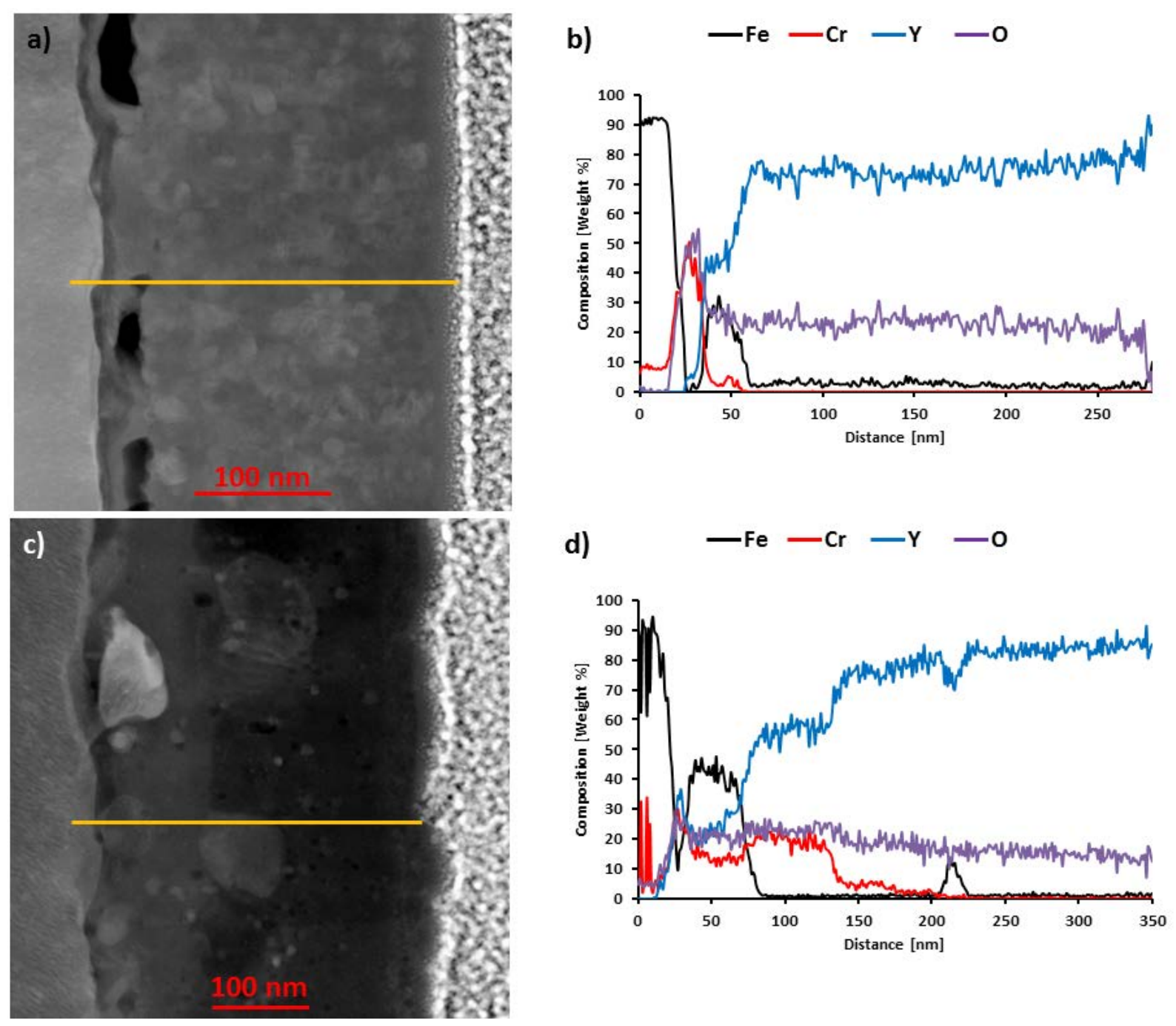

Figure 4-26: ADF images and EDS line scans of the yttrium oxide-coated sample exposed at $500^{\circ} \mathrm{C}$ (a and b), and irradiated at $500^{\circ} \mathrm{C}$ ( $\mathrm{C}$ and $d$ ). The exposure at $500^{\circ} \mathrm{C}$ forms a nearinterface chromium-enriched region, followed by an iron-enriched region. The radiation field suppresses the formation of these layers, promoting the formation of a thicker layer with an altered chemical composition.

\subsubsection{Titanium oxide and yttrium oxide-coated samples irradiated at $700^{\circ} \mathrm{C}$}

\subsubsection{Titanium oxide-coated sample}

Figure 4-27 shows ADF images and the corresponding EDS elemental analysis line scans of the sample prepared from the titanium oxide-coated specimen exposed and irradiated at $700^{\circ} \mathrm{C}$, respectively. 

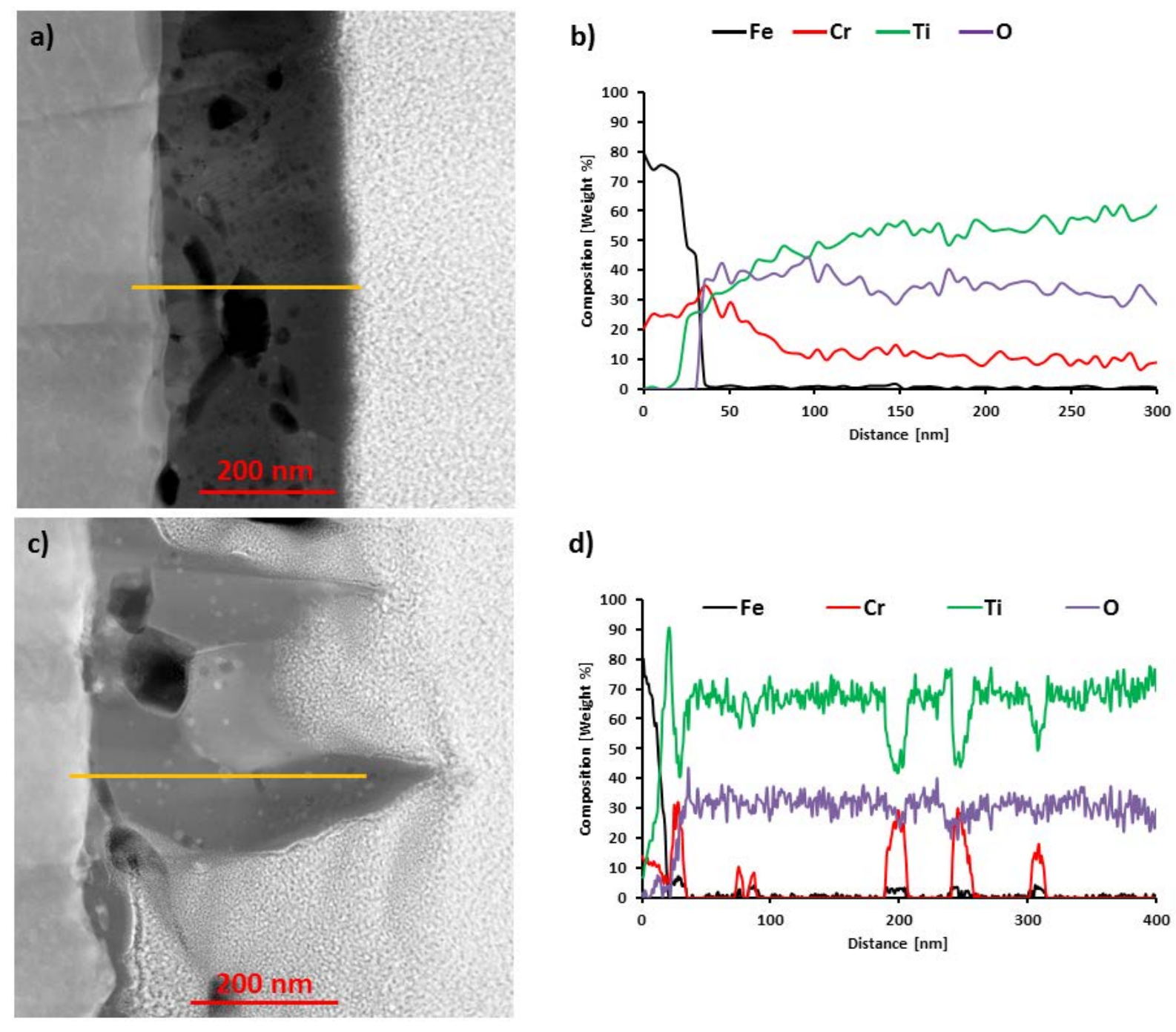

d)

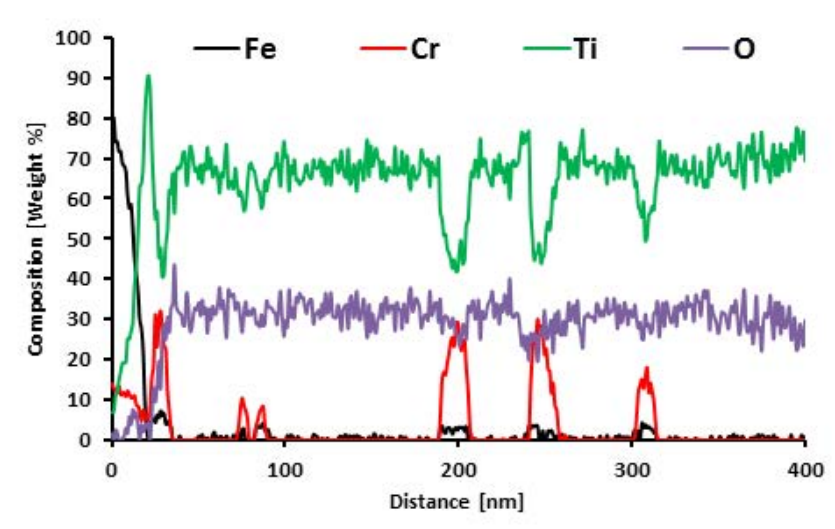

Figure 4-27: ADF images and EDS line scans of the titanium oxide-coated sample exposed at $700^{\circ} \mathrm{C}$ (a and b), and irradiated at $700^{\circ} \mathrm{C}$ ( $\mathrm{c}$ and $\mathrm{d}$ ). The exposure at $700^{\circ} \mathrm{C}$ does not cause broaden the substrate-coating interface despite the observed diffusion of

Cr into the coating. Irradiation again sputters part of the coating, preventing the homogenous ingress of chromium.

As can be seen from Figure 4-27, after the $700^{\circ} \mathrm{C}$ exposure the interface is still sharp despite the diffusion of chromium. No precipitates are found in the exposed coating. Irradiation at the same temperature sputters away significant portions of the thin film, modifying the diffusion profiles of both iron and chromium and promoting the formation of titanium-deficient nanoprecipitates. 


\subsubsection{Yttrium oxide-coated sample}

Finally, Figure 4-28 shows ADF images and the corresponding EDS elemental analysis line scans of the sample prepared from the yttrium oxide-coated specimen exposed and irradiated at $700^{\circ} \mathrm{C}$, respectively.
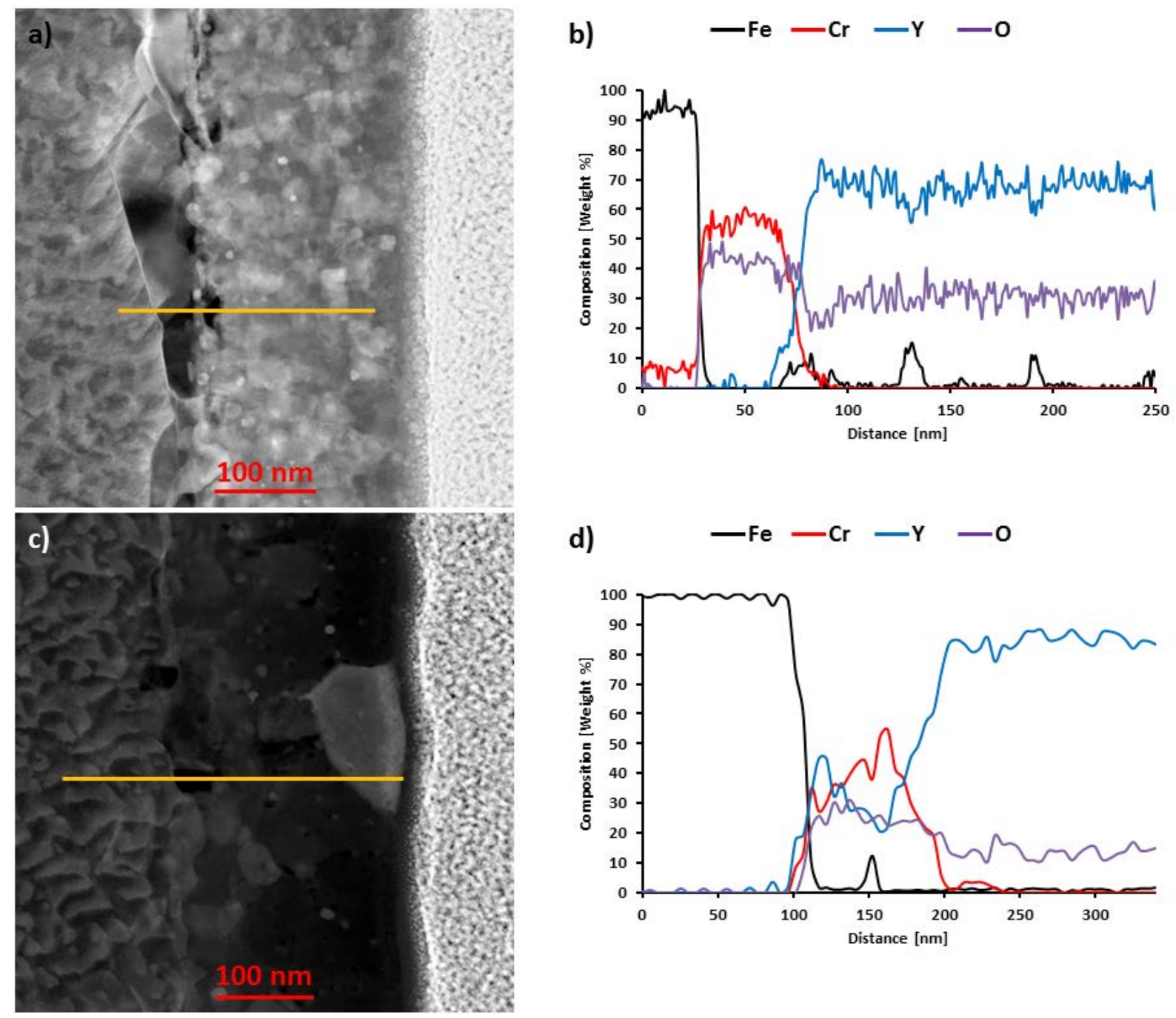

Figure 4-28: ADF images and EDS line scans of the yttrium oxide-coated sample exposed at $700^{\circ} \mathrm{C}$ (a and b), and irradiated at $700^{\circ} \mathrm{C}$ ( $\mathrm{C}$ and d). Exposure at $700^{\circ} \mathrm{C}$ forms large, $\mathrm{Cr}$ rich nanoprecipitates close to the interface. Irradiation at the same temperature results in smaller near-interface nanoprecipitates that are now $Y$-rich.

As can be seen from Figure 4-28, exposure at $700^{\circ} \mathrm{C}$ causes the formation of big nanoprecipitates enriched in chromium and lying close to the interface. The diffusion profile of chromium suggests the element is migrating to the coating (the near-interface chromium content in the substrate is smaller than the nominal composition of $12 \mathrm{wt} \%$ ) to form a chromiumenriched region on the thin film side of the interface. Irradiation at the same temperature seems to favor this behavior: the content of chromium on the substrate side is zero (Figure 4-28c), and 
on the coating side the chromium-enriched region is wider. Unlike in the exposure-only sample, yttrium is detected within the substrate. Very few precipitates were found post-irradiation, and a closer look at two of those detected can be found in Figure 4-29. Nanoprecipitates appearing brighter in the ADF images are enriched in iron and depleted in yttrium, while the dark nanoprecipitates are indistinguishable from the matrix.
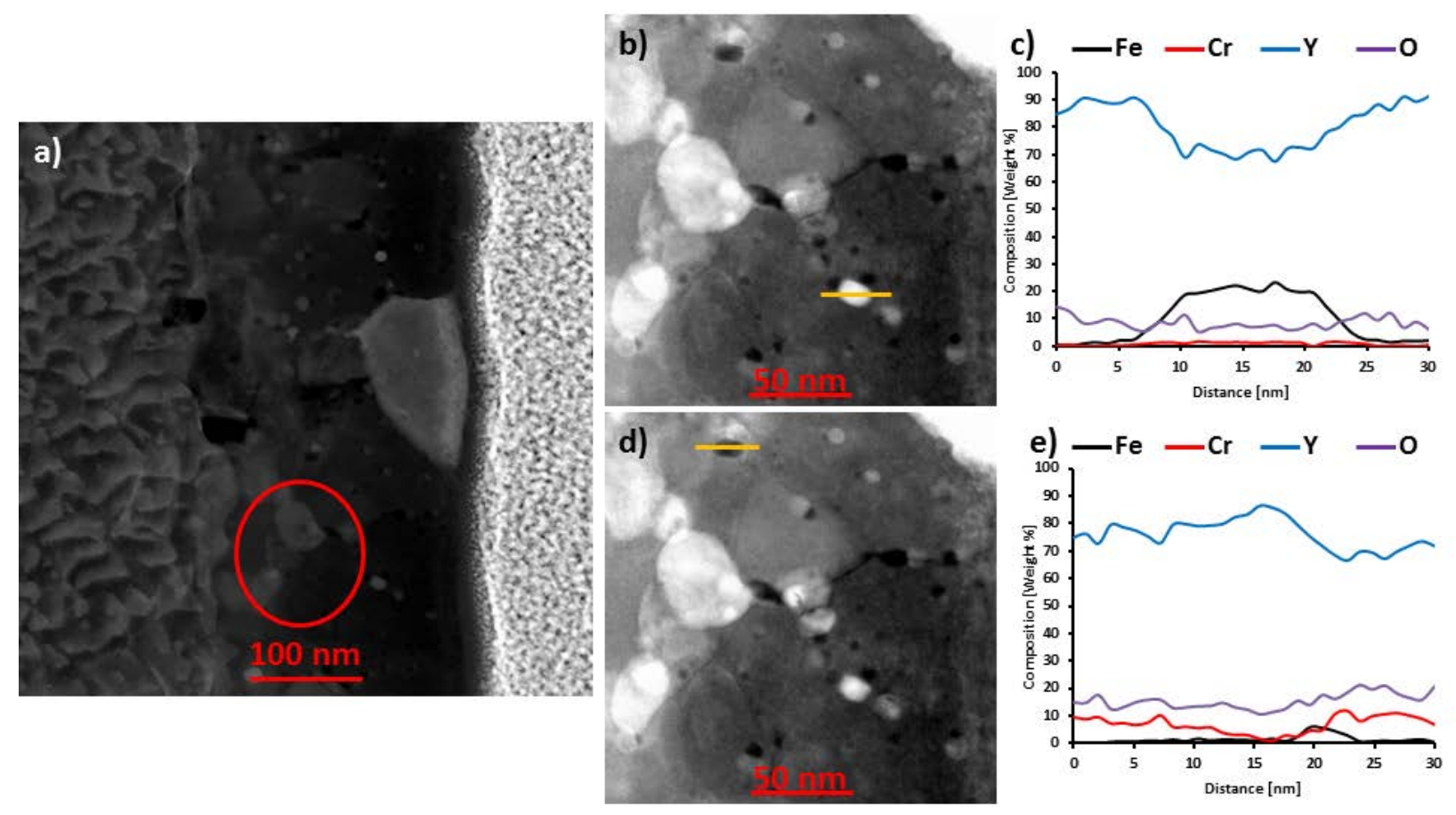

Figure 4-29: ADF images and EDS line of a nanoprecipitate found the yttrium oxidecoated sample after irradiation at $700^{\circ} \mathrm{C}$. The precipitate circled in red in a) is shown magnified in b) along with an EDS line scan in c). The precipitate is enriched in chromium and depleted in titanium.

\subsection{Simulation work}

Molecular dynamics (MD) simulations were performed to develop a radiation damage modeling strategy for Fe-Cr alloys. The molecular dynamics simulation technique was chosen over the more advanced technique based on density functional theory (DFT) due to the scope of the calculation involved - DFT is extremely time-expensive, while existing simulation programs for MD are capable of handling orders of magnitudes more atoms than DFT while maintaining reasonable simulation speed on accessible hardware. This research was performed using the compute resources and assistance of the UW-Madison Center for High Throughput Computing (CHTC) in the Department of Computer Sciences. The CHTC is supported by UW-Madison and the Wisconsin Alumni Research Foundation, and is an active member of the Open Science Grid, which is supported by the National Science Foundation and the U.S. Department of Energy's Office of Science. Additional compute resources provided by the Ohio State University were utilized. Visual interpretation of the textual simulation output was made possible with the VMD Visual Molecular Dynamics software (90). 


\subsubsection{Irradiation damage studies on $\mathrm{Fe}-12 \% \mathrm{Cr}$ alloy}

To initiate irradiation damage studies via computational simulation, one must take some liberties with the simulation program. A typical irradiation program involves incident particles (neutrons, ions, etc.) with very high energy penetrating the substrate and interacting with the substrate in some manner (as previously described in Section 1.2.2). Rather than attempt to simulate the interaction of the irradiating species with the substrate and the subsequent interactions of these primary knock-on atoms (PKAs) with the other atoms of the substrate, the simulation is restricted to consider only the PKA-substrate interactions. This restriction enables selection of a larger timestep and simplifies the constraints on the interatomic potentials needed to describe the system by eliminating the highest-energy species, which is often not otherwise found in the substrate (except in the case of self-irradiation experiments). This choice helps make MD computationally viable for studying radiation damage.

For a given grain microstructure, there are two parameters of importance in irradiation damage studies: PKA energy, and PKA direction. An increase in PKA energy is expected to increase the overall resultant damage, while changes in the orientation angle with the grain will alter the time and length scale of the damage cascade. When the PKAs align with low-atomdensity crystallographic pathways, a "channeling" phenomenon can occur that should increase both the time and length scale of the damage cascade, as the ions travel further between collisions with matrix atoms. Conversely, when the irradiation direction is along high density directions, the damage cascade is expected to initiate more rapidly and more strongly.

In this work, the effect of one and two PKAs on the extent of defects were studied and compared. To perform the simulations, the Large-scale Atomic/Molecular Massively Parallel Simulator (LAMMPS) package was used (91). Interatomic potential data for the Fe-Cr sample was supported by the LAMMPS potentials library; a concentration-dependent embedded-atommethod potential (CD-EAM) was used (92). The concentration dependence of this potential helps reproduce the Fe-Cr interactions in the low-chromium region. A total of 31,250 atoms were simulated, with 27,158 atoms being $\mathrm{Fe}$ and the remaining 4,092 atoms being $\mathrm{Cr}$, and with periodic boundary conditions established on all 6 faces. Thermal management was performed by implementing a $300 \mathrm{~K}$ Langevin thermostat on a shell along the periodic borders of the simulation box. Within the shell, no velocity regulation was performed, allowing the core atoms to interact according to the governing CD-EAM potential. An energetic primary knock on atom (PKA) was chosen at the center of the simulation cell and was changed to two PKAs separated by a distance of $5 \AA$; the cascade process was then integrated with a varying time step in the range of $0.001 \mathrm{fs}$ to $0.1 \mathrm{fs}$ up to a total time of $4 \mathrm{ps}$. Energies between 0.5 and $4 \mathrm{keV}$, based on literature and experiments (93), were used for the initial energy of the PKA atoms. After 400 time steps, the irradiated system was quenched to room temperature $(300 \mathrm{~K})$ and the resulting structure was analyzed for defects. The next step was to characterize the defects that have been produced as a result of the irradiation damage. A sharp difference was observed when the defects were analyzed in the system after irradiation with one or two PKAs, both in terms of the number of defects and their nature. Figure 4-30 shows an orthographic representation of the defects that have been produced when the irradiation is stimulated by means of two PKAs. 
b)

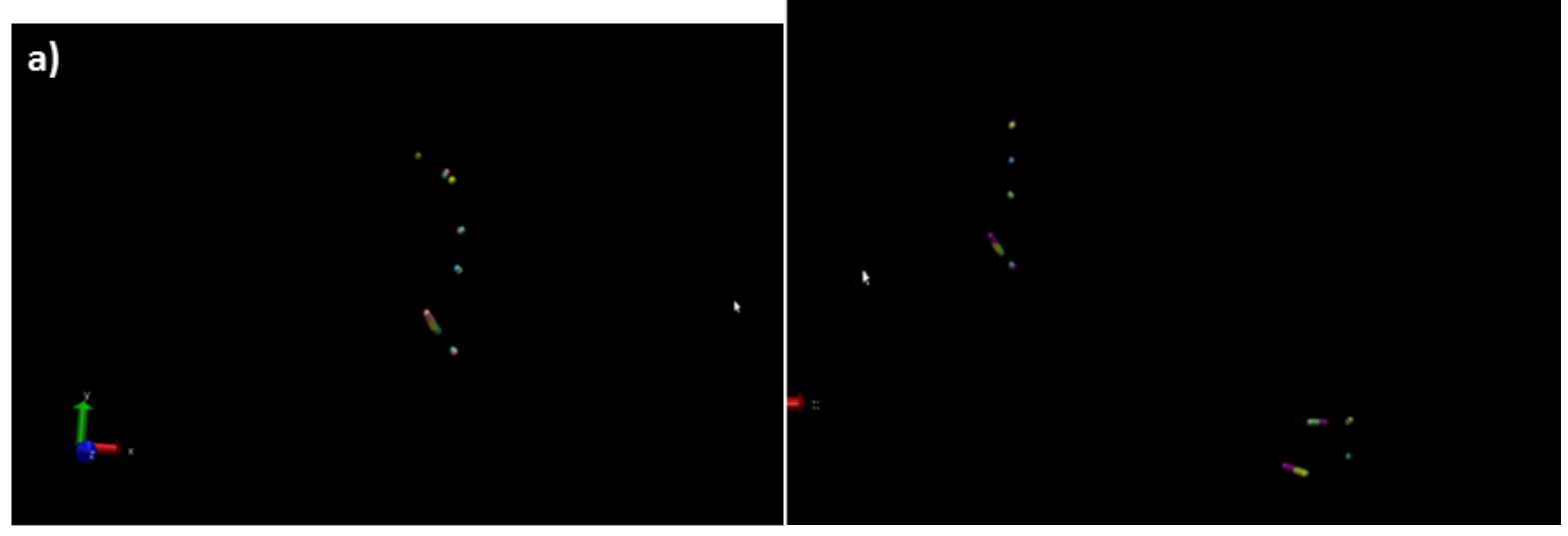

Figure 4-30. Final defect configuration after two PKAs were injected into the Fe-Cr binary alloy. Matrix atoms are not visualized.

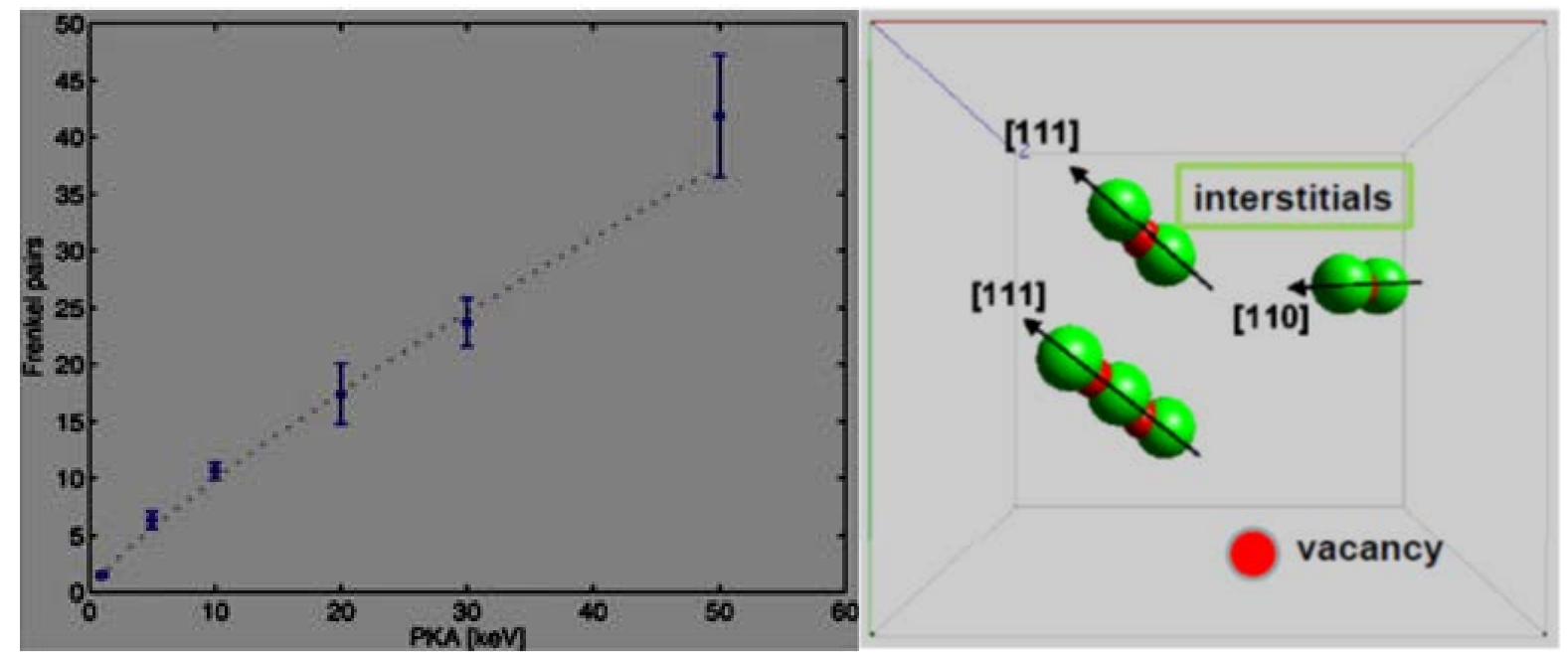

Figure 4-31. (I) Graphical representation of the relationship between the number of Frenkel pairs and the PKA energy, as expressed by Equation 4-1. (r) The vacancy and interstitial configurations likely in an irradiated Fe-12\% Cr binary alloy.

Since there was a curiosity to study the number of Frenkel Pairs with the increase of the energy of PKA atoms, a set of simulations was also run assigning a different energy to the PKAs. The number of pairs obtained for different energies of PKA was determined and fitted to an exponential law given by:

$$
N=a E_{P K A}^{b}
$$

Equation 4-1

where $N$ is the number of FPs, $E_{P K A}^{b}$ is the primary knock on energy, $a$ and $b$ are the fitting parameter which are found to be equal to $1.49 \pm 0.15$ and $0.82 \pm 0.03$ respectively. Figure 4-31 fits Equation 4-1 to the experimental data, and the vacancies-interstitials configuration observed. 


\subsubsection{Oxidation of bcc Iron via MD Simulation}

Simulation of a pure iron substrate has been conducted utilizing the ReaxFF reactive inter-atomic potential for iron, carbon, oxygen, and water derived by Aryanpour (94) and implemented in LAMMPS via the USER-REAX/C module (95). These simulations are conducted in order to generate reasonable interfaces between the iron and oxygen atoms without imposing a user-chosen phase, i.e. to create a naturally evolved interface. The oxidation rate of the substrate is expected to be several orders of magnitude greater than real-world cases due to the limitation of computational resources - it is simply not feasible using current computational technologies to oxidize a pure substrate and form a reasonable thickness oxide layer, given that such a process requires a physical time on the order of seconds to hours.

The simulation structure is detailed as follows. First, 100,000 iron atoms were arrayed in a 50x50x20 unit cell slab geometry, with a vacuum region above and below the sample, forming a simulation box measuring $142.75 \AA$ x $142.75 \AA$ x $571 \AA$. The sample was equilibrated (in 3 separate simulations) to the working temperatures of $300^{\circ} \mathrm{C}, 500^{\circ} \mathrm{C}$, and $700^{\circ} \mathrm{C}$. Following the established equilibration procedure from prior experiments, these samples were then checked for thermal and geometric stability by allowing free-form (no thermal or pressure control) simulation for 2 picoseconds (2,000 time steps). Lastly, a thin (11.42 $\AA$ ) region in the middle of the sample is assigned to a thermostat bath. Any atoms within this region will act as a heat sink for energy released during oxidation, as would be experienced in a natural setting in the form of the bulk sample.

The samples were then oxidized progressively to minimize any unphysical results that would stem from the simultaneous introduction of hundreds or thousands of oxygen atoms: every 200 time steps 4 atoms of oxygen are introduced to the system, 2 of which are placed randomly within a region above the sample, and two of which are placed randomly within a region below the sample. After introducing the atoms, all oxygen atoms within the introduction regions are reinitialized to the desired simulation temperature with randomly oriented velocities. No other thermal control beyond maintenance of the thin heat sink is performed.

To determine if the choice of timestep would play a role in the developed geometry, a second set of geometrically identical simulations using a reduced timestep of 0.25 femtoseconds (fs) was developed. A slightly elevated oxygen introduction rate of 4 per 125 fs (4 atoms every 500 timesteps) was used compared to the 1 fs-timestep simulations. There was no discernable structural difference between the 0.25 and 1.0 fs timestep choice, so the longer timestep simulations were continued. After 278,400 timesteps, the rate of oxygen introduction was increased from 4 per 200 timesteps (4 per 200 femtoseconds, fs) to 20 per 200 fs to decrease the time until a constant-atom oxidation simulation was obtained. Figure 4-32 catalogs the development of this system after 67,000 and 323,000 time steps. It is apparent from viewing the figures that dissolved oxygen preferentially occupies the interstitial sites of the iron matrix.

Due to time limitations, the oxidation simulations were discontinued in favor of conducting the irradiation simulations described in Section 4.5.1. 

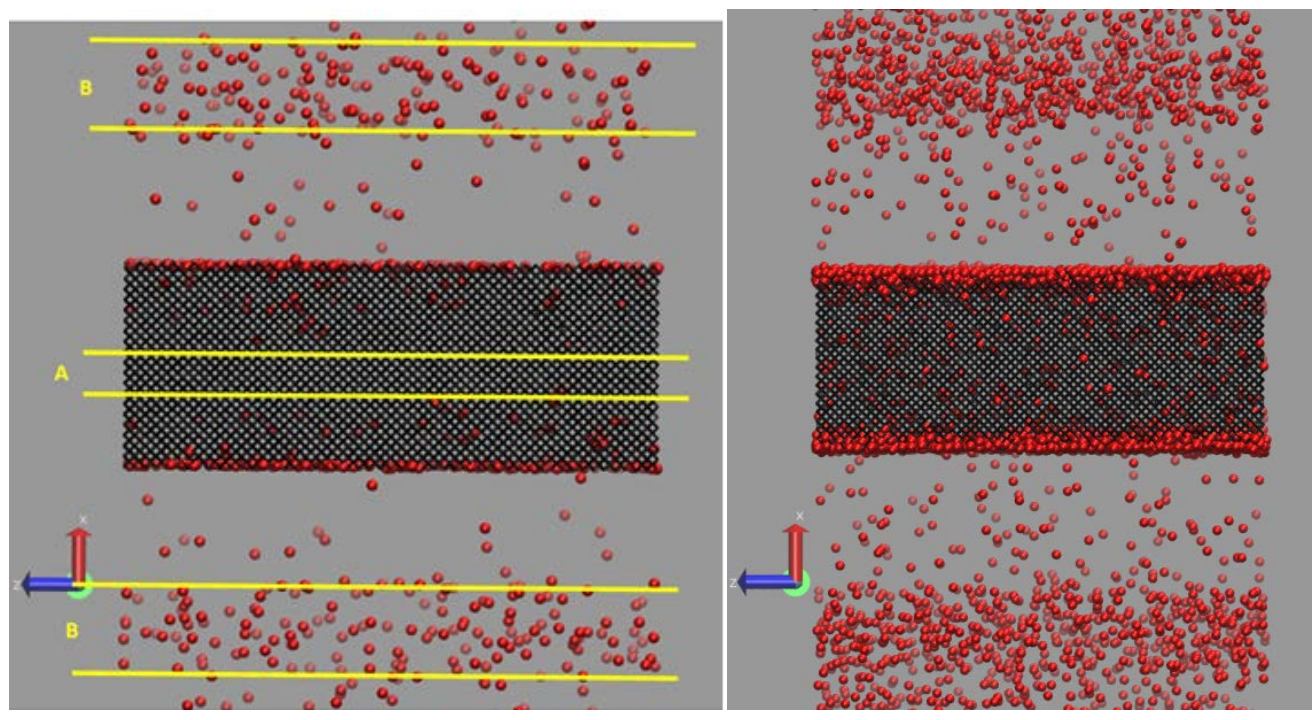

Figure 4-32: $300^{\circ} \mathrm{C}$ iron surface oxidation simulation after (I) 67 and (r) 323 picoseconds of simulation using Aryanpour's Fe-O-C-H ReaxFF potential. Region $A$ is thermally controlled, and the areas denoted by $B$ are the oxygen introduction regions. Iron atoms are at the intersections of the black bonds, and oxygen atoms are shown as red spheres of arbitrary size. The simulation box extends vertically (not shown). 


\section{CHAPTER 5 \\ Discussion}

\subsection{As-deposited samples}

The as-deposited thin film samples were prepared and analyzed as reference samples for the unirradiated case. The sharpness of the interface is calculated mathematically by calculating the effective interdiffusion coefficient, treating the system as an irradiated bilayer. The concentration profiles obtained from the STEM-EDS analysis can be fitted with special functions. The interdiffusion coefficient calculated with this treatment does not have the usual physical meaning for these samples, but it can be used as a quantitative factor to evaluate the extent of the substrate-thin film interface.

In general, the interdiffusion coefficient can be calculated as though the diffusional processes occur between two semi-infinite solids. Starting from Fick’s second law:

$$
\frac{\partial C}{\partial t}=D \frac{\partial^{2} C}{\partial x^{2}}
$$

Equation 5-1

The concentration profiles for irradiated bilayers can be fitted by both the error function and its complement, yielding the solution of Equation 5-1 for semi-infinite slab sources:

$$
C(x, t)=\frac{C_{0}}{2} \operatorname{erf}\left(\frac{\left(x-x_{0}\right)}{\sqrt{4 \widetilde{D} t}}\right)
$$

Equation 5-2

where $C(x, t)$ is the concentration profile of the diffusing atoms, erf is the error function, $C_{0}$ is the initial concentration, usually considered equal to $1, x_{0}$ is the center of the mixing profile, $\widetilde{D}$ is the interdiffusion coefficient and finally $t$ is the time. The error function is defined as:

$$
\operatorname{erf}(x)=\frac{1}{\sqrt{\pi}} \int_{0}^{x} e^{-t^{2}} d t
$$

To interpolate the experimental data acquired, the software Origin Lab 9.1 was used. The software can perform integral fitting to determine the relevant parameters of the fitting function, which are $x_{0}$ and $\sqrt{4 \widetilde{D} t}$. Two other parameters are used to fit the function, but they serve only to shift and scale the fitting functions along the y-axis and therefore they do not have a physical meaning. As mentioned above, in the case of the as-deposited samples the interdiffusion coefficient, $\widetilde{D}$, does not have its usual physical meaning and instead is being used to quantify the extent of the substrate-thin film interface (Figure 5-1). 


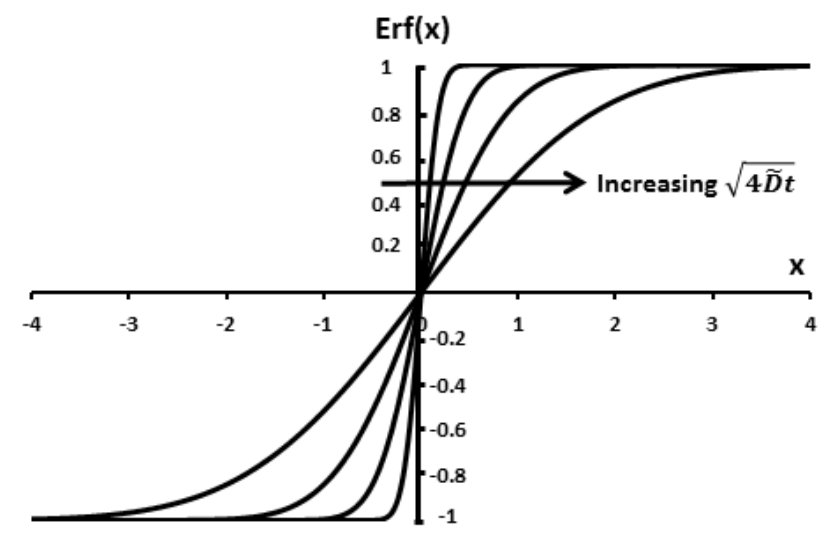

Figure 5-1: The relationship between the error function, erf $\left(\frac{(x)}{\sqrt{4 \widetilde{D} t}}\right)$, and various relative magnitudes of the parameter $\sqrt{4 \widetilde{D} t}$.

The as-deposited samples' composition profiles were fitted with these special functions and the results obtained are shown in Figure 5-2. Excellent fit of the functions (lines) to the raw data (dots) is observed.
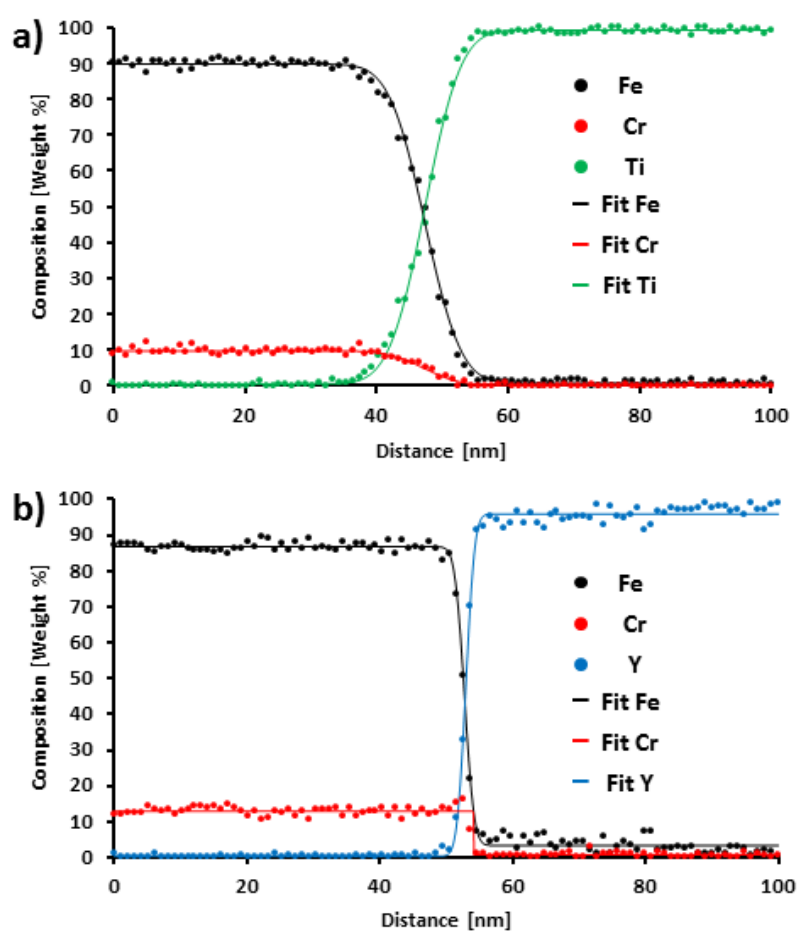

Figure 5-2: Erf and Erfc fitting functions for the iron and titanium concentration profile of the titanium and yttrium as-deposited samples. The raw composition profiles are shown as dots. 
Raw data from the as-deposited samples is available for 3 line scans, and thus the fitting calculations were performed on each individual line scan. The resultant interdiffusion coefficients were averaged, and the values are shown in Table 3, along with the standard deviation of the parameter.

Table 3: The $\sqrt{4 \widetilde{D} t}$ parameter used to evaluate the sharpness of the interface in the asdeposited case.

\begin{tabular}{|c|c|c|c|}
\hline Ti as-deposited & $\sqrt{4 \widetilde{D} t}$ & Y as-deposited & $\sqrt{4 \widetilde{D} t}$ \\
\hline $\mathrm{Fe}$ & $2.34 \pm 0.10$ & $\mathrm{Fe}$ & $1.04 \pm 0.06$ \\
\hline $\mathrm{Ti}$ & $2.32 \pm 0.04$ & $\mathrm{Y}$ & $1.24 \pm 0.14$ \\
\hline
\end{tabular}

As can be seen in Table 3, the substrate-thin film interface is sharper in the case of the asdeposited yttrium sample (lower value of $\widetilde{D}$ ). This is in agreement with the ADF and TEM images for the as-deposited samples: a thin intermixed region was found for the titanium asdeposited sample but not the yttrium as-deposited sample. This difference could arise due to systems' differences in the enthalpy of mixing with iron (Table 5), or the differing deposition velocities.

The application of collision cascade transport theory suggests the translational kinetic energy of the sputtered particles belongs to the Thompson distribution after normalization (84):

$$
\frac{d N}{d E_{t}} /_{N} \propto \frac{2 E_{b} E_{t}}{\left(E_{t}+E_{b}\right)^{3}}
$$

where $\frac{d N}{d E_{t}}$ is the number of particles within a differential energy interval, $E_{t}$ is the translational kinetic energy and $E_{b}$ is the surface binding energy of the sputtered atoms. This distribution has a very broad maximum probability centered at $1 / 2 E_{b}$. The surface binding energy values, as provided by the SRIM code are $4.24 \mathrm{eV} /$ atom for yttrium and $4.89 \mathrm{eV} /$ atom for titanium. Because titanium and yttrium were deposited using the same sputtering system and at the same gas pressure, it is expected that the energy of the titanium particles impinging against the substrate would be slightly higher in the case of the yttrium as-deposited sample. This, together with the higher chemical affinity between Fe-Ti as compared to Fe-Y could justify the different sharpness observed in the interfaces of the two materials. 


\subsection{Radiation and temperature effects}

The same fitting analysis that was performed for the as-deposited samples was repeated for the exposed and irradiated samples to determine how the diffusion coefficient parameter changes with the various thermal and irradiation conditions.

\subsubsection{Irradiation and exposure at $300^{\circ} \mathrm{C}$}

For both the Ti- and Y-coated samples exposed at $300^{\circ} \mathrm{C}$, the temperature was too low to promote marked diffusion of atoms given the low exposure time. After irradiation, some differences in sample behavior are observed.

In the titanium-coated sample, the interface between the substrate and the coating increased in length-scale as a result of interdiffusion of atoms. In Figure 5-3, the experimental concentration profile and the calculated diffusion profile for iron and titanium are shown Averaging of the 3 individual line scans generates the results found in Table 4.
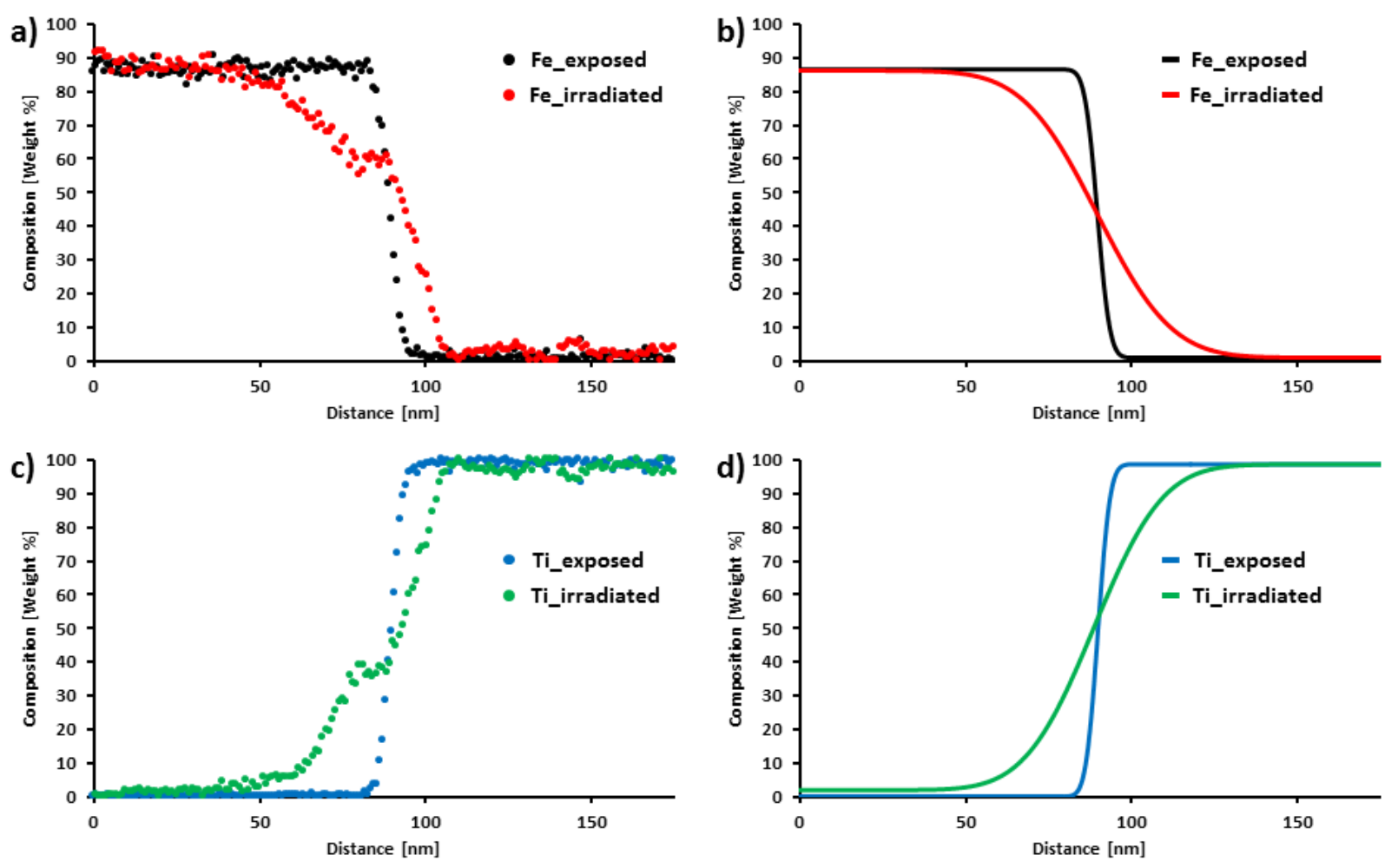

Figure 5-3: Diffusion profile of iron and titanium in the titanium-coated sample exposed and irradiated at $300 C^{\circ}$ : (a and c) experimental profiles (b and d) fitted profiles.

Comparing the two sample conditions reveals that including irradiation increases the interdiffusion coefficient by 2 orders of magnitude. This is likely due to radiation-induced point defects increasing the vacancy concentration significantly over that expected from thermal equilibrium at $300^{\circ} \mathrm{C}$, providing a considerable boost to the number of active diffusion sites, and thus increasing the effective interdiffusion coefficient. This hypothesis is supported by the observed composition profiles: in the substrate portions of the line scans, where the expected 
radiation damage profile (Figure 5-4) is higher than in the thin coating, the ingress of coating elements is enhanced relative to the egress of the substrate into the coating. Since the damage profile of the substrate is largely unaffected by the coating material, due to the thin nature of the coatings, it is not surprising that the irradiated samples' interdiffusion coefficients are similar in magnitude.

Table 4: Interdiffusion coefficients of iron and titanium $\left(D_{\mathrm{Fe} \text {-Ti }}\right)$ and iron and yttrium $\left(D_{\mathrm{Fe}-Y}\right)$ as calculated from the fitting parameter of the concentration profile for the samples exposed and irradiated at $300^{\circ} \mathrm{C}$.

\begin{tabular}{|c|c|c|c|}
\hline Ti_300_exposed & D $_{\mathrm{Fe}-T i}\left[\mathbf{c m}^{2} / \mathbf{s e c}\right]$ & Ti_300_irradiated & D $_{\mathrm{Fe}-\mathbf{Y}}\left[\mathbf{c m}^{2} / \mathbf{s e c}\right]$ \\
\hline $\mathrm{Fe}$ & $5.19 \times 10^{-18}$ & $\mathrm{Fe}$ & $1.58 \times 10^{-16}$ \\
$\mathrm{Ti}$ & $4.44 \times 10^{-18}$ & $\mathrm{Ti}$ & $1.22 \times 10^{-16}$ \\
\hline Y_300_exposed & $\mathbf{D}_{\mathrm{Fe}-\mathbf{Y}}\left[\mathbf{c m}^{2} / \mathbf{s e c}\right]$ & Y_300_irradiated & $\mathbf{D}_{\mathrm{Fe}-\mathbf{Y}}\left[\mathbf{c m}^{2} / \mathbf{s e c}\right]$ \\
\hline $\mathrm{Fe}$ & $3.89 \times 10^{-18}$ & $\mathrm{Fe}$ & $1.53 \times 10^{-16}$ \\
$\mathrm{Y}$ & $3.33 \times 10^{-18}$ & $\mathrm{Y}$ & $1.20 \times 10^{-16}$ \\
\hline
\end{tabular}

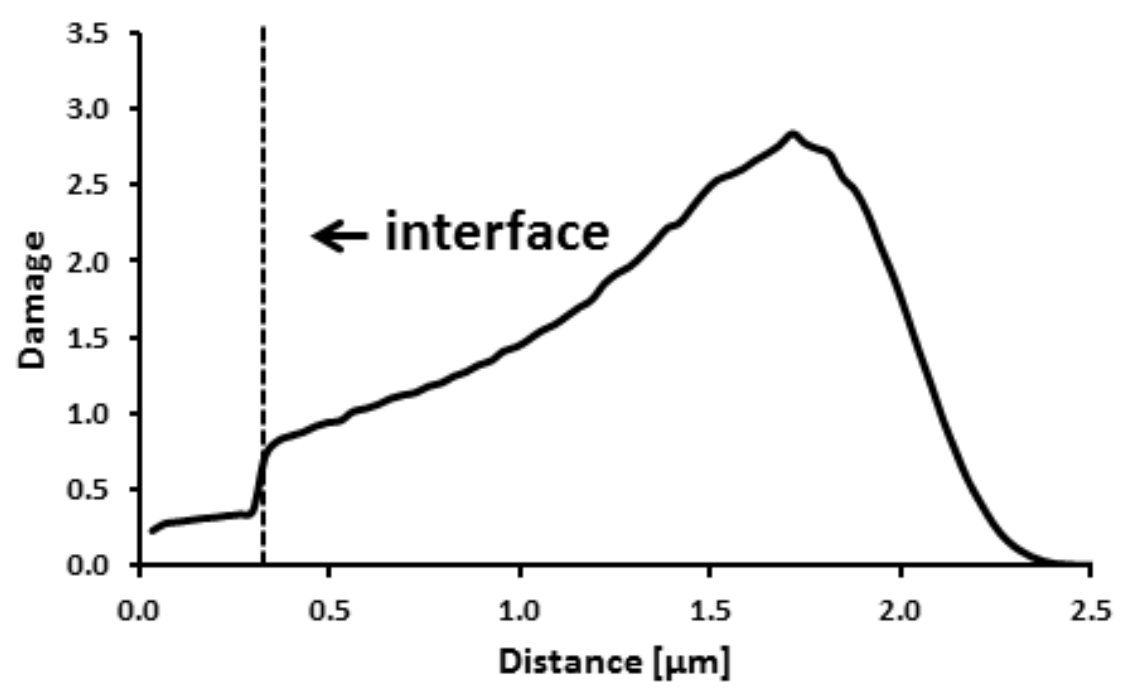

Figure 5-4: Damage profile for the titanium-coated sample irradiated with $5 \mathrm{MeV}$ nickel ions.

In the yttrium-coated sample, nanoparticles enriched in iron and depleted in yttrium were found in the coating. The absence of chromium in the EDS line scan profile precludes the presence of intermetallic compounds containing chromium, but the classification of the precipitates remains difficult: acquisition of an accurate diffraction pattern is complicated by the size of the nanoparticles, and due to the lack of extensive contrast with the matrix, HRTEM imaging is also difficult. Furthermore, the Fe-Y binary phase diagram (Figure 5-5) predicts four intermetallic compounds can form when iron and yttrium combine.

When determining the nature of a precipitate by its chemical composition, it must be noted that STEM images (and therefore also EDS chemical analysis) gives a 2-D projection of a 
system that is a 3-D feature. The experimentally determined composition will thus be influenced by the surrounding matrix. In order to determine the real composition of the particle it is necessary to know the thickness of the sample at that position, the thickness of the precipitate, and both the distant and local matrix composition. Precipitate thickness can be estimated by making assumptions regarding the geometry (e.g. the spherical radius $r$ in Figure 5-6 could be estimated by halving the length of the deviating composition found in a top-down line scan). Only if all these values are known the chemical composition of the particle can be determined exactly. The determination of the exact composition of these precipitates will be part of continuing work.

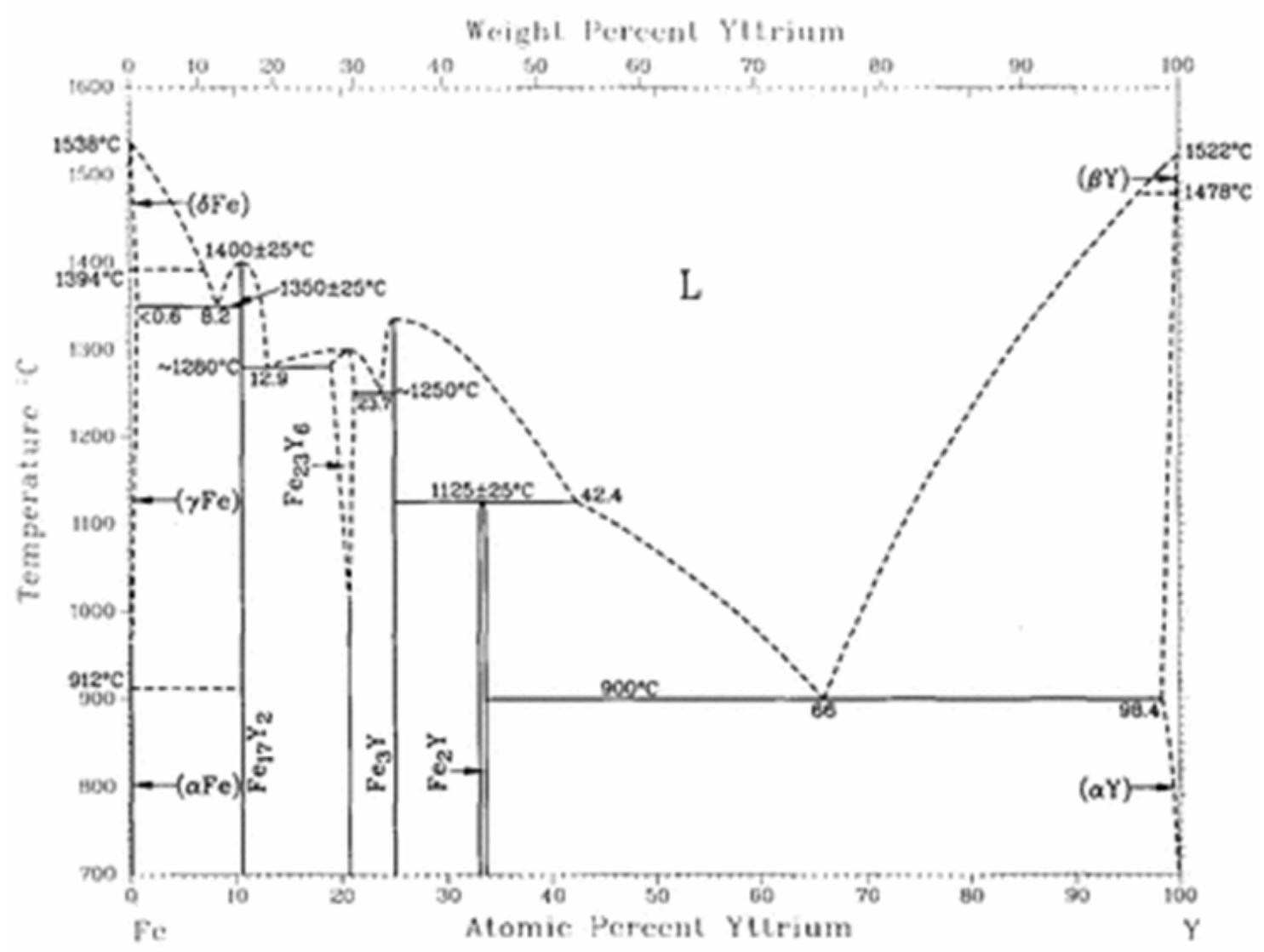

Figure 5-5: Binary phase diagram of the Fe-Y system (from Ref (96)).

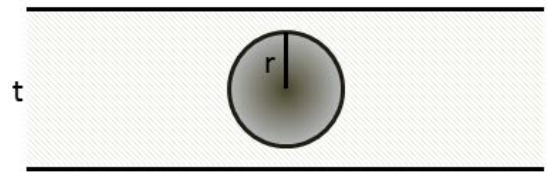

Figure 5-6: Spherical precipitate with radius r embedded in a matrix of thickness $t$. The acquisition of the nanoprecipitate's EDS spectrum will be influenced by the matrix.

When the titanium oxide sample was exposed at $300^{\circ} \mathrm{C}$ no major changes were observed in sample morphology. Even after irradiation the sharpness of the interface remained, though 
chromium-based nanoprecipitates were observed in the coating. As with the metallic coatings, the titanium oxide sample was very stable during exposure and also during irradiation, with no major modifications occurring at the substrate-thin film interface.

When the yttrium oxide-coated sample was exposed at the same temperature, a chromium-enriched layer was found between the substrate and the thin film. If the EDS line scan is evaluated in terms of the atomic fraction instead of the weight fraction, it seems that the nominal composition of the sample at the precipitate is similar to that of $\mathrm{Cr}_{2} \mathrm{O}_{3}$. Irradiation at $300^{\circ} \mathrm{C}$ suppresses the formation of this layer, instead promoting the formation of an evenly mixed, $75 \mathrm{~nm}$ wide region. Following this intermixed region, the coating is found. Some chromium-rich, iron-depleted nanoprecipitates found deep inside the substrate of the exposed sample were also found in the substrate of the irradiated sample. Inside the coating, some brightcontrast nanoparticles in the ADF images were determined to be enriched in iron and depleted in yttrium.

\subsubsection{Irradiation and exposure at $500^{\circ} \mathrm{C}$}

The titanium-coated, thermally exposed samples showed the formation of a wide (extending for hundreds of nanometers) intermixed region, with a stable, intermediate chemical composition. Irradiation further increases the extent of the intermixed region $150 \mathrm{~nm}$, increasing from $\sim 340 \mathrm{~nm}$ to $\sim 490 \mathrm{~nm}$. The Fe-Ti phase diagram (Figure 5-7) indicates two equilibrium intermetallics exist: FeTi and $\mathrm{Fe}_{2} \mathrm{Ti}$.

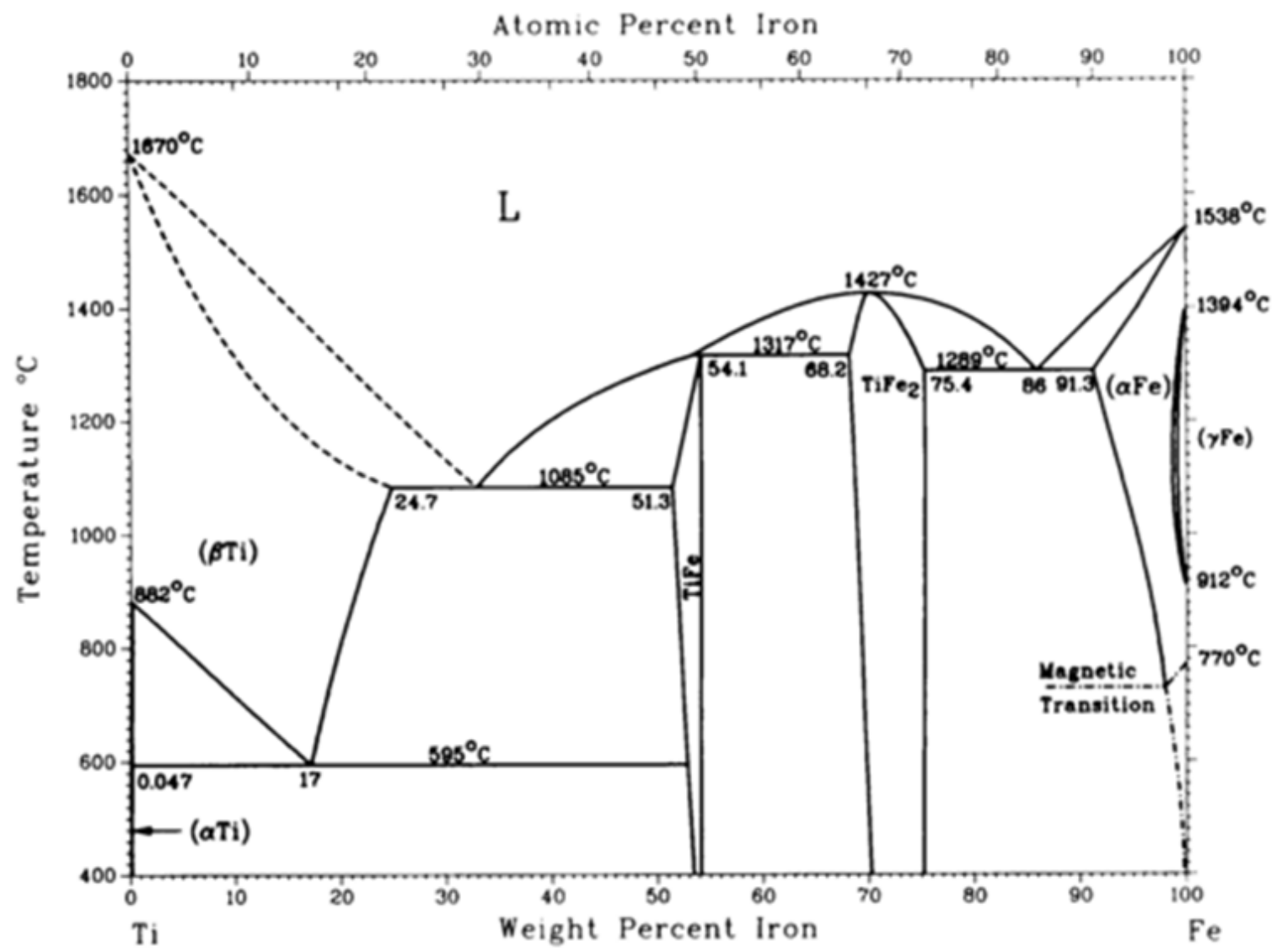

Figure 5-7: Binary phase diagram of the Fe-Ti system (from Ref. (65)). 
A detailed STEM-EDS and TEM analysis of the irradiated sample indicates the nanoparticles form the $\mathrm{Fe}_{2} \mathrm{Ti}$ phase. This phase is hexagonal, with lattice parameter of $\mathrm{a}=0.4787$ and $c=0.7815$. Figure $5-8$ shows TEM micrographs of this region from the irradiated and thermally exposed sample at $500^{\circ} \mathrm{C}$. The diffraction patterns acquired from the circled grains in Figure 5-8 are compatible with a hexagonal structure having the lattice parameter of $\mathrm{Fe}_{2} \mathrm{Ti}$.
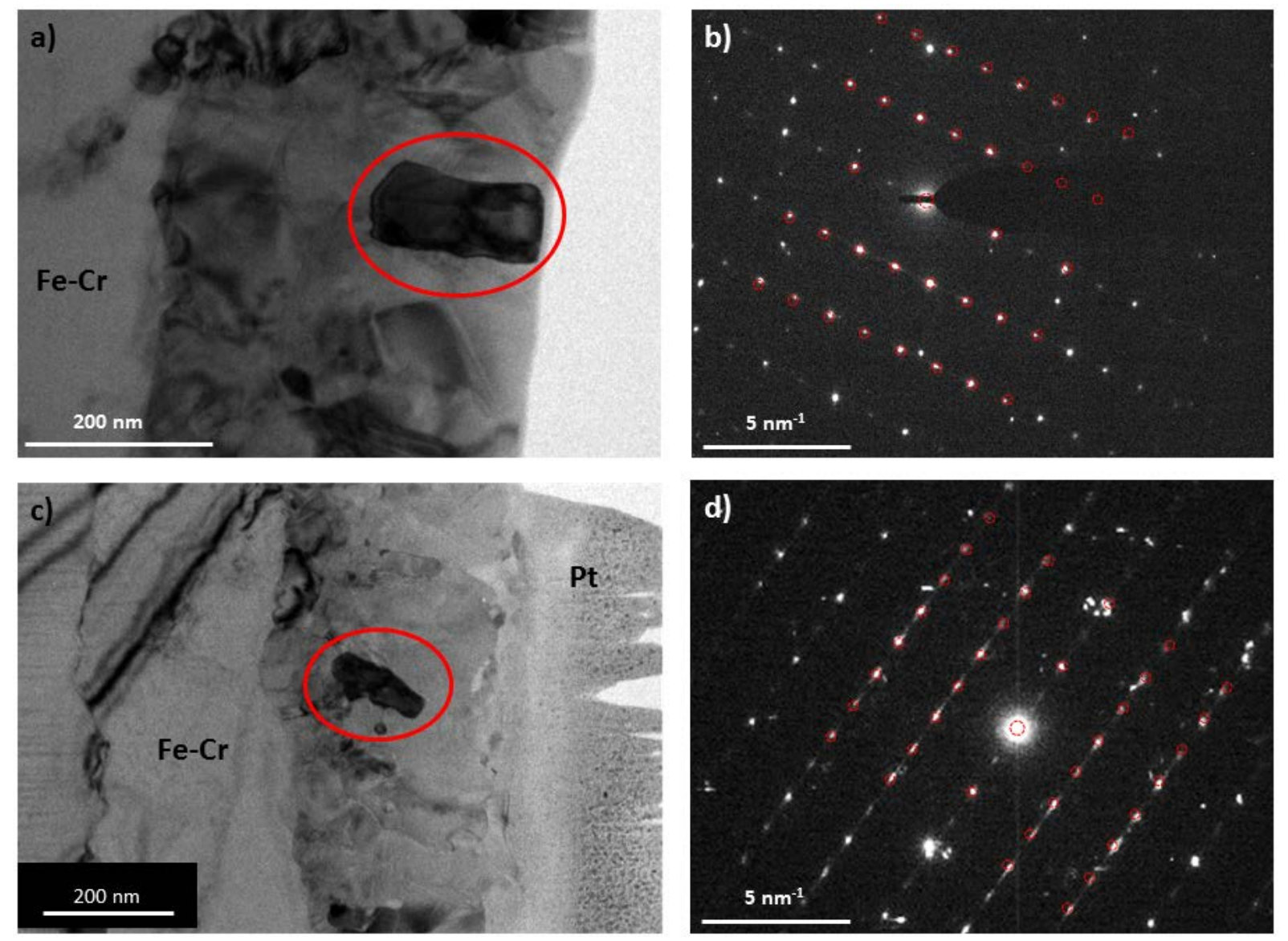

Figure 5-8: $\mathrm{Fe}_{2}$ Ti phase in the Ti coated sample a) irradiated at $500^{\circ} \mathrm{C}$ c) exposed at $\left.500^{\circ} \mathrm{C} . \mathrm{b}\right)$ and d) are the $S A D P$ acquired from the dark grain in the center of the image and the red circles superimposed are the simulated diffraction pattern for the [1010] hcp zone axis.

Finally, additional nanoparticles enriched in titanium and depleted in iron were found in the $\mathrm{Fe}_{2} \mathrm{Ti}$ layer, and, in some cases along grain boundaries, away from the original position of the coating. A detailed study of the chemical composition of these samples was performed by STEM-EDS, in order to try to reveal their nature. The relative enrichment of titanium is similar in both the samples, but again this has yet to be conclusively determined by accounting for the matrix thickness in the region of the precipitate. Considering that the local matrix is $\mathrm{Fe}_{2} \mathrm{Ti}$, it is speculated that these nanoprecipitates are FeTi intermetallics, the other stable intermetallic in the Fe-Ti system. This would be consistent with studies found in literature for thermal annealing (70) and irradiations $(74 ; 72)$ of Fe-Ti multilayers. 
In the yttrium-coated sample, exposure at $500^{\circ} \mathrm{C}$ caused the formation of a wide intermixed region with almost constant composition; irradiation caused the formation of regions predominately enriched in iron alternating with regions predominantly enriched in yttrium. These behaviors can be justified based on the enthalpy of mixing of the different couples of elements of the system. Calculated enthalpies of mixing (with Miedema's model (97)) for the couples of elements of interest in the system are shown in Table 5. The strongly negative calculated value of the enthalpy of mixing in the Fe-Ti couple can justify the ease with which these two elements form a solid solution in the exposed and irradiated case. For the Fe-Y couple, this driving force is considerably smaller.

Table 5: Enthalpy of mixing for different bilayers of interests for this work as calculated using the Miedema's model.

\begin{tabular}{|c|c|}
\hline Binary system & $\boldsymbol{\Delta H}_{\boldsymbol{m} \boldsymbol{i x}}(\boldsymbol{K J} / \boldsymbol{m o l})$ \\
\hline $\mathrm{Fe}-\mathrm{Ti}$ & $-25 \mathrm{KJ} / \mathrm{mol}$ \\
$\mathrm{Fe}-\mathrm{Y}$ & $-1 \mathrm{KJ} / \mathrm{mol}$ \\
$\mathrm{Cr}-\mathrm{Ti}$ & $-11 \mathrm{KJ} / \mathrm{mol}$ \\
$\mathrm{Fe}-\mathrm{Cr}$ & $-2 \mathrm{KJ} / \mathrm{mol}$ \\
$\mathrm{Cr}-\mathrm{Y}$ & $16 \mathrm{KJ} / \mathrm{mol}$ \\
\hline
\end{tabular}

The evolution of the ceramic-coated samples was again a little bit different. Exposure of the titanium oxide-coated sample at $500^{\circ} \mathrm{C}$ did not cause major modification to the morphology or the chemistry of the sample, unlike when combined with irradiation. The most striking effect of incorporating radiation was the sputtering of major parts of the coating. Additionally, the oxide-coated samples were more susceptible to chromium ingress. The yttrium oxide-coated sample followed a trend expected from observations of the $300^{\circ} \mathrm{C}$ sample. Thermal exposure forms Cr-rich inclusions on the substrate-thin film interface. These inclusions are compositionally similar to the ones found in the $300^{\circ} \mathrm{C}$ exposed sample, but are larger. Irradiation again suppresses the nanoprecipitate formation and promotes the formation of an extended, evenly intermixed region between the substrate and the coating.

\subsubsection{Irradiations and exposure at $700^{\circ} \mathrm{C}$}

The final irradiation experiment was performed at $700^{\circ} \mathrm{C}$. In the titanium-coated sample, almost no intermixing between the coating and the substrate was observed. The root cause of this observation is still under investigation.

The case of the yttrium-coated sample was different. As shown in Figure 4-17 there were very few distinguishing factors between the thermally exposed and the exposed+irradiated sample. Use of multiple EDS line scans determined the intermixed region was similar in length scales. Reasons for such a similarity between the exposed and the irradiated region of this sample could lie in the elevated temperature at which the irradiation was done. At very high temperature, the equilibrium point defect concentration in the sample is very high and their number could be unaffected by the ones produced during irradiation. Real ODS steels behave similarly, demonstrating resistance to segregation and intermixing under irradiation at high 
temperatures, some even lower than $700^{\circ} \mathrm{C}$ (49). In the yttrium-coated samples, very small nanoprecipitates were observed following the experiment. ADF images indicate that several show a darker contrast than the matrix material but are not chemically distinct. Other nanoprecipitates showed a brighter ADF contrast than the matrix, and EDS confirmed that they are enriched in yttrium and depleted in iron.

Figure 5-9 summarizes the intermixing regions of the metallic-coated samples after all the exposure and irradiation experiments. As expected from the thermodynamic enthalpy of mixing, the intermixing length is larger for the Fe-Ti samples than the $\mathrm{Fe}-\mathrm{Y}$ samples at comparable temperatures. When thermal effects are not dominant, irradiation with $5 \mathrm{MeV}$ nickel ions is shown to increase the length scale of the intermixed region.
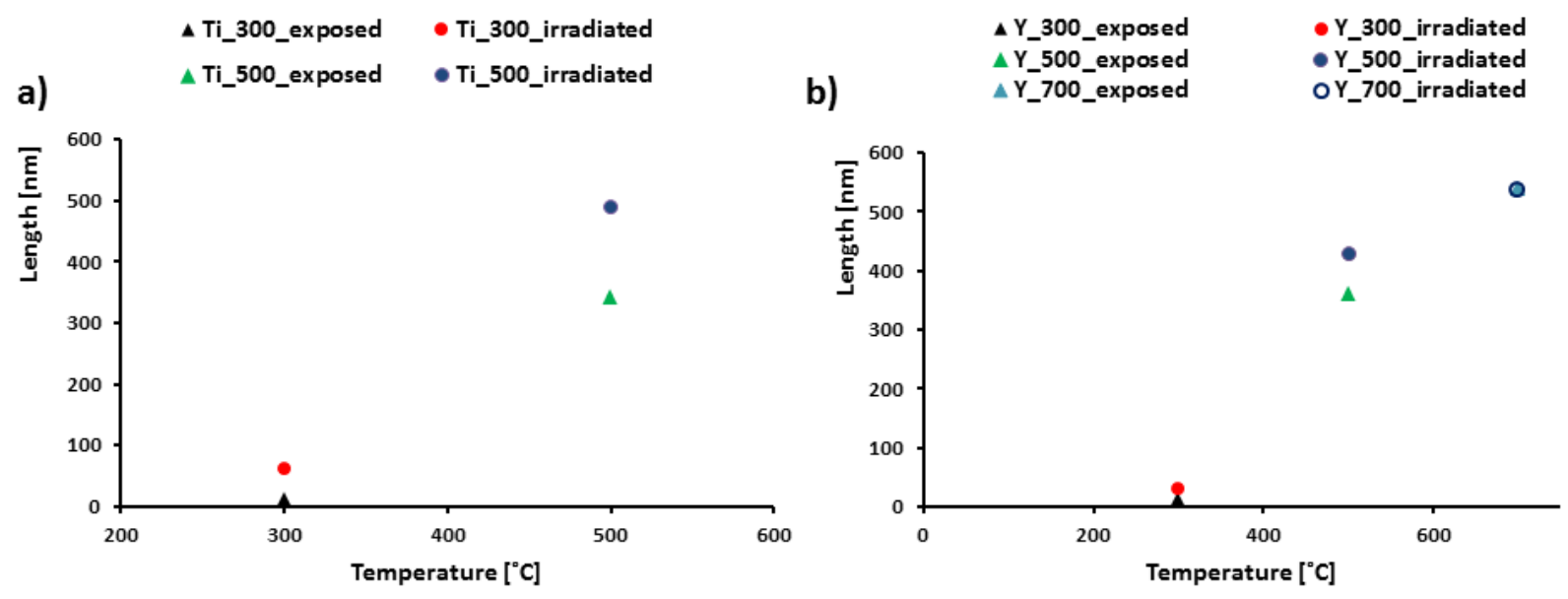

\section{Figure 5-9: Length-scale of interface mixing in function of temperature for the different samples exposed and irradiated at high temperature a) titanium b) yttrium.}

For the titanium oxide sample, the microstructure following the $700^{\circ} \mathrm{C}$ experiment closely resembles the microstructure observed after the $500^{\circ} \mathrm{C}$ experiment. At $700^{\circ} \mathrm{C}$, the experiment duration is sufficient to promote the diffusion of chromium into the coating. Unlike in the metallic-coated samples, the diffusion of iron into the oxides is suppressed. Irradiation modifies the chromium diffusion profile inside the coating, promoting the formation of small precipitates instead of the classical diffusion profile. The $700^{\circ} \mathrm{C}$ treated, yttrium oxide-coated sample also followed in the footsteps of the $500^{\circ} \mathrm{C}$ experiment: thermal exposure forms chromium oxides at the interface, and irradiation suppresses their formation in favor of a large intermixed region. 


\section{CHAPTER 6 Summary and Future Work}

\subsection{Summary}

In this work thus far the stability and the intermixing effects between Fe-12\%Cr binary alloy substrates and two different metallic coatings, titanium and yttrium have been evaluated using irradiation with $5 \mathrm{MeV}$ nickel ions at high temperatures of $300^{\circ} \mathrm{C}, 500^{\circ} \mathrm{C}$, and $700^{\circ} \mathrm{C}$. This system is of interest in the field of nuclear materials because the substrate and the coating simulate the matrix and precipitates of ODS steels which are very promising candidates as nextgeneration nuclear reactor materials. A small beam was used to irradiate the samples and therefore just a small fraction of their surface was effectively irradiated and the remaining part of it was exposed to the same temperature conditions, but not irradiation. TEM samples were prepared from both these regions and the results were analyzed and compared. In the asdeposited case, it was observed that the Fe-12\%Cr-yttrium interface was sharper than the Fe$12 \%$ Cr-titanium one, and it is speculated that this was caused by the greater energy with which titanium particles are impinging against the substrate during the deposition process combined with a greater chemical affinity between iron and titanium as compared to iron and yttrium.

In the lowest temperature irradiation, temperature effects did not cause major modifications to the system. The interface between the substrate and the coating does not appear changed in terms of the morphology or the chemistry. On the other hand, irradiation caused an increase in the extent of the interface, with inter-diffusion mainly between iron and the coating material. Inter-diffusion coefficients for the two systems were calculated for the exposed and the irradiated case and it was observed that irradiation caused an increase in the diffusion coefficient by two orders of magnitude as compared to the simple exposure. Also, it was observed that iron was more prone to diffuse into the coating as compared to chromium (in the substrate alloy), and this is due to the lower values of the enthalpy of mixing for the Fe-Ti and Fe-Y as compared to the $\mathrm{Cr}-\mathrm{Ti}$ and $\mathrm{Cr}-\mathrm{Y}$.

At $500^{\circ} \mathrm{C}$, major modifications occurred in the samples. For the titanium-coated sample, both exposure and irradiation caused the formation of a wide intermixed region, in which the composition, as revealed by EDS, was constant and intermediate between the substrate and the coating. Diffraction patterns acquired from these regions are consistent with that expected of $\mathrm{Fe}_{2} \mathrm{Ti}$, an intermetallic compound formed between iron and titanium which has a hexagonal structure. The additional effect of irradiation is that this intermixed region is considerably wider. Within it, small nanoprecipitates are observed. Based on similar systems studied in literature it was speculated that these particles are FeTi intermetallic precipitates. The exact determination of their composition and crystal structure will be a part of continuing work. A different behavior was observed in the yttrium-coated sample. Again, an intermixed region was observed after exposure and irradiation, with the irradiation increasing its extent. In the exposed sample, this region has a constant mixed composition, but when irradiated, an alternating composition profile is present, in which the dominant element is either iron or yttrium.

The last examined temperature was $700^{\circ} \mathrm{C}$. The titanium-coated sample showed an unexpected behavior and will be prepared again for confirmatory analysis. The yttrium-coated samples exhibited no clear difference between the exposed and irradiated samples. TEM analysis 
of the yttrium-coated samples irradiated and exposed at $500^{\circ} \mathrm{C}$ and $700^{\circ} \mathrm{C}$ will be also a part of this continuing work.

Oxide-coated samples were also examined, and they performed differently than the metallic-coated samples. Titanium oxide was found to create more stable interfaces under thermal and radiation exposure than yttrium oxide, though under irradiation the external surface was prone to sputter at $500^{\circ} \mathrm{C}$ and $700^{\circ} \mathrm{C}$. Despite formation of some Cr-rich nanoprecipitates, the substrate-titanium oxide interface is still sharp. In the yttria-coated samples, irradiation instead suppressed the formation of a chromium-enriched layer that would otherwise form at the interface.

\subsection{Future Work}

Many aspects of the stability of the interface between the chosen substrates and the different thin films deposited have been described and explained; yet others remain for further analysis and exploration:

1. Determine more definitively the composition of the precipitates that form as a result of ion irradiation and bench-mark this information against those that form in the thermal exposed samples (using further HRTEM and Energy Electron Loss Spectrum (EELS) analysis).

2. Study the effect of dose and the dose rate on the evolution phases in the system. These would be done by irradiations at $300^{\circ} \mathrm{C}$, at three different doses and three different doses rate using noble metal ions, such as gold or platinum. Figure 6-1 shows the difference in the damage caused to the sample between nickel $5 \mathrm{MeV}$ nickel and $2.7 \mathrm{MeV}$ gold ions. By employing heavy noble metal ions, the system can be subjected to very high doses in a short amount of time, by implanting the ions closer to the interface.

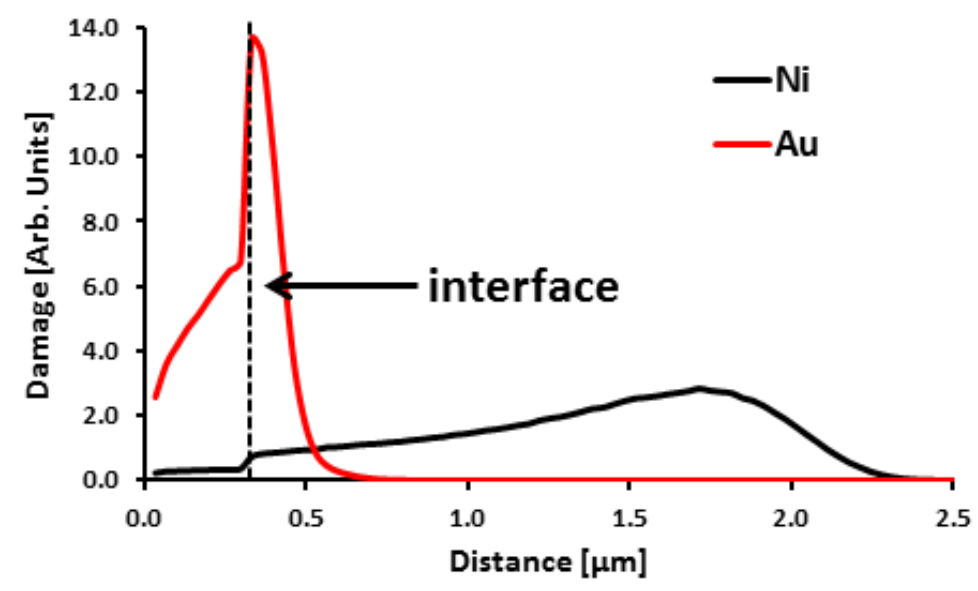

Figure 6-1: Damage profiles of $5 \mathrm{MeV}$ nickel ions and 2.7 MeV gold ions. 
3. Investigate neutron irradiated ODS samples and correlate the findings with thin film studies. Neutron irradiated 9-\%Cr ODS sample (irradiated at $500^{\circ} \mathrm{C}$ and $3 \mathrm{dpa}$ ) has already been prepared for this study.

4. Investigate the role of oxygen in the response to radiation of a pure bcc iron sample, employing the modeling framework developed during the project. 


\section{BIBLIOGRAPHY}

1. World Energy Needs and Nuclear Power. http://world-nuclear.org/info/Current-and-FutureGeneration/World-Energy-Needs-and-Nuclear-Power/\#.Uh0Z49LEOA8. [Online]

2. High-chromium ferritic and martensitic steels for nuclear applications. R. Klueh, D. Harries. s.l. : ASTM, 2001.

3. $\quad$ http://energy.gov/ne/articles/paving-path-next-generation-nuclear-energy. http://energy.gov/ne/articles/paving-path-next-generation-nuclear-energy. [Online]

4. J.J. Duderstadt, L.J. Hamilton. Nuclear Reactor Analysis. s.l. : John Wiley and Sons,, 1976.

5. Materials challenges for nuclear systems. Todd Allen, Jeremy Busby, Mitch Meyer, David Petti. 12, s.l. : Materials Today, 2010, Vol. 13, pp. 14-23.

6. The Stability of 9Cr-ODS Oxide Particles Under Heavy-Ion Irradiation. T.R. Allen, J. Gan, J.I. Cole, S. Ukai, S. Shutthanandan, S. Thevuthasan. s.l. : Nuclear Science and Engineering, 2005, Vol. 151, pp. 305-312.

7. Heavy-ion irradiation effects on the morphology of complex oxide particles in oxide dispersion strengthened ferritic steels. H. Kishimoto, K. Yutani, R. Kasada, 0. Hashitomi, A. Kimura. s.l. : Journal of Nuclear Materials, 2007, Vols. 367-370, pp. 179-184.

8. HRTEM study of oxide nanoparticles in K3-ODS ferritic steel developed for radiation tolerance. L. Hsiung, M. Fluss, S. Tumey, J. Kuntz, B. El-Dasher, M. Wall, B. Choi, A. Kimura, F. Willaime, Y. Serruys. 2, s.l. : Journal of Nuclear Materials, 2011, Vol. 409, pp. 72-79.

9. C.T. Calrebrough, L.M. Forwood. Electron Microscopy of Interfaces in Metals and Alloys. s.l. : Adam Hilger, 1991.

10. M.J. Demkowicz, J. Wang and R.G. Hoagland. Dislocation in Solids. s.l. : Elsevier, 2008.

11. EPR Observation of Close Frenkel Pairs in Irradiated ZnSe. G.D.Watkins. s.l. : Phys Rev lett., 1974, Vol. 33, pp. 223-225. 
12. G.S.Was. Fundamentals of Radiation Materials Science: Metals and Alloys. New York: Springler, 2007.

13. standards, ASTM. http://enterprise.astm.org/filtrexx40.cgi?+REDLINE_PAGES/E521.htm. Standard Practice for Neutron Radiation Damage Simulation by Charged-Particle Irradiation. [Online]

14. Fundamental aspects of nuclear reactor fuel elements: prepared for the Division of Reactor Development and Demonstration. Olander, D. [ed.] Office of Public Affairs Technical Information Center. s.l. : Energy Research and Development Administration, 1976, p. of Tid 26711 P1.

15. Radiation-Induced Effects on Microstructure. Zinkle, S.J. s.l. : Comprehensive Nuclear Materials, 2012, Vols. Volume 1: Basic Aspects of Radiation Effects in Solids/Basic Aspects of Multi-Scale Modeling, pp. 65-98.

16. PhD thesis. Field, K.G. 2012.

17. B. Raj, M. Vijayalakshmi. Comprehensive Nuclear Materials. Oxford : Elsevier, 2012. pp. 97-121.

18. Limits of composition achievable by ion implantation. Z.L. Liau, J.W. Mayer. s.l. : Journal of Vacuum Technology, 1978, Vol. 15, p. 1629.

19. Ion Induced Metastable Phases. D.A. Lilienfeld, L.S. Hung and J.W. Mayer. s.l. : Nuclear Instruments and Methods in Physics Research B, 1987, Vol. 19/20, pp. 1-7.

20. Ion beam mixing in metallic and semiconductor materials. M. Nastasi, J.W. Mayer. s.l. : Materials Science and Engineering, 1994, Vol. R12, pp. 1-52.

21. Ion beam mixing in amorphous silicon I. Experimental investigation. S. Matteson, B.M. Paine, M.G. Grimaldi, G. Mezey, M.-A. Nicolet. s.l. : Nuclear Instruments and Methods, 1981, Vol. 182/183, pp. 43-51.

22. J.F. Ziegler, J.P. Biersack and U. Littmark. The Stopping and Range of Ions in Solids. s.l. : Pergamon Press, 1985.

23. Principles of ion Mixing. S. Matteson, M.-A. Nicolet. s.l. : Ann. Rev. Mater. Sci., 1983, pp. 339-363.

24. Ion Beam Mixing of $\mathrm{Cu}$-Au and $\mathrm{Cu}$-W Sustems. H. Westendorp, Z.-L H. Wang and F.W. Saris. s.l. : Nuclear Instruments and Methods, 1982, Vol. 194.

25. Comparison of Ion Beam Miing at Room Temperature and 40 K. B.M. Paine, M.A. Nicolet,R.G. Newcombe, D.A. Thompson. s.l. : Nuclear instruments and methods, 1981, Vol. 182/183, pp. 115-119.

26. P.G. Shewmon. Diffusion in Solids. New York : McGraw-Hill, 1963. 
27. H.C. Pearson, G.L. Casey. Point defects in Solids. New York : s.n., 1975. Vol. 2.

28. The Depth Resolution of Sputter Profiling. H.H. Andersen. s.l. : Applied Physics, 1979, Vol. 18, pp. 131-140.

29. Theoretical Aspects of Atomic Mixing Ion Beams. Gras-Marti, P. Sigmund and A. s.l. : Nuclear Instruments and Methods, 1981, Vol. 182/183, pp. 24-41.

30. Amorphization of thin multilayer films by ion mixing and solid state reaction. M.V. Rossum, U. Shreter, W.L. Johnson, M.A. Nicolet,. s.l. : Materials Research Society Symposia Proceedings, 1984, Vol. 27, pp. 127-132.

31. R.A. Swalin. Thermodynamics of Solids. New York : Wiley-Interscience, 1972.

32. Ion-beam mixing in pure and in immiscible copper bilayer systems. R. S. Averback, D. Peak, L. J. Thompson. s.l. : Applied Physics A, 1986, Vol. 39, pp. 59-64.

33. Influence of chemical driving forces in ion mixing of metallic bilayers. Y.T. Cheng, M.V. Rossum, M.A. Nicolet, and W.L. Johnson. s.l. : Appl. Phys. Lett., 1984, Vol. 45, p. 185.

34. When is thermodynamics relevant to ion-induced atomic rearrangements in metals? W.L. Johnson, Y.T. Chen, M.V. Rossum, M.A. Nicolet. s.l. : Nuclear Instruments and Metho\& in Physics Research B, 1985, Vol. 7/8, pp. 657-665.

35. Measurement of Equilibrium Concentrations of Vacancies in Copper. R.O. Simmons, R.W. Balluffi. s.l. : Phys. Rev., 1963, Vol. 129, pp. 1533-1544.

36. Ion-beam mixing with chemical guidance IV. Thermodynamic effects without invoking thermal spikes. A. Miotello, R. Kelly. [ed.] 275-288. s.l. : Surface Science, 1994, Vol. 314.

37. S. Matteson, J. Roth, M. Nicolet. s.l. : Rad Eff, 1979, Vol. 42, pp. 217-226.

38. Correlation between the cohesive energy and the onset of radiation enhanced diffusion in ion mixing. Y.T. Cheng, X.A. Zhao, T. Banwell, T. W. Workman, MA. Nicolet, W.L. Johnson. s.l. : J. Appl. Phys., 1986, Vol. 60, p. 2615.

39. Comparison of swelling and irradiation creep behavior of fcc-austenitic and bccferritic/martensitic alloys at high neutron exposure. F.A. Garner, M.B. Toloczko, B.H. Sencer. s.l. : J. Nucl. Mater., 2000, Vol. 276, pp. 123-142.

40. Improvement of Creep Strength of 9CrODS Martensitic Steel by Controlling Excess Oxygen and Titanium Concentrations. Satoshi Ohtsuka, Shigeharu Ukai, Masayuki Fujiwara, Takeji Kaito, Takeshi Narita. s.l. : Materials Transactions, Vol. 46, pp. 487-492. 
41. Creep constitutive equation of dual phase 9Cr-ODS steel. H. Sakasegawa, S. Ukai, M. Tamura, S. Ohtsuka, H. Tanigawa, H. Ogiwara, A. Kohyama, M. Fujiwara. s.l. : J. Nucl. Mater., 2008, Vol. 373, pp. 82-89.

42. Characterization of High Temperature Creep Properties in Recrystallized 12Cr-ODS Ferritic Steel Claddings. S. Ukai, T. Okuda, M. Fujiwara, T. Kobayashi, S. Mizuta, H. Nakashima. s.l. : J. Nucl. Sci. Technol., 2002, Vol. 39, pp. 872-879.

43. D.T. Hoelzer, J. Bentley, M.A. Sokolov, M.K. Miller, G.R. Odette, M.J. Alinger. s.l. : J. Nucl. Mater., 2007, Vols. 367-370 , pp. 166-172.

44. The transport and fate of helium in nanostructured ferritic alloys at fusion relevant He/dpa ratios and dpa rates. T. Yamamoto, G.R. Odette, P. Miao, D.T. Hoelzer, J. Bentley, N. Hashimoto, H. Tanigawa, R.J. Kurtz. s.l. : Journal of Nuclear Materials, 2007, Vols. 367-370, pp. 399-410.

45. Radiation response of a 9 chromium oxide dispersion strengthened steel to heavy ion irradiation. T.R. Allen, J. Gan, J.I. Cole, M.K. Miller, J.T. Busby. s.l. : Journal of Nuclear Materials , 2008, Vol. 375, pp. 26-37.

46. Nano-oxide particle stability of 9-12Cr grain morphology modified ODS steels under neutron irradiation. S. Yamashita, N. Akasaka, S. Ohnuki. s.l. : Journal of Nuclear Materials, 2004, Vols. 329-333, pp. 377-381.

47. Structure of Nano-Size Oxides in ODS Steels and Its Stability under Electron Irradiation. K. Oka, S. Ohnuki, S. Yamashita, N. Akasaka, S. Ohtsuka, H. Tanigawa. 10, s.l. : Materials Transactions, 2007, Vol. 48, pp. 2563-2566.

48. Effect of hydrogen ion/electron dual-beam irradiation on microstructural damage of a $12 \mathrm{Cr}$ ODS ferrite steel. Yang Zhanbing, Hu Benfu, H. Kinoshita, H. Takahashi, S. Watanabe. 1-3, s.l. : Journal of Nuclear Materials, 2010, Vol. 398, pp. 81-86.

49. Radiation stability of nanoclusters in nano-structured oxide dispersion strengthened (ODS) steels. A. Certain, S. Kuchibhatl, V. Shutthanandan, D.T. Hoelzer, T.R. Allen. s.l. : Journal of Nuclear Materials, 2013, Vol. 434, pp. 311-321.

50. The Theory of Phase Stability Under Irradiation. K.C. Russell. s.l. : Journal of Nuclear Materials, 1979, Vol. 83, pp. 176-185.

51. Structural Stability During Irradiation. J.A. Hudson. s.l. : J. Brit. Nucl. Energy Soc., 1975, Vol. 14, pp. 127-136.

52. Impact of irradiation on the microstructure of nanocrystalline materials. N. Nita, R. Schaeublin, M. Victoria. s.l. : Journal of Nuclear Materials, 2004, Vols. 329-333, pp. 953-957. 
53. Accumulation and recovery of defects in ion-irradiated nanocrystalline gold. Y. Chimi, A. Iwase, N. Ishikawa, M. Kobiyama, T. Inami, S. Okuda. s.l. : Journal of Nuclear Materials, 2001, Vol. 297, pp. 355-357.

54. Instability of irradiation induced defects in nanostructured materials. M. Rose, A.G. Balogh, H. Hahn. s.l. : Nuclear Instruments and Methods in Physics Research Section B: Beam Interactions with Materials and Atoms, 1997, Vols. 127-128, pp. 119-122.

55. Enhanced radiation tolerance of ultrafine grained Fe-Cr-Ni alloy. C. Sun, K.Y. Yu, J.H. Lee, Y. Liu, H. Wang, L. Shao, S.A. Maloy, K.T. Hartwig, X. Zhang. s.l. : Journal of Nuclear Materials, 2012, Vol. 420, pp. 235-240.

56. Efficient Annealing of Radiation Damage Near Grain Boundaries via Interstitial Emission. X.-M. Bai, A.F. Voter, R.G. Hoagland, M. Nastasi, B.P. Uberuaga. s.l. : Science, 2010, Vol. 327, pp. 1631-1634 .

57. Nanostructured $\mathrm{Cu} / \mathrm{Nb}$ multilayers subjected to helium ion-irradiation. $\mathbf{X}$. Zhang, Nan Li, O. Anderoglu, H. Wang, J.G. Swadener, T. Höchbauer, A. Misra, R.G. Hoagland. s.l. : Nuclear Instruments and Methods in Physics Research Section B: Beam Interactions with Materials and Atoms, 2007, Vol. 261, pp. 1129-1132.

58. The radiation damage tolerance of ultra-high strength nanolayered composites. A. Misra, M.J. Demkowicz, X. Zhang, R.G. Holand. s.l. : JOM, 2007, Vol. 59, pp. 62-65.

59. Ion-beam induced transformations in nanoscale multilayers: Evolution of clusters with preferred length scales. S. Bera, B. Satpati, D.K. Goswami, K. Bhattacharjee, P.V. Satyam, B.N. Dev. s.l. : Journal of Applied Physics, 2006, Vol. 99, p. 074301.

60. Interface enabled defects reduction in helium ion irradiated $\mathrm{Cu} / \mathrm{V}$ nanolayers. E.G. Fu, A. Misra, H. Wang, L. Shao, X. Zhang. s.l. : Journal of Nuclear Materials, 2010, Vol. 407, pp. 178-188.

61. The influence of interfaces on the formation of bubbles in He-ion-irradiated Cu/Mo nanolayers. N. Li, J.J. Carter, A. Misra, L. Shao, H. Wang, X. Zhang. s.l. : Philosophical Magazine Letters, 2011, Vol. 91, pp. 19-29.

62. Interface Structure and Radiation Damage Resistance in $\mathrm{Cu}-\mathrm{Nb}$ Multilayer Nanocomposites. M.J. Demkowicz, R.G. Hoagland, J.P. Hirth. s.l. : Physical Review Letters, 2008, Vol. 100, p. 136102.

63. He ion irradiation damage in Fe/W nanolayer films. N. Li, E.G. Fu, H. Wangb, J.J. Carter, L. Shao, S.A. Maloy, A. Misra, X. Zhang. s.l. : Journal of Nuclear Materials, 2009, Vol. 389, pp. 233-238.

64. He ion irradiation damage in $\mathrm{Al} / \mathrm{Nb}$ multilayers. Nan Li, M. S. Martin, O. Anderoglu, A. Misra, L. Shao, H. Wang, X. Zhang. s.l. : Journal of Applied Physics, 2009, Vol. 105, p. 123522. 
65. T. B. Massalski, J. L. Murray, L. H. Bennett, H. Baker. Binary Alloy Phase Diagrams. Metals Park, OH : American Society for Metalls, 1986.

66. Phase formation sequence for the reaction of multilayer thin films of Nb/Al. K. Barmak, K. R. Coffey, D. A. Rudman, S. Foner. s.l. : J. Appl. Phys., 1990, Vol. 67, p. 7313.

67. Thin film reaction kinetics of niobium/aluminum multilayers. K. R. Coffey, K. Barmak, D. A. Rudman, S. Foner. s.l. : J. Appl. Phys. , 1992, Vol. 72, p. 1341 .

68. 20K. Barmak, C. Michaelsen, S. Vivekanand, F. Ma. s.l. : Philos. Mag, 1998, Vol. A 67, p. 167.

69. Microstructural evolution of Fe/Ti multilayers submitted to in situ thermal annealing. M.K. Lei, Z.L. Wu, T. Chen, B.S. Cao. s.l. : Thin Solid Films, 2006, Vol. 500, pp. 174179.

70. Solid State reaction of Fe/Ti nanometer-scale multilayers. T. Chen, Z.L. Wu, B.S. Cao, J. Gao, M.K. Lei. s.l. : Surface \& Coatings Technology, 2007, Vol. 201, pp. 50595062.

71. Structural change of hte Fe/Ti multilayer during annealing in the vacuum and hydrogen atmosphere. K. Tanaka, M. Otsuka. s.l. : International Journal of Hydrogen Energy, 1999, Vol. 24, pp. 891-898.

72. Structural transformations induced in Fe/Ti multilayers by Ar- and $\mathrm{Kr}$-ion irradiation. $\mathbf{M}$. Kopcewicz, J. Jagielski, T. Stobiecki. s.l. : J. Phys.: Condens. Matter, 2005, Vol. 17, pp. 2149-2165.

73. Microstructure of ion-bombarded $\mathrm{Fe}-\mathrm{Ti}$ and $\mathrm{Fe}-\mathrm{Ti}-\mathrm{C}$ multilayered films. J.P. Hirvonen, $\mathrm{M}$. Nastasi, J.W. Mayer. s.l. : J. Appl. Phys. 60, 1986, Vol. 3, pp. 980-984.

74. Fe-Ti metastable-phase formation by ion-beam mixing. R. Brennier, T. Capra, P. Thevenard, A. Perez, M. Treilleux, J. Rivory, J. Dupuy, G. Giraud. s.l. : Phys. Rev. B, Vol. 41, pp. 11784-11789.

75. Microstructural evolution, atomic migration, and FePt nanoparticle formation in ionirradiated $\mathrm{Pt}(\mathrm{Fe}) / \mathrm{C}(\mathrm{Fe})$ multilayers. S. Bera, S. Roy, K. Bhattacharjee, G. Kuri, B.N. Dev. s.l. : J. Appl. Phys., 2007, Vol. 102, p. 014308.

76. Nonmagnetic to magnetic nanostructures via ion irradiation. B.N. Dev, S. Bera, B. Satpati, D.K. Goswami, K. Bhattacharjee, P.V. Satyam, K. Yamashita, O.M. Liedke, K. Potzger, J. Fassbender, F. Eichhorn, R. Groetzschel,. s.l. : Microelectronic Engineering, 2006, Vol. 83, pp. 1721-1725.

77. J.J. Burton, R.S. Polizzotti. s.l. : Surface Science, 1977, Vol. 66, p. 1. 
78. Surface Metallurgy With High Energy Heavy Ion Beams. M.A.Z. Vasconcellos, J.A.T.

Borges Da Costa, W.H. Schreiner, I.J.R. Baumvol. s.l. : Nuclear Instruments and Methods in Physics Research, 1989, Vol. B37/38, pp. 653-660.

79. Formation, Dynamics, and Characterization of Nanostructures by Ion Beam Irradiation. Dhara, S. s.l. : Critical Reviews in Solid State and Materials Sciences, 2007, Vol. 32, pp. 1-52.

80. Interfaces under ion irradiation: growth and taming of nanostructures. K.H. Heinig, T. Müller, B. Schmidt, M. Strobel, W. Möller. s.l. : Appl. Phys. A, 2003, Vol. 77, pp. $17-25$.

81. Ion irradiation for controlling composition and structure of metal alloy nanoclusters in SiO2. V. Bello, G. De Marchi, C. Maurizio, G. Mattei, P. Mazzoldi, M. Parolin, C. Sada. s.l. : Journal of Non-Crystalline Solids, 2004, Vols. 345-346, pp. 685-688.

82. Creation of Self-Organized Nanostructures by KeV Ion Beam Irradiation. U.B. Singh, D.C. Agarwal, S.A. Khan, A. Tripathi, A. Kumar, R.K. Choudhury, B.K. Panigrahi, D.K. Avasthi. s.l. : Radiation Effect \& Defects in Solids, 2011, Vol. 166, pp. 553557.

83. S. Reiss, K.H. Heinig. s.l. : Nucl. Instr. Meth., 1994, Vol. B 84, p. 229.

84. D.L. Smith. Thin-Film Deposition. s.l. : McGraw-Hill, 1995.

85. Crystal Film Thickness Monitor. http://www.tungsten.com/crystal\%20film\%20thickness.pdf. [Online] Midwest Tungsten Service.

86. http://www.tangidyne.us/qcmmonitorcrystals.html. http://www.tangidyne.us/qcmmonitorcrystals.html. [Online]

87. NEC. Negative Iob Beam Sources. http://www.pelletron.com/negion.htm. [Online]

88. D.B. Williams, C.B. Carter. Transmission Electron Microscopy. 2009.

89. R.J. Keyse, A.G. Garratt-Reed, P.J. Goodhew, G.W. Lorimer. Introduction to Scanning Electron Microscopy. s.l. : Springer, 1998.

90. VMD - Visual Molecular Dynamics. Humphrey, W., Dalke, A. and Schulten, K. 1996, J. Molec. Graphics, Vol. 14, pp. 33-38.

91. Fast Parallel Algorithms for Short-Range Molecular Dynamics. Plimpton, S. 1995, J Comp Phys, Vol. 117, pp. 1-19.

92. Efficient implementation of the concentration-dependent embedded atom method for molecular-dynamics and Monte-Carlo simulations. Stukowski, Sadigh, Erhart, Caro. 7, s.l. : IOP Publishing, 10 1, 2009, Modeling and Simulation in Materials Science \& Engineering, Vol. 17, p. 075005. 
93. Stoller, R. E. Radiation Damage: Mechanisms and Modeling. [Presentation] Knoxville, TN, U.S.A. : Oak Ridge National Laboratory, June 2012. Joint EFRC Summer School.

94. Development of a Reactive Force Field for Iron Oxyhydroxide Systems. Aryanpour, M., van Duin, A. C. T. and Kubicki, J. D. 21, 2010, J. Phys. Chem. A, Vol. 114, pp. 62986307.

95. Parallel reactive molecular dynamics: Numerical methods and algorithmic techniques. $\mathbf{H}$. M. Aktulga, J. C. Fogarty, S. A. Pandit, A. Y. Grama. 2012, Parallel Computing, Vol. 38, pp. 245-259.

96. The Fe-Y (iron-yttrium) system. W. Zhang, G. Liu, K. Han. 3, s.l. : Journal of Phase Equilibria, 1992, Vol. 13, pp. 304-308.

97. Cohesion in Metals. A.R. Miedema, A.K. Niessen, F.R. de Boer, R. Boom, W.C.M. Matten. 1, s.l. : Physica B+C, 1980, Vol. 100, pp. 1-28.

98. Ion beam mixing in amorphous silicon II. Theoretical interpretation. S. Matteson, B.M. Paine and M-A. Nicolet. s.l. : Nuclear Instruments and Methods. Vol. 182/183, pp. 53-61.

99. Winterbon, K.B. Ion Implantation Range and Energy Deposition Distributions. New York : s.n., 1975. Vol. 2.

100. Studies of a Phenomenological Model of Ion Mixing. Y.T.Cheng, T. W. Workman, M.A. Nicolet, W. L. Johnson. s.l. : MRS Proceedings, 1986, Vols. 74-A.

101. Formation, Dynamics, and Characterization of Nanostructures by Ion Beam Irradiation. Dhara, S. s.l. : Critical Reviews in Solid State and Materials Sciences, 2007, Vol. 32, pp. 1-50.

102. Design of Radiation Tolerant Nanostructured Metallic Multilayers. X. Zhang, E. G. Fu, Nan Li, A. Misra, Y.-Q. Wang, L. Shao, H. Wang. s.l. : Transactions of the ASME, 2012, Vol. 134, pp. 041010-1.

103. ASM Handbook Volume 3.

104. Does the valence state of an ion affect its diffusivity? Part I: Oxygen activity dependence of the diffusion of iron in alumina-doped MgO. E. Chen, T.L. Tsai, R. Dieckmann. 6, s.l. : Solid State Sciences, 2008, Vol. 10, pp. 735-745.

105. S.H. Moll, R.E. Ogilvie. s.l. : Trans. AIME, 1959, Vol. 215, p. 613.

106. Does the valence state of an ion affect its diffusivity? Part I: Oxygen activity dependence of the diffusion of iron in alumina-doped MgO. E. Chen, T.-L. Tsai, R. Dieckmann. s.l. : Solid State Science, 2008, Vol. 10, pp. 735-745. 
107. Atomic Radii in Crystals. Slater, J.C. s.l. : Journal of Chemical Physics, 1964, Vol. 41, pp. 3199-3205. 